
(ent. (2).

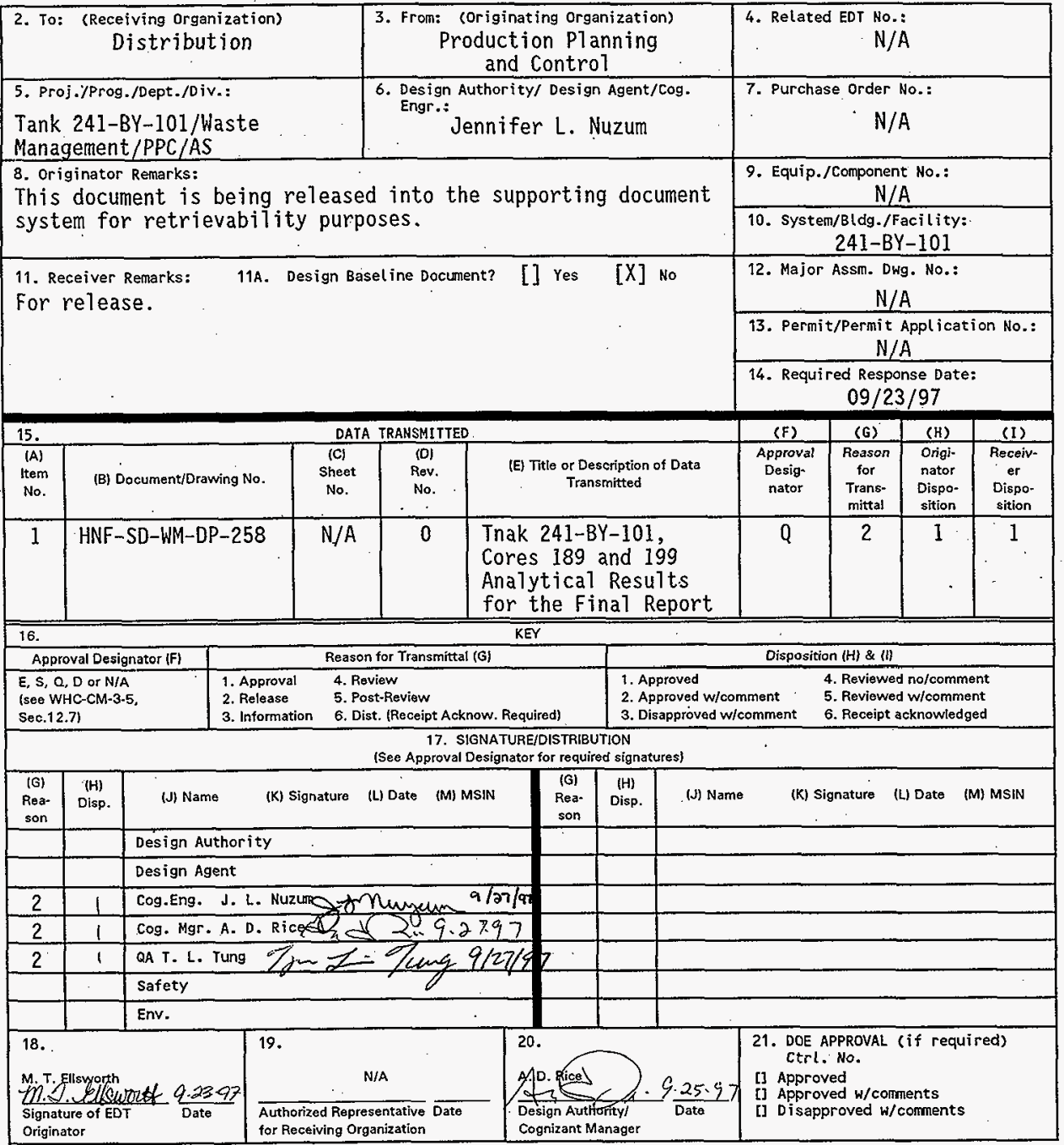




\title{
Tank 241-BY-101, Cores 189 and 199 Analytical Results for the Final Report
}

\author{
Jennifer L. Nuzum \\ Waste Management of Hanford, Inc., Richland, WA 99352 \\ U.S. Department of Energy Contract DE-AC06-96RL13200
}

$\begin{array}{lll}\text { EDT/ECN: } & \text { EDT }-620423 & \text { UC: } 2070 \\ \text { Org Code: } & 7576 B & \text { Charge Code: MD378 } \\ \text { B\&R Code: } & \text { EW } 3120074 & \text { Total Pages: } 177\end{array}$

Key Words: Tank 241-BY-101, 241-BY-101, BY-101, Core 189, Core 199, Analytical Resuits, Final Report

Abstract: $N / A$

TRADEMARK DISCLAIMER. Reference herein to any specific comercial product, process, or service by trade name, trademark, manufacturer, or otherwise, does not necessarily constitute or imply its endorsement, recommendation, or favoring by the United States Government or any agency thereof or its contractors or subcentractors.

Printed in the United States of America. To obtain copies of this document, contact: Document control Services, P.O. Box 950, Mailstop H6-08, Richland WA 99352, Phone (509) 372-2420; Fax (509) 376-4989.
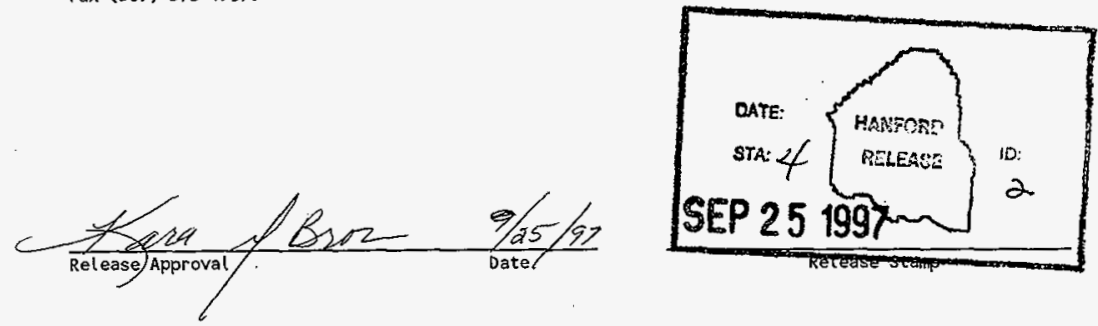

Approved for Public Release 
HNF-SD-WM-DP-258, REV. 0

\author{
ANALYTICAL SERVICES
}

\title{
TANK 241-BY-101, CORES 189 AND 199 ANALYTICAL RESULTS FOR THE FINAL REPORT
}

Project Coordinator: JENNIFER L. NUZUM

Prepared for the U.S. Department of Energy

Office of Environmental Restoration and Waste Management

$$
\text { by }
$$

222-S Laboratory

Waste Management of Hanford, Inc.

P.0. Box 700

Richl and, Washington 
HNF-SD-HM-DP-258, REV. 0

THIS PAGE INTENTIONALLY LEFT BLANK 
HNF-SD-HM-DP-258, REV.O

TABLE OF CONTENTS

Narrative . . . . . . . . . . . . . . . . . . 1

BY-101 Sample Breakdown (Attachment 1) ............ 11

Sample Data Summary ................... . . 17

Chain of Custody forms .................... 23

Photographs ....................... 32

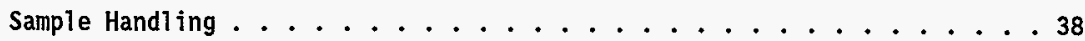

Extrusion Workl ist \# $18452(1239) \ldots . . . . . . . . . .40$

Extrusion Workl ist \# $18454(1236) \ldots . . . . . . . . . .41$

Extrusion Workl ist \# 18546 (1237) . . . . . . . . . . 42

Extrusion Workl ist \# $18573(1335) \ldots \ldots . \ldots . \ldots 43$

Sample Preparations . . . . . . . . . . . . . . . 44

Fusion Digestion Workl ist \# $18696(1358,1363) \ldots . . \ldots 46$

Water Digestion Workl ist \# $18697(1360,1364) \ldots . . . . . . .47$

BY-101 Bulk Density Worksheets . . . . . . . . . . . 48

Inorganic Analysis .................... 52

Differential Scanning Calorimetry (DSC)

DSC Worklist \# $19193(1362) \ldots \ldots \ldots \ldots$. . . . . . . . 54

DSC Workl ist \# $19383(1365) \ldots \ldots \ldots 59$

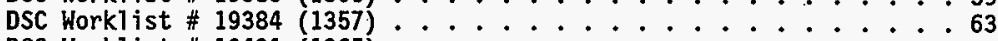

DSC Worklist \# 19431 (1365) $\ldots \ldots \ldots 67$

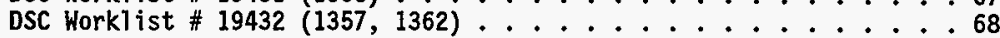

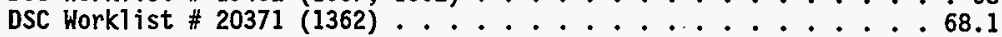

Thermogravimetric Analysis (TGA)

TGA Workl ist \# 18781 (1362) ................ . 69

TGA Worklist \# 19381 (1365) . . . . . . . . . . . . 75

TGA Worklist \# $19382(1357) \ldots \ldots \ldots . \ldots . \ldots 79$

Specific Gravity Analysis (SpG)

SpG WorkT ist \# $19389(1365) \ldots \ldots . \ldots . \ldots . \ldots 83$

Ion Chromatographic Analysis (IC)

IC Workl ist \# 18654 (1241) . . . . . . . . . . . . . . 87

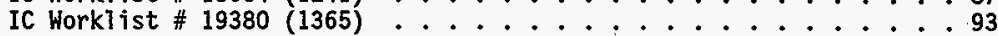

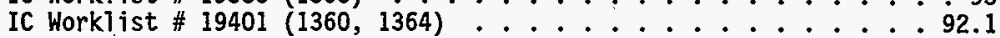


HNF-SD-WM-DP-258, REV. 0

TABLE OF CONTENTS (Continued)

Inductively Coupled Plasma Spectroscopy. Analysis (ICP)

ICP Workl ist \# 18729 (1241) . . . . . . . . . . . . . . 99

ICP Workl ist \# $19395(1365) \ldots \ldots . . \ldots \ldots$

ICP Work list \# $19462(1358,1363) \ldots \ldots \ldots \ldots$

Radiochemical Analysis ................ 135.1

Alpha Total Analysis (AT)

AT Worklist \# $19397(1365) \ldots \ldots \ldots$. . . . . . . . . . 136

AT Worklist \# $19405(1358,1363) \ldots \ldots \ldots \ldots . . . \ldots 143$

Opportunistic Analysis (Appendix A) ............. 152

This document consists of pages 1 through 157. Pages $i i, 2,12,18,24,33$, $39,49,53,58$ and 135.2 were intentionally left blank.

Also includes pages 135.1 and 135.2 . TRADEMARKS:

Perkin-Elmer is a Registered Trademark of Research and Manufacturing Company, Inc. Mettler is a Registered Trademark of Mettler Electronics. 
HNF-SD-WM-DP-258, REV , 0

NARRATIVE 
HNF-SD-WM-DP-258, REV . 0

THIS PAGE INTENTIONALLY LEFT BLANK 
HNF-SD-WM-DP-258, REV. 0

222-S ANALYTICAL SERVICES

\author{
TANK 241-BY-101, CORES 189 AND 199, \\ ANALYTICAL RESULTS FOR THE FINAL REPORT
}

\title{
Summary
}

This document is the final laboratory report for Tank 241-BY-101. Push mode core segments were removed from Risers 10B and 10D between May 27, 1997, and June 1, 1997. Segments were received and extruded at 222-S Laboratory. Analyses were performed in accordance with Tank 241-BY-10I Push Core Sampling and Analysis Plan (TSAP) (Bell, 1997) and Tank Safety Screening Data Quality. Objective (DQO) (Dukelow, et al., 1995).

None of the subsamples submitted for total alpha activity (AT) or differential scanning calorimetry (DSC) analysis exceeded the notification limits as stated in TSAP and DQO. The statistical results of the $95 \%$ confidence interval on the mean calculations are provided by the Tank Waste Remediation Systems (TWRS) Technical Basis Group, and are not considered in this report.

Near Infrared Spectroscopy (NIR) analysis was requested in order to compare NIR results with those obtained from percent water gravimetry analysis $(\% \mathrm{H} 2 \mathrm{O})$ and thermal gravimetric analysis (TGA). The TWRS Technical Basis Group rescinded the request for this analysis (Schreiber, 1997a), and neither NIR nor \% $\mathrm{H} 2 \mathrm{O}$ analyses were performed.

\section{Appearance and Sample Handling}

Two cores, each consisting of eight segments, were expected from Tank 241-BY-101. Complete cores were not obtained.

Attachment 1 illustrates subsamples generated in the laboratory for analysis and identifies their sources. This reference also relates tank farm identification numbers to their corresponding 222-S Laboratory sample numbers.

\section{Core 189}

Segments 1-3 were removed from Tank 241-BY-101, Riser 10B, between May 27, 1997, and May 28,1997 . All segments were received by 222-S Laboratory on May 30, 1997. Table 1 summarizes extrusion information.

Segment 3 was sampled using a Retained Gas Sampler (RGS) and extruded by the Process Chemistry and Statistical Analysis Group. 


\section{HNF-SD-WM-DP-258, REV. 0}

\section{Core 199}

Segments 1-2 were removed from Tank 241-BY-10I, Riser 10D, on May 28, 1997. All segments were received by 222-S Laboratory between May 29, 1997, and May 30, 1997. Table 2 summarizes extrusion information.

Segment 2 was sampled using a RGS and extruded by the Process Chemistry and Statistical Analysis Group.

\section{Field Blank}

A field blank was provided to 222-S Laboratory with Core 199. This sample was treated as a drainable liquid in accordance with TSAP. .

\section{Hydrostatic Head Fluid Blank}

A hydrostatic head fluid (HHF) blank (a lithium bromide solution), was provided to 222-S Laboratory with Core 199. This sample was analyzed by ion chromatography and inductively coupled plasma spectroscopy. 


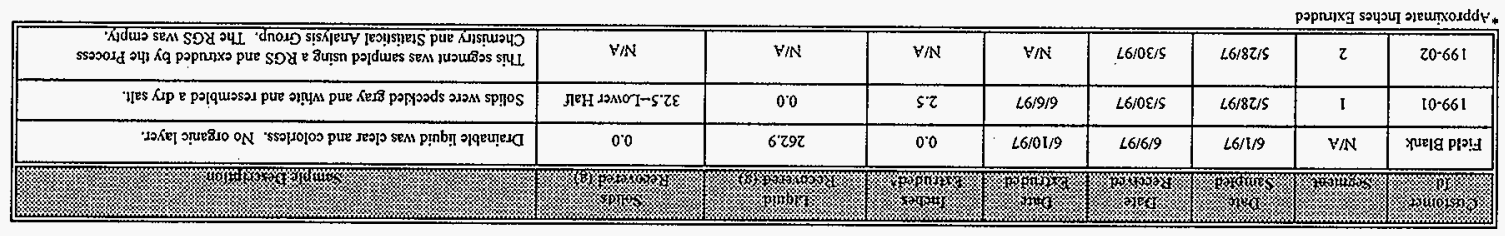

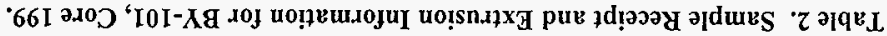

\begin{tabular}{|c|c|c|c|c|c|c|c|c|}
\hline 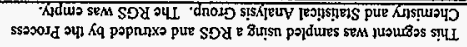 & $\forall / \mathbb{N}$ & $\forall / N$ & $V / N$ & $\forall / N$ & $26 / 0 \varepsilon / 5$ & $L 6 / L Z / S$ & $\varepsilon$ & $\varepsilon 0.68 \mathrm{I}$ \\
\hline 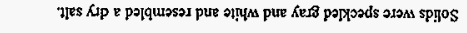 & 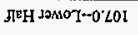 & 00 & $0 \varepsilon \varepsilon$ & $L 6 / 9 / 9$ & L6/0E/S & $L 6 / L Z / S$ & 2 & $20-68 \mathrm{I}$ \\
\hline 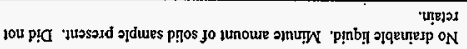 & 2015 & 00 & VIN & $L 6 / 9 / 9$ & $L 6 / 0 \varepsilon / S$ & $L 6 / L Z / S$ & I & $10 \cdot 681$ \\
\hline KY & 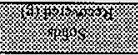 & 被 & \% & 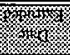 & 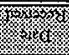 & 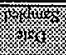 & 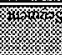 & \% \\
\hline
\end{tabular}

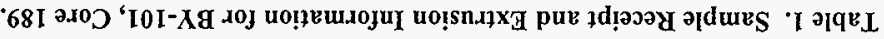

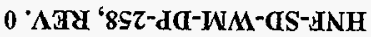




\section{HNF-SD-WM-DP-258, REV. 0}

\section{Analytical Results Summary}

The Data Summary Table (Table 3) included in this report compiles analytical results associated with each subsample submitted in accordance with TSAP and DQO.

Liquid subsamples prepared for analysis by an acid adjustment of the direct subsample are indicated by a "D" in the A\# column of Table 3. Solid subsamples prepared for analysis by performing a fusion digest are indicated by a "F" in the A\# column of Table 3. Solid subsamples prepared for analysis by performing a water digest are indicated by a "W" in the A\# column of Table 3.

\section{Inorganic Analyses}

\section{Differential Scanning Calorimetry (DSC) Analysis}

DSC analysis was performed on all direct subsamples. The exothermic energy based on dry weight of subsample was calculated for all subsamples. The average of the TGA results for each subsample was used in the dry weight correction for that subsample. All results were below DSC notification limits.

A second analysis of Lower Half Segment 1 of Core 199 (S97T001362) was performed (denoted by a "1" in the replicate (R) column of Table 3 ) was performed due to differences in appearance between the sample and duplicate. These differences were not seen in the replicate analyses. Results for original and replicate analyses are presented in Table 3. More information may be obtained by examining the raw data.

\section{Thermogravimetric Analysis (TGA)}

TGA was performed on all direct subsamples. TGA results were typically determined by summing weight loss steps below $200^{\circ} \mathrm{C}$. Weight loss steps above this were not used to determine the result. More information may be obtained by examining the raw data.

RPD between sample and duplicate for Lower Half Segment 1 of Core 199 (S97T001362) was 20.2. TGA results were near the detection level of the method, and precision was compromised. Rerun analysis was not requested.

\section{Density}

Bulk density was performed on solid half segments. There were no quality control (QC) parameters stated in TSAP for bulk density analysis.

Bulk denisty could not be determined for Lower Half Segment 1 of Core 199 (S97T001361) due to large void spaces in the bulk density tube. 


\section{HNF-SD-WM-DP-258, REV. 0}

\section{Specific Gravity (SpG)}

Specific gravity analyses were performed on direct liquid subsamples. There were no exceptions to the QC parameters stated in TSAP for these subsamples.

\section{Ion Chromatography (IC)}

IC analysis was performed on direct liquid and solid subsamples. For these subsamples, only bromide $\mathrm{Br}$ ) was reviewed for $\mathrm{QC}$ in accordance with TSAP. Results are included in Table 3. There were no exceptions to the QC parameters stated in TSAP for $\mathrm{Br}$.

All other analyte results are presented in Appendix A. These results are opportunistic, and do not have customer defined QC parameters. Any anomalies associated with the results are not discussed.

\section{Inductively Coupled Plasma Spectrophotometry (ICP)}

ICP was performed on direct liquid and solid subsamples. For these subsamples, only lithium (Li) was reviewed for $\mathrm{QC}$ in accordance with TSAP. Results are included in Table 3. There were no exceptions to the QC parameters stated in TSAP for $\mathrm{Li}$.

All other analyte results are presented in Appendix A. These results are opportunistic, and do not have customer defined QC parameters. Any anomalies associated with the results are not discussed.

\section{Radiochemistry Analyses}

\section{Total Alpha (AT) Analysis}

AT analyses were requested for direct liquid subsamples and solid lower half segments. All results were below the total alpha activity notification limits.

RPD between sample and duplicate for Lower Half Segment 1 of Core 199 (S97T001363) was 44.6. This was caused by counting uncertainties greater than 100\%. Spike recoveries for S97T001363 and Lower Half Segment 2 of Core 189 (S97T001358) were 65.8\% and 70.3\%, respectively. In both cases, the spike recovery was within method control limits for the standard. Rerun analyses were not requested. 


\section{HNF-SD-WM-DP-258, REV. 0}

\section{Procedures}

Table 4 lists the analytical procedures used for performing sample analyses. Abbreviations for analyses are defined in the table notes.

\section{Table 4. Analytical Procedures}

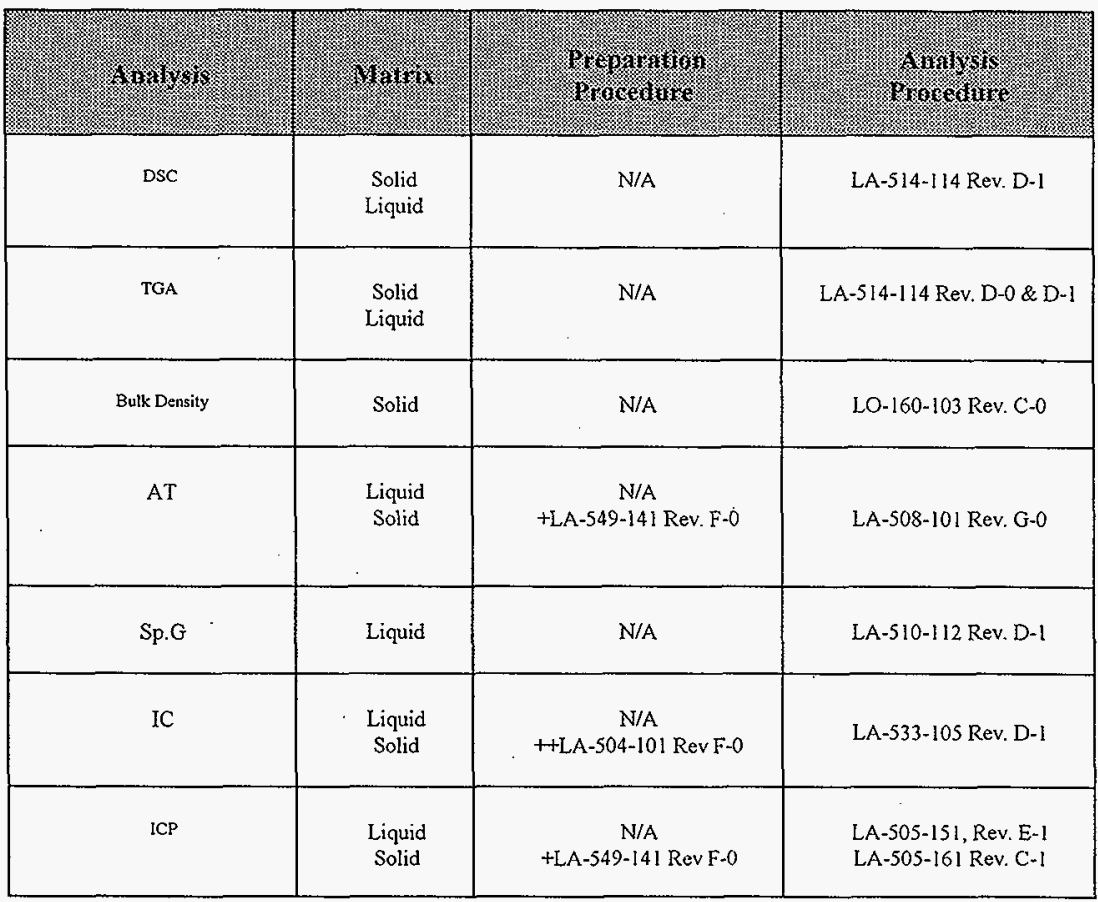


HNF-SD-WM-DP-258, REV. 0

Table Notes:

$\begin{array}{ll}\text { N/A } & \text { not applicable (these are direct samples) } \\ \text { DSC } & \text { differential scanning calorimetry } \\ \text { TGA } & \text { thermogravimetric analysis } \\ \text { AT } & \text { total alpha } \\ \text { Sp.G } & \text { specific gravity } \\ \text { IC } & \text { ion chromatography } \\ \text { ICP } & \text { inductively coupled plasma } \\ + & \text { fusion digest } \\ ++ & \text { water digest }\end{array}$




\section{HNF-SD-WM-DP-258, REV. 0}

\section{References}

Dukelow, G. T., J. W. Hunt, H. Babad, and J. E. Meacham, 1995, Tank Safety Screening Data Quality Objective, WHC-SD-WM-SP-004, Rev. 2, Westinghouse Hanford Company, Richland, WA 99352.

Bell, K.E., 1997, Tank 241-BY-101 Push Core Sampling and Analysis Plan, HNF-SD-WM-TSAP-128, Rev. 0, Lockheed Martin Hanford Corporation, Richland, WA 99352 .

Markel, L.P., 1997, 222-S Laboratory Quality Assurance Plan, HNF-SD-CP-QAPP-016, Revision 0, Waste Management Federal Services of Hanford, Richland, WA 99352

Schreiber, R. D. 1997a, Clarification Regarding Near Infrared Spectroscopy Analysis Request for Tank Waste Samples, Interoffice memo 74610-97-038 to B. A. Crawford, August 12, Lockheed Martin Hanford Company, Richland, WA 99352. 
HNF-SD-WM-DP-258, REV. 0

\section{BY-101 SAMPLE BREAKDOWN (ATTACHMENT 1)}


HNF-SD-WM-DP-258, REV. 0

THIS PAGE INTENTIONALLY LEFT BLANK

12 


\section{BY-101}

Core:189

Seg: 2 (189-02)

S97T001237

\section{ATTACHMENT 1}

\section{|}


HNF-SD-WM-DP-258, REV. 0
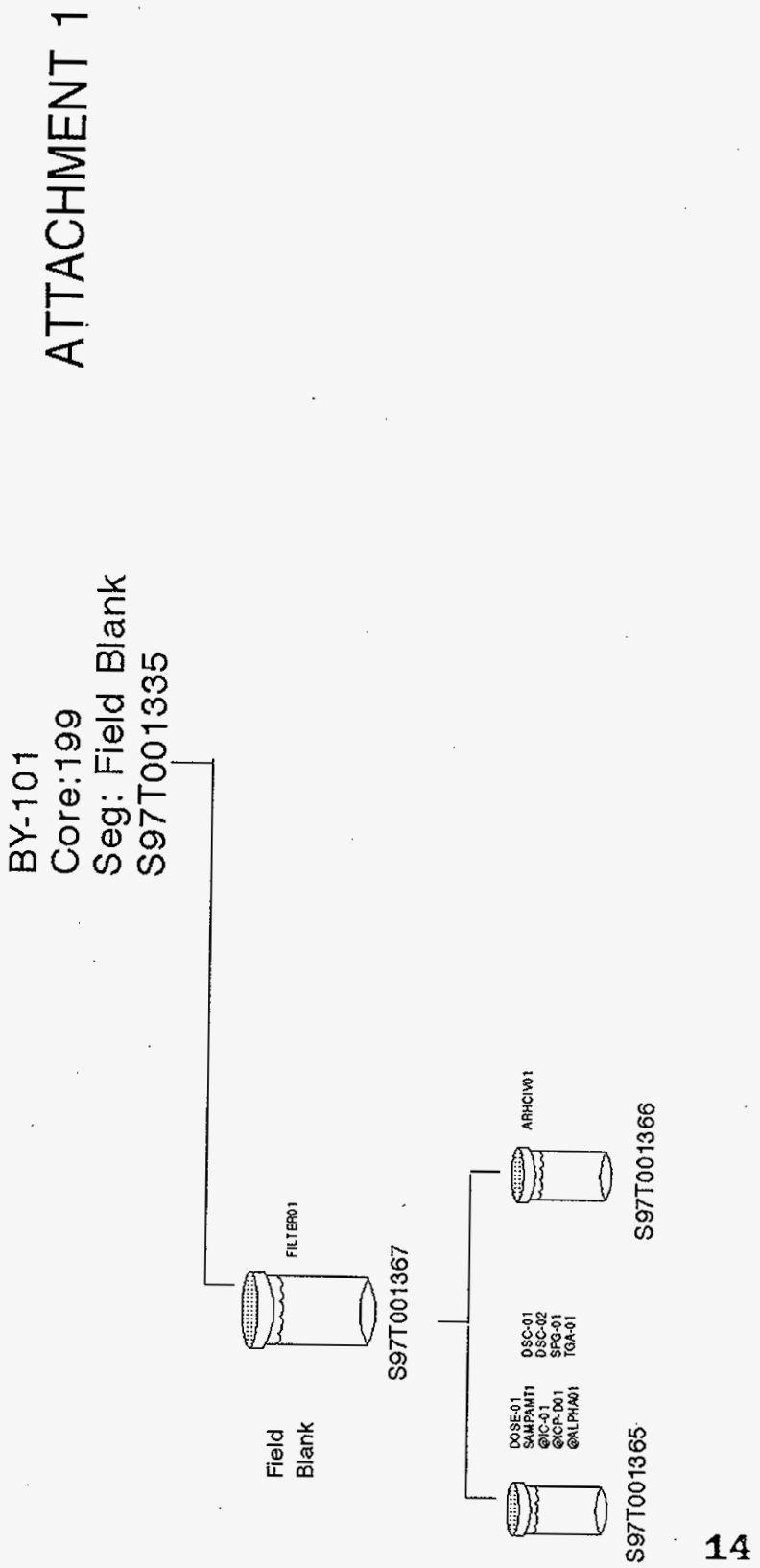
BY-101

Core:199

Seg: HHF (LiBr Blank)

S97T001241

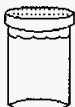

@|c.01

@ICP-D 01

er 
HNF-SD-WM-DP-258, REV. 0

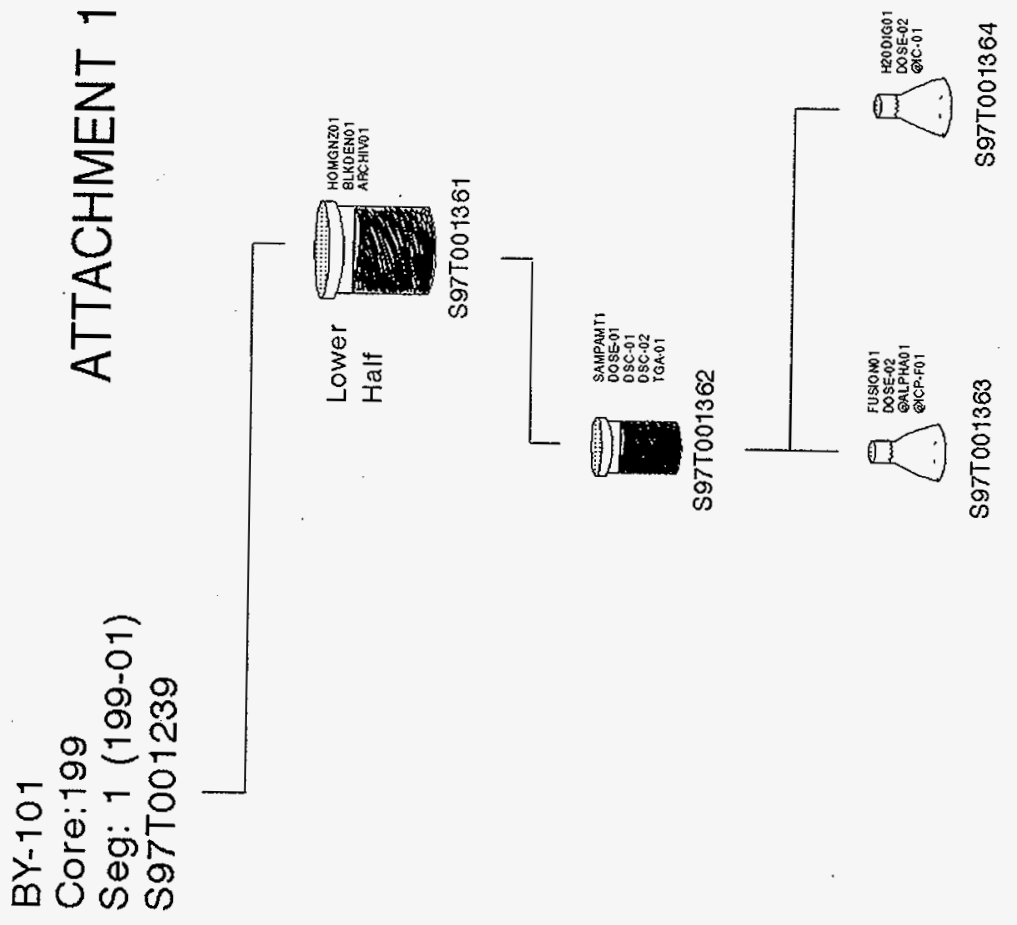


HNF-SD-HM-DP-258, REV. 0

SAMPLE DATA SUMMARY 
HNF-SD-WM-DP-258, REV. 0

THIS PAGE INTENTIONALLY LEFT BLANK 
HNF-SD-WM-DP-258, REV. 0

-
$\ddot{4}$
욤

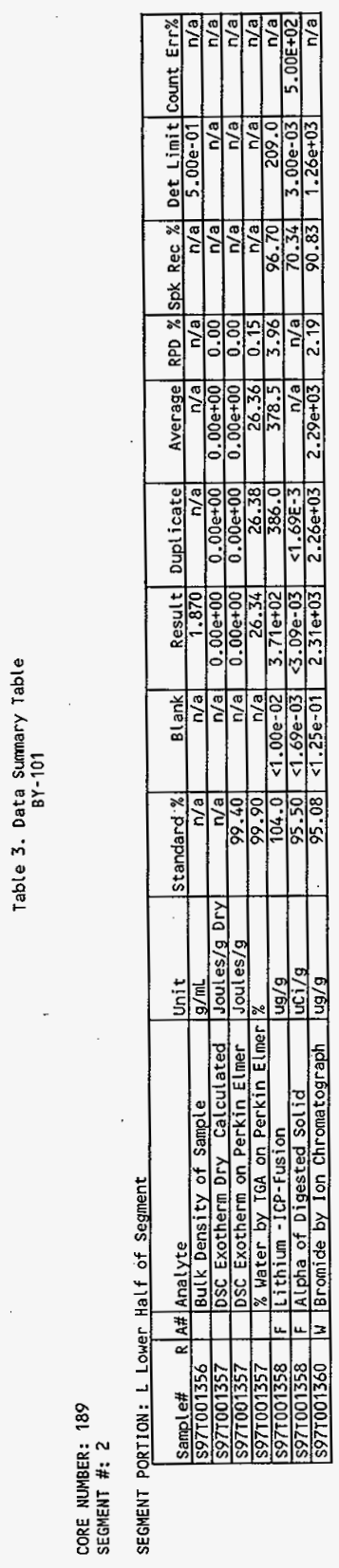


Table 3. Data Sumary Table

$$
\text { BY }-101
$$

CORE NUMBER: 190

SEGMENT \#: Field Blank

SEGMENT PORIION: Field Blank

\begin{tabular}{|c|c|c|c|c|c|c|c|c|c|c|c|c|}
\hline Sample\# $R$ & A\# & Analyte & Unit & Standard \% & $B \operatorname{Lan} k$ & Result & Dupl icate & Average & RPD \% & Spk Rec \% & Det Limit & Err\% \\
\hline 5977001365 & & DSC Exotherm Dry Calculated & Joules/g Dry & $n / a$ & $n / a$ & $0.00 \mathrm{e}+00$ & $0.00 \mathrm{e}+00$ & $0.00 \mathrm{e}+00$ & $\frac{\pi}{0.00}$ & $\frac{\mathrm{n} / \mathrm{a}}{\mathrm{sen} / \mathrm{k}}$ & $\mathrm{n} / \mathrm{a}$ & n/a \\
\hline S97T001365 & & DSC Exotherm on Perkin Elmer & Joules $/ \mathrm{g}$ & 99.40 & $n / a$ & $0.00 \mathrm{e}+00$ & $0.00 \mathrm{e}+00$ & $0.00 \mathrm{e}+00$ & 0.00 & $n / a$ & $n / a$ & $\mathrm{n} / \mathrm{a}$ \\
\hline $597 \mathrm{~T} 001365$ & & Specific Gravity & Sp.G. & 97.03 & $\mathrm{n} / \mathrm{a}$ & $9.60 \mathrm{e}-01$ & $9.72 e-01$ & $9.66 \mathrm{e}-01$ & 1.24 & $\mathrm{n} / \mathrm{a}$ & $1.00 \mathrm{e}-03$ & $n / a$ \\
\hline \begin{tabular}{|l|} 
S97T001365 \\
S97T001365
\end{tabular} & & $\%$ Water by TGA on Perkin Elmer & $\%$ & 99.90 & $\mathrm{n} / \mathrm{a}$ & 99.48 & 99.47 & 99.47 & 0.01 & $\mathrm{n} / \mathrm{a}$ & $\mathrm{n} / \mathrm{a}$ & n/a \\
\hline S97T001365 & D & Lithium-ICP-ACid Dil. & $\mathrm{ug} / \mathrm{mL}$ & 105.0 & $<1.00 \mathrm{e}-02$ & $1.16 \mathrm{e}-01$ & $1.15 \mathrm{e}-01$ & $1.16 e^{-01}$ & 0.87 & n/a & $2.00 e^{-02}$ & $n / a$ \\
\hline S97T001365 & & Bromide by Ion Chromatograph & $\mathrm{ug} / \mathrm{mL}$ & 95.76 & $<1.25 \mathrm{e}-01$ & 1.146 & 1.140 & 1.143 & 0.87 & $\mathrm{n} / \mathrm{a}$ & $1.25 e-01$ & $\mathrm{n} / \mathrm{a}$ \\
\hline \$97T001365 & & Alpha in Liquid Samples & $\mathrm{uCj} / \mathrm{mL}$ & 91.50 & $<7.81 \mathrm{e}-07$ & $<7.81 \mathrm{e}-07$ & $<7.81 \mathrm{E}-7$ & $n / a$ & $\mathrm{n} / \mathrm{a}$ & $\mathrm{n} / \mathrm{a}$ & $1.87 e-06$ & $5.00 E+02$ \\
\hline
\end{tabular}


Table 3. Data Summary Table BY-101

CORE NUMBER: 199

SEGMENT \#: $n / a$

SEGMENT PORTION: Hydrostatic Head Fluid

\begin{tabular}{|c|c|c|c|c|c|c|c|c|c|c|c|c|}
\hline Sample\# & A A\# & Analyte & Unit & Standard \% & B l.ank & Result & Duplicate & Average & $\mathrm{RPD} \%$ & Spk ReC \% & Det Limit & Err\% \\
\hline 5977001241 & D & Lithium-ICP-Acid Dil. & $\mathrm{ug} / \mathrm{mL}$ & 99.00 & $<1.00 e-02$ & $2.18 \mathrm{e}+03$ & $2.17 \mathrm{e}+03$ & $2.18 \mathrm{e}+03$ & 0.46 & $n / a$ & $2.00 \mathrm{e}-01$ & $n / a$ \\
\hline S971001241 & & Bromide by Ion Chromatograph & $\mathrm{ug} / \mathrm{mL}$ & 103.2 & $<1.25 \mathrm{e}-01$ & $2.43 \mathrm{e}+04$ & $2.48 \mathrm{e}+04$ & $2.46 \mathrm{e}+04$ & 2,04 & n/a & 138.9 & \\
\hline
\end{tabular}


Table 3. Data Sumary Table BY- 101

CORE NUMBER: 199

SEGMENT \#:

SEGMENT PORIION: L. Lower Half of Segment

\begin{tabular}{|c|c|c|c|c|c|c|c|c|c|c|c|c|}
\hline Sample\# & A\# & Analyte & Unit & Standard \% & B lank & Result & Duplicate & Average & RPD \% & Spk Rec \% & Limit & Eгг\% \\
\hline 597T001361 & & Bulk Density of Sample & $\mathrm{g} / \mathrm{mL}$ & $\mathrm{n} / \mathrm{a}$ & $\mathrm{n} / \mathrm{a}$ & na & $n / a$ & $\mathrm{n} / \mathrm{a}$ & $\mathrm{n} / \mathrm{a}$ & $n / a$ & $5.00 \mathrm{e}-01$ & $\mathrm{n} / \mathrm{a}$ \\
\hline \$97T001362 & & DSC Exotherm Dry Calculated & Joules/g Dry & $n / a$ & $n / a$ & 54.70 & $0.00 \mathrm{e}+00$ & 27.35 & 200 & $\mathrm{n} / \mathrm{a}$ & $n / a$ & $\mathrm{n} / \mathrm{a}$ \\
\hline $597 T 0013621$ & & DSC Exotherm Ory Calculated & Joules/g Dry & $\mathrm{n} / \mathrm{a}$ & $\mathrm{n} / \mathrm{a}$ & $0.00 e+00$ & $0.00 \mathrm{e}+00$ & $0.00 \mathrm{e}+00$ & 0.00 & $n / a$ & $n / a$ & $\mathrm{n} / \mathrm{a}$ \\
\hline S97T001362 & & DSC Exotherm on Perkin Elmer & Joules/g & 96.66 & $n / a$ & 44.27 & $0.00 \mathrm{e}+00$ & 22.14 & 200 & $n / a$ & $n / a$ & $n / a$ \\
\hline \$97T001362 1 & & DSC Exotherm on Perkin Elmer & Joules/g & 101.8 & $n / a$ & $0.00 \mathrm{e}+00$ & $0.00 \mathrm{e}+00$ & $0.00 \mathrm{e}+00$ & 0.00 & $\mathrm{n} / \mathrm{a}$ & $n / a$ & $\mathrm{n} / \mathrm{a}$ \\
\hline$\$ 97 T 001362$ & & $\%$ Water by TGA on Perkin Elmer & $\%$ & 98.49 & $\mathrm{n} / \mathrm{a}$ & 17.14 & 21.00 & 19.07 & 20.2 & $n / a$ & $\mathrm{n} / \mathrm{a}$ & $n / a$ \\
\hline 5977001363 & $F$ & Lithium -ICP-Fusion & $u g / g$ & 904.0 & $<1.00 \mathrm{e}-02$ & $<2.04 \mathrm{e}+02$ & $<2.01 \mathrm{e} 2$ & $\mathrm{n} / \mathrm{a}$ & $n / a$ & 98.20 & 204.0 & $n / a$ \\
\hline $597 T 001363$ & $F$ & Alpha of Digested Solid & $u C \mathrm{i} / \mathrm{g}$ & 95.50 & $<1.69 \mathrm{e}-03$ & $2.74 \mathrm{e}-03$ & $1.74 e-03$ & $2.24 \mathrm{e}^{-03}$ & 44.6 & 65.78 & $00 \mathrm{e}-03$ & $06 E+02$ \\
\hline S97T001364 & W & Bromide by Ion Chromatograph & ug/g & 95.08 & $<1.25 e^{-01}$ & $<1.29 \mathrm{e}+03$ & $<1.26 \mathrm{e} 3$ & $n / a$ & $n / a$ & 97.28 & $29 \mathrm{e}+03$ & $n / a$ \\
\hline
\end{tabular}


HNF-SD-WM-DP-258, REV. O

CHAIN OF CUSTODY FORMS 
HNF-SD-HM-DP-258, REV. 0

THIS PAGE INTENTIONALLY LEFT BLANK

24 
CHAIN-OF-CUSTODY RECORD FOR CORE SAMPLING

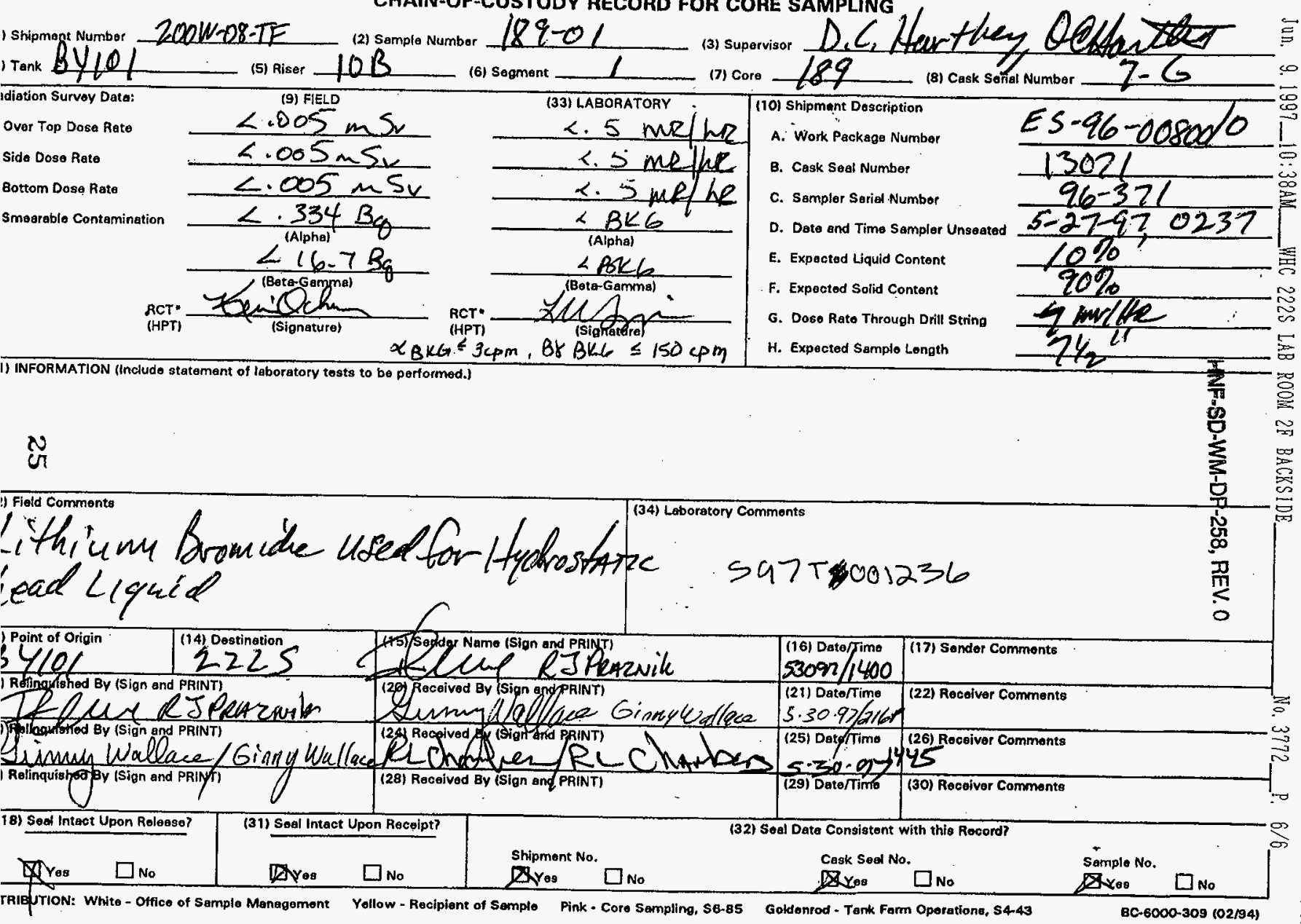


CHAIN-OF-CUSTODY RECORD FOR CORE SAMPLING

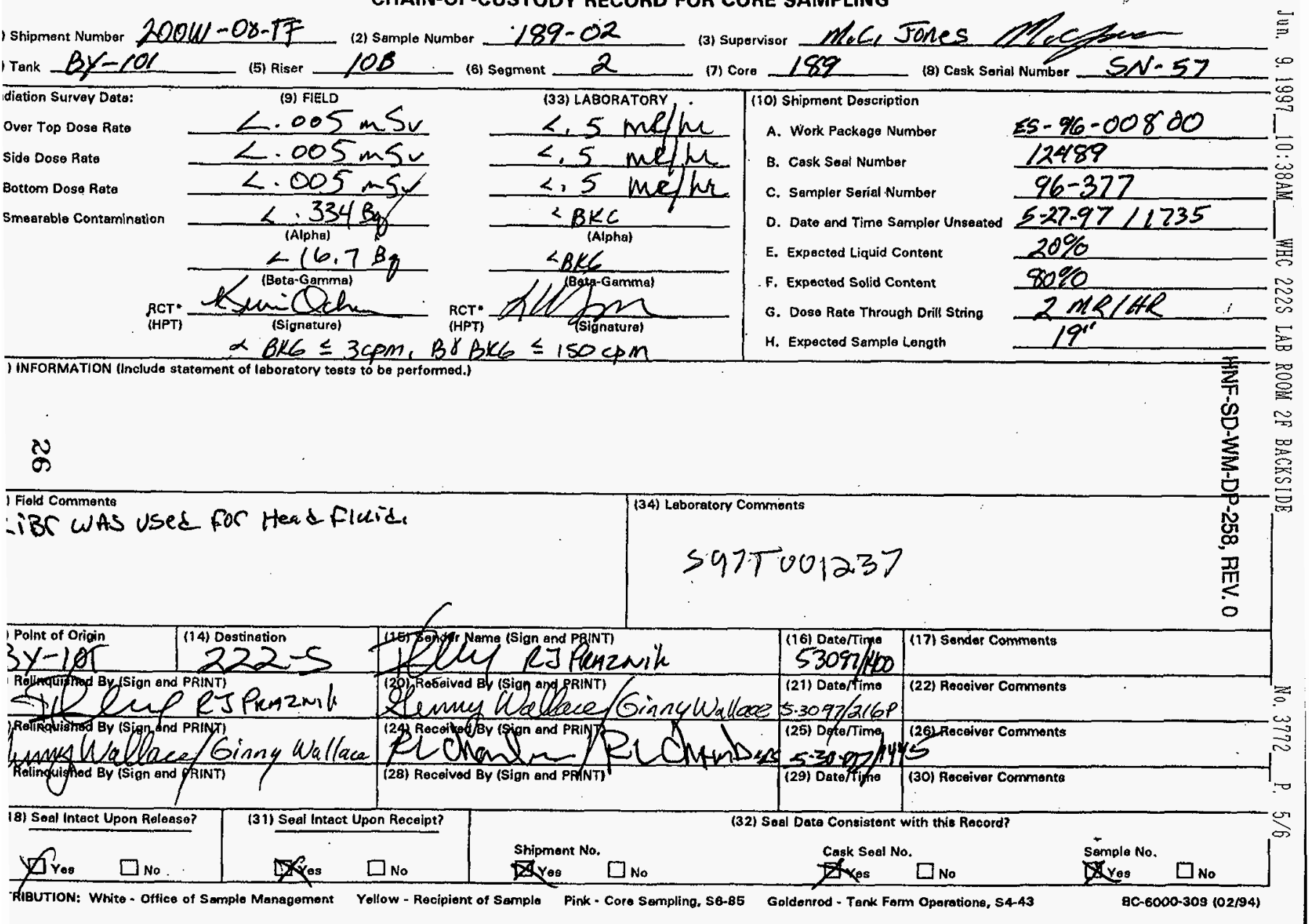




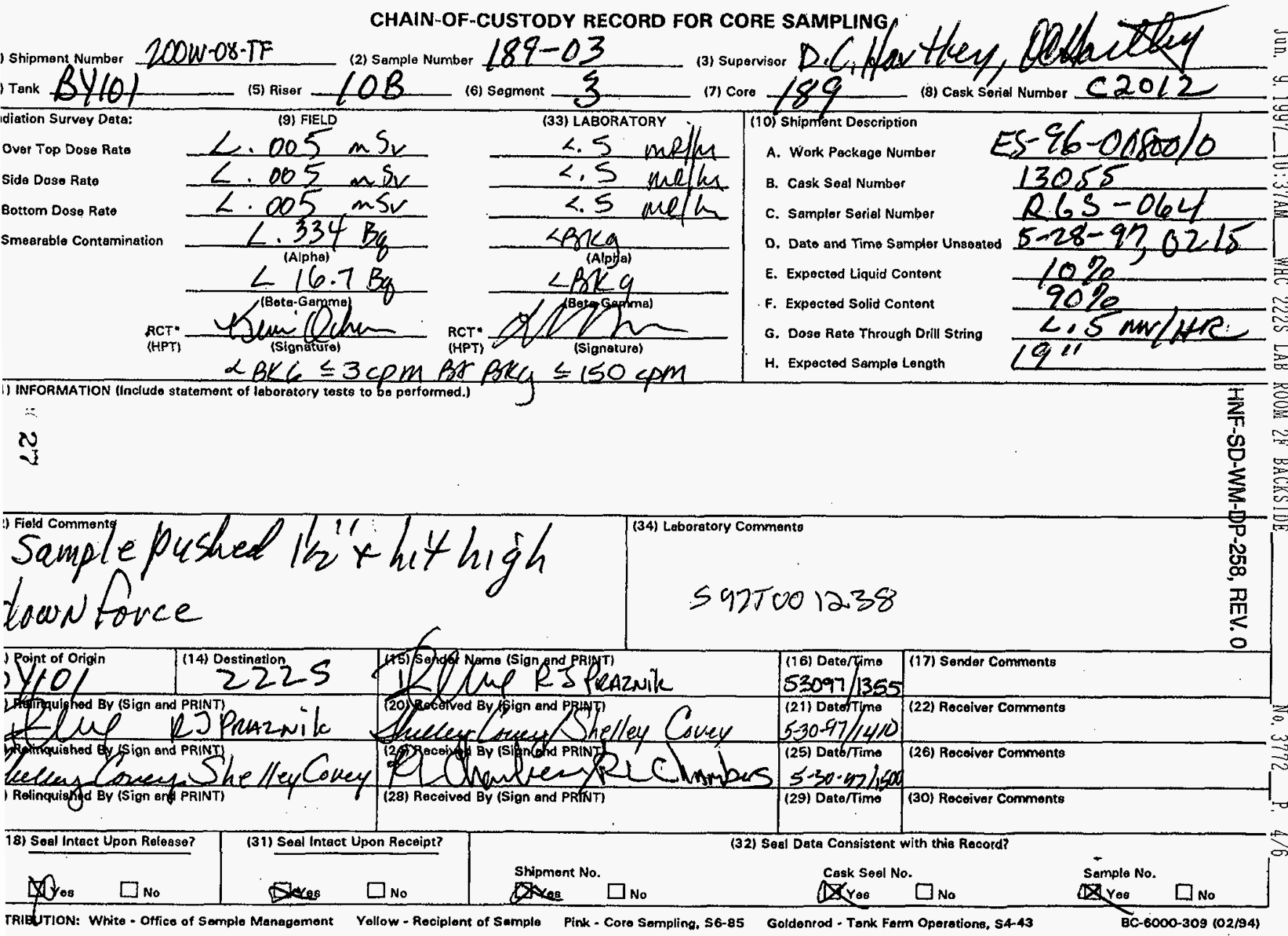


CHAIN-OF-CUSTODY RECORD FOR CORE SAMPLING

(1) Shiprnont Number 200 $00-08-T F$ (2) Sampla Numbar Field BlaNk (3) Suparvisor Men Jones (4) Tank $B Y-101$ (5) Alsor 100 (a) sagniont $-N / A$ (7) coro 199 (8) Cask Sorlal Numbor $\operatorname{sen-5c}$

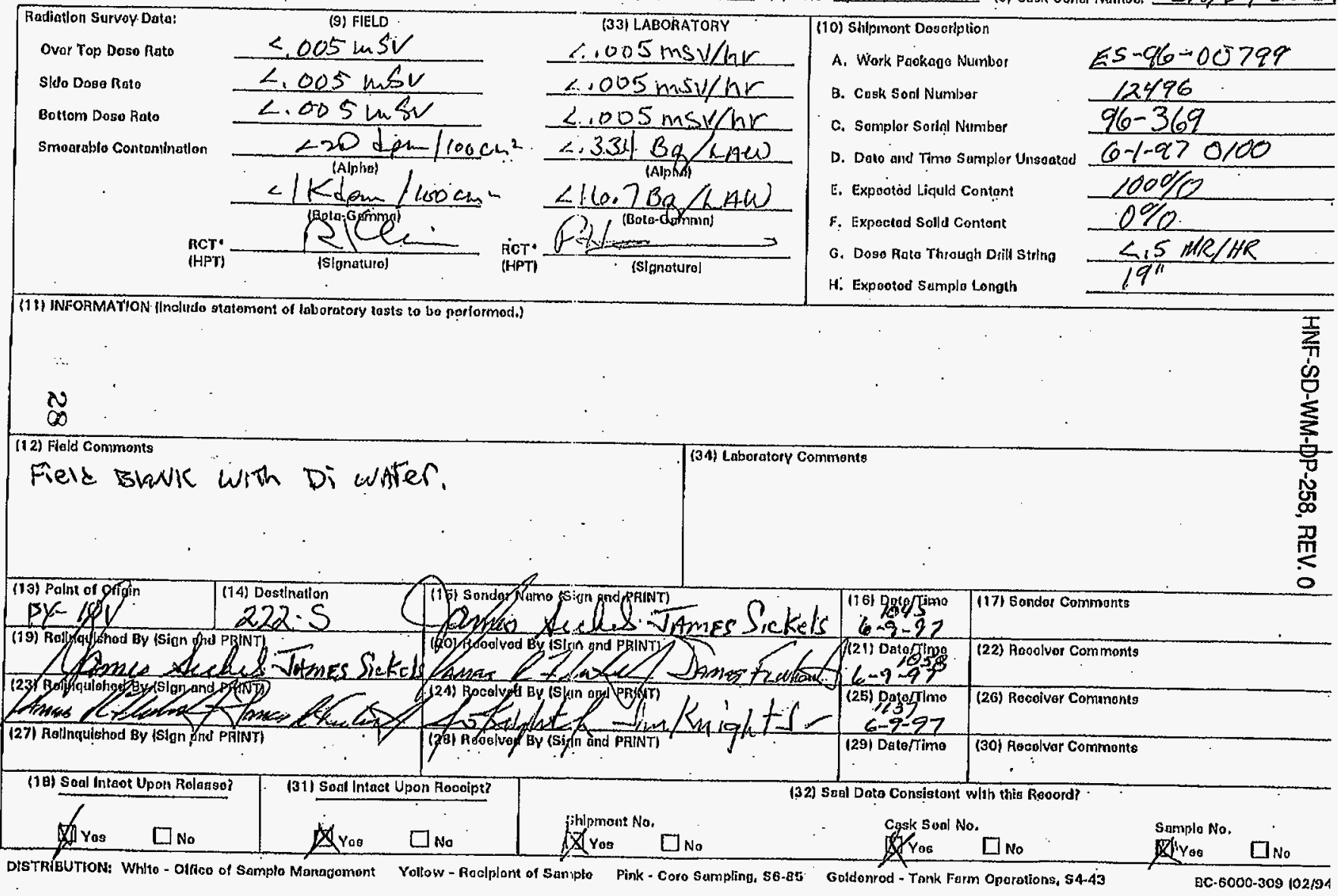


CHAIN-OF-CUSTODY RECORD FOR CORE SAMPLING

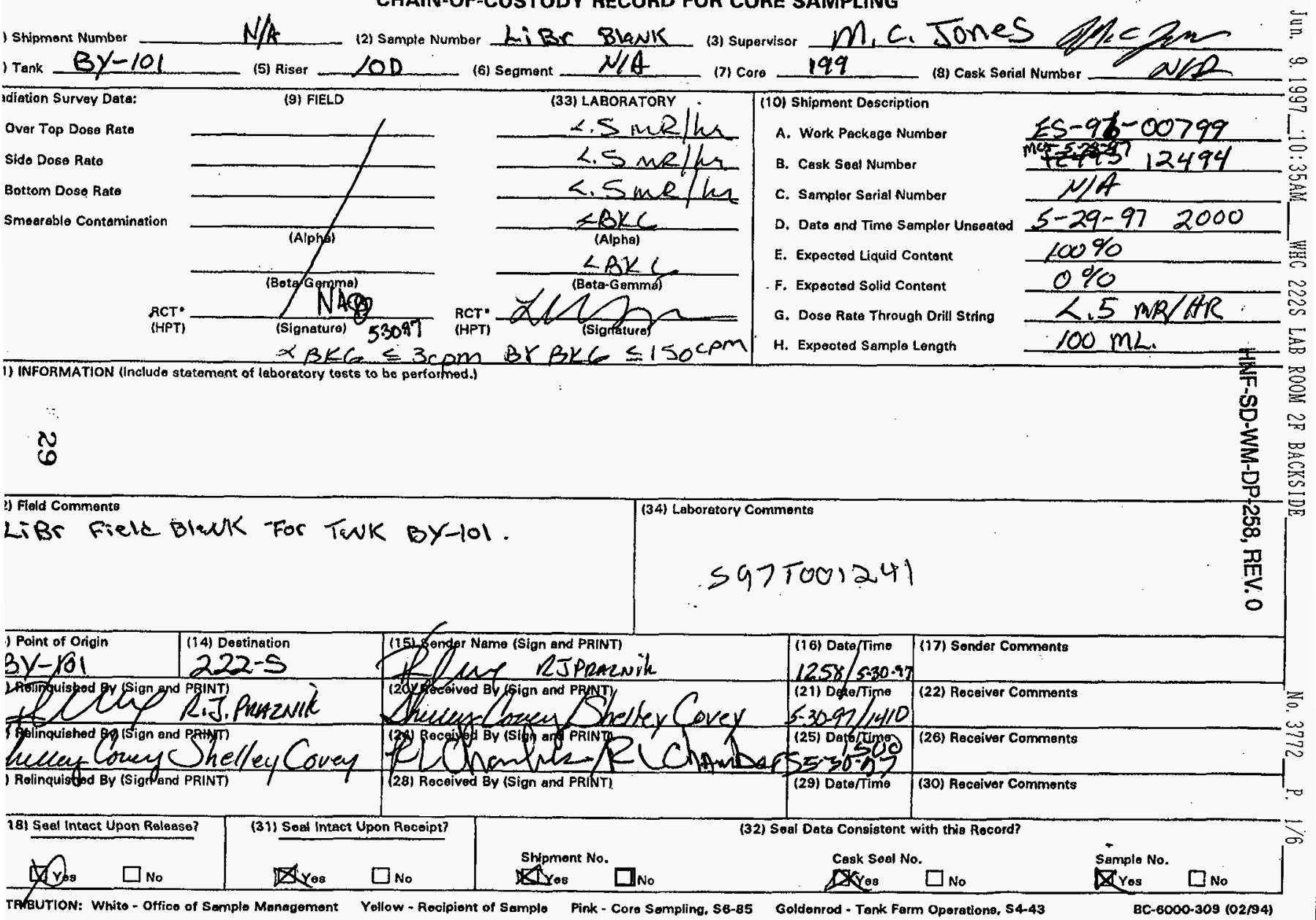


HNF-SD-HM-DP-258, REV , 0

PHOTOGRAPHS 
HNF-SD-HM-DP-258, REV. O

THIS PAGE INTENTIONALLY LEFT BLANK 
HNF-SD-WM-DP-258, REV. 0

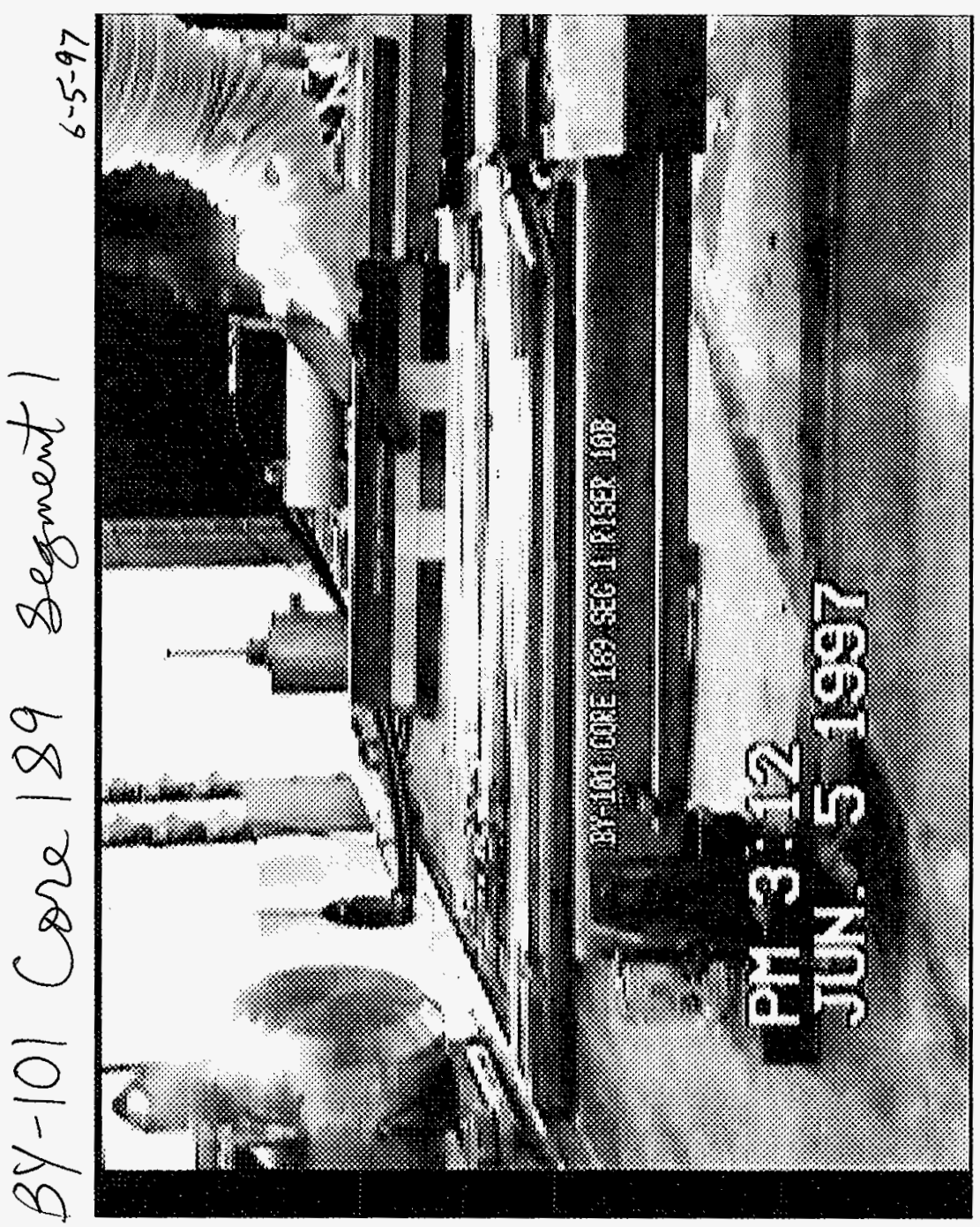





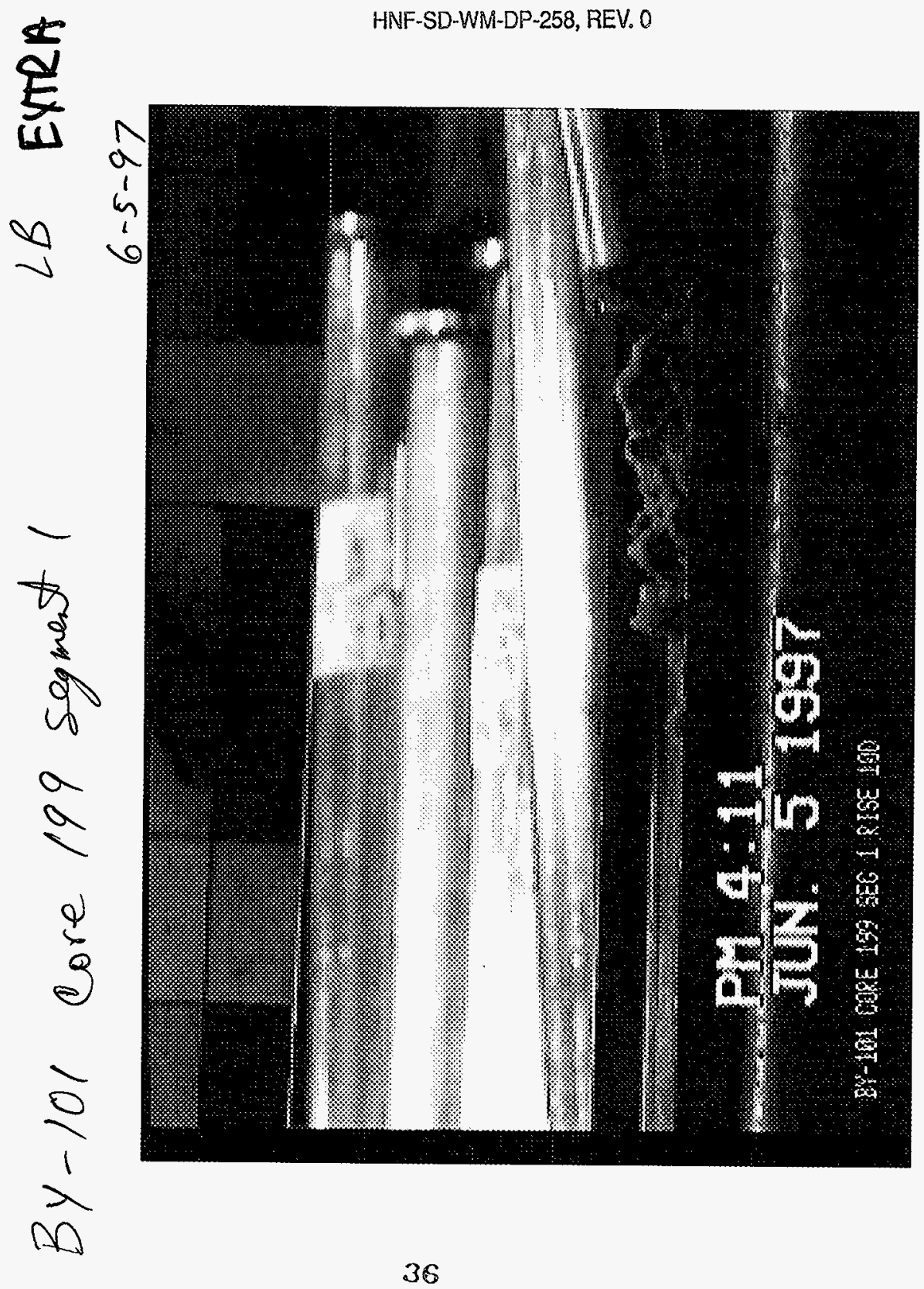


HNF-SD-WM-DP-258, REV. 0

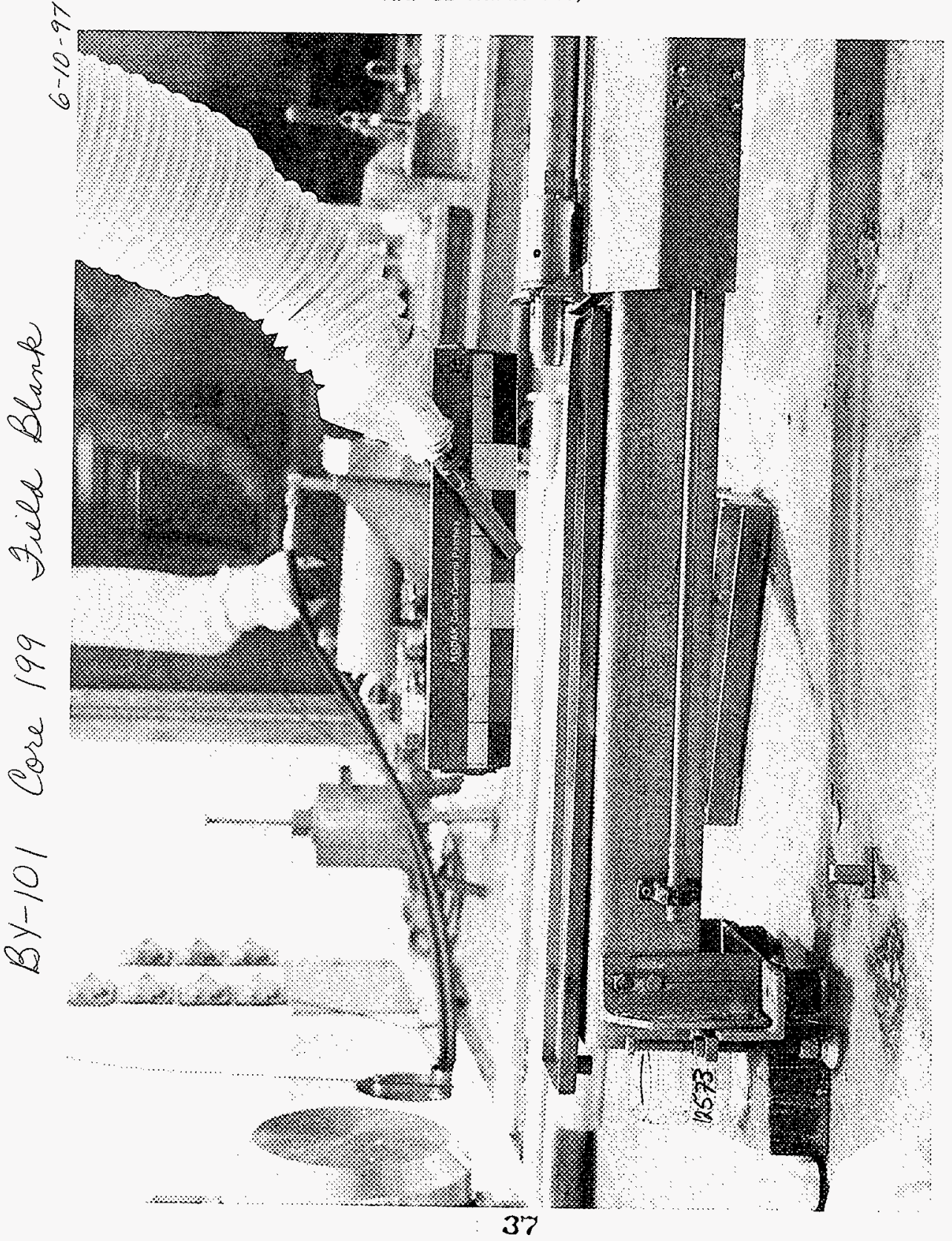


HNF-SD-HM-DP-258, REV. 0

SAMPLE HANDLING 
HNF-SD-HM-DP-258, REV. 0

THIS PAGE INTENTIONALLY LEFT BLANK 


\section{LABCORE Data Entry Template for Worklist\# 18452}

Analyst:

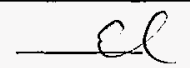

Instrument: BA000

Book \# NR

Method: LO-160-103 Rev/Mod

BO

\section{Worklist Comment: BY-101 C-199 SEG \#1 RISER 10D EXTRUSION}

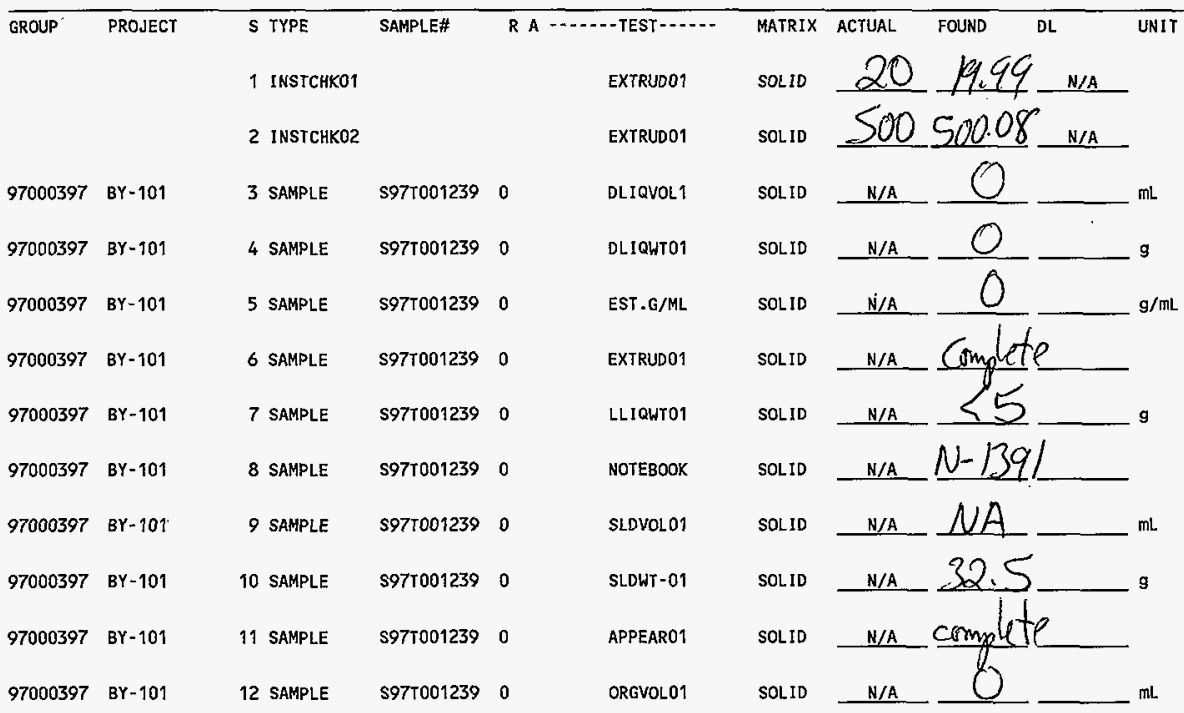

\section{Final page for worklist \# 18452}
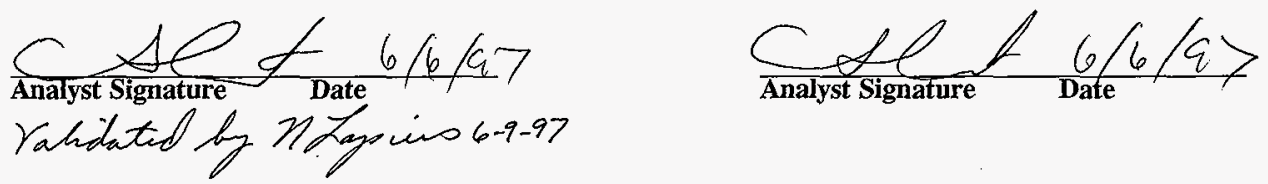

Data Entry Comments:

Units shown for $Q C$ (SPK \& STD) may not reflect the actual units. $D L=$ Detection Limit, $S=$ Worklist Slot Number,

$R=$ Replicate Number, $A=$ Aliquot Code. 


\section{LABCORE Data Entry Template for Worklist\# 18454}

Analyst: $\quad \varepsilon C$ Instrument: BA000 _ Book \# NA

Method: LO-160-103 Rev/Mod_BO

Worklist Comment: BY-101 C-189 SEG \#1 RISER 10B

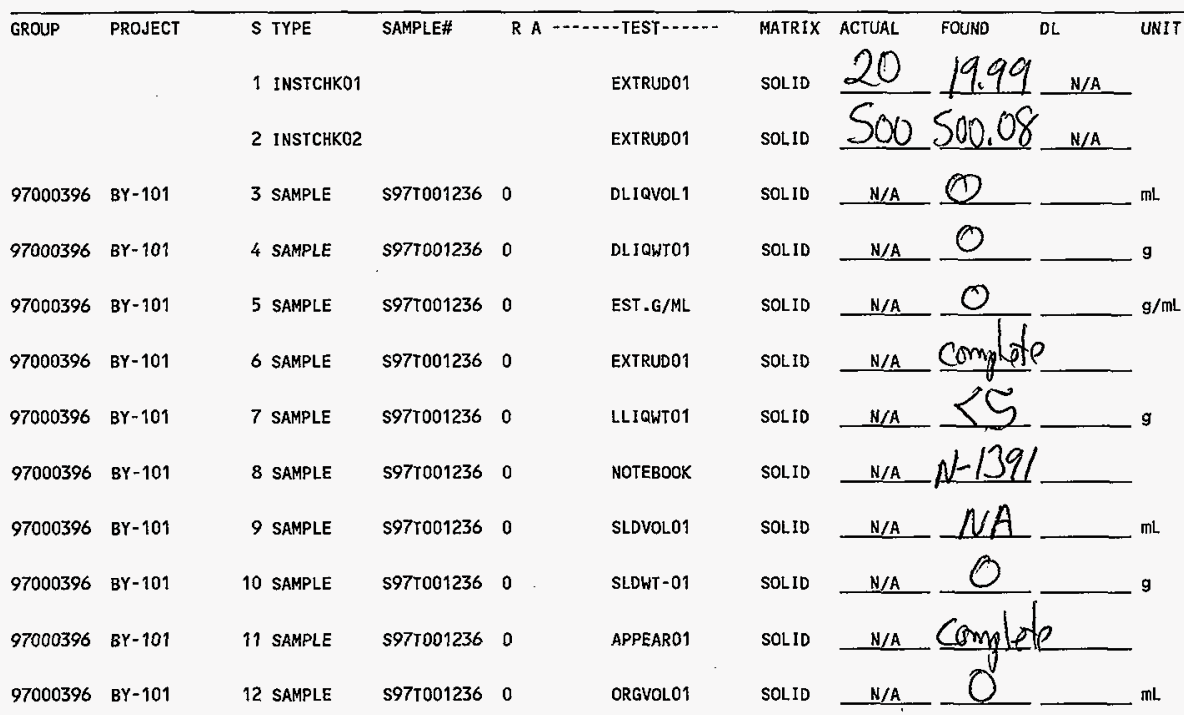

Final page for worklist \# 18454

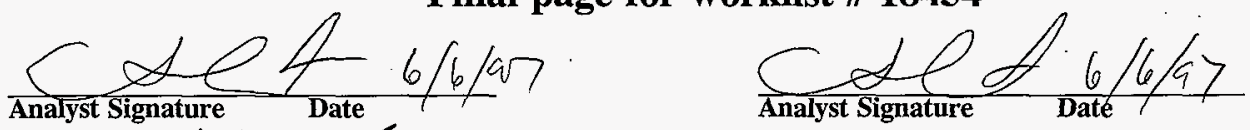

ralidated hy 17 Tapins 6-9-97 . 


\section{LABCORE Data Entry Template for Worklist\# 18456}

Analyst: $\quad E C$ Instrument: BA000_ Book\# NA

Method: LO-160-103 Rev/Mod B O

Worklist Comment: BY-101 C-189 SEG \#2 RISER 10B

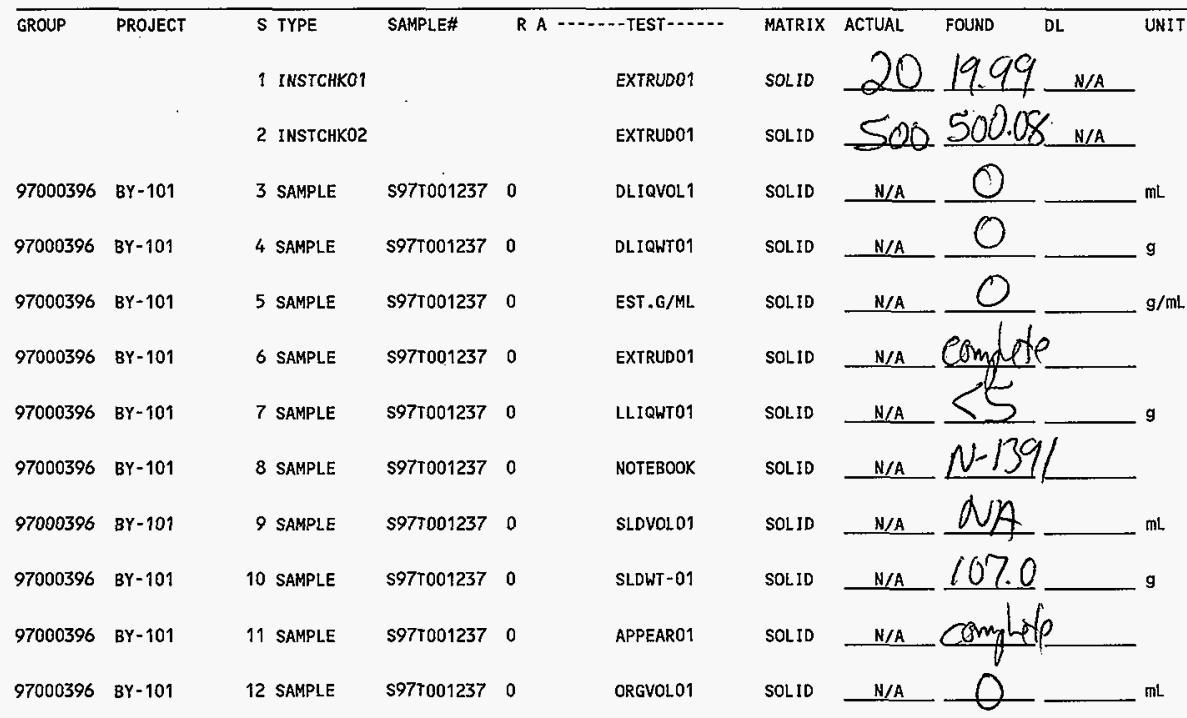

\section{Final page for worklist \# 18456}
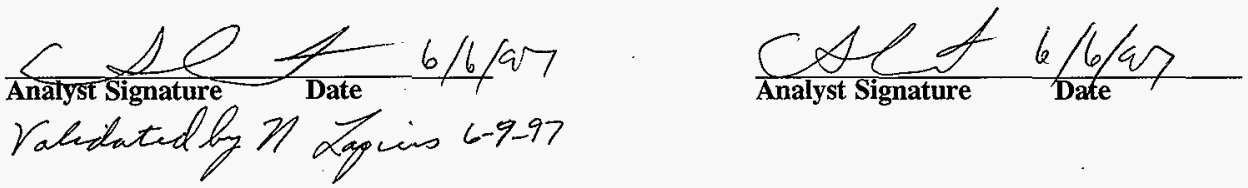

Data Entry Comments:

Units shown for $Q C$ (SPK \& STD) may not reflect the actual units. $D L=$ Detection Limit, $S=$ Worklist Slot Number, $\boldsymbol{R}=$ Replicate Number, $\boldsymbol{A}=$ Aliquot Code. 


\section{LABCORE Data Entry Template for Worklist\# 18573}

Analyst: $\quad$ Instrument: BA000 B Book\# NA

Method: LO-160-103 Rev/Mod

乃O

Worklist Comment: BY-101 C199 FIELD BLANK RISER 10D EXTRUSION

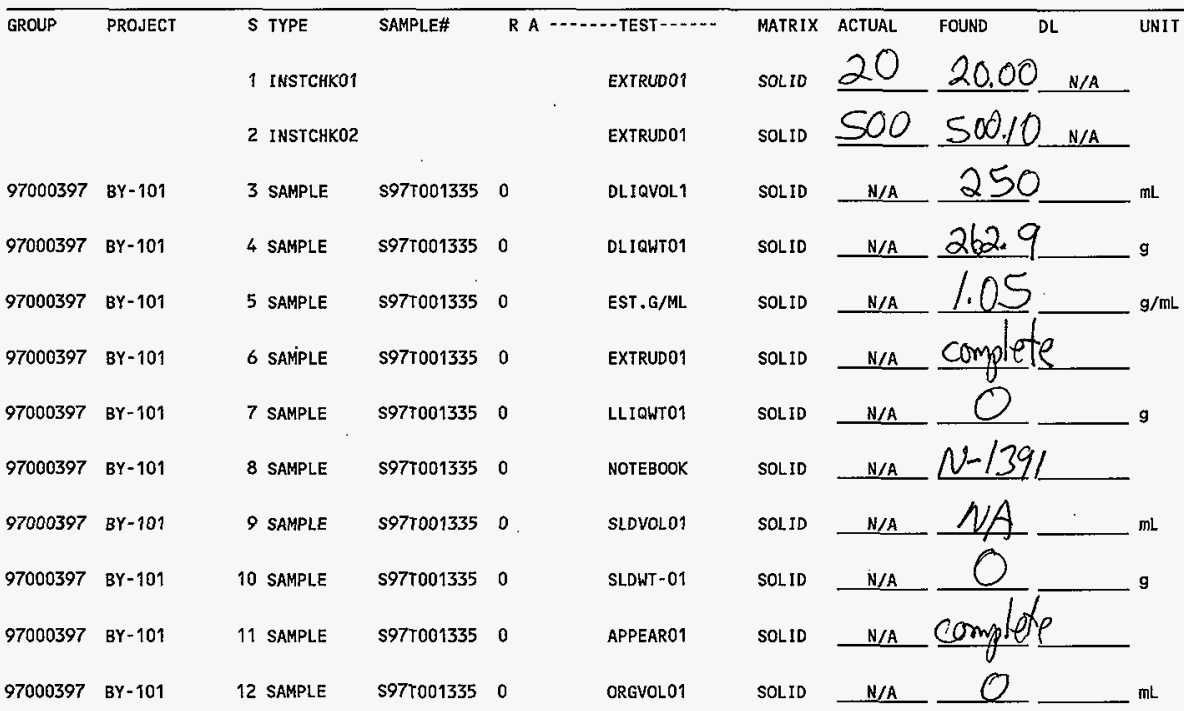

\section{Final page for worklist \# 18573}
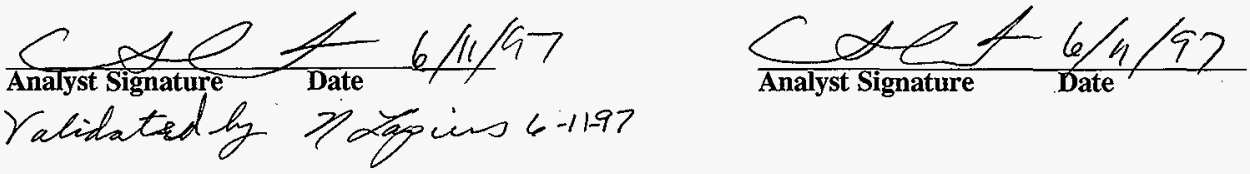

Data Entry Comments:

Units shown for QC (SPK \& STD) may not reflect the actual units. $D L=$ Detection Limit, $S=$ Worklist Slot Number,

$R=$ Replicate Number, $A=$ Aliquot Code. 
HNF-SD-WM-DP-258, REV. 0

SAMPLE PREPARATIONS

44 
HNF-SD-HM-DP-258, REV. 0

THIS PAGE INTENTIONALLY LEFT BLANK 


\section{LABCORE Data Entry Template for Worklist\# 18696}

$\begin{array}{lll}\text { Analyst: } \quad \text { DCD } & \text { Instrument: FUS01 } & \text { Book \# N/A } \\ \text { Method: LA-549-141 Rev/Mod } & \text { F-O } & \end{array}$

Worklist Comment: BY-101 FUSION DIGEST C189S2 C199S1 lad

\begin{tabular}{|c|c|c|c|c|c|c|c|c|c|c|}
\hline GROUP & PROJECT & $S$ TYPE & SAMPLE\# & $R A-$ & - TEST $\cdots$ & MATRIX & ACTUAL & FOUND & DL & UNIT \\
\hline & & 1 BL.NK-PREP & & & FUSIONO1 & SOLID & 1 & 0.250 & N/A & $g / L$ \\
\hline 97000396 & BY -101 & 2 SAMPLE & s97T001358 & $O F$ & FUSIONOT & SOLID & N/A & 1.964 & & $.9 / L$ \\
\hline 97000396 & BY-101 & 3 SAMPLE & s97T001358 & $O F$ & DOSE-02 & SOLID & N/A & 2.5 & & $\mathrm{mrad} /$ hour \\
\hline 97000396 & $\mathrm{BY}-101$ & $\begin{array}{l}4 \text { DUP } \\
0.4900 \mathrm{~g}\end{array}$ & $\begin{array}{l}s 977001358 \\
\rightarrow 0.25\end{array}$ & $\begin{array}{c}0 \mathrm{~F} \\
\infty\end{array}$ & FUSION01 & SOLID & 1.964 & 1.960 & N/A & $g / L$ \\
\hline 97000396 & $B Y-101$ & 5 DUP & s97T001358 & $0 \mathrm{~F}$ & DOSE-02 & SOLID & 2.5 & 2.5 & N/A & mrad/hour \\
\hline 97000397 & $B Y-101$ & 6 SAMPLE & s97to01363 & $0 \mathrm{~F}$ & FUSIONO1 & SOLID & N/A & 2.0108 & & $g / L$ \\
\hline 97000397 & BY-101 & $\underset{7 \text { SAMPLE }}{0.5027} 9$ & $\begin{array}{l}\rightarrow 0.25 \\
\text { S97T001363 }\end{array}$ & ${ }_{0}^{\circ} \underset{F}{e}$ & DOSE-02 & SOLID & N/A & 1.5 & & mrad/hour \\
\hline 97000397 & BY-101 & 8 DUP & S97T001363 & $0 \mathrm{~F}$ & FUSIONOT & SOLID & $2.0 \vee 08$ & 2.0424 & N/A & $-g / L^{\circ}$ \\
\hline 97000397 & BY-101 & $0_{9 \text { DUP }}^{0.51069}$ & $\rightarrow 0.25$ & ${ }_{0}^{\circ} \mathcal{F}$ & DOSE -02 & SOLID & 1.5 & 1.5 & N/A & mrad/hour \\
\hline
\end{tabular}

\section{Final page for worklist \# 18696}

\section{Danded Sumberm $7-19-97$}

Analyst Signature Date

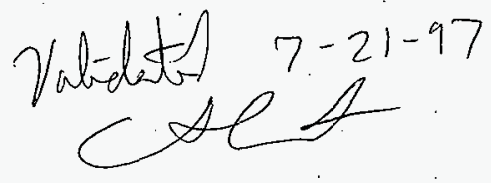

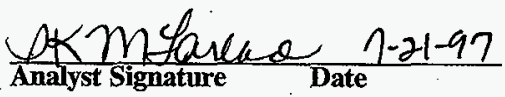

\section{Data Entry Comments:}

HPT surveged by Joe Valdez 7/18/97 Added $40 \mathrm{mls}$ of $\mathrm{HNO}_{3}$.

Units shown for $Q C$ (SPK \& STD) may not reflect the actual units. $D L=$ Detection Limit, $S=$ Worklist Slot Number, $R=$ Replicate Number, $A=$ Aliquot Code. 


\section{LABCORE Data Entry Template for Worklist\# 18697}

Analyst: $\quad$ Instrument: H2O01

Book \# $N / A$

Method: LA-504-101 Rev/Mod F.O

Worklist Comment: BY-101 H20 DIGEST C189S2 C199S1 lad

\begin{tabular}{|c|c|c|c|c|c|c|c|c|c|c|}
\hline GROUP & PROJECT & $\begin{array}{l}S \text { TYPE } \\
1 \text { BLNK-PREP }\end{array}$ & SAMPLE\# & RA $\cdots$ & H2001G01 & $\begin{array}{l}\text { MATRIX } \\
\text { SOLID }\end{array}$ & $\begin{array}{r}\text { ACTUAL } \\
1 \\
\end{array}$ & $\begin{array}{l}\text { FOUND } \\
0.100 \\
\end{array}$ & $\begin{array}{l}\mathrm{DL} \\
\mathrm{N} / \mathrm{A} \\
\end{array}$ & $\begin{array}{l}\text { UNIT } \\
g / L\end{array}$ \\
\hline 97000396 & $B Y-101$ & 2 SAMPLE & s97T001360 & $\mathrm{OH}$ & $\mathrm{H} 2001601$ & SOLID & N/A & 5.061 & & $g / L$ \\
\hline 97000396 & $B Y-101$ & $\begin{array}{l}0.506 / \\
3 \text { SAMPLE }\end{array}$ & $g_{S 97 T 001360}^{\rightarrow} 0$ & $\begin{array}{l}.100 \mathrm{e} \\
.0 \mathrm{~W}\end{array}$ & DOSE-02 & SOLID & N/A & 1 & & $\mathrm{mrad} /$ hour \\
\hline 97000396 & BY-101 & 4 DUP & s97T001360 & $\mathrm{OH}$ & $\mathrm{H} 2001601$ & SOLID & 5.061 & 5.001 & N/A & g/L \\
\hline 97000396 & BY-101 & $\begin{array}{l}0.500 / \\
5 \text { DUP }\end{array}$ & $g \rightarrow \underset{s 97 T 001360}{\rightarrow}$ & $100 l$ & DOSE-02 & SOLID & 1 & 1 & N/A & mrad/hour \\
\hline 97000397 & BY-101 & 6 SAMPLE & S97T001364 & $\mathrm{OW}$ & H2ODIG01 & SOLID & $\mathrm{N} / \mathrm{A}$ & $7.9 \%$ & & $g / L$ \\
\hline 97000397 & BY-101 & $\begin{array}{c}0.4948 \mathrm{~g} \\
7 \text { SAMPLE }\end{array}$ & $\rightarrow 0.100$ & $\frac{l}{0 \mathrm{H}}$ & DOSE-02 & SOLID & N/A & 1 & & $\mathrm{mrad} / \mathrm{hour}$ \\
\hline 97000397 & $B Y-101$ & 8 DUP & S97T001364 & $\mathrm{OW}$ & H2ODIG01 & SOLID & 4.948 & 5.064 & N/A & $g / L$ \\
\hline 97000397 & BY-101 & 0.500 & $\rightarrow \underset{597100}{C}$ & $O_{0 \mathrm{~W}}$ & DOSE-02 & OLID & 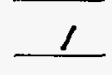 & I & U/A & hour \\
\hline
\end{tabular}

\section{Final page for worklist \# 18697}
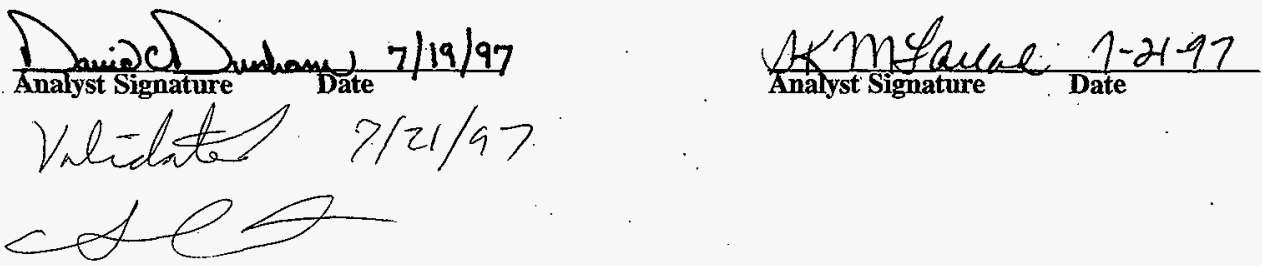

Data Entry Comments:

HPT surveyed by Joe Valdez 7/18/97.

Units shown for $Q C$ (SPK \& STD) may not reflect the actual units. $D L=$ Detection Limit, $S=$ Worklist Slot Number,

$R=$ Replicate Number, $A=$ Aliquot Code. 
HNF-SD-HM-DP-258, REV. 0

\section{BY-101 BULK DENSITY WORKSHEETS}


HNF-SD-HM-DP-258, REV. 0

\section{THIS PAGE INTENTIONALLY LEFT BLANK}


HNF-SD-WM-DP-258, REV. 0

âd.

3

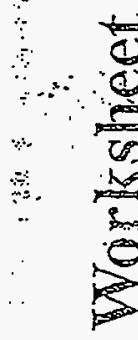

की

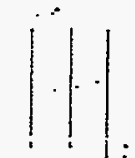

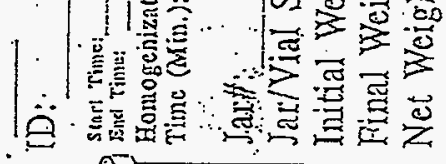

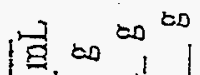

$\ddot{i} \dot{0} \stackrel{0}{0}$

से की

넝 \&
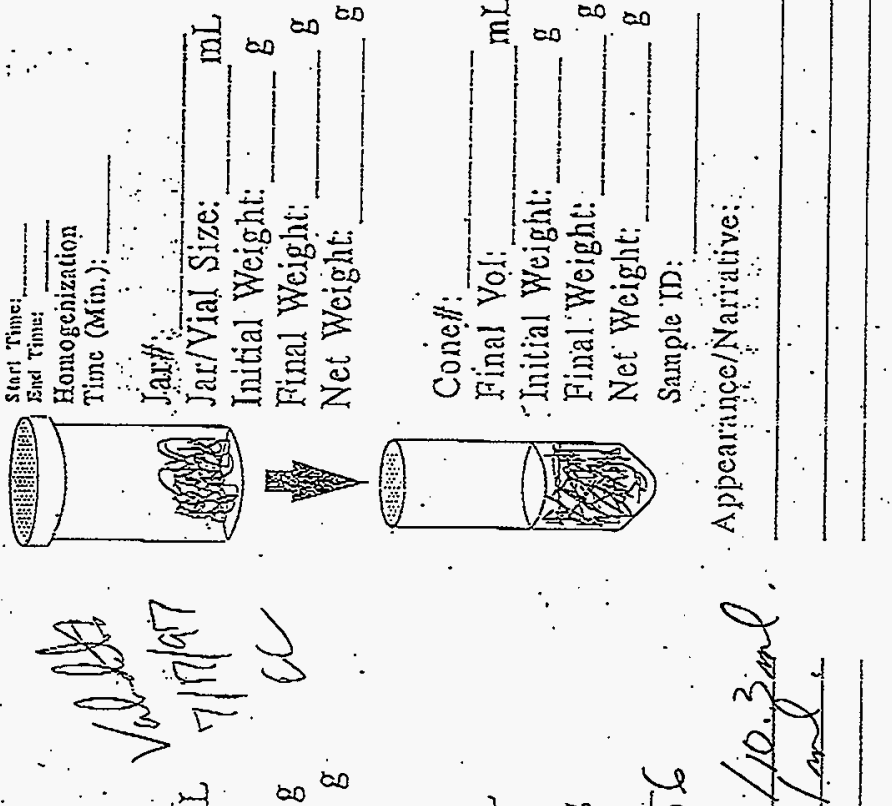

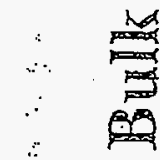
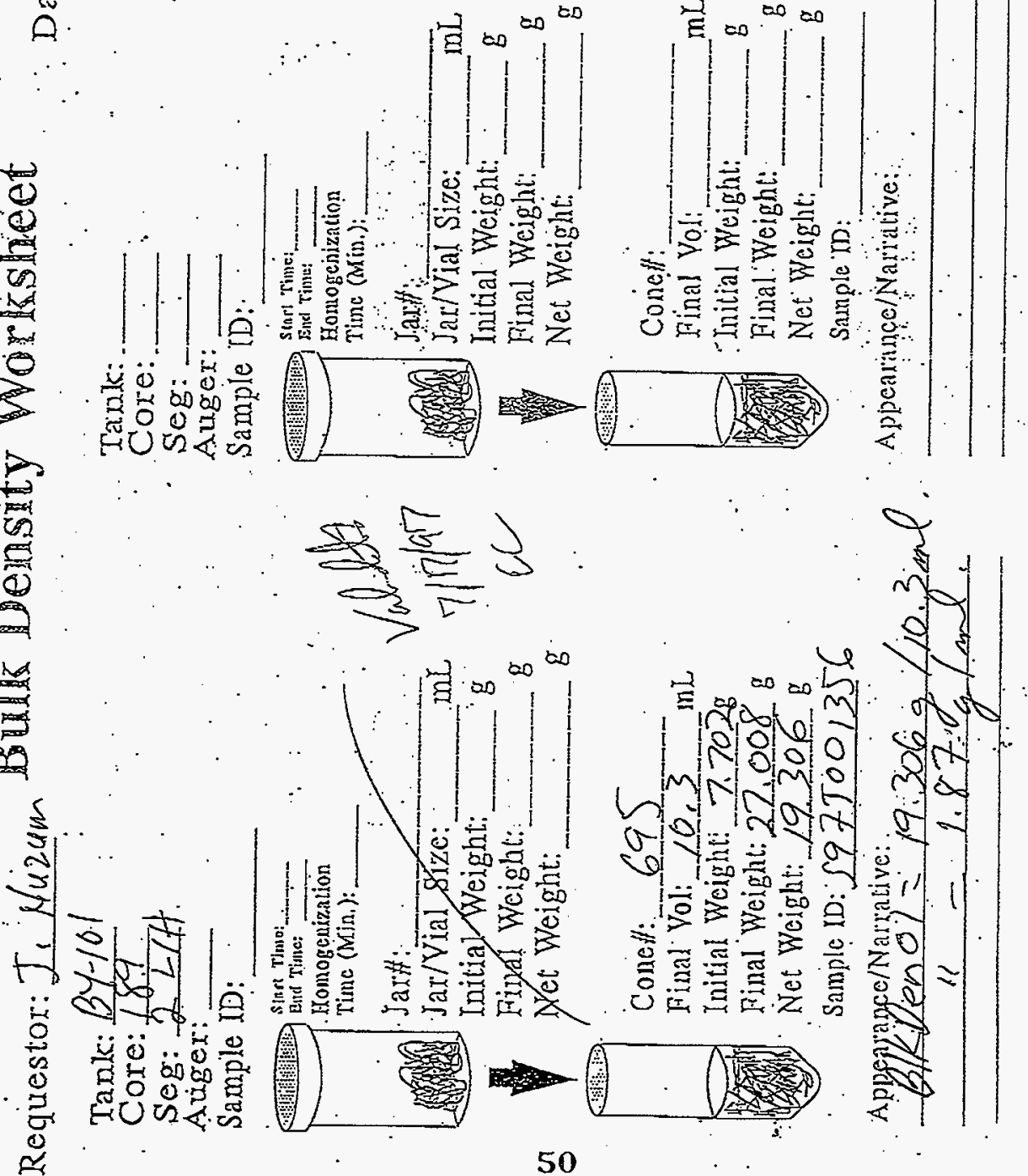

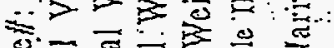

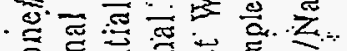

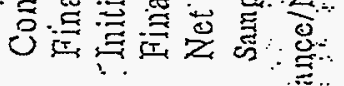




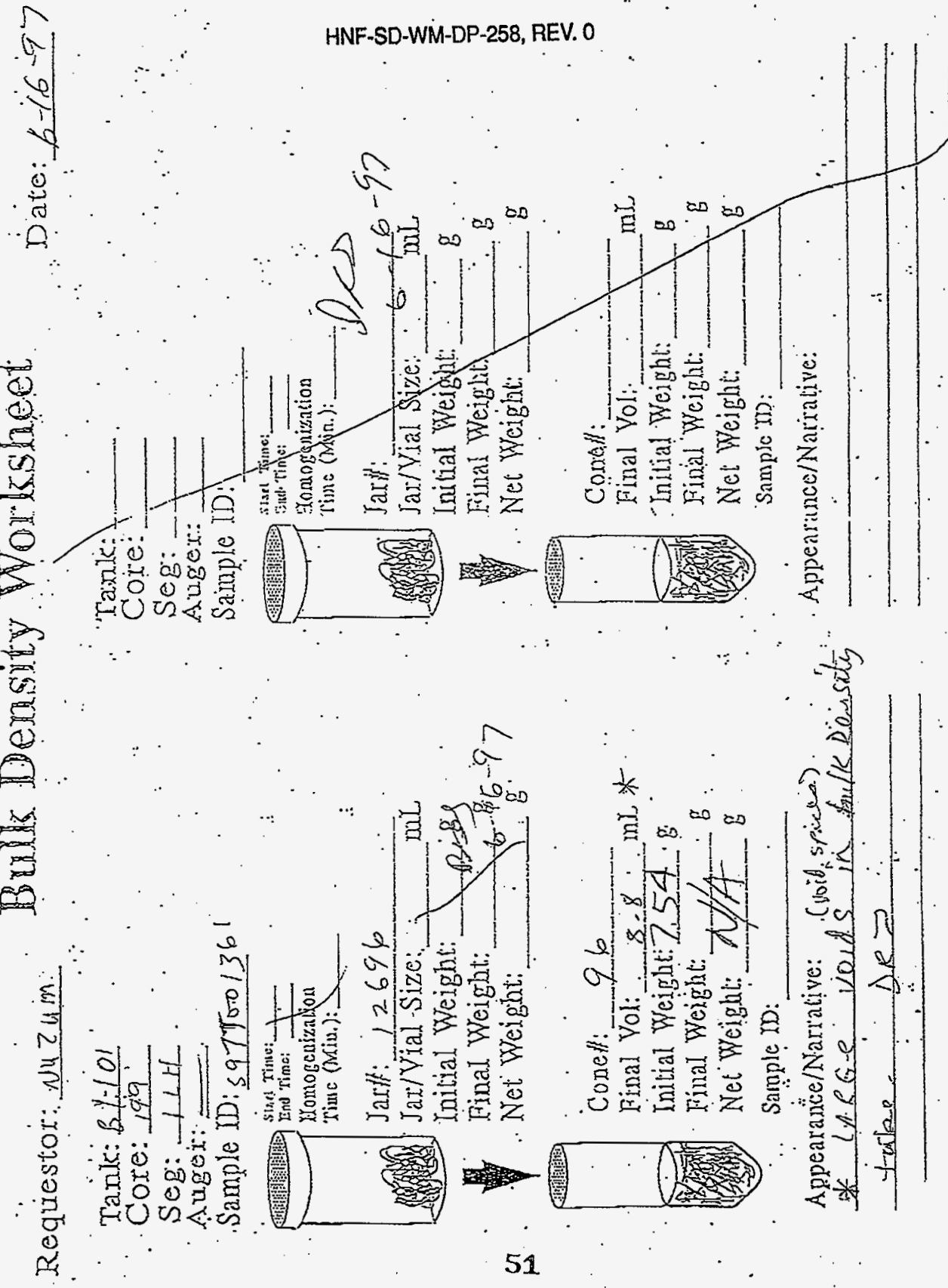


HNF-SD-WM-DP-258, REV. 0

INORGANIC ANALYSIS

52 
HNF-SD-HM-DP-258, REV, O

THIS PAGE INTENTIONALLY LEFT BLANK 
HNF-SD-WM-DP-258, REV. 0

worklistrpt Version 2.1 05/15/95

Page: 1

09/17/97 08:04

LABCORE Data Entry Template for Worklist\# 20320

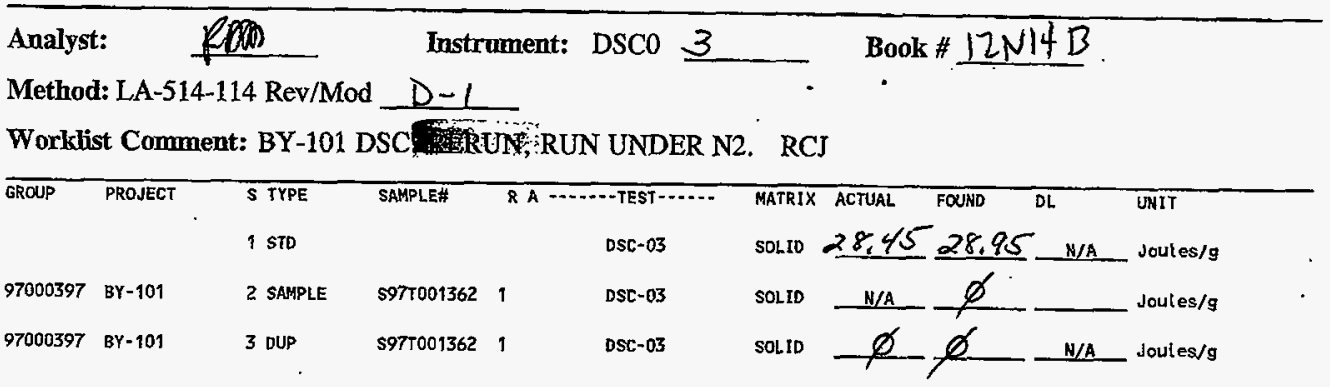

Final page for worklist \# 20320

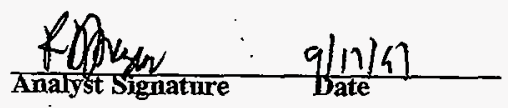

Analyst Signature Date

Data entry + validation

by 11 anatto 9-18.97

Data Entry Comments:

Units shown for $Q C$ (SPK \& STD) may not reflect the actual units. $D L=$ Detection Limit, $S=$ Worklist Slot Number, $R=$ Replicate Number, $A=$ Aliquot Code.

54 
Curve 1: DSC

Fłle info: IND091701 Wed Sep 17 19: $48: 531997$

Sample Wejght: $21.070 \mathrm{mg}$

12N14-B

SIGNATURE BELOW REPRESENTS CHEMICAL TECHNOLOGIST/CHEMIST THAT COMPLETED/VERIFIED THE CALIBRATION/ANALYSIS ON PAGES 55 TO 58 ...

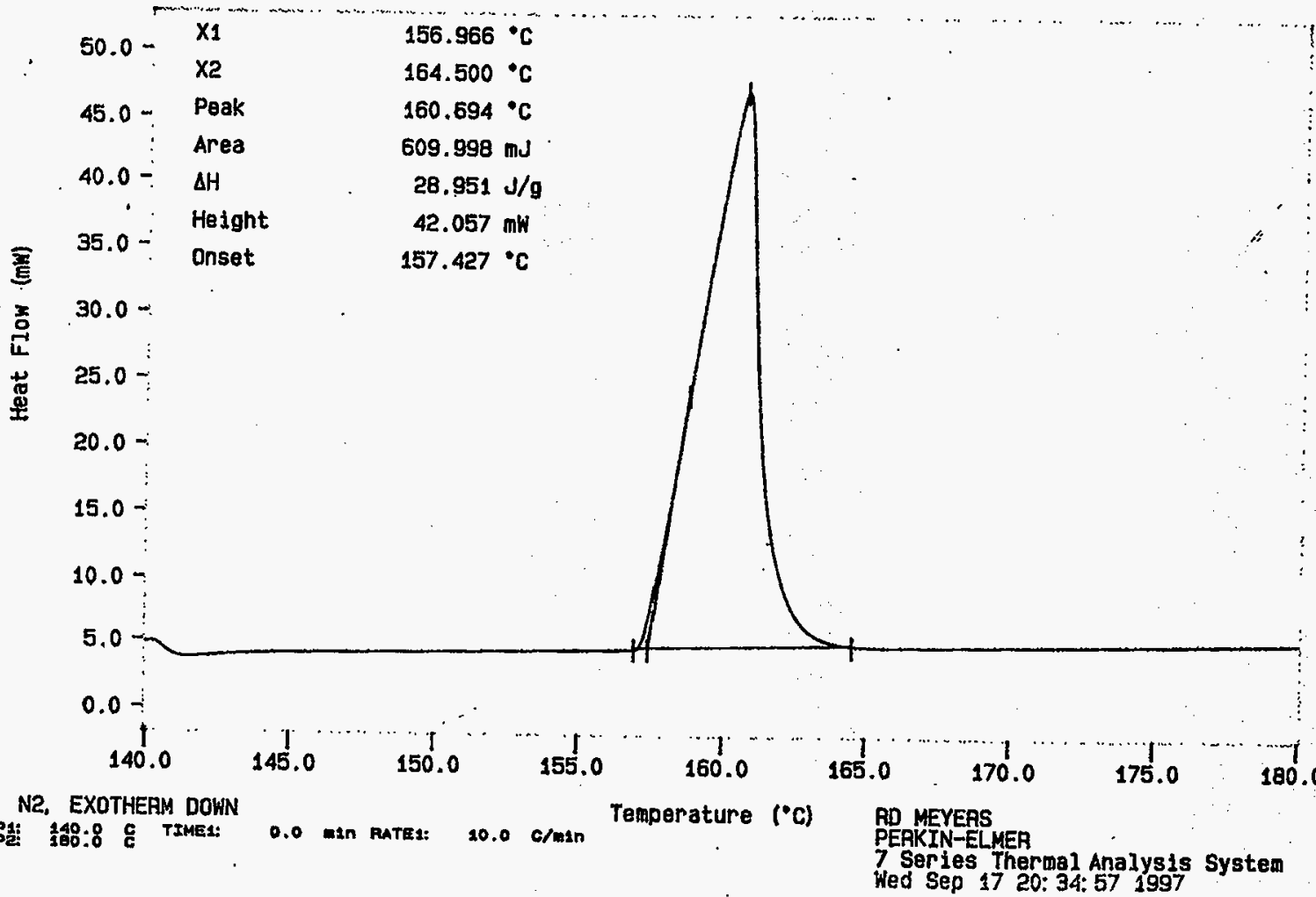

\section{IEUP: 140.0 C TIMEA:}

- min Rate1: 10.0 c/main 
HNF-SD-WM-DP-258, REV. 0

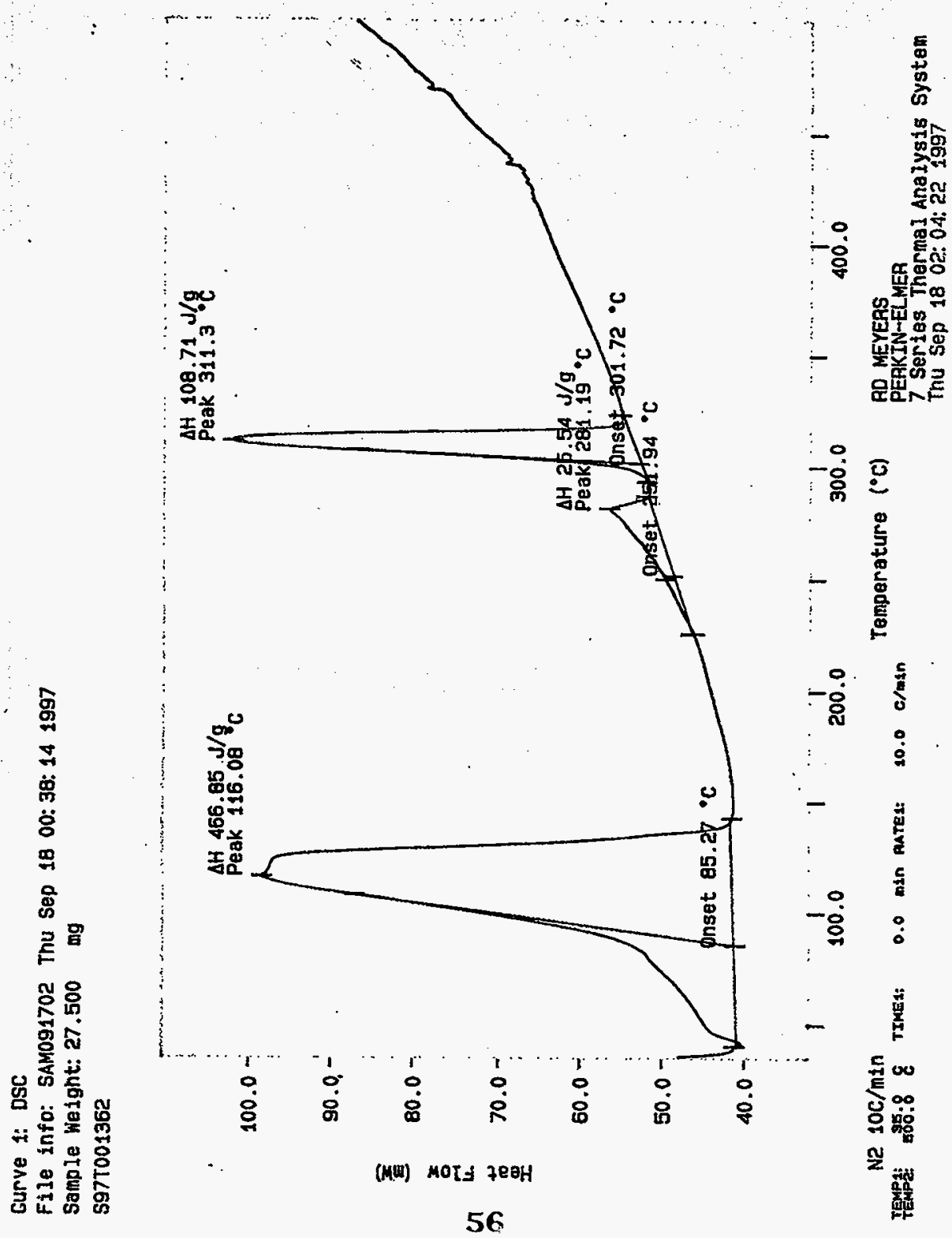


HNF-SD-WM-DP-258, REV 0

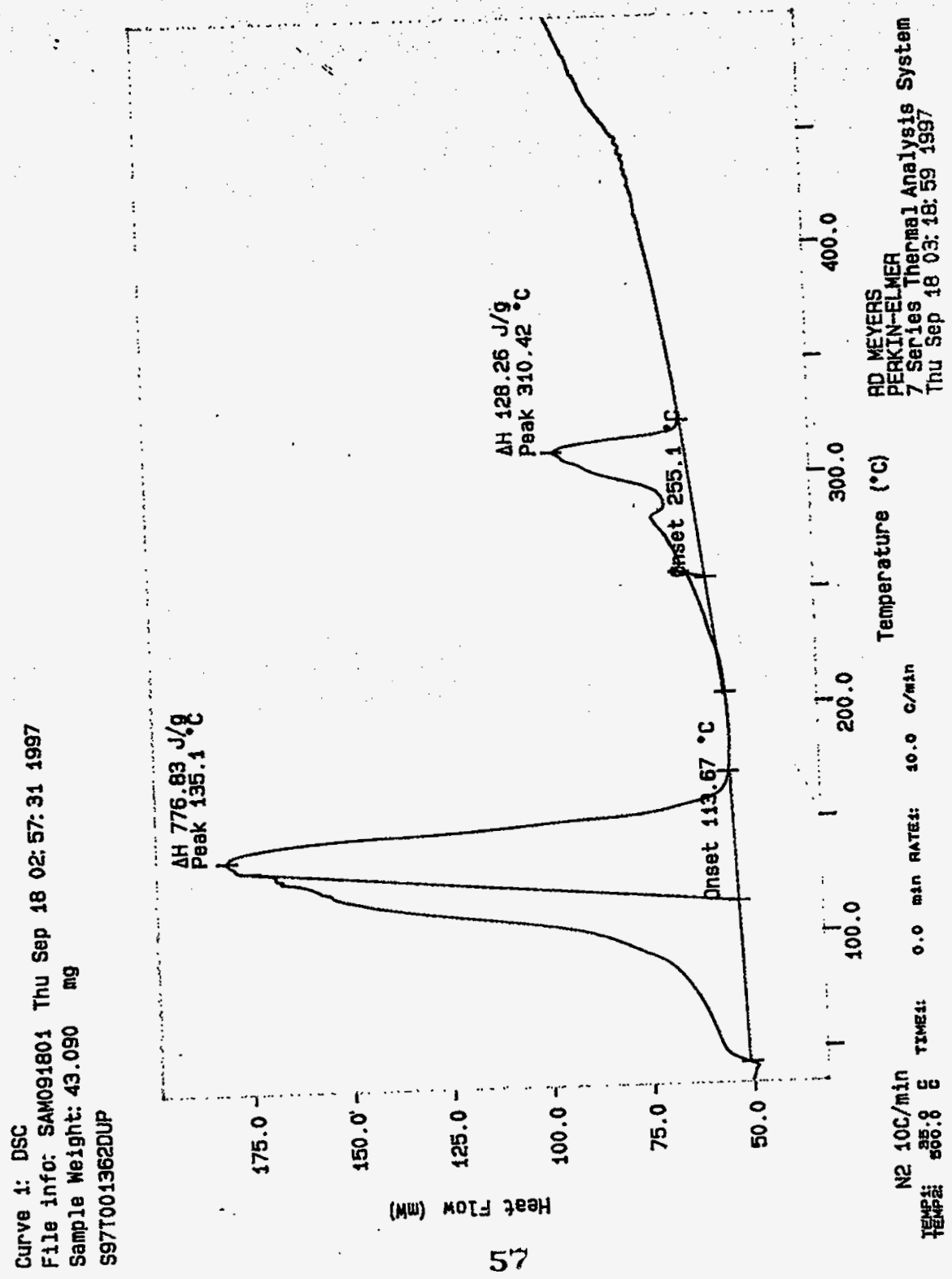


HNF-SD-WM-DP-258, REV. 0 -

THIS PAGE INTENTIONALLY LEFT BLANK

58 
worklistrpt Version $2.105 / 15 / 95$ 07/18/97 10:52

HNF-SD-WM-DP-258, REV. 0

Page: 1

\section{LABCORE Data Entry Template for Worklist\# 19383}

Analyst: STF Instrument: DSCO 3 Book I2N/4B

Method: LA-514-114 Rev/Mod D-1

Worklist Comment: BY-101 DSC, RUN UNDER N2. RCJ

\begin{tabular}{|c|c|c|c|c|c|c|c|c|c|c|}
\hline GROUP & PROJECT & $\begin{array}{l}\text { S TYPE } \\
1 \text { STO }\end{array}$ & SAMPLE\# & RA & DSC -03 & $\begin{array}{l}\text { MATRIX } \\
\text { LIQUID }\end{array}$ & $\begin{array}{l}\text { ACTUAL } \\
28.45 \\
\end{array}$ & $\begin{array}{l}\text { FOUND } \\
\text { D8. } 28 \%\end{array}$ & $\begin{array}{l}\mathrm{DL} \\
\mathrm{N} / \mathrm{A}\end{array}$ & $\begin{array}{l}\text { UNIT } \\
\text { Joules/g }\end{array}$ \\
\hline 97000397 & BY-101 & 2 SAMPLE & s97T001365 & 0 & DSC-03 & LIQUID & $N / A$ & 0 & & Joules/g \\
\hline 97000397 & BY-101 & 3 DUP & s97T001365 & 0 & DSC-03 & LIQUID & 0 & 0 & N/A & Joules/g \\
\hline
\end{tabular}

Final page for worklist \# 19383

Aralyst Signature Date

tultin $7-18-97$

Validated $7 / 22 / 97$ hachelor

Analyst Signature

$7 / 22 / 97$

Data Entry Comments:

Units shown for $Q C$ (SPK \& STD) may not reflect the actual units. $D L=$ Detection Limit, $S=$ Worklist Slot Number, $R=$ Replicate Number, $A=$ Aliquot Code. 
Curve 1: nSC

F118 info: IND071801 FrI Ju1 18 08: 26: 221997

Sample velght: 24.590 ag 12N14-B

SIGNATURE BELOW REPRESENTS CHEMICAL TECHNOLOGIST/CHEMIST THAT COMPLETEDNERIFIED THE CALIBRATION/ANALYSIS ON PAGES 600 TO 62.

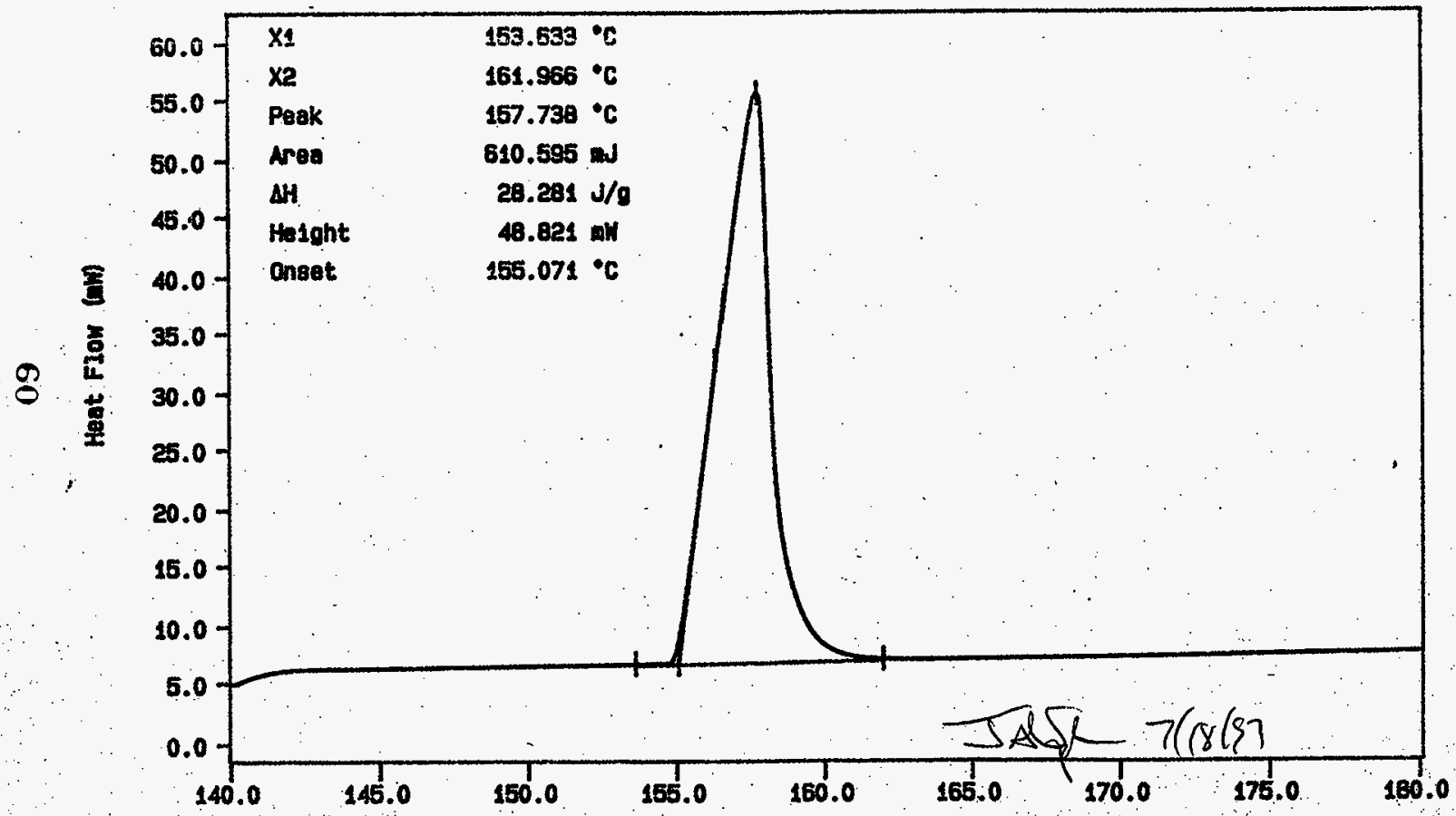

Ne. EXOTHEFN DOWN Ra: $148: 8 \cdot g$ rmat: 
Curve 1: DSC

F1le info: SAM071814 Sat JuI 19 00:08: 331997

Sample Weight: $10.070 \mathrm{ag}$

S97T001365

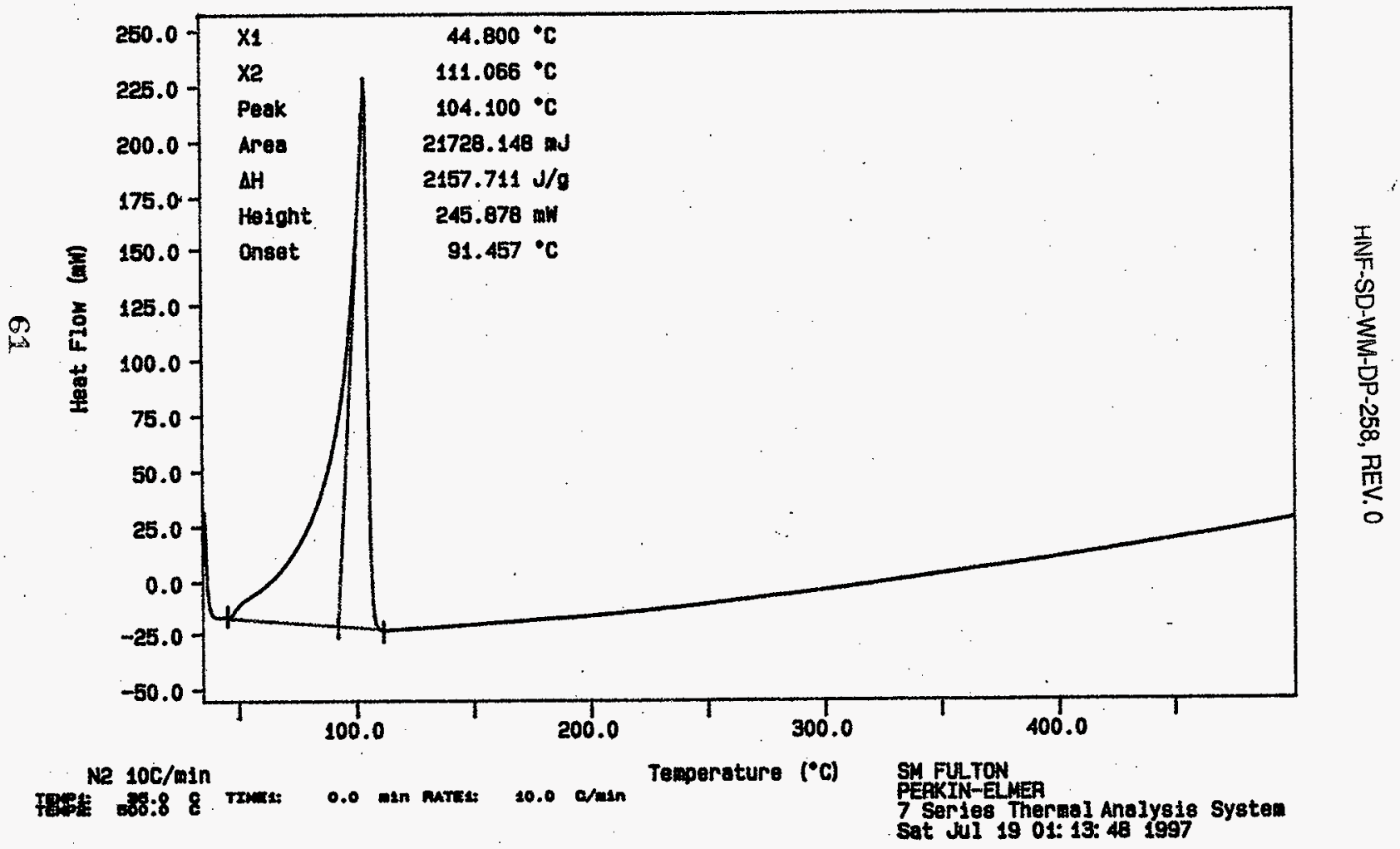


Curve 1: DSC

F11e info: SAw071815 sat Jul 19 02:03:23 1997

Sanple Weight: 10.090

ng

S97T001365 DUP

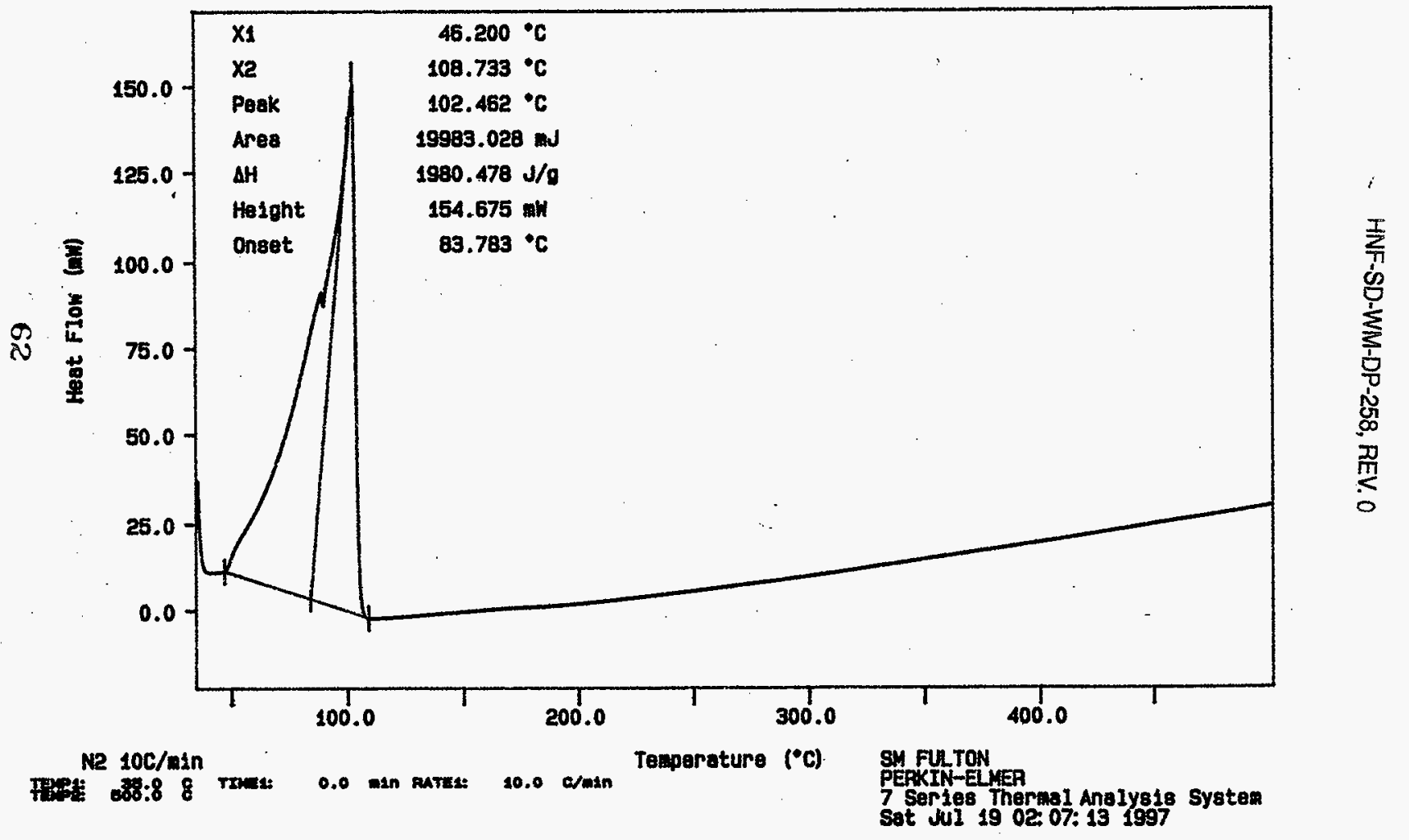




\section{LABCORE Data Entry Template for Worklist\# 19384}

Analyst: $\quad$ SMF Instrument: $D S C 0$ 3 Book 12 N14 $-B$

Method: LA-514-114 Rev/Mod D-1

Worklist Comment: BY-101 DSC, RUN UNDER N2. RCJ

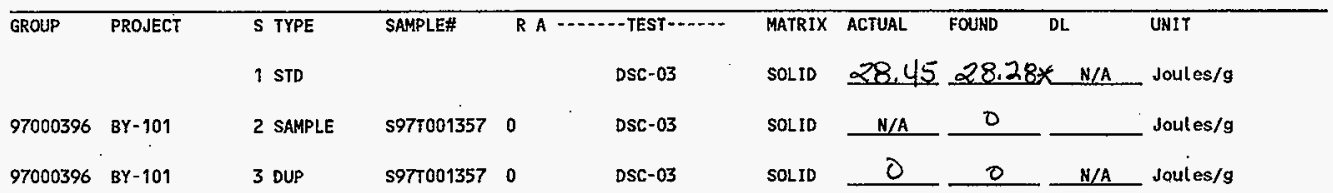

\section{Final page for worklist \# 19384}

\section{Analyst Signature Date}

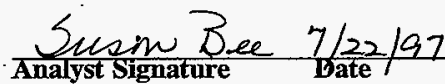

Validated H122/97 Q8hachelor

Units shown for $Q C$ (SPK \& STD) may not reflect the actual units. $D L=$ Detection Limit, $S=$ Worklist Slot Number, $R=$ Replicate Number, $A=$ Aliquot Code. 
Curve 1: DSC

File info: InD071801 Fri لlul 18 06: 26: 221997

Sample Waight: 24.590 mg

12N14-B

SIGNATURE BELOW REPRESENTS CHEMICAL TECHNOLOGIST/CHEMIST THAT COMPLETEDIVERIFIED THE CALIBRATIONIANALYSIS ON PAGES $/ 14$ TO $6 \mathrm{CC}$.

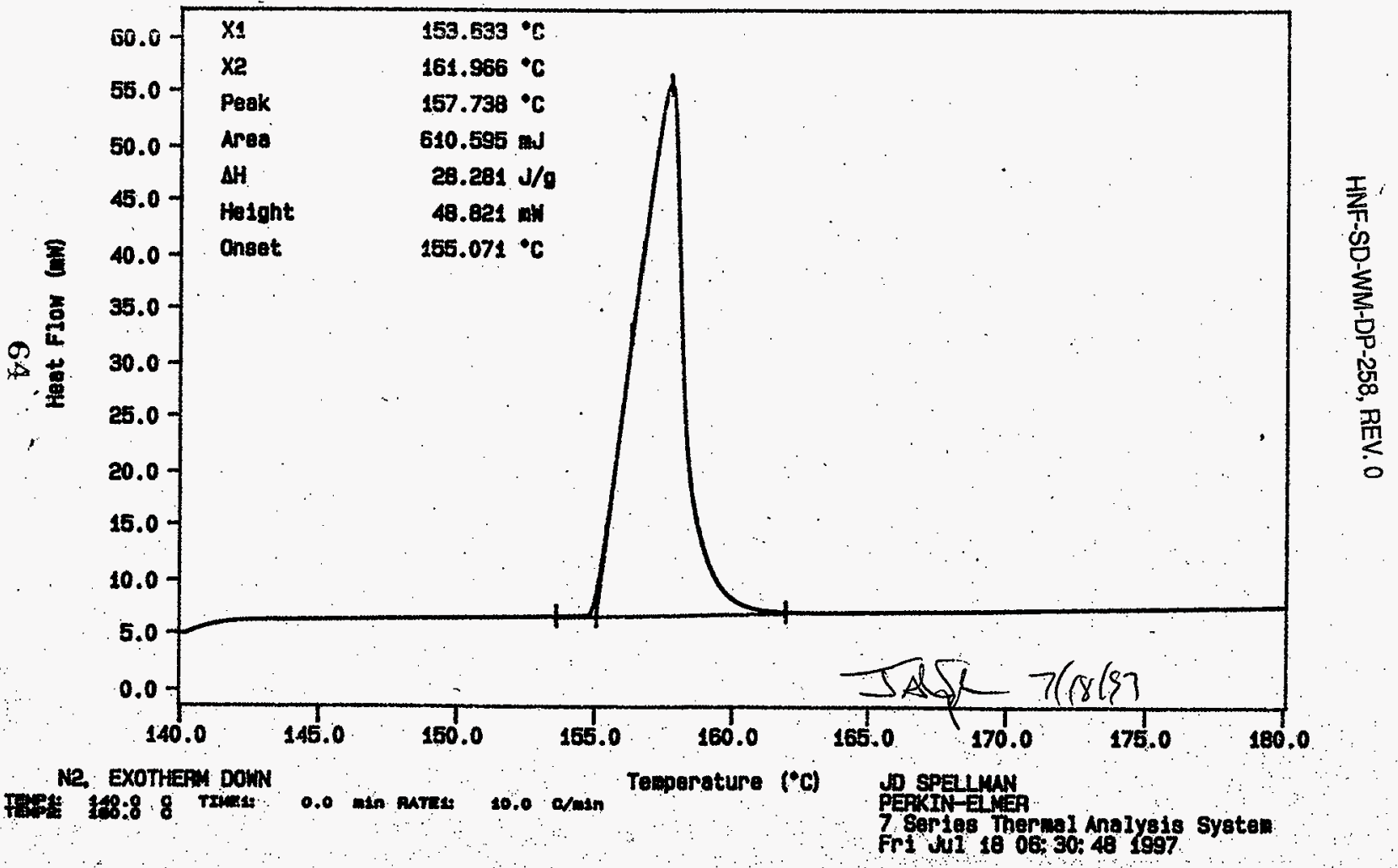


Curve 1: DSC

File info: SAN071812 Sat Jul 19 02 56: 391997

Sample Meight: 31.360

s97T001357

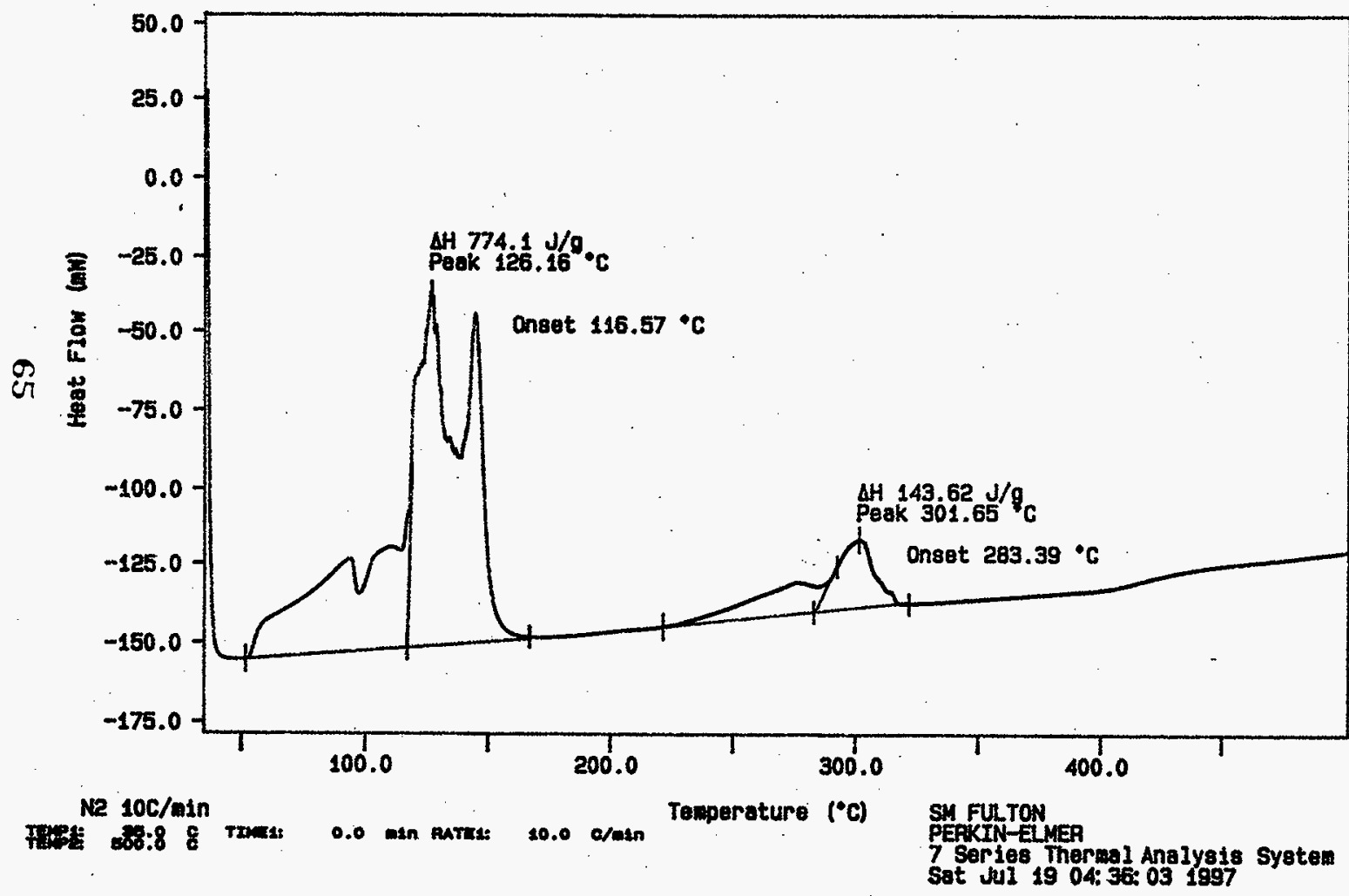


Curve 1: DSC

File info: SAM071813 Sat Jul 19 05: 25: 481997

Sample Weight: 13.940

5977001357 DUP

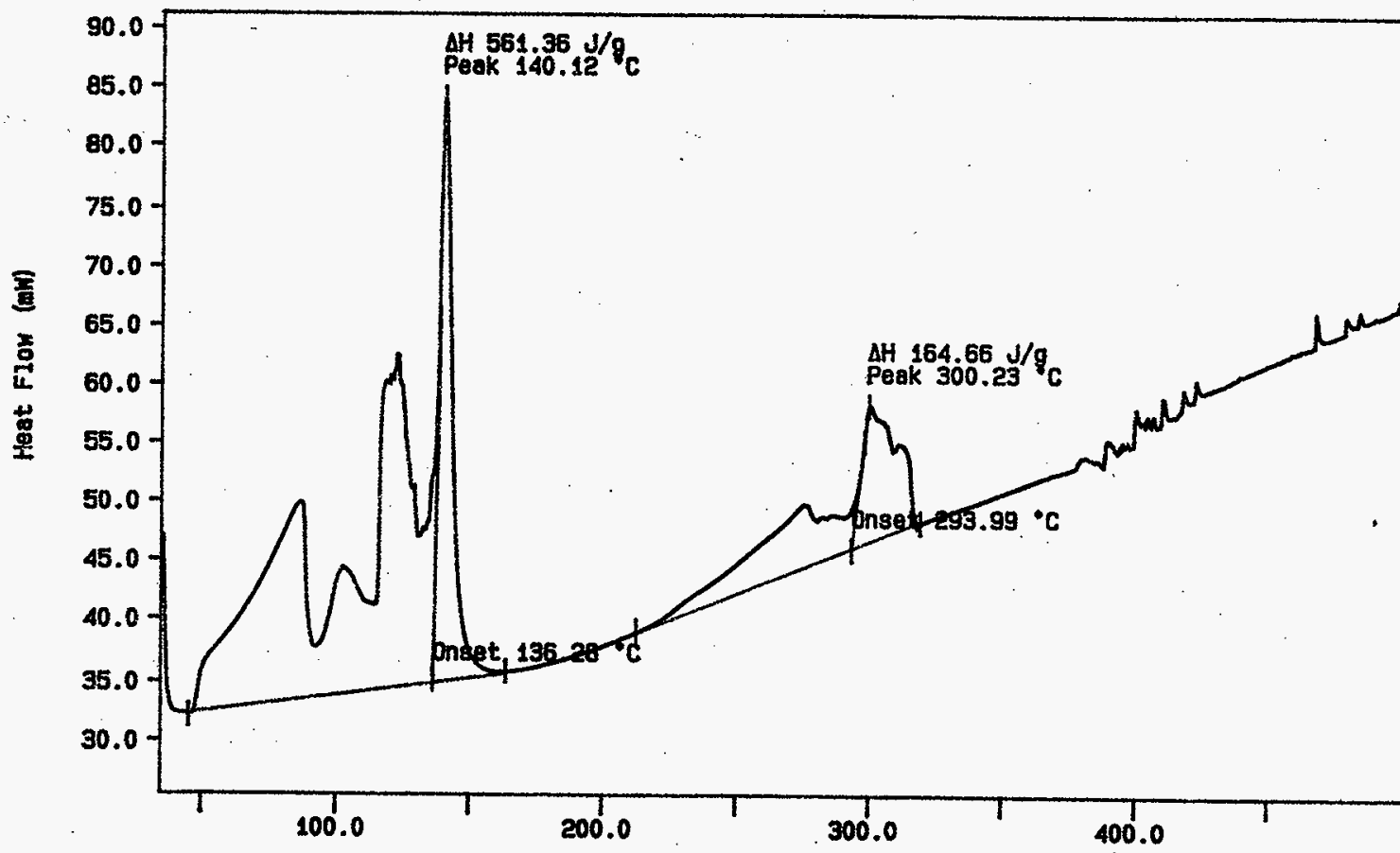

N2 10c/min Fept: $8.80: 8$ \& Trmat:

0.0 man ratast $10.0 \mathrm{c} / \mathrm{man}$

Temperature $\left({ }^{\circ} \mathrm{C}\right)$

Sy FULTON

PERKIN-ELMER

7 Series Theraal Analyeis System

Sat Jul 19 06: 49;08 1997 


\section{LABCORE Data Entry Template for Worklist\# 19431}

Page: $\quad 1$

\begin{tabular}{|c|c|c|c|}
\hline Analyst: & oolo & Instrument: & DSC0 \\
\hline
\end{tabular}

Method: LA-514-113 ReAlate y/22/97

Worklist Comment: DSC-02 BY-101 liquid. -ppb

\begin{tabular}{|c|c|c|c|c|c|c|c|c|c|c|}
\hline$\overline{\text { GROUP }}$ & PROJECT & S TYPE & SAMPLE\# & & - TEST $\cdots . . . .$. & MATRIX & ACTUAL & FOUND & DL & UNIT \\
\hline 97000397 & $B Y-101$ & 1 SAMPLE & S97T001365 & 0 & DSC-02 & LIQUID & $N / A$ & 0 & & Joules/g D \\
\hline 97000397 & $B Y-101$ & 2 DUP & \$97T001365 & 0 & DSC-02 & LIQUTD & 0 & 0 & & Joul \\
\hline
\end{tabular}

\section{Final page for worklist \# 19431}

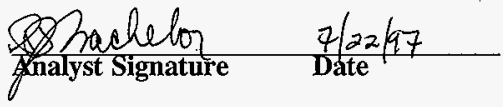

Analyst Signature Date

Data Entry Comments:

Units shown for $Q C$ (SPK \& STD) may not reflect the actual units. $D L=$ Detection Limit, $S=$ Worklist Slot Number, $R=$ Replicate Number, $A=$ Aliquot Code. 


\section{LABCORE Data Entry Template for Worklist\# 19432}

\section{Analyst:

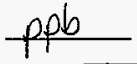 \\ Instrument: $\mathrm{DSCO} 2$ \\ Book \# NA}

Method: $\mathrm{AA}-514-113 \mathrm{Rev} / \mathrm{Mod}$ Z/2a/fy

Worklist Comment: DSC-02 BY-101 solid. -ppb

\begin{tabular}{|c|c|c|c|c|c|c|c|c|c|c|}
\hline GROUP & PROJECT & S TYPE & SAMPLE\# & $\mathbf{R}$ & - TEST----- & MATRIX & ACTUAL & FOUND & $\mathrm{DL}$ & UNIT \\
\hline 97000396 & BY-101 & 1 SAMPLE & S97T001357 & 0 & DSC- 02 & SOLID & $\mathrm{N} / \mathrm{A}$ & 0 & & Joules/g Dry \\
\hline 97000396 & BY- 101 & 2 DUP & S97T001357 & 0 & DSC-02 & SOLID & $\theta$ & 0 & $\mathrm{~N} / \mathrm{A}$ & Joules/g Dry \\
\hline 97000397 & BY -101 & 3 SAMPLE & S97T001362 & 0 & DSC-02 & SOLID & N/A & 54.70 & & Joules/g Dry \\
\hline 97000397 & BY -101 & 4 DUP & \$97r001362 & 0 & DSC-02 & SOLID & 54.70 & 0 & $N / A$ & Joules/g Dry \\
\hline
\end{tabular}

\section{Final page for worklist \# 19432}

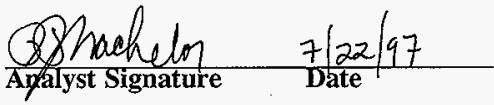

\section{Analyst Signature Date}

Data Entry Comments:

Units shown for QC (SPK \& STD) may not reflect the actual units. $D L=$ Detection Limit, $S=$ Worklist Slot Number, $R=$ Replicate Number, $A=$ Aliquot Code. 


$$
H N F-S D-2 N M-D P-258 \text {, Rev. } 0
$$

worklistrpt Version $2.105 / 15 / 95$

Page: $\quad l$ 09/19/9708:21

LABCORE Data Entry Template for Worklist\# 20371

Analyst: Ha Instrument: $\operatorname{DSC} 3$ Book \#)

Method: LA-514-113 Rev/Mod

Worklist Comment: BY-101 Dry DSC

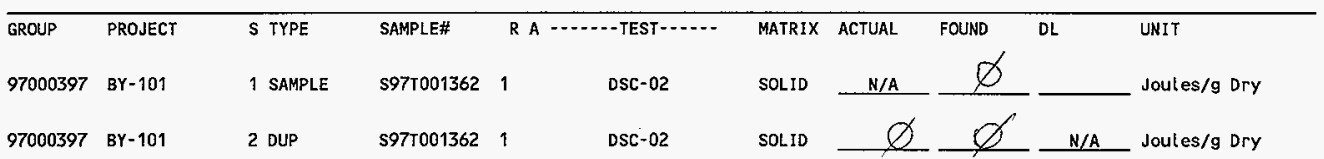

Final page for worklist \# 20371

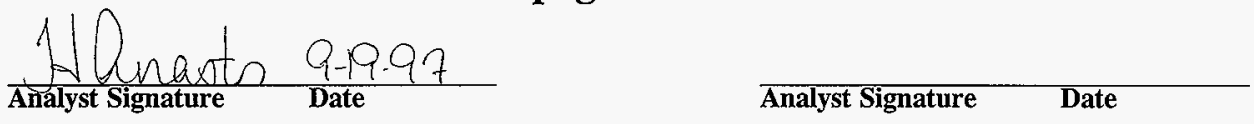

Iata curry + validation Ila 9-19-97

Wet $D S C=\varnothing \frac{\text { SAm }}{\varnothing}$ Dup

Data Entry Comments:

Units shown for $Q C$ (SPK \& STD) may not reflect the actual units. $D L=$ Detection Limit, $S=$ Worklist Slot Number, $R=$ Replicate Number, $A=$ Aliquot Code.

68.1 


\section{LABCORE Data Entry Template for Worklist\# 18781}

Analyst: $\quad$ DPB Instrument: TGA0 3 Book \# 103N8A

Method: LA-514-114 Rev/Mod D - O

Worklist Comment: BX110, BY101) TGA-03 Run under Nitrogen. skm

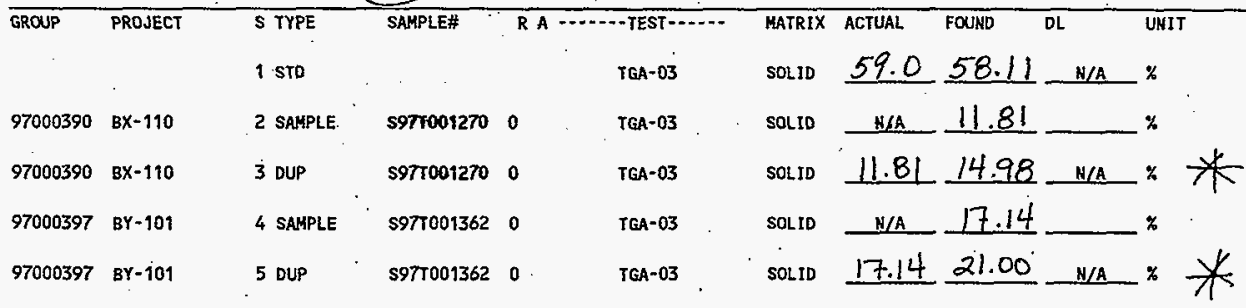

\section{Final page for worklist \# 18781}

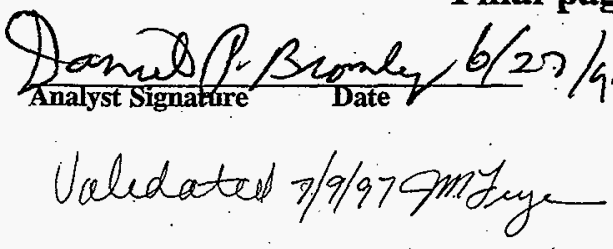

* QC exceeds UCL

Data Entry Comments:

Units shown for $Q C$ (SPK \& STD) moy not reflect the actual units. $D L=$ Detection limit, $S=$ Workiist Slot Number, $R=$ Replicate Number, $A=$ Aliquot Code. 
Curve 1: TGA

File info: TER06270 $\mathrm{FrI}$ Jun 27 06: 23: 211997

Sample Weight: $27.279 \mathrm{mg}$

STD 103NB-A

* I STD 103N8 A: TEROG2701

$x$ weight (wt. $x$ )

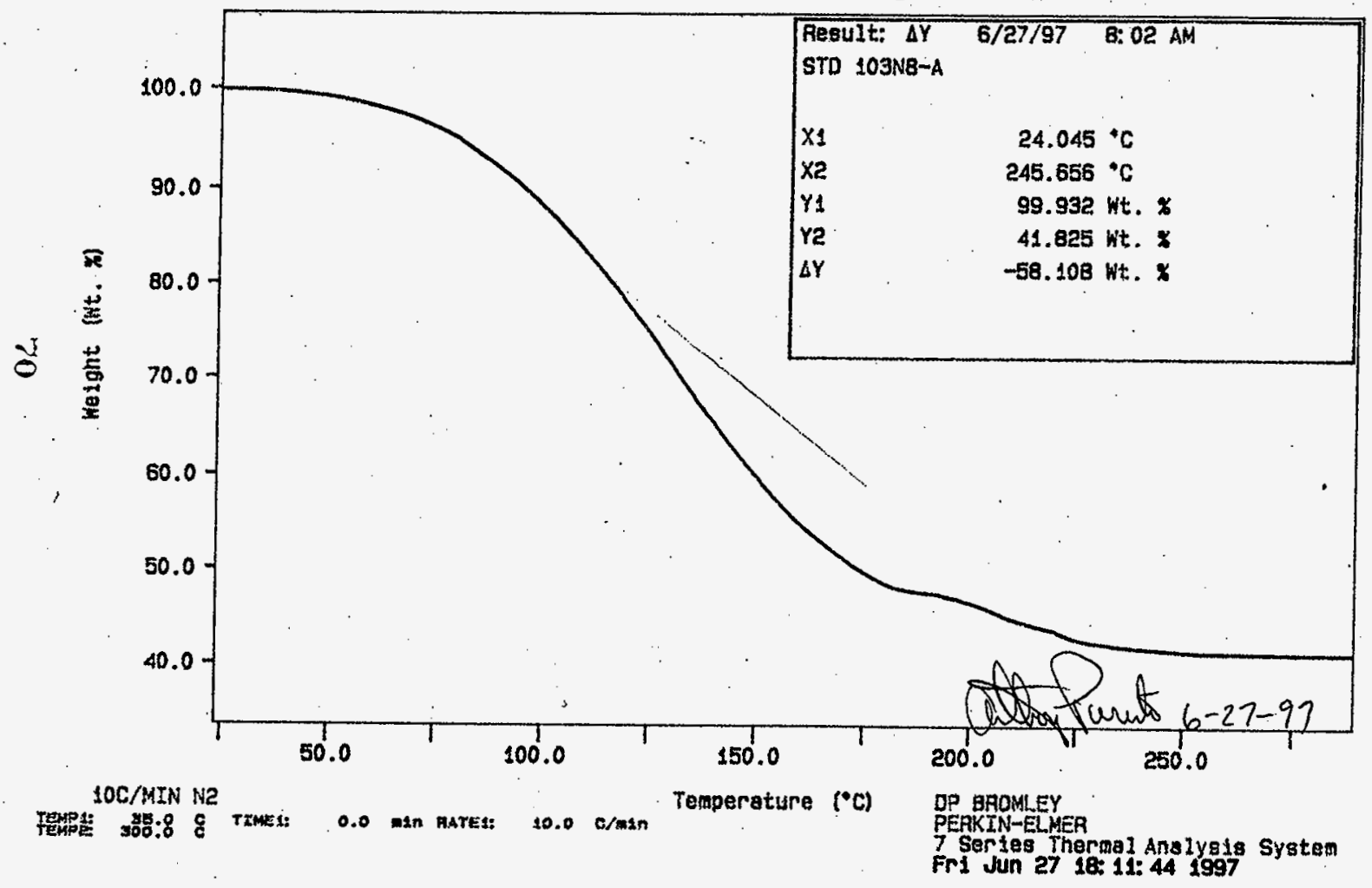


Curve 1: TGA

F110 info: SAM062703 Frt Jun 27 13:17: 131997

Sample Height: 13.248

S97TO01270SAM

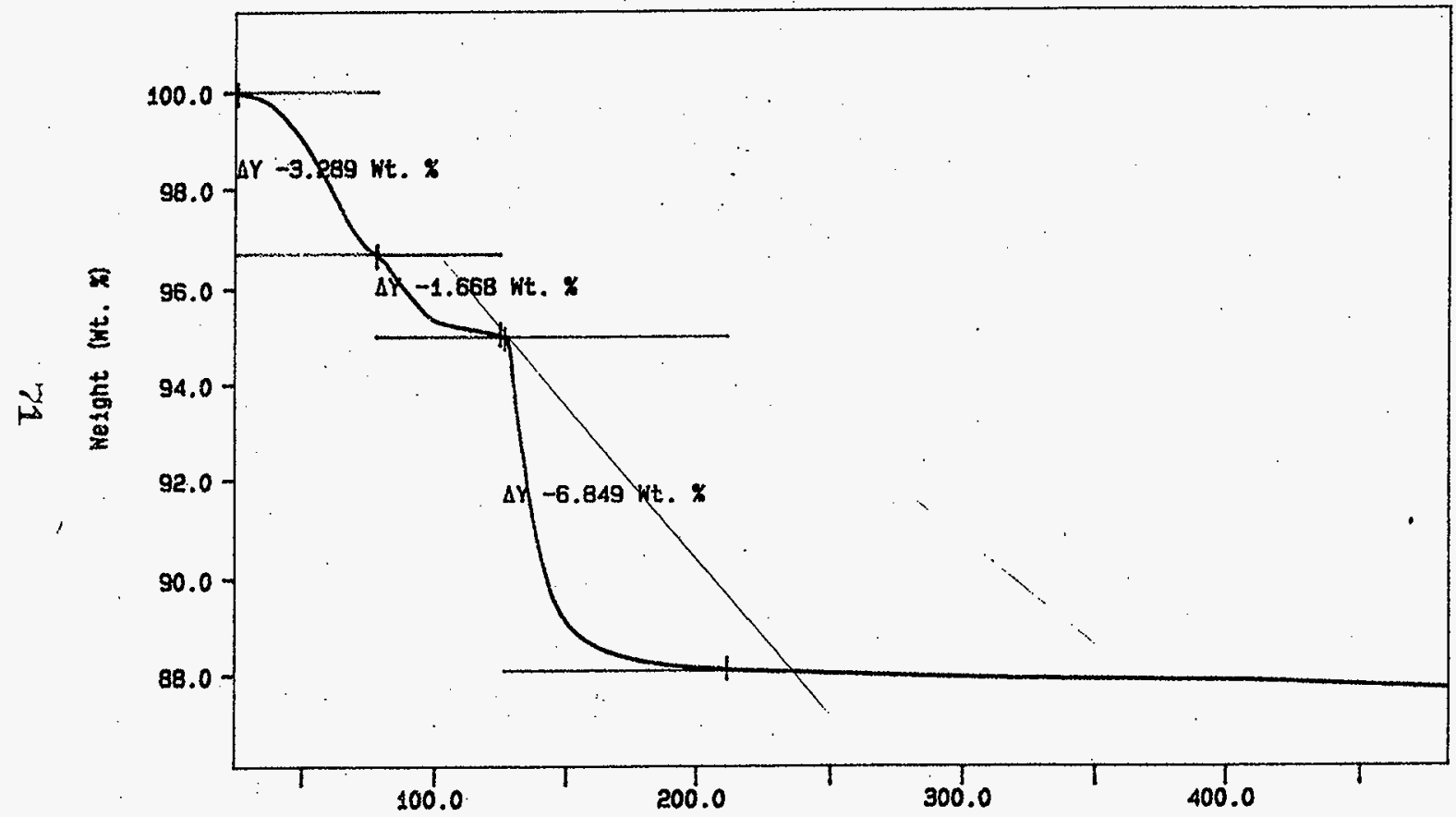

10C/MIN N2

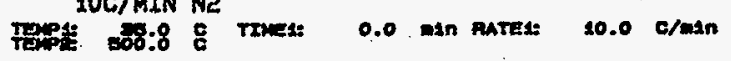
Temperature $\left(^{\circ} \mathrm{C}\right)$ DP BROMLEY PERKIN-ELMEA 
Curve 1: TGA

F1le info: SAM062704 Frl Jun 27 14: 28: 311997

Sample Height: 9.128

ag

S97TO01270DUP
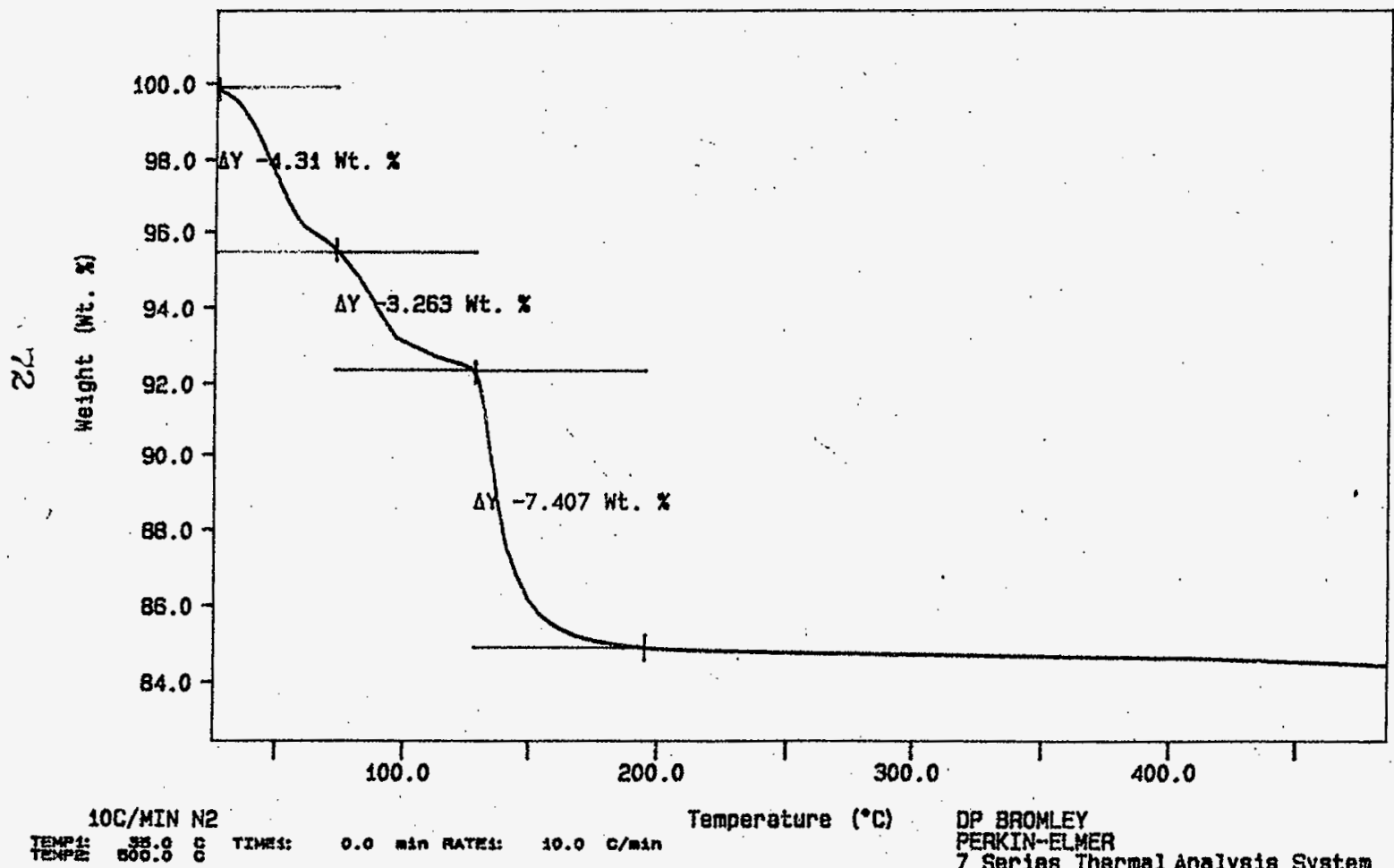

Temperature ( $\mathrm{C})$

DP BROMLEY

PERKIN-ELYEA

7 Serjes Therms Analysts System Frí Jun 27 15: 32381997 
Curve 1: TGA

File info: SAM062705 Fri Jun 27 18:52: 241997

Sample Weight: $18.733 \mathrm{mg}$

S97T001362 SAM

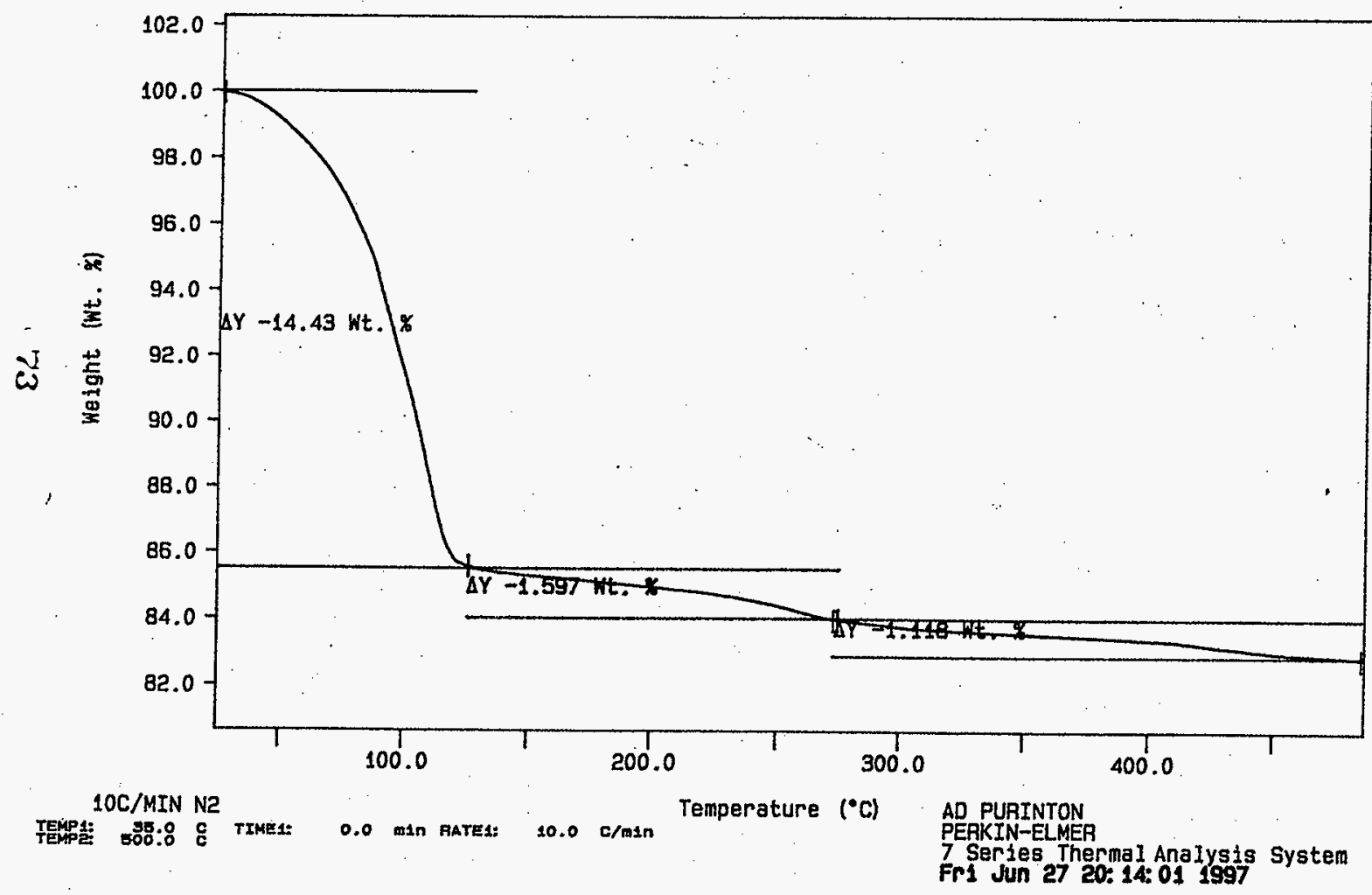


Curve 1: TGA

F1le info: SAM062706 Fri Jun 27 21:11: 381997

Sample Weight: 16.876

S97T001362 DUP

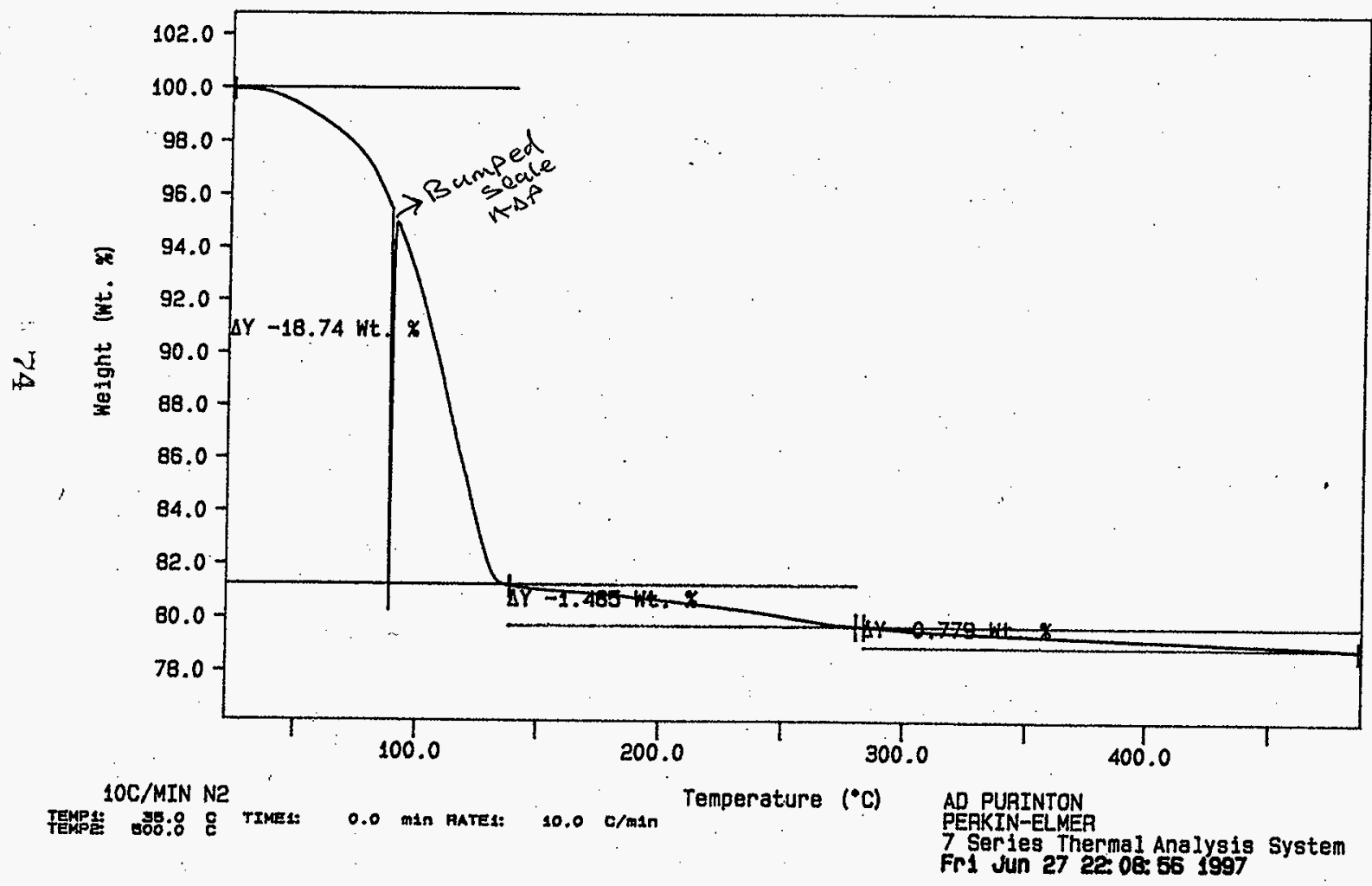




\section{LABCORE Data Entry Template for Worklist\# 19381}

Analyst: $\frac{\text { SMF }}{\text { Instrument: TGAO } 3}$ Book $103 \mathrm{~N} 8-\mathrm{A}$

Worklist Comment: BY-101 TGA, RUN UNDER N2. RCJ

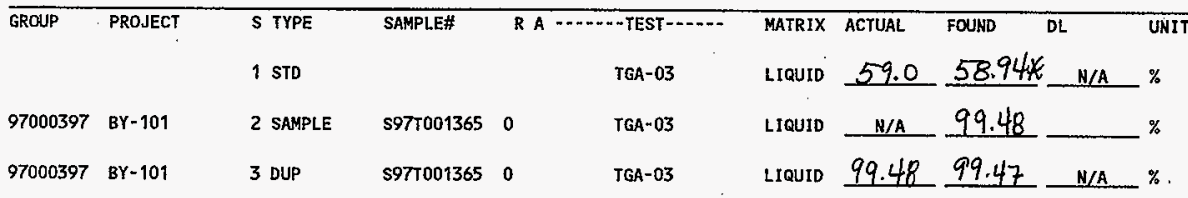

Final page for worklist \# 19381

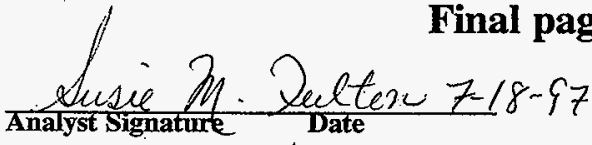

$$
\text { vidated 7/22/97 Boucher }
$$

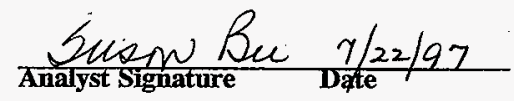

Data Entry Comments:

Units shown for $Q C$ (SPK \& STD) may not reflect the actual units. $D L=$ Detection Limit, $\dot{S}=$ Worklist Slot Number, $R=$ Replicate Number, $A=$ Aliquot Code. 
Curve 1: TEA

F11e Info: TERO71801 Fri Jul 18 06: 47:53 1997

Sauple Height: 13.855 an

STD 103n's-A

SIGNATURE BELOW REPRESENTS CHEMICAL TECHNOLOGISTICHEMIST THAT

COMPLEFED/NERIFIED THE CALIBRATION/ANALYSIS ON PAGES 76 TO 18

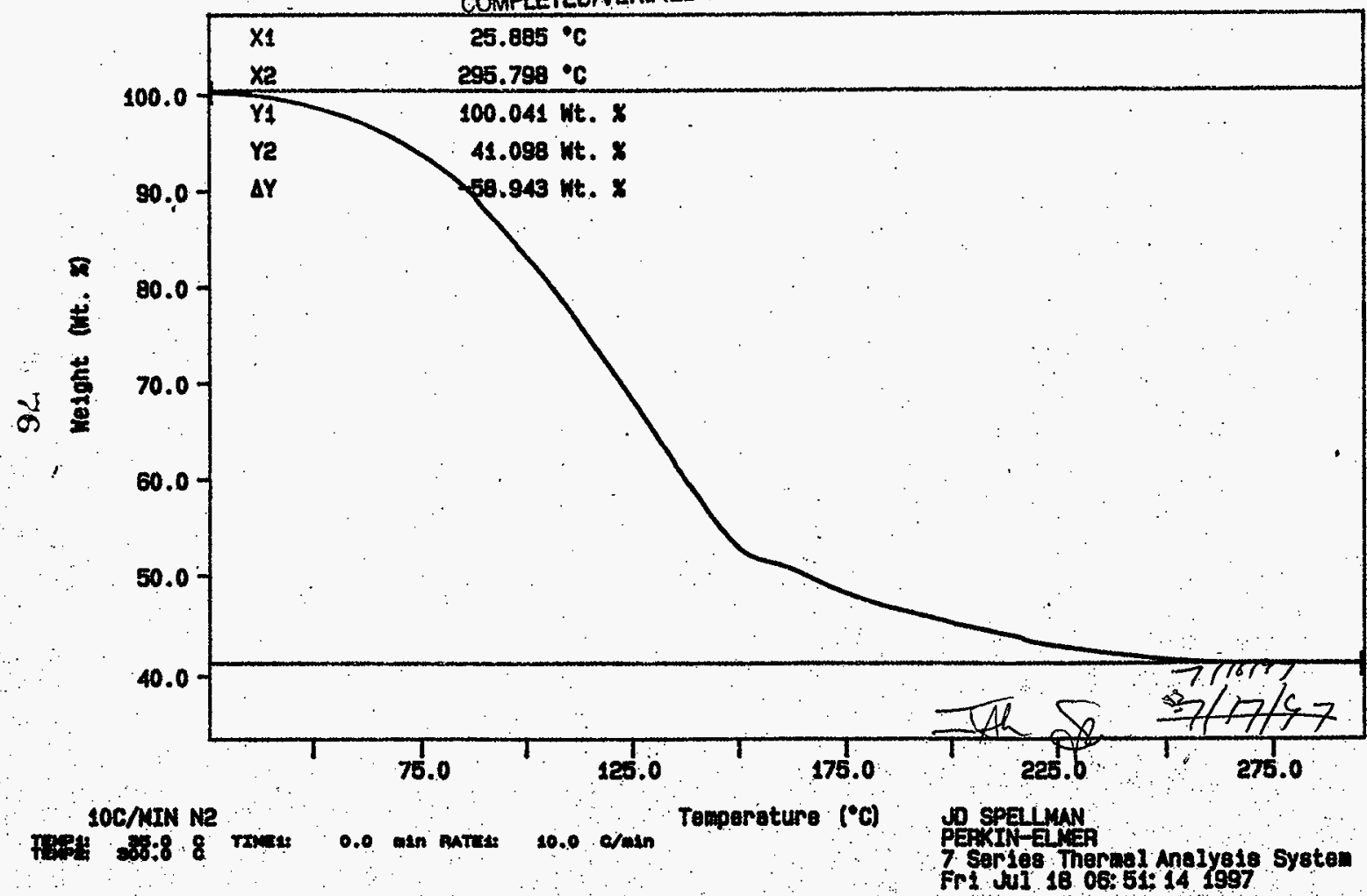


Curve 1: TEA

F11e info: SAM071814 Frl wil 18 23: $13: 431997$

Sample Weight: 10.410 ag

S97T001355

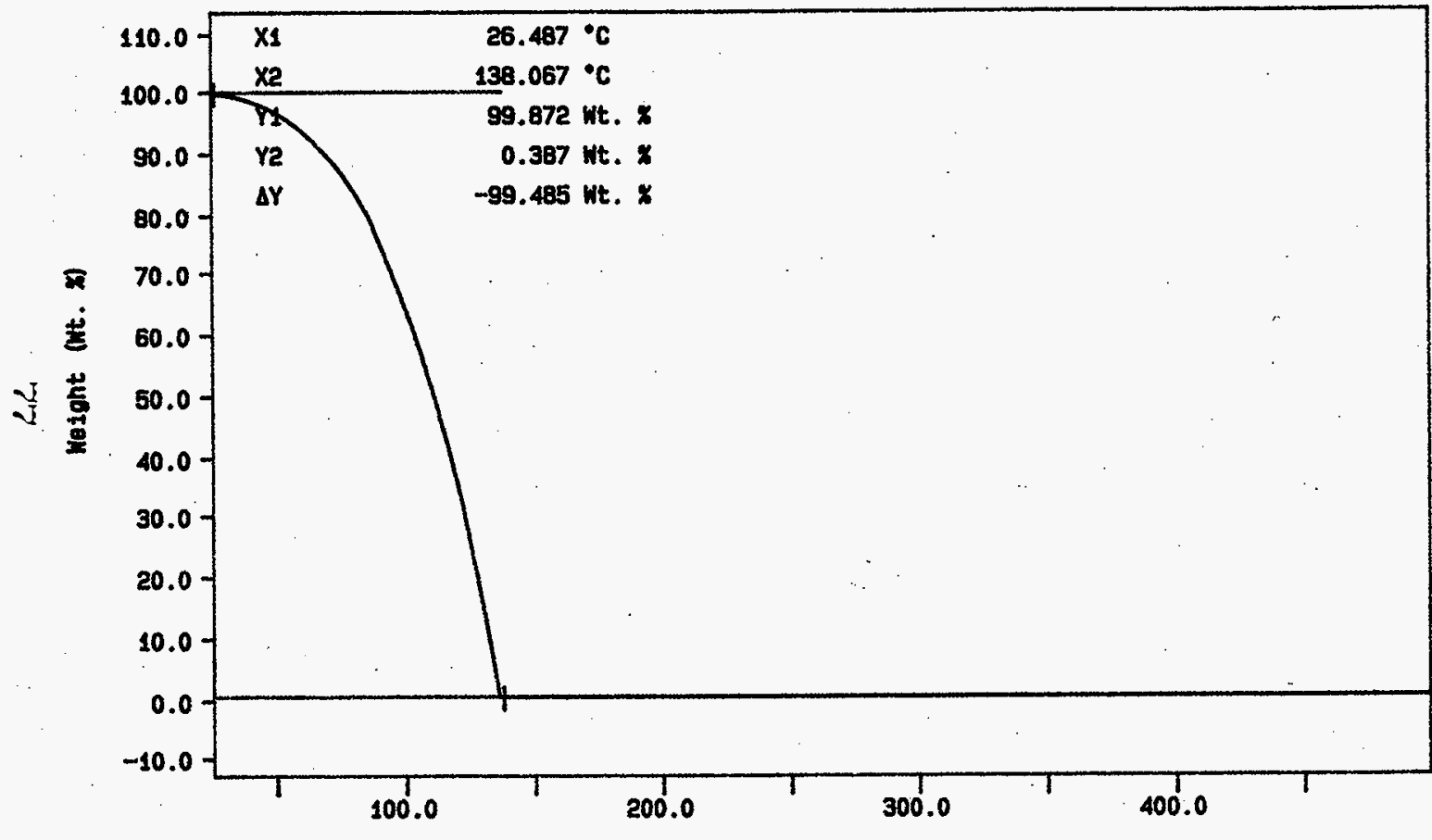


Curve 1: T6A

F11e info: SAM071815 Sat wul 19 00:35:00 1997

Sample Weight: 10.161

$597 T 001365$ DuP

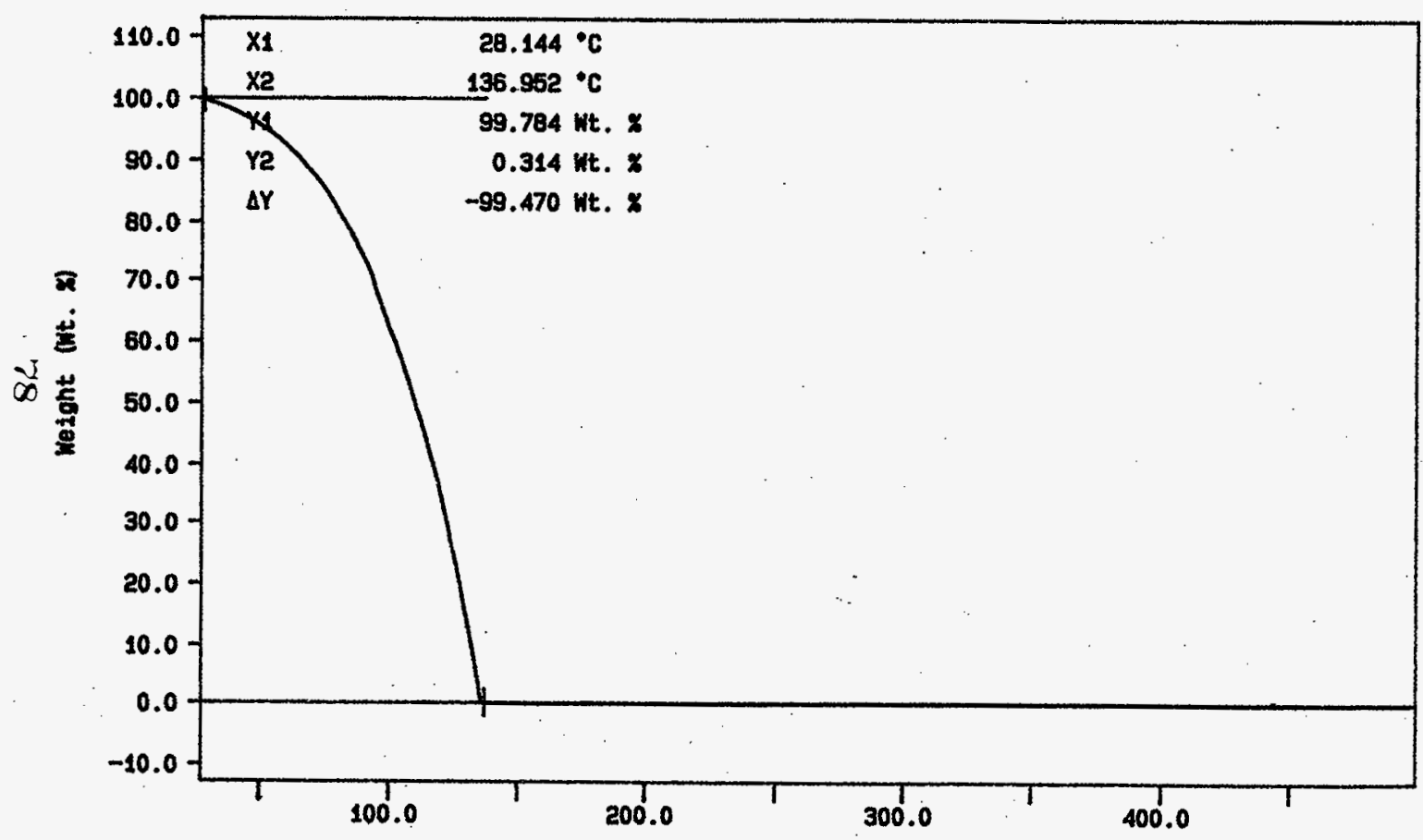

10C/MIN NR

Finept: sob:8 8 rawas: 0.0 min antes: 10.0 c/man

Teaperature $\left({ }^{\circ} \mathrm{C}\right)$ SM FutToN

PERIN-ELYER

7 Serteo Thermel Anelysib Systen sat vuI 19 01: 17: 33 1997 


\section{LABCORE Data Entry Template for Worklist\# 19382}

Analyst: $\quad$ OMF lustrument: TGAO 3 Book $130 N 8-A$

Method: LA-514-114 Rev/Mod $D-1$

Worklist Comment: BY-101 TGA, RUN UNDER N2. RCJ

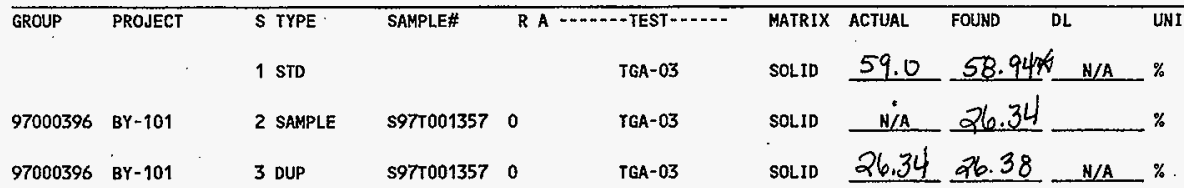

\section{Final page for worklist \# 19382}

Susi 72 . Sulton $7-18-97$

Validated 7/22/97\%rochelor

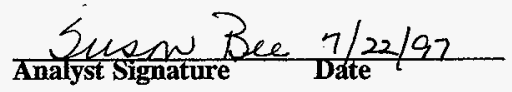

Units shown for QC (SPK \& STD) may not reflect the actual units. $D L=$ Detection Limit, $S=$ Worklist Slot Number, $R=$ Replicate Number, $A=$ Aliquot Code. 
Curve 1: TEA

F1Ie Info: TER071801 Fri U11 18 06: 47: 531997

Sample Wetght: 13.855 an

STD $10348-A$

SIGNATURE BELOW REPRESENTS CHEMICAL TECHNOLOGIST/CHEMIST THAT

COMALETEDVVERIFIED THE CALIBRATION/ANALYSIS ON PAGES 80 TO 82.

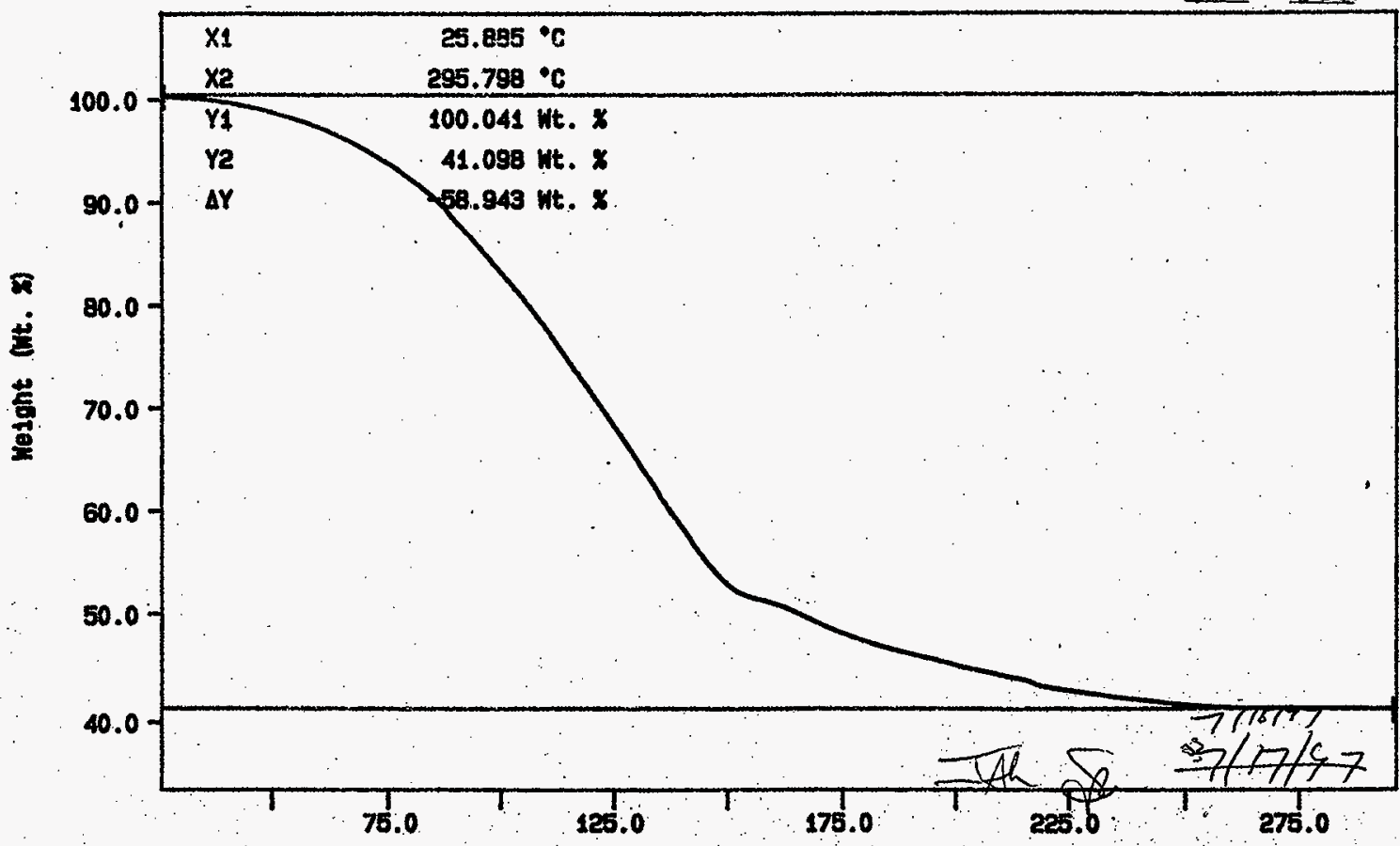

10C/MIN N2

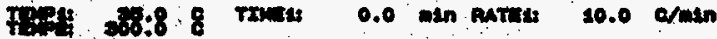

Temperature (*C)

D SpzLIN 


\section{Curve 1: TEA}

F11e info: SAM071812 Fri Jul 18 20: 06:56 1997

Sonple Vetoht: 23.494 mo

s97T001357

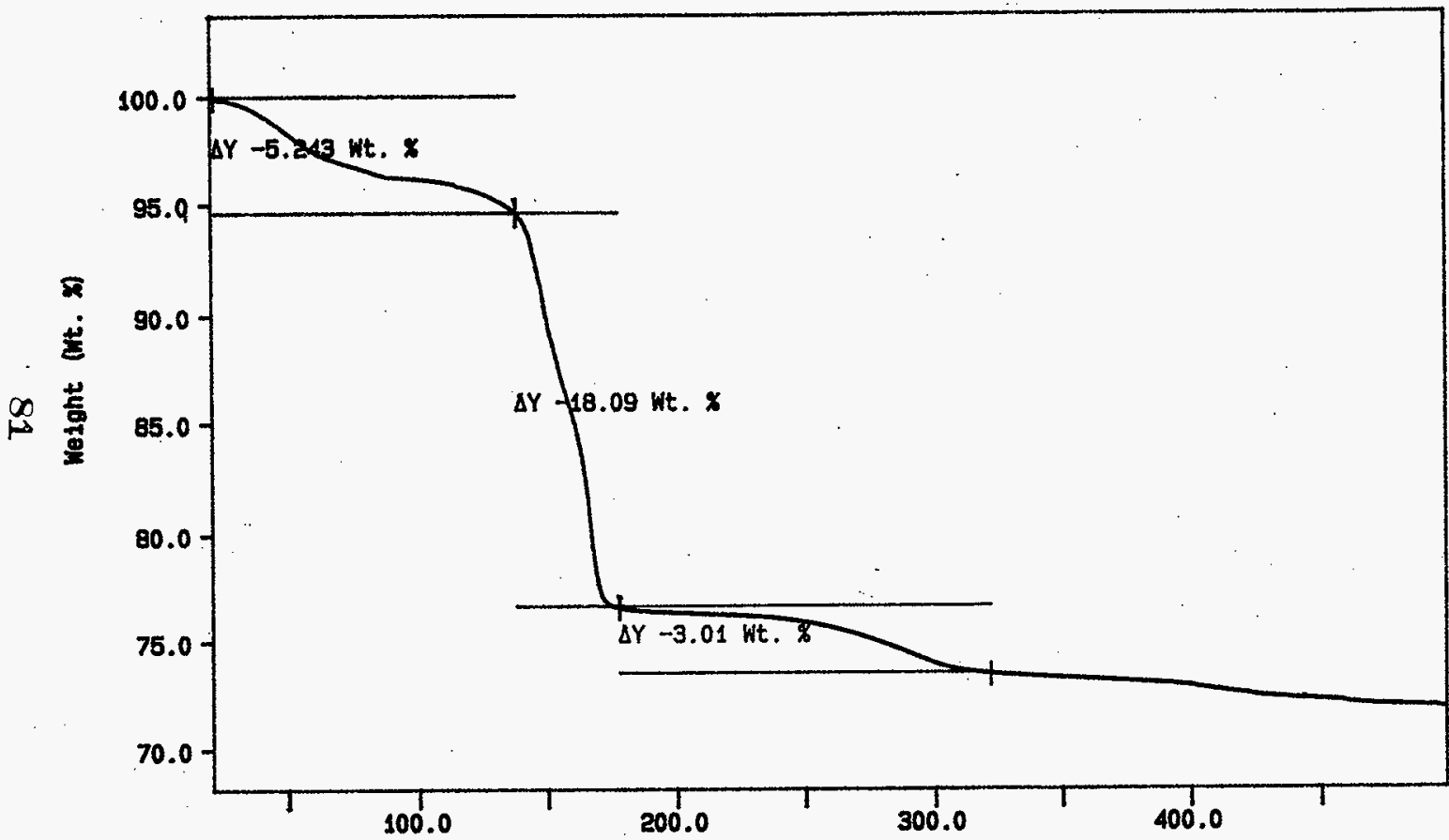

$10 \mathrm{C} / \mathrm{MIN}$ Ne

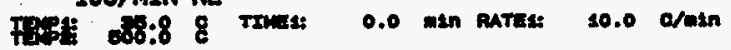

Temperature $\left({ }^{\circ} \mathrm{C}\right)$

SM FULTON

PERIKIN-EIMER

7 Serjes Thermal Analysis Systen Fp1 tu1 18 21: 11: 141997 
HNF-SD-WM-DP-258, REV. 0

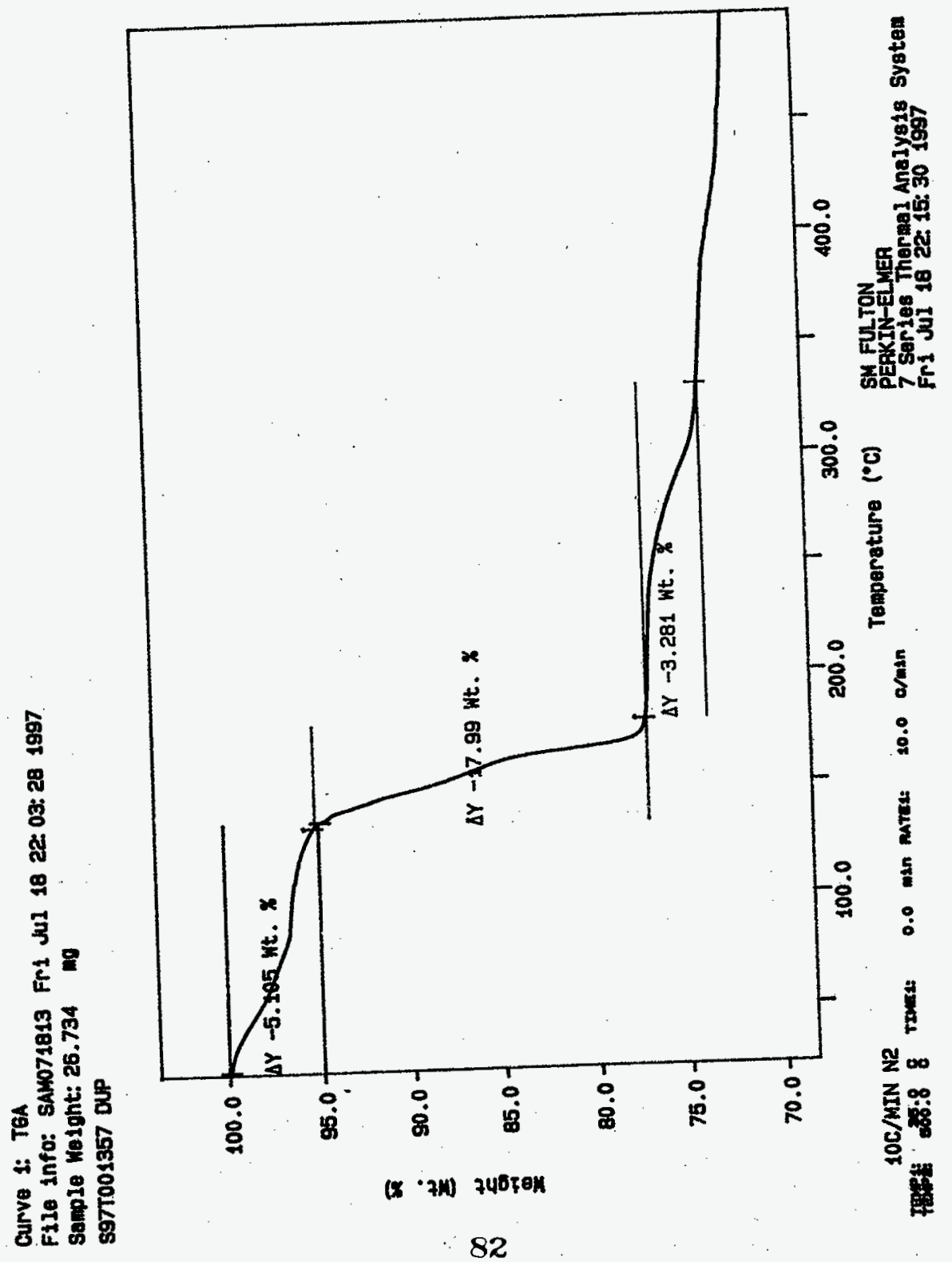




\section{LABCORE Data Entry Template for Worklist\# 19389}

Analyst: RDO _ Instrument: BA001_ Book \# 133/6A

Method: LA-510-112 Rev/Mod D-1

Worklist Comment: BY-101 SPG. RCJ

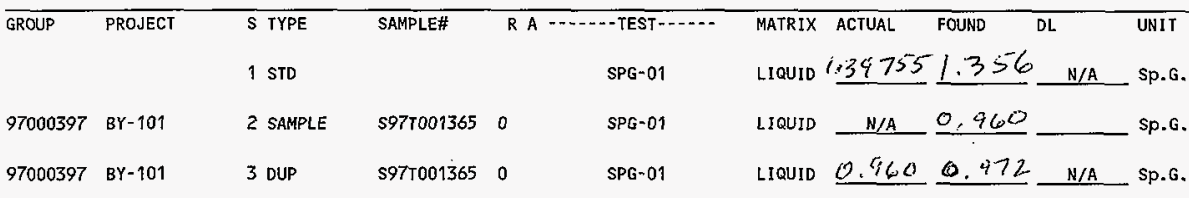

Final page for worklist \# 19389
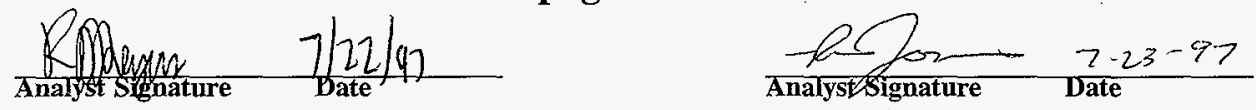

Oppraved ivechoesha

Units shown for $Q C$ (SPK \& STD) may not reflect the actual units. $D L=$ Detection Limit, $S=$ Worklist Slot Number, $R=$ Replicate Number, $A=$ Aliquot Code. 
HNF-SD-WM-DP-258, REV. 0

PLACE ANALYTICAL CARD IN BOX BELOW OR ATTACH TRAVELER,

\section{SPECIFIC GRAVITY : LA-510-112 (D-1)}

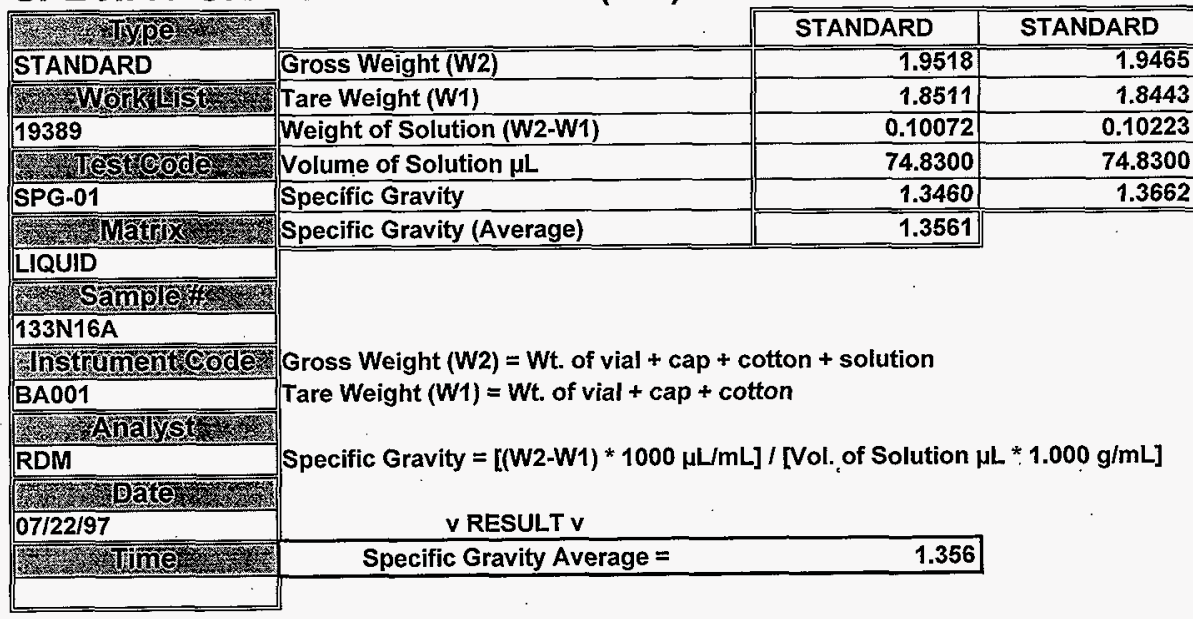

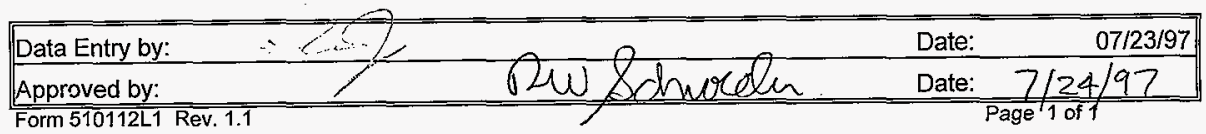

84 


\section{SPECIFIC GRAVITY : LA-510-112 (D-1)}

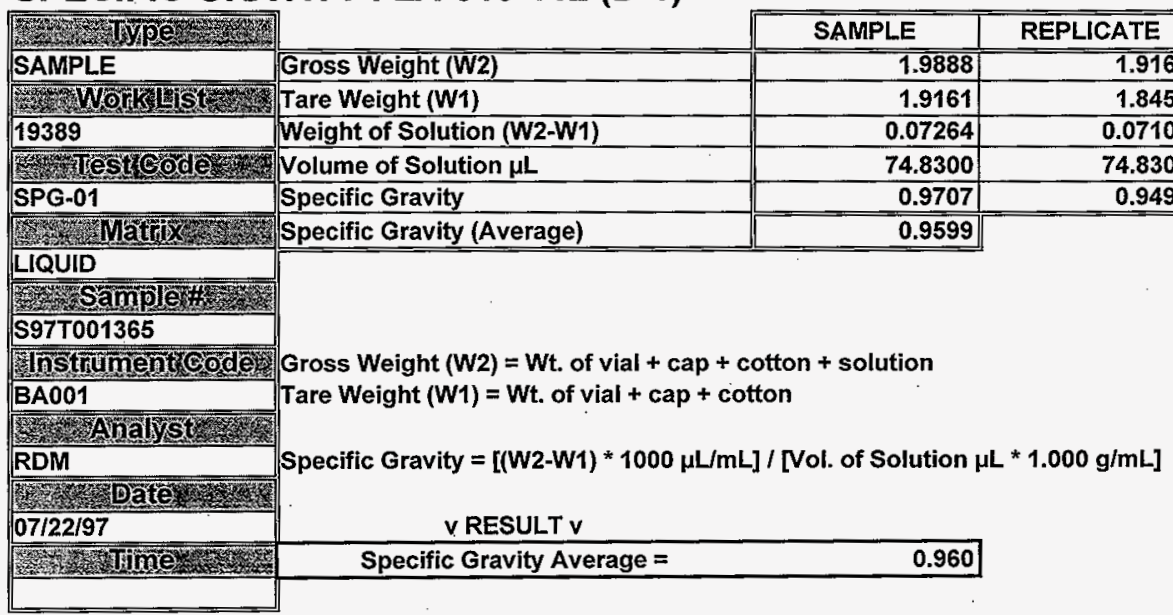

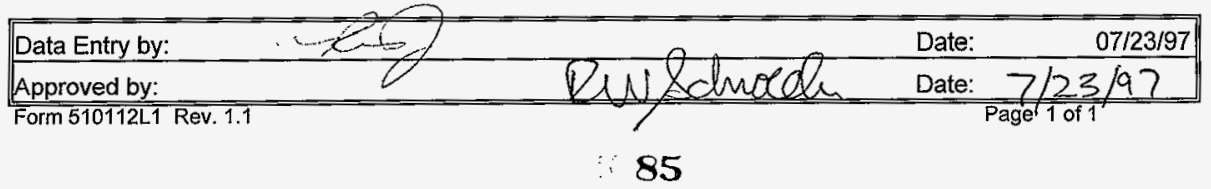




\section{SPECIFIC GRAVITY : LA-510-112 (D-1)}

\begin{tabular}{|c|c|c|c|}
\hline Fis & & DUPLICATE & REPLICATE \\
\hline DUPLICATE & Gross Weight (W2) & 1.9389 & 1.9936 \\
\hline Novirist & Tare Weight (W1) & 1.8666 & 1.9205 \\
\hline 19389 & Weight of Solution (W2-W1) & 0.0723 & 0.07311 \\
\hline 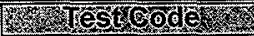 & Volume of Solution $\mu \mathrm{L}$ & 74.8300 & 74.8300 \\
\hline SPG-01 & Specific Gravity & 0.9662 & 0.9770 \\
\hline 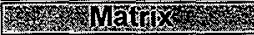 & Specific Gravity (Average) & 0.9716 & \\
\hline
\end{tabular}

\section{LIQUID}

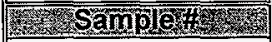

S97T001365

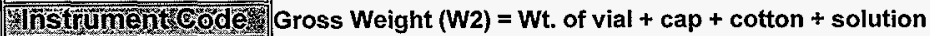

BA001

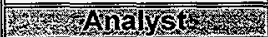

RDM

Fiver

$07 / 22 / 97$

Tare Weight $(W 1)=W t$. of vial + cap + cotton

Specific Gravity $=[(W 2-W 1) * 1000 \mu \mathrm{L} / \mathrm{mL}] /[$ Vol. of Solution $\mu \mathrm{L} * 1.000 \mathrm{~g} / \mathrm{mL}]$

\section{v RESULT v}

\begin{tabular}{|lll|}
\hline Data Entry by: & Date: \\
Approved by: & Date: $7 / 24 / 97$ \\
\hline Porm 510112L1 Rev.1.1 1 of 1
\end{tabular}




\section{LABCORE Completed Worklist Report for Worklist\# 18654}

Analyst: rag

Instrument: IC02

Book\# 220200A

Method: $14533-105$ Rev/Mod D-1

Worklist Comment: BY101, @IC-01 skm

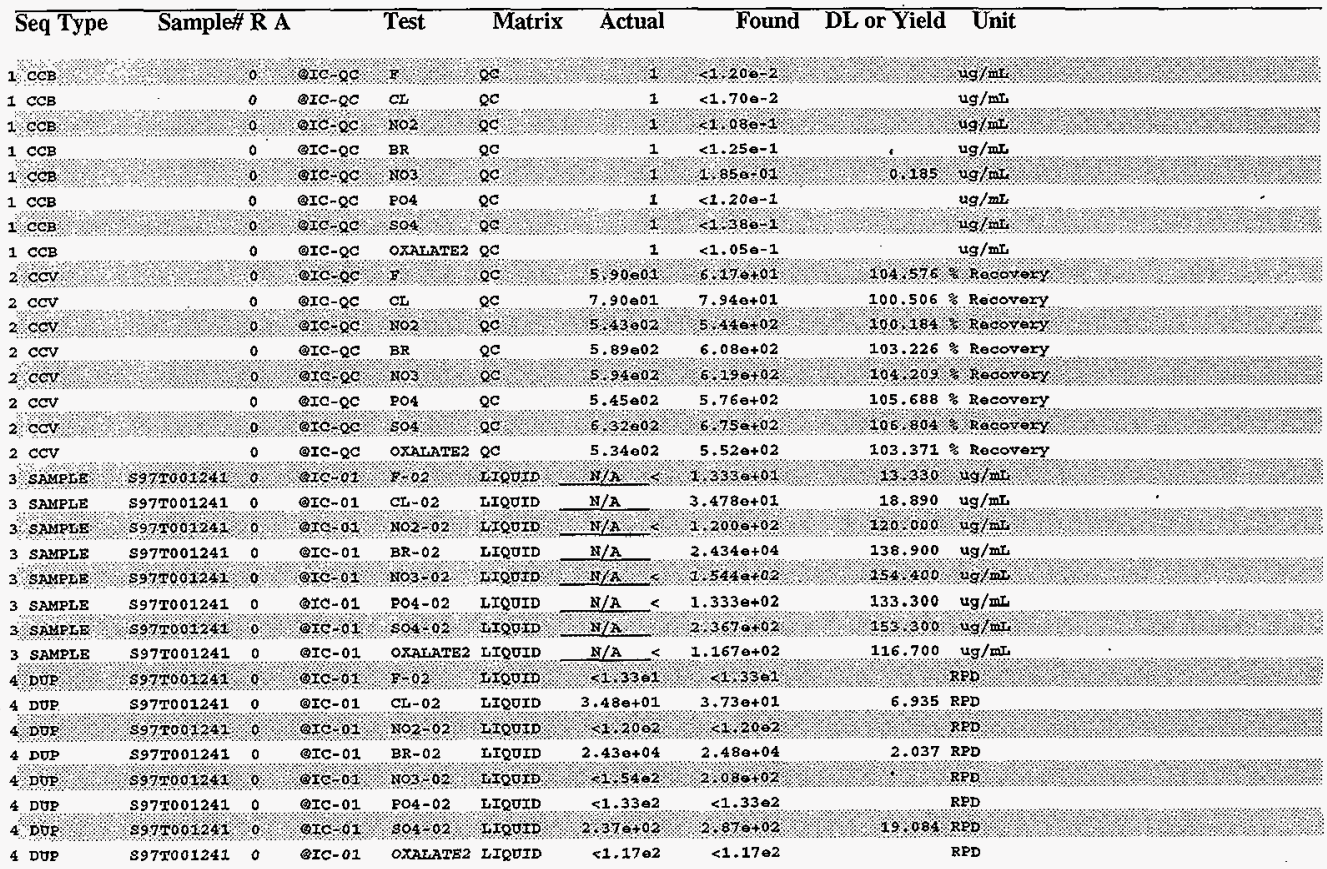

\section{Final page for worklist\# 18654}

Analyst Signature Date

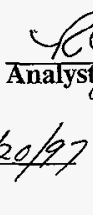

Units shown for $Q C(B L K / B K G)$ may not reflect the actual units. 


\section{LABCORE Data Entry Template for Worklist\# 18654}

Analyst: Ry Instrument: ICO 2 Book\# $12 \mathrm{~N} 2 \mathrm{O}^{-A}$

Method: LA-533-105 Rev/Mod D-1

Worklist Comment: BY101, @IC-01 skm

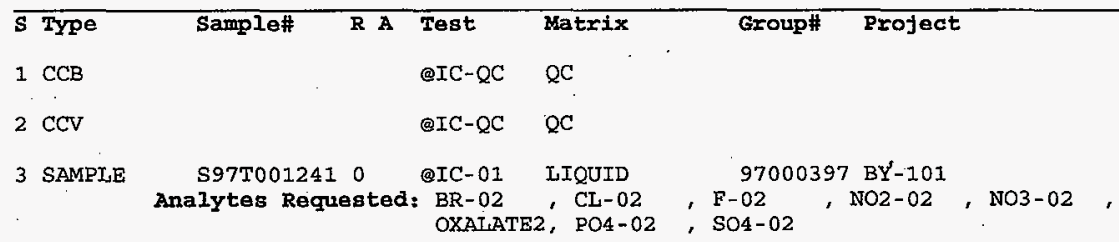

4 DUP $\quad$ S97T001241 $0 \quad$ QIC-01 LIQUID

\section{Final page for worklist \# 18654}

Raelen Yreen $06-18-97$.

Analyst Signature Date

Analyst Signature Date

Valedated $4 / 20 / 879 m 5$

Data Entry Comments:

$18654 \mathrm{JuN}, \mathrm{SCH}$

18654 Jun cs

$S=$ Worklist Slot Number, $\dot{R}=$ Replicate Number, $A=$ Aliquot Code. 
Sample Name: CCB

Data File :

Method

$C: \backslash D X \backslash D A T A \backslash 97061801 . D 06$

: C: \DX\METHOD \ANIONS :MET

ACI Address: 1 System: 1 Inject\# : 6

Analyst

S Column: AG4A/AS4A anion column

Date: $06 / 18 / 199714: 38: 43$

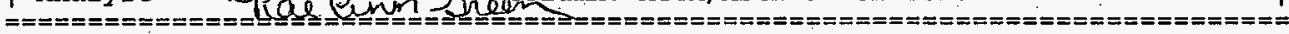

Calibration Volume Dilution Points Rate Start Stop Area Reject

External $\quad 1 \quad 1 \quad 3000 \quad 5 \mathrm{~Hz} \quad 0.00 \quad 10.00 \quad 50$

$\star * * * * * * * * * * * * * * * * * * * * * * * * * *$ Peak Report: All Peaks $* * * * * * * * * * * * * * * * * * * * * * * * * * * *$ Pk. Ret Component $\begin{array}{r}\text { Concentration } \\ \mathrm{ug} / \mathrm{ml}\end{array}$
Num Time Name Height Num Time Name ug/ml

$\begin{array}{rrrrrrr}1 & 0.89 & 0.000 & 11 & 52 & 1 & \\ 2 & 1.14 & 0.000 & 22 & 84 & 1 & \\ 3 & 1.39 & 0.000 & 21 & 112 & 1 & \\ 4 & 3.14 \text { nitrate } & 0.185 & 75 & 670 & 1 & -0.95 \\ 5 & 5.81 \text { sulfate } & 0.119 & 48 & 1140 & 1 & 1.81 \\ & & & 0.305 & 177 & 2058 & \end{array}$

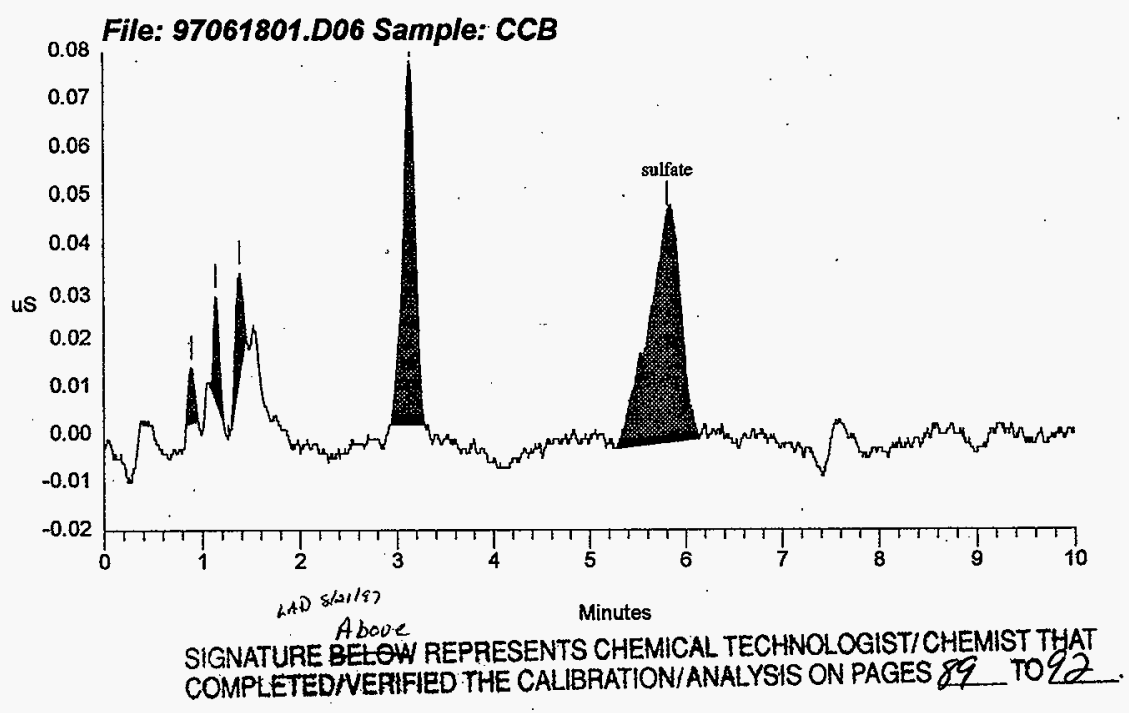


HNF-SD-WM-DP-258, REV. 0

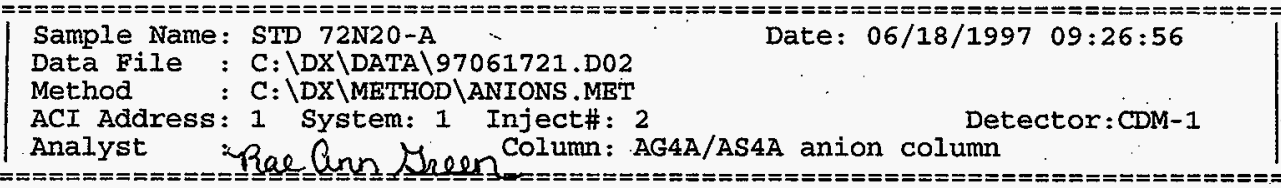

Calibration Volume Dilution Points. Rate Start Stop Area Reject

\begin{tabular}{|c|}
\hline External \\
\hline
\end{tabular}

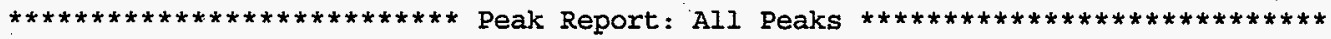

Pk. Ret Component Concentration. Height Area. Bl. \%Delta Num Time Name ug/ml

$\begin{array}{rrlrrrr}1 & 0.88 & 0.000 & 85 & 348 & 2 \\ 2 & 1.02 \text { fluoride } & 61.705 & 2044 & 11264 & 2 & 0.99 \\ 3 & 1.53 \text { chloride } & 79.419 & 2494 & 11270 & 1 & -0.87 \\ 4 & 1.82 \text { nitrite } & 543.637 & 10065 & 52776 & 1 & -2.50 \\ 5 & 2.69 \text { bromide } & 608.049 & 6269 & 38165 & 1 & -3.46 \\ 6 & 3.05 \text { nitrate } & 618.603 & 6942 & 50606 & 1 & -3.68 \\ 7 & 4.45 \text { phosphate } & 576.312 & 1832 & 22607 & 1 & 0.00 \\ 8 & 5.76 \text { sulfate } & 674.907 & 5563 & 74354 & 1 & 0.88 \\ 9 & 7.52 \text { oxalate } & 552.007 & 2541 & 46291 & 1 & -5.41\end{array}$

Totals

3714.640

37835

307682

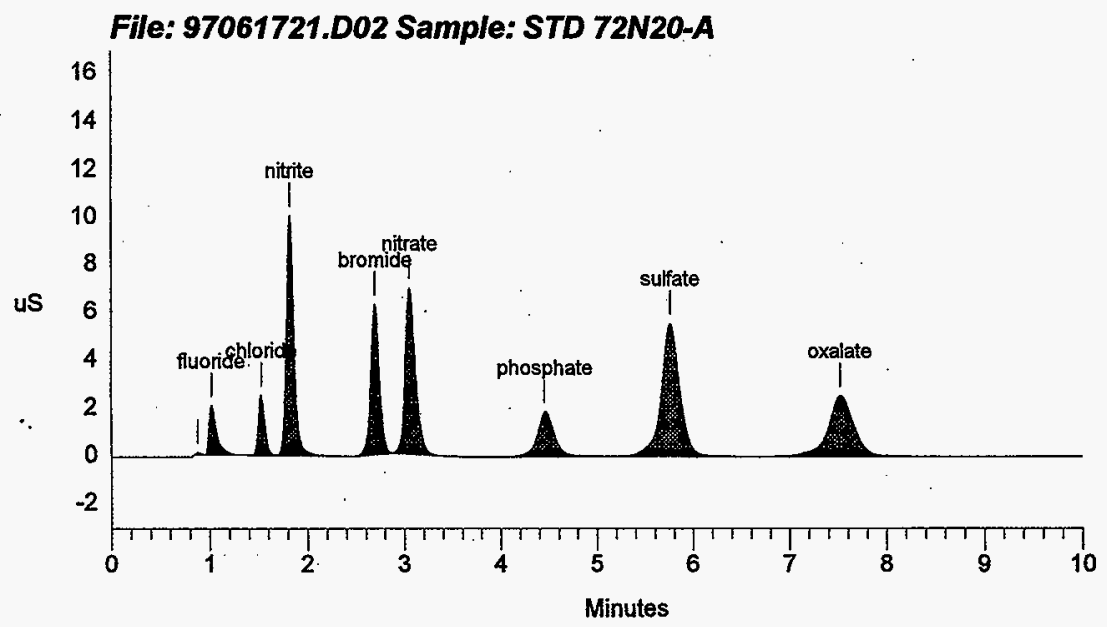




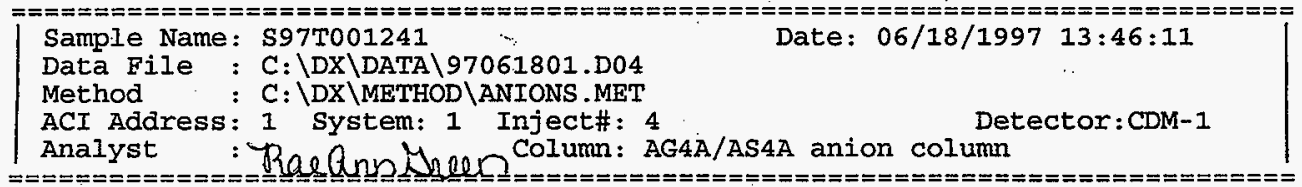

Calibration Volume Dilution Points Rate start stop Area Reject

External $11113000 \quad 5 \mathrm{~Hz} 0.00 \quad 10.00 \quad 50$

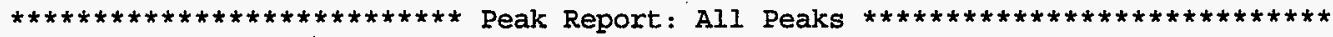

Pk. Ret Component $\quad \begin{gathered}\text { Concentration } \\ \text { ug/ml }\end{gathered}$. Height Area Bl. $\%$ Code
Nime Name

Num Time Name $\mathrm{ug} / \mathrm{m}$

$$
0.89
$$

0.000

55

0.000

95

1.38

0.000

18

1.53 chloride

34.785

2.71 bromide

24335.120

3.13 nitrate

145.778

5.81 sulfate

236.698

64

19665

39

100

301

557

84

292

150699

224

2164

Totals

24752.382

20035

154320

File: 97061801.D04 Sample: S97T001241

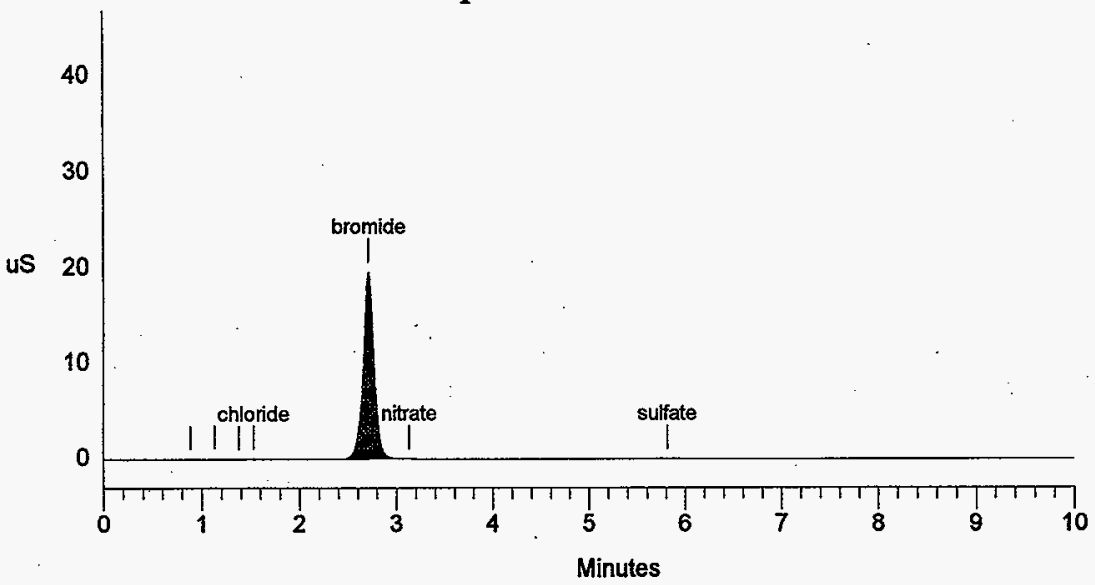


Sample Name: S97T001241 DUP-

Data File : C:\DX\DATA \97061801.D05

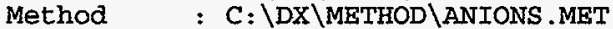

ACI Address: 1 System: 1 Inject\# : 5 Detector:CDM-1

Analyst : R

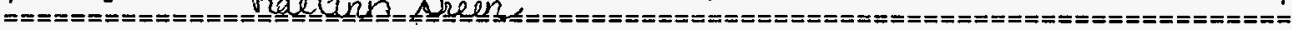

Calibration Volume Dilution Points Rate Start Stop Area Reject

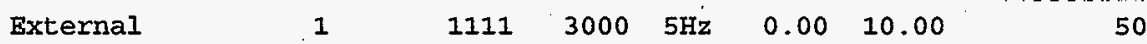

$* * * * * * * * * * * * * * * * * * * * * * * * * * *$ Peak Report: All Peaks $* * * * * * * * * * * * * * * * * * * * * * * * * * * *$

Pk. Ret Component $\quad \begin{gathered}\text { Concentration } \\ \mathrm{ug} / \mathrm{ml}\end{gathered}$
Num Time Name Height

(1)

$\begin{array}{lll}1 & 0.88 & \\ 2 & 1.13 & \\ 3 & 1.38 & \\ 4 & 1.54 & \text { chloride } \\ 5 & 2.71 & \text { bromide } \\ 6 & 3.13 & \text { nitrate } \\ 7 & 5.81 & \text { sulfate }\end{array}$

$\begin{array}{rrrr} & 0.000 & 67 & 397 \\ & 0.000 & 102 & 584 \\ 0.000 & 43 & 218 \\ 37.346 & 75 & 325 \\ & 24756.222 & 19884 & 153596 \\ & 207.622 & 96 & 683 \\ & 287.188 & 116 & 2662 \\ \text { Totals } & 25288.379 & 20383 & 158465\end{array}$

Totals

25288.379

20383

158465

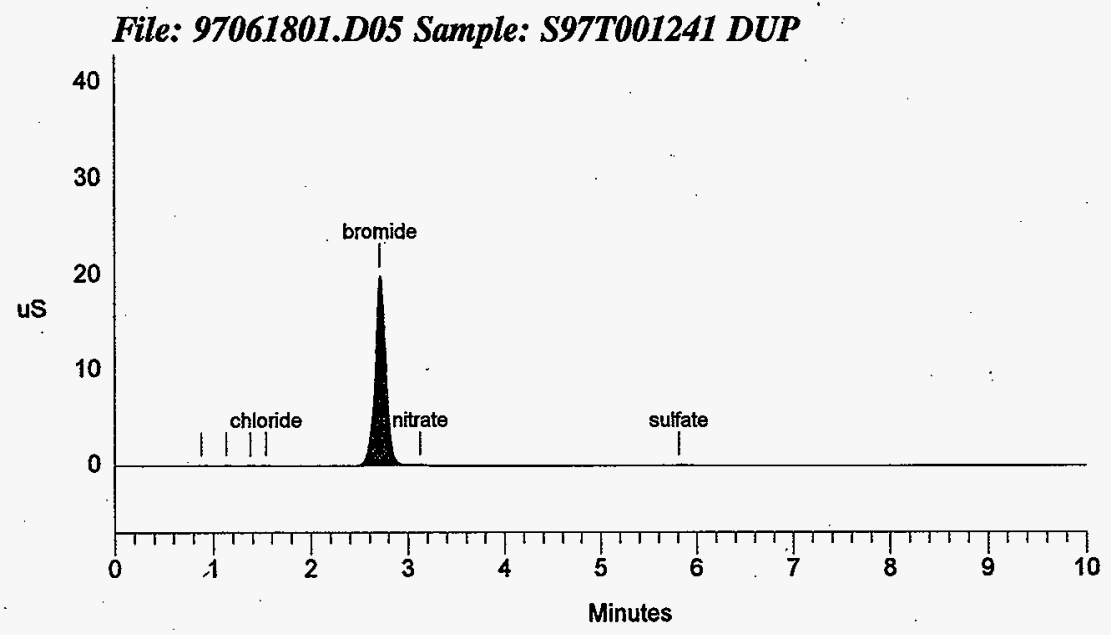




\section{LABCORE Completed Worklist Report for Worklist\# 19401}

\section{Analyst: $\mathrm{kgh}$}

Instrument: ICO1

Book\# 8301208

Method: $\quad A-533-105$ Rev/Mod $D-1$

Worklist Comment: BY-101 IC. RCJ

\begin{tabular}{|c|c|c|c|c|c|c|c|c|c|}
\hline Seq Type & Sample & $7 \mathbf{R A}$ & & Test & Matrix & Actual & Found DL or Yield & Unit & \\
\hline $1 \% \mathrm{cB}$ & & 0 & $9+00 \%$ & P & of & 1 & 14000 & 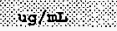 & \\
\hline $1 \mathrm{CCB}$ & & 0 & $\theta I c-Q c$ & CL & gc & 1 & $<1.70 e-2$ & $\mathrm{ug} / \mathrm{mr}$ & \\
\hline $1 / \mathrm{ceB}$ & & 0 & $8+C_{00 \mathrm{C}}$ & 302 & oc & 1 & 810001 & uglmim & \\
\hline $1 \mathrm{CCB}$ & & 0 & QIC-QC & $\mathbf{R R}$ & Qc & 1 & $<1.250-1$ & $\lg / \mathrm{m}$ & \\
\hline $1 \mathrm{ccs}$ & & 0 & GIC $Q 6$ & 403 & oct & 1 & 0,3901 & ug/ms & \\
\hline $1 \mathrm{ccB}$ & & 0 & arc-ec & PO4 & ge & 1 & $<1.200-1$ & $\mathrm{ug} / \mathrm{mr}$ & \\
\hline $1 \mathrm{ccB}$ & $\%$ & 0 & $\mathrm{OHO} 0 \mathrm{C}$ & $\mathrm{so4}$ & 06 & 1 & 43001 & lg/mit & \\
\hline $1 \mathrm{CCB}$ & & 0 & $Q I C-Q C$ & OXALATE2 & $Q c$ & 1 & $<1.05 e-1$ & $\mathrm{ug} / \mathrm{mL}$ & \\
\hline $2 \% c$ & & 0 & $\mathrm{OIC}-\mathrm{O}$ & 8 & 00 & 50001 & $6-63-01$ & $\beta / \operatorname{cop} \alpha x$ & \\
\hline $2 \mathrm{ccv}$ & & 0 & $Q I C-Q C$ & CL & Qc & 7.90001 & $7.35 e+01$ & \% Recovezy & \\
\hline $20 c v$ & & 0 & BIC-OC & $\mathrm{ro} 2$ & 00 & 5,44002 & $5110+02$ & 8 Rocovory & 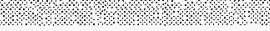 \\
\hline $2 \mathrm{ccv}$ & & 0 & $\triangle I C-Q C$ & Be & ec & 5.89002 & $5.600+02$ & : Resovary & \\
\hline $2 \mathrm{ccv}$ & & 0 & QIO 00 & no3. & 00 & 504002 & $5,5 \times 0,02$ & \% RosoreY & \\
\hline $2 \mathrm{ccv}$ & & 0 & aIC-QC & $\mathrm{PO4}$ & $\mathrm{gc}$ & 5.45002 & $5.390+02$ & $\%$ Recovery & \\
\hline $2 \mathrm{cov}$ & & 0 & Qre- & 804 & $\infty \mathrm{c}$ & 6.3260 & $5880+02$ & $6 \mathrm{Rec} 0-01 \mathrm{\gamma}$ & \\
\hline $2 \mathrm{ccv}$ & & 0 & $\operatorname{arc}-\mathrm{Qc}$ & OXARATE2 & $Q c$ & 5.33002 & $5.060+02$ & \% Recovery & \\
\hline $38 \mathrm{LMK}$ RRBP & & 2 & $0 \times 0-01$ & E 02 & sorso & 13 & $0100-2$ & ug/g & \\
\hline 3 BENK-PRER & & 0 & OIC-01 & CL- 02 & SOLID & 1 & $<1.700-2$ & $u g / g$ & \\
\hline $3, P L N A P R E P$ & & 0 & $0 r c-a 1$ & $\mathrm{NO}_{2} \mathrm{OO}_{2}$ & sotxpo & 1 & $2260-01$ & $4 \mathrm{qg} / \mathrm{g}$ & \\
\hline 3 BLNK-PREP & & 0 & QIC-01 & $\mathrm{BR}-02$ & SOLID & 1 & $<1.250-1$ & $\mathrm{ug} / \mathrm{g}$ & \\
\hline 3 BLNK RREP & & 0 & OIC 01 & $\mathrm{No}-02$ & sontog & $1 \%$ & $11390-1$ & $40 / 9$ & \\
\hline 3 BLAK-PREP & & 0 & arc-01 & $\mathrm{POA}-02$ & SOLID & 1 & $<1.200-1$ & $\mathrm{ug} / \mathrm{g}$ & \\
\hline $3 \mathrm{BENK}-\mathrm{PREP}$ & & 0 & $6 \mathrm{CC}-01$ & $500-02$ & SOLID & 1 & $1,380-1$ & $19 \%$ & \\
\hline 3 BLNK-PREP & & 0 & QIC-01 & OXALATE2 & SOLID & 1 & $<1.05 e-1$ & $\mathrm{ug} / \mathrm{g}$ & \\
\hline 4 SAMPLE & 8979001360 & $0 \mathrm{n}$ & $0 I 0-01$ & 802 & solsp & 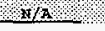 & $1+1340+03$ & $140 / 9$ & \\
\hline 4 SAMPLE & 8977001360 & $0 \%$ & arc-01 & $c x-02$ & SOzTD & $N / \mathrm{A}<$ & $1.713 \theta+02$ & $u g / g$ & \\
\hline 4 S 4 MRE & 8979009360 & $0 \mathrm{H}$ & $8>C-01$ & $\mathrm{NO} 2-02$ & SOLSD & $3 / A \% 5$ & 10880003 & 4g/9 & \\
\hline 4 SAMPLE & 5977001360 & $0 w$ & खIC-01 & $\mathrm{BR}-02$ & SOLID & $N / A$ & $2.314 \theta+03$ & $\mathrm{ug} / \mathrm{g}$ & \\
\hline $4 \mathrm{SAMPBE}$ & 5979001360 & $0 \mathrm{~W}$ & OIQ 01 & $103-02$ & $\mathrm{socho}$ & $0 \%$ & $4,730+05$ & $109 / 9$ & \\
\hline 4 SAMPLE & $\$ 9710001360$ & ด & बIC-01 & PO4-02 & SOLID & $N / \mathbf{A}$ & $4.481 e+04$ & $\mathrm{ug} / \mathrm{g}$ & \\
\hline 2 SAMPLE & $\$ 970001360$ & $0, \mathrm{H}$ & $\mathrm{OxO}_{01}$ & $\mathrm{~s} 0 \mathrm{~A}-02$ & SOLAD & $\mathrm{H} / \mathrm{K}$ & 1.3910003 & ug/o & \\
\hline 4 SAMPLE & $\$ 97 T 001360$ & $0 \mathrm{~N}$ & QIC-01 & OXATAATEZ & SOLID & $n / A$ & $7.849 \theta+03$ & $\mathrm{ug} / \mathrm{g}$ & \\
\hline 500 & $\$ 90 \% 001360$ & $00 \mathrm{~K}$ & $0 \times 001$ & $\mathrm{Fo2}$ & solip & $4,100+03$ & 30086 & RPD & \\
\hline $5 \mathrm{DVP}$ & $597 T 001360$ & $0 \mathrm{~W}$ & QIC-01 & $\mathrm{CL}-02$ & SOLID & $<1.7102$ & $<1.73 \Leftrightarrow 2$ & RPD & \\
\hline $5 \% D O P$ & $897 \mathrm{roO}_{3} \mathrm{Ho}$ & $0 \%$ & $0 \times 01$ & 00202 & soLfo & $\alpha 1,0903$ & 4,2003 & $\mathrm{RpD}$ & \\
\hline 5 DUR & $897 T 001360$ & ow & BIC-01 & $\mathrm{BR}-02$ & SOLID & $2.31 e+03$ & $2.26 e+03$ & RPD & \\
\hline 5 DOP & 5079002060 & $0 \%$ & 01001 & 403202 & solyp & $4+1+8+05$ & $3270+05$ & $\mathrm{RpD}$ & \\
\hline 5 DUP & $897 \mathrm{~T} 001360$ & OW & QIC-01 & PO4-02 & SOLID & $4.48 e+04$ & $6.45 e+04$ & RPD & \\
\hline $5 \mathrm{DOP}$ & 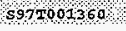 & $0 \%$ & $0 \times 001$ & $504-02$ & SOLAD & K103963 & 01,4103 & $\mathrm{RPD}$ & \\
\hline $5 \mathrm{DUP}$ & 5975001360 & ow & QIC-01 & OXALATE2 & SOLID & $7.85 e+03$ & $7.940+03$ & RPD & \\
\hline $6 \mathrm{SPK}$ & 8971001360 & $0 \mathrm{w}$ & $010-01$ & $=02$ & soLrD & 580000 & $5200+01$ & & \\
\hline
\end{tabular}

Units shown for $Q \overline{Q C}(B L K / B K G)$ may not reflect the actual units. 


\section{LABCORE Completed Worklist Report for Worklist\# 19401}

\begin{tabular}{|c|c|c|c|c|c|c|c|c|c|}
\hline Seq Type & Sample & $\bar{R}$ & & Test & Matrix & Actual & Found DL or Yield & 1 Unit & \\
\hline $5 \mathrm{SPK}$ & S97T001360 & 0 & $W$ WC-0I & CL-02 & SOLID & 7.90001 & $7.150+01$ & \% Recovery & \\
\hline 6 SEK & 5975001360 & $0 \mathrm{~h}$ & $w \quad \operatorname{IC-OI}$ & tro2-02 & SOLID & 5.44902 & $5.05 e+02$ & \% Recovery & \\
\hline 6 spre & 8970001360 & 01 & Xorcoro & $\mathrm{BR}-0 \mathrm{Z}$ & SOLPD & 5,89002 & $5350+02$ & $\% 1000017$ & ma \\
\hline 6 SPK & S97T001360 & 0 & $A$ aIC-0I & $\mathrm{NO3-02}$ & SOLID & 5.94002 & $2.280+02$ & \% Recovery & \\
\hline $6 \mathrm{spK}$ & 8997001860 & 0 & M $4 \mathrm{cl} 01$ & $\mathrm{pos} 02$ & solito & 54502 & $505-102$ & R Roporexy & \\
\hline $6 s_{P K}$ & $\mathrm{~S} 97 \mathrm{~T} 001360$ & on & $w$ IC-01 & so4-02 & SOLID & 6.32002 & $5.810+02$ & * Recovery & \\
\hline $65 \mathrm{sK}$ & 5922001860 & 0 & $N \& T_{C} 0 \mathrm{I}$ & OXALAT2 & SOLTD & $5 \times 30.02$ & $40960+02$ & $1, \mathrm{Recovexh}$ & \\
\hline 7 SAMPLE & 5972001354 & 0 & $W \quad 0 I C-01$ & $F-02$ & SOLID & $\mathrm{H} / \mathrm{A}$ & $8.1990+03$ & $4 g / g$ & \\
\hline $7 \mathrm{SAMES}$ & s97T001364 & 0 & $W / \mathrm{rc}_{01}$ & $\mathrm{ct} 02$ & Solin & $\mathrm{N} / \mathrm{A} / \mathrm{s}$ & $1,7520+02$ & $2 \mathrm{lg} / \mathrm{g}$ & \\
\hline 7 SAMPLE & S97T001364 & 0 & P $\because 2 C-01$ & $\mathrm{NO2}-02$ & SOLID & $\mathbb{N} / \mathrm{A}<$ & $1.113 \theta+03$ & $4 g / g$ & \\
\hline 7 sAMPIS & s9710001364 & 0 & मब्Sc- 01 & $\mathrm{BR}-02$ & SOLID & $\mathrm{N} / \mathrm{A}, \mathrm{L}$ & $12880+03$ & ug $/ 9$ & \\
\hline 7 SAMPLE & $\$ 97 \pi 001364$ & 0. & $w$ IC-01 & No3-02 & SOLID & $N / A$ & $3.962 e+05$ & $\mathrm{ug} / \mathrm{g}$ & \\
\hline 7 AMPLE & $897 \times 001364$ & 0 & W $110-01$ & poo -02 & SOKTD & $x / x$ & $8,8180,04$ & ugls & \\
\hline 7 SAMPLE & S97T001364 & 0 & $w$ बrc-01 & so4-02 & SOLID & N/A $<$ & $1.422 \theta+03$ & $\mathrm{ug} / \mathrm{g}$ & \\
\hline 7 spingt: & 5979001364 & 01 & $\$ 8 \times c 01$ & $0 \times$ NATE2 $_{2}$ & Solno & N/A & $6452 \theta 03$ & $\mathrm{ug} / 9$ & \\
\hline $8 \mathrm{DUP}$ & $597 T 001364$ & 0 & $w$ eIc-01 & $E-02$ & SOEID & $8.200+03$ & $8.98 \theta+03$ & RPD & \\
\hline $800 R$ & 5972001364 & 0 & $4010-01$ & 0402 & SOL $T$ D & 81,7502 & $\times 12102$ & $\mathrm{BPD}$ & \\
\hline \& DUP & S97T001364 & 0 & $W @ I C-01$ & so2-02 & SOLID & $<1.11 \mathrm{e} 3$ & $<1.0903$ & RED & \\
\hline 8 opp: & 5979001364 & 0 & $W 0 r e-01$ & $\mathrm{BR}-02$ & SOSTD & $\times 1<2903$ & $<1,2603$ & RpD & \\
\hline $8 D O P$ & 8979001364 & 0 & W STC-01 & $\mathrm{NO3-02}$ & Sor.to & $3.960+05$ & $3.77 \theta+05$ & RPD & \\
\hline $8 \mathrm{DOP}$ & 8970001364 & 00 & W $8 \mathrm{xO}_{0}$ & 80402 & solin & 8.820104 & $1440+04$ & ppD & \\
\hline 8 DUP & S97T001364 & 0 & $w \quad 0 I C-01$ & $504-02$ & SOLID & $<1.42 \in 3$ & $<1.39 \theta_{\theta}$ & $R F D$ & \\
\hline 800 & $\$ 97 \mathrm{I} 00 \mathrm{Y} 364$ & 0 & $6 \times 8-\infty$ & $0 \times A \times 4+2$ & SOLTD & $6,450+03$ & $6590+00$ & RPD & \\
\hline SPK & 5979001364 & 0 & $w$ arc-01 & $8-02$ & SOLID & 5.90001 & $6.379+01$ & $\%$ Recovery & \\
\hline $9 \mathrm{spK}$ & 8990001364 & 01 & $\mathrm{WOrcol}$ & $\mathrm{oL}-02$ & solso & 700001 & $7800+01$ & \%Roooxory & \\
\hline g SPK & $597 T 001364$ & 0 & $w$ IC-01 & NO2-02 & SOLID & $5.44 \times 02$ & $5.35 e+02$ & \% Recovery & \\
\hline $9 \mathrm{SPR}$ & 9972001364 & 0 & 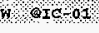 & $\mathrm{BR}-\mathrm{dz}$ & SOLID & 508902 & $5130+02$ & . $\mathrm{B}(\mathrm{coval}$ & \\
\hline $9 \mathrm{SPK}$ & 5977001364 & 0 & $w \quad$ aIc-01 & $\mathrm{NO3-02}$ & SOLID & 5.94002 & $7.52 e+02$ & \% Recovery & \\
\hline 9 spe & $\$ 5970001364$ & 0 & Wtøc-01 & $004-02$ & sotro & 565602 & $62260+00$ & \% Becotory & \\
\hline 9 SPK & $\$ 97 T 001364$ & 0 & $w \quad \operatorname{arc}-01$ & $504-02$ & SOLID & $6.32 \times 02$ & $6.100+02$ & $\%$ Recovery & \\
\hline 359 & 5975001364 & 0 & $\$ 010-1$ & $0 \times A=\mathrm{TR}_{2}$ & SOLID & 333002 & 528002 & $\% R \bullet d \circ$ ory & \\
\hline
\end{tabular}

\section{Final page for worklist\# 19401}

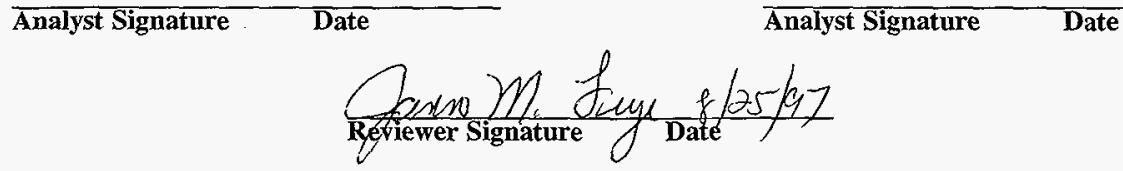

Units shown for $Q \overline{Q C}(B L K / \overline{B K G})$ may not reflect the actual units. 


\section{LABCORE Data Entry Template for Worklist\# 19401}

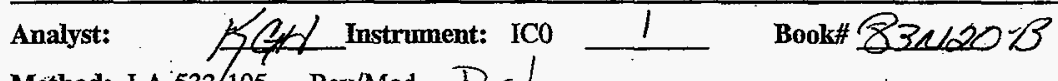

Method: LA-533/105 Rev/Mod D $D-1$

Worklist Comment: BY-101 IC. RCJ

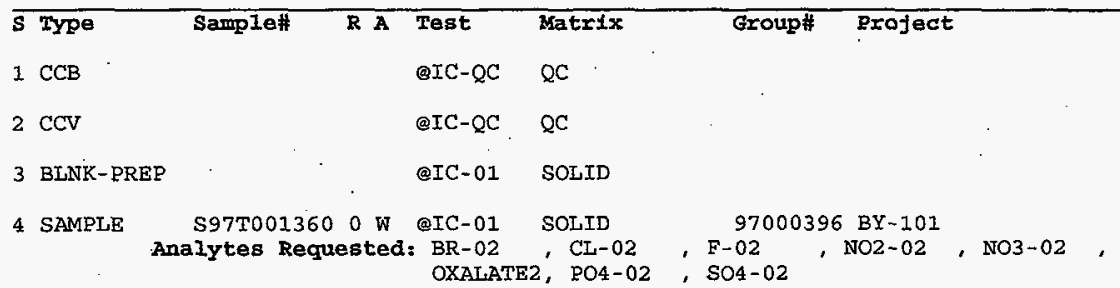

5 DUP S97T001360 $0 \mathrm{~W}$ QIC-01 SOLID

E SPR . S97T001360 OW.QIC-01 SOLID

7 SAMPLE S97T001364 $0 \mathrm{~W}$ @IC-01 SOLID $97000397 \mathrm{BY}-101$

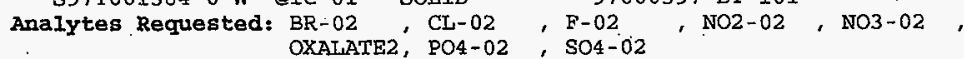

8 DUP S97T001364 OW QIC-01 SOIID

9 SPK S97T001364 O W QIC-01 SOLTD

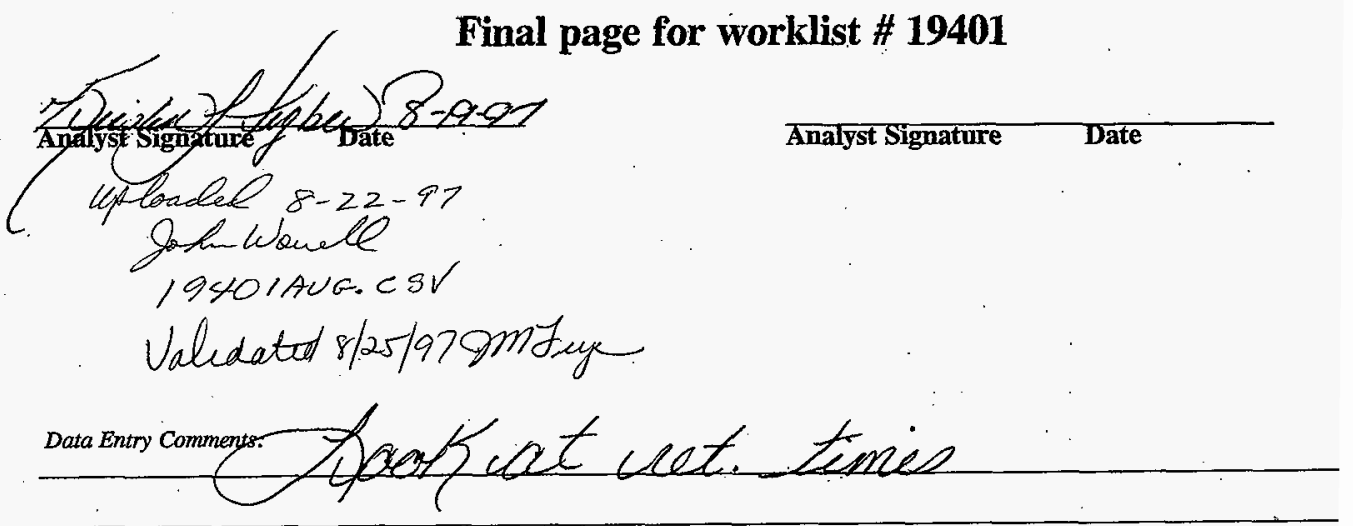

$S=$ Worklist Slot Number, $R=$ Replicate Number, $A=$ Aliquot Code. 


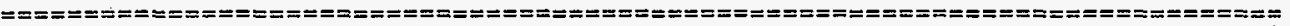

Sample Name: BLANK

Data File : C: DDX $\backslash$ DATA $\backslash 97081921 . D 01$

Method : C: \DX\METHOD $\backslash$ KIT.MET

ACI Address: 1 System: 1 Inject\#: 1

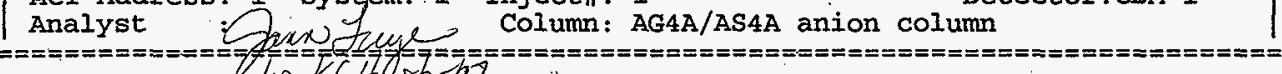

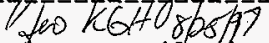

Calibration Volume Dilution Points Rate start stop Area Reject

External $\quad 1 \quad 30005 \mathrm{~Hz} 0.00 \quad 10.00 \quad 30$

$* * * * * * * * * * * * * * * * * * * * * * * * * * *$ Peak Report: All Peaks $* * * * * * * * * * * * * * * * * * * * * * * * * * * *$

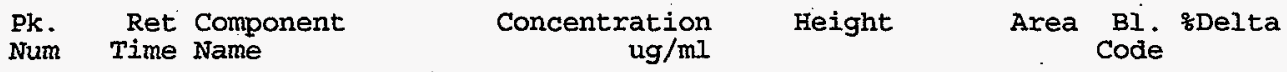

$\begin{array}{lllllll}1 & 1.33 & 0.000 & 37 & 182 & 1 & -2.88 \\ 3 & 1.57 \text { chloride } & -0.016 & 50 & 186 & 1 & -2.94 \\ 4 & 3.37 \text { nitrate } & -0.019 & 53 & 358 & 1 & -1.94 \\ 4.24 \text { sulfate } & -0.255 & 27 & 427 & 1 & -2.50\end{array}$

Totals $\quad-0.290 \quad 167 \quad 1152$

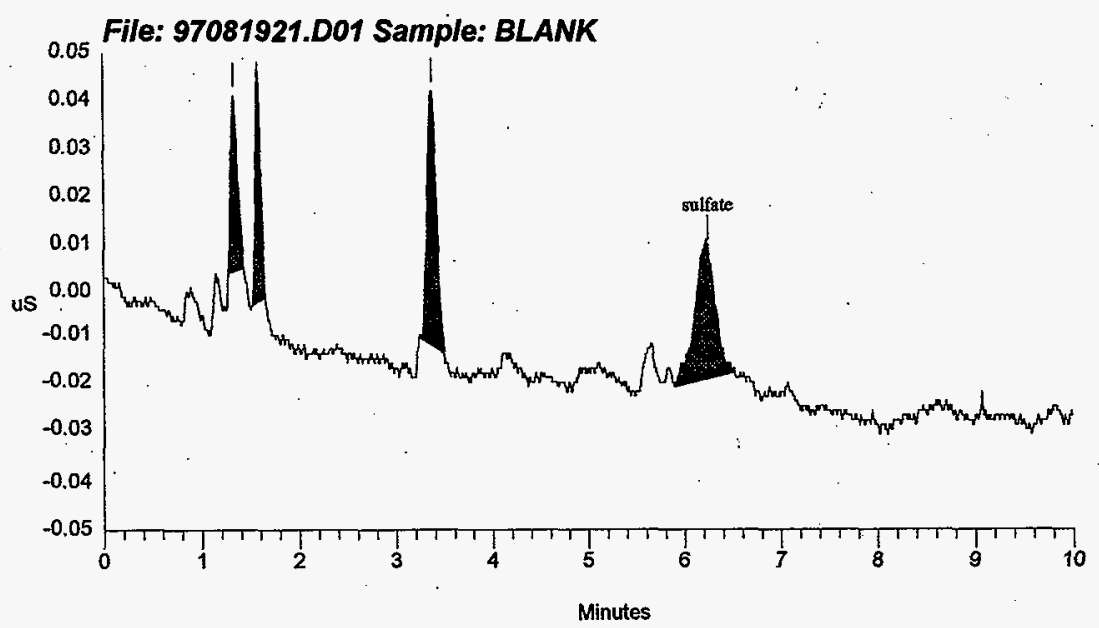

SIGNATURE ABOVE REPRESENTS CHEMICAL TECHNOLOGIST/CHEMIST THAT ONPLETED/NERIFIED THE CALIBRATION/ANALYSIS ON PAGES TO 


\section{$H N E=8 D-W M-D P-258, R E V .0$}

Data Reprocessed On 08/22/1997 10:13:47

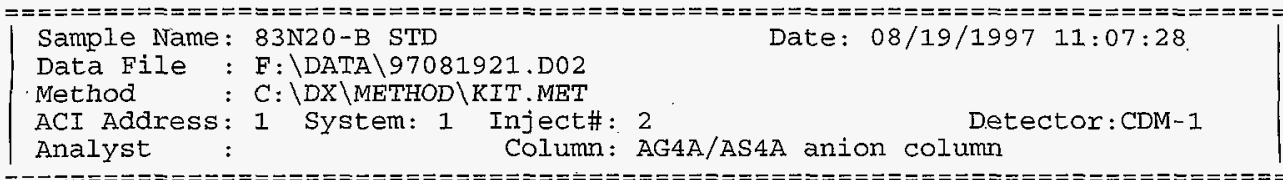

Calibration Volume Dilution Points Rate start Stop Area Reject

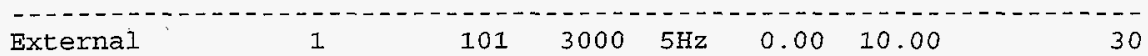

$* * * * * * * * * * * * * * * * * * * * * * * * * * *$ Peak Report: All Peaks $* * * * * * * * * * * * * * * * * * * * * * * * * * * *$

Pk. Ret Component Concentration Height Area Bl. $\div D e l t a$

Num Time Name ug/ml Code

\begin{tabular}{|c|c|c|c|c|c|c|c|}
\hline 1 & 0.85 & & 0.000 & 94 & 395 & 1 & \\
\hline 2 & 1.01 & fluoride & 66.301 & 2743 & 13390 & 3 & -4.13 \\
\hline 3 & 1.31 & & $0: 000$ & 25 & 127 & 4 & \\
\hline 4 & 1.55 & chloride & 73.488 & 2302 & 10202 & 1 & -4.12 \\
\hline 5 & 1.88 & nitrite & 513.167 & 9270 & 46826 & 1 & -4.08 \\
\hline 6 & 2.87 & bromide & 560.168 & 5320 & 33340 & 1 & -4.76 \\
\hline 7 & 3.27 & nitrate & 554.374 & 5681 & 44188 & 1 & -0.10 \\
\hline 8 & 4.67 & phosphate & 538.953 & 1806 & 20057 & 1 & -5.41 \\
\hline 9 & 6.08 & sulfate & 588.153 & 3941 & 61656 & 1 & -5.00 \\
\hline 10 & 8.05 & oxalate & 505.732 & 2202 & 39691 & 1 & -5.03 \\
\hline
\end{tabular}

Totals $\quad 3400.337 \quad 33385 \quad 269872$

File: 97081921.D02 Sample: 83N20-B STD

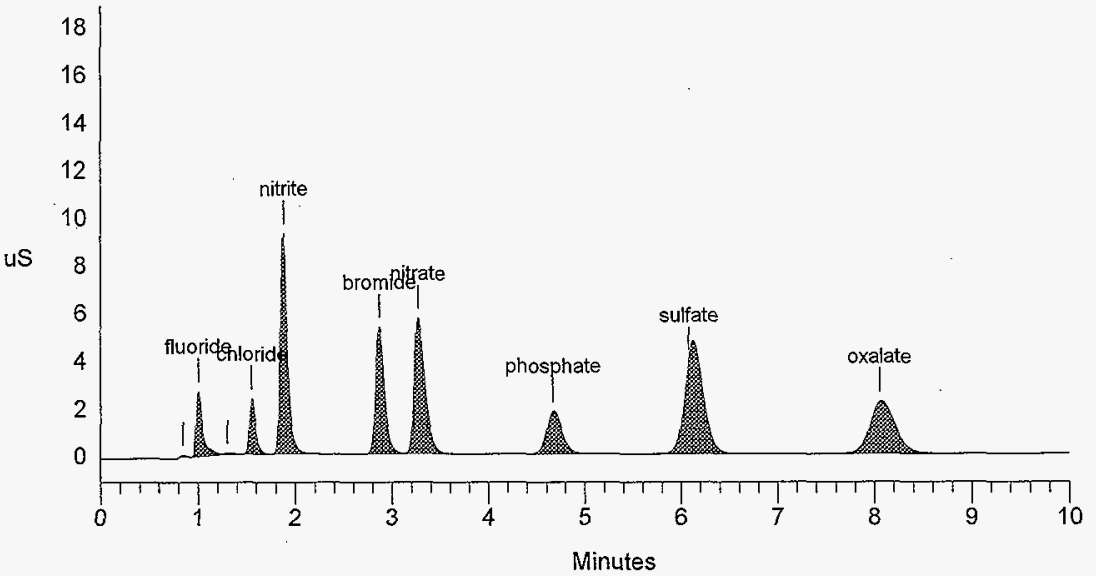


Sample Name: PREP-BLANK

Data File : C: \DX\DATA \.97081921.D03

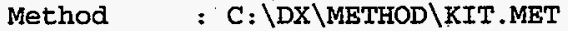

ACI Address: 1 System: 1 Inject\# : 3

Analyst

Column: AG4A/AS4A anion column

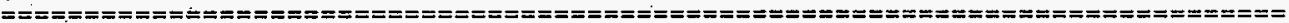
Calibration Volume Dilution Points Rate start stop Area Reject

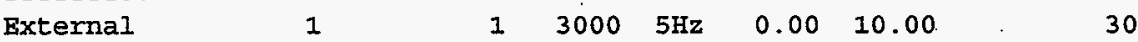

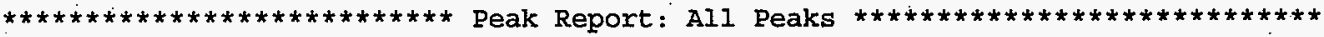

Pk. Ret Component Concentration Height Area Bl. $\%$ Delta

Num Time Name $\mathrm{ug} / \mathrm{ml}$ Code

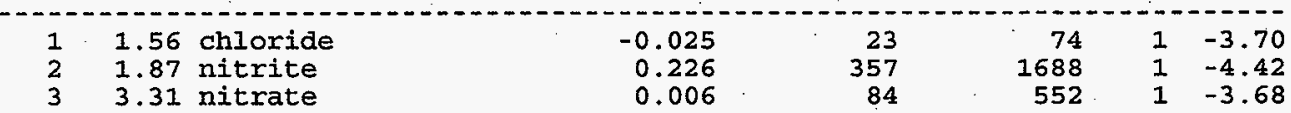

Totals $\quad 0.207 \quad 464 \quad 2314$

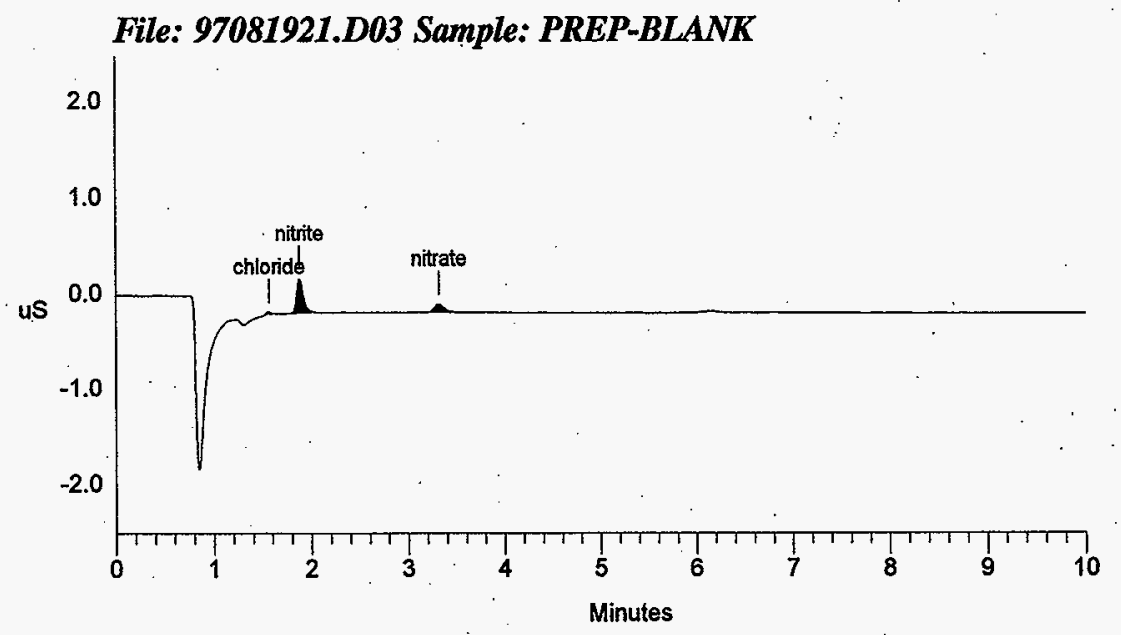


Data Reprocessed On 08/22/1997 10:15:19

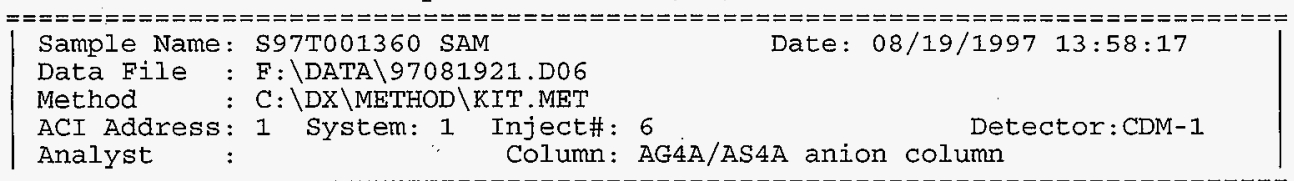

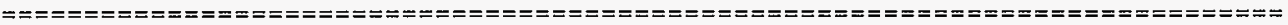

Calibration Volume Dilution Points Rate start stop Area Reject

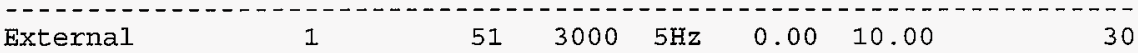

$* * * * * * * * * * * * * * * * * * * * * * * * * * *$ Peak Report: All Peaks $* * * * * * * * * * * * * * * * * * * * * * * * * * * *$ $\begin{array}{lrrr}\text { Pk. Ret Component } & \text { Concentration } & \text { Height } & \text { Area Bl. } \\ \text { Num } & \text { Time Name } & \text { Code }\end{array}$

10.84

$2 \quad 0.99$ fluoride

$3 \quad 1.54$ chloride

$4 \quad 1.85$ nitrite

52.84 bromide

63.11 nitrate

74.61 phosphate

86.13 sulfate

98.00 oxalate

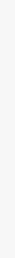

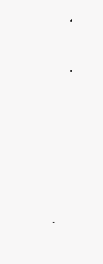

Totals
0.000

20.921

$-1.152$

3.568

11.711

2081.564

226.790

$-13.049$

39.725
114

1977

28

53

206

36109

1467

29

341
473

8257

104

232

1153

354281

16637

419

6258
2

$2-5.40$

$1-4.94$

$1-5.44$

10.00

$1-0.11$

$1-6.49$

$1-4.17$

$1-5.66$

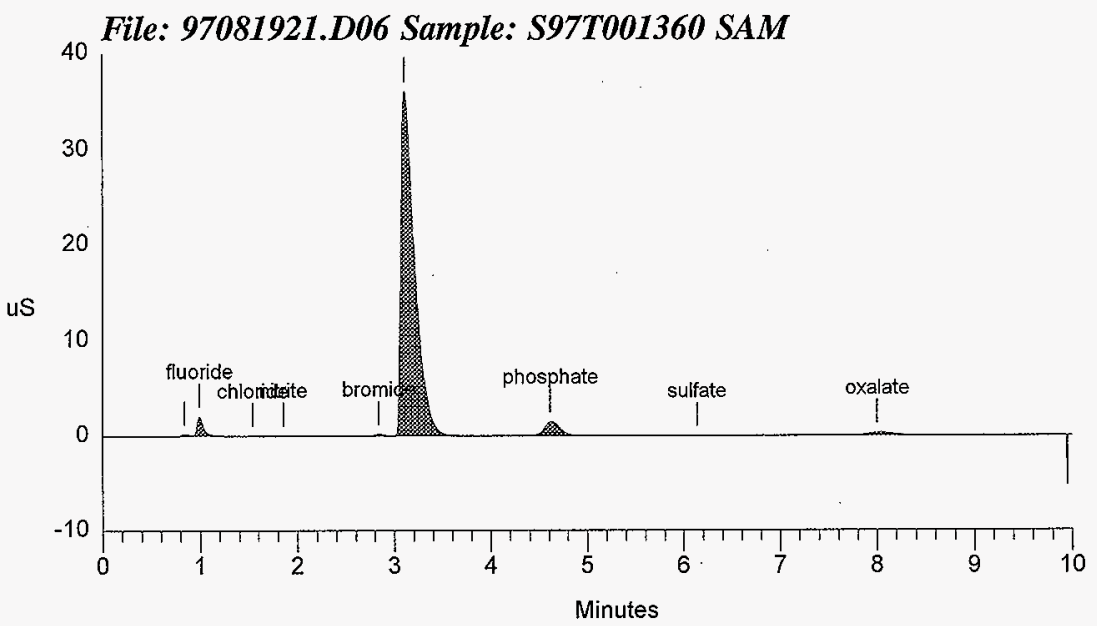


Data Reprocessed On 08/22/1997 10:17:33

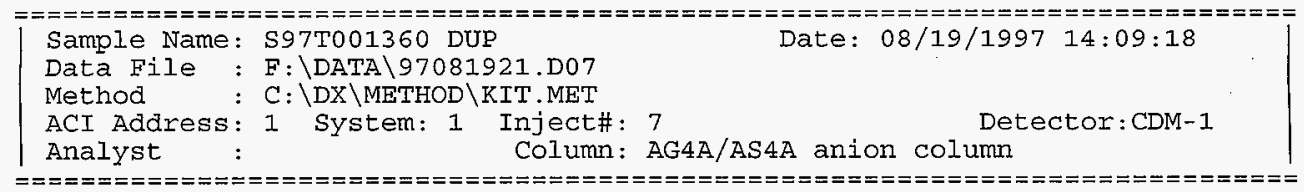

Calibration Volume Dilution Points Rate Start stop Area Reject

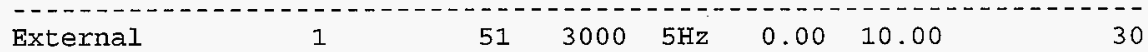

$\star * * * * * * * * * * * * * * * * * * * * * * * * * *$ Peak Report: All Peaks $* * * * * * * * * * * * * * * * * * * * * * * * * * * *$

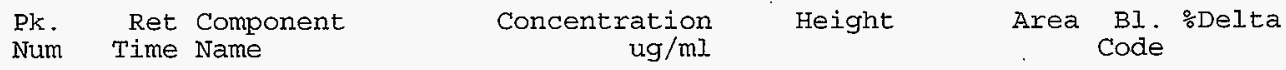

1

0.84

0.99 fluoride

1.54 chloride

1.85 nitrite

2.84 bromide

3.13 nitrate

4.61 phosphate

6.08 sulfate

8.00 oxalate

.

0.000

30.645

$-0.465$

3.773

11.306

1635.185

322.547

$-10.914$

39.700

Totals
102

2856

65

61

199

29359

2129

60

342
412

12232

283

269

1106

271996

23890

829

6254

35171

317273

\section{File: 97081921.D07 Sample: S97T001360 DUP}

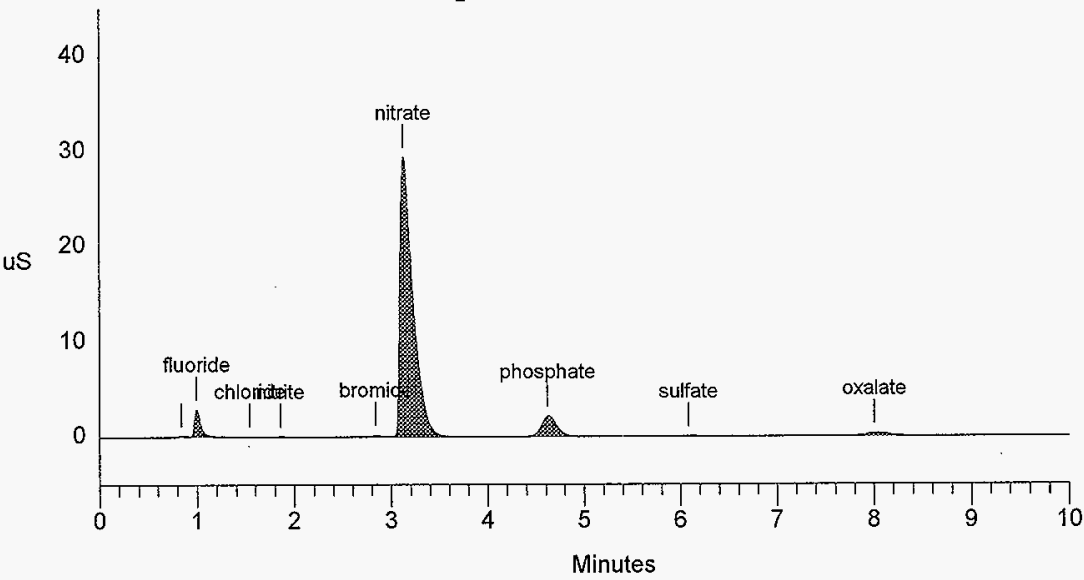


Data Reprocessed On 08/22/1997 10:19:18

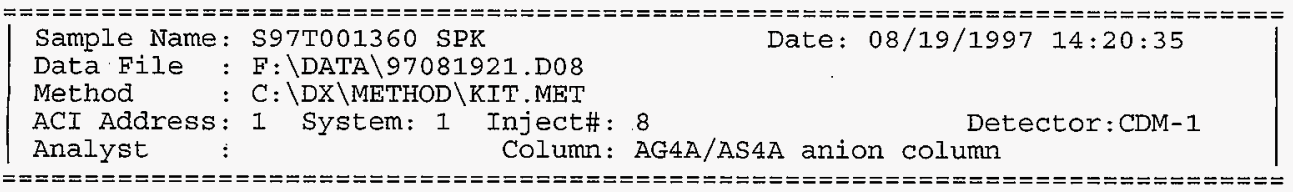

Calibration Volume Dilution Points Rate start stop Area Reject

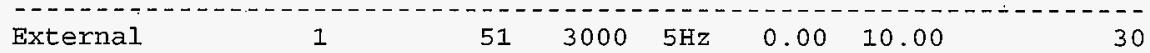

$* * * * * * * * * * * * * * * * * * * * * * * * * * *$ Peak Report: All Peaks $* * * * * * * * * * * * * * * * * * * * * * * * * * * *$

Pk. Ret Component Concentration Height Area Bl. $\%$ Delta Num Time Name $\mathrm{ug} / \mathrm{ml}$

$1 \quad 0.84$

$2 \quad 0.99$

31.53

1.85 nitrite

2.82 bromide

3.10 nitrate

4.61 phosphate

6.03 sulfate

7.95 oxalate

$\begin{array}{rr}0.000 & 201 \\ 46.910 & 4546 \\ 35.743 & 2203 \\ 252.720 & 8968 \\ 279.222 & 5860 \\ 2195.697 & 37743 \\ 479.223 & 3286 \\ 290.303 & 4189 \\ 287.639 & 2406\end{array}$

Totals

3867.457

69403
Code

$\begin{array}{rrr}869 & 2 & \\ 18883 & 2 & -5.40 \\ 9838 & 1 & -5.35 \\ 45661 & 1 & -5.44 \\ 32901 & 1 & 0.00 \\ 376025 & 1 & 0.00 \\ 36031 & 1 & -6.49 \\ 60300 & 1 & -5.83 \\ 44791 & 1 & -6.29\end{array}$

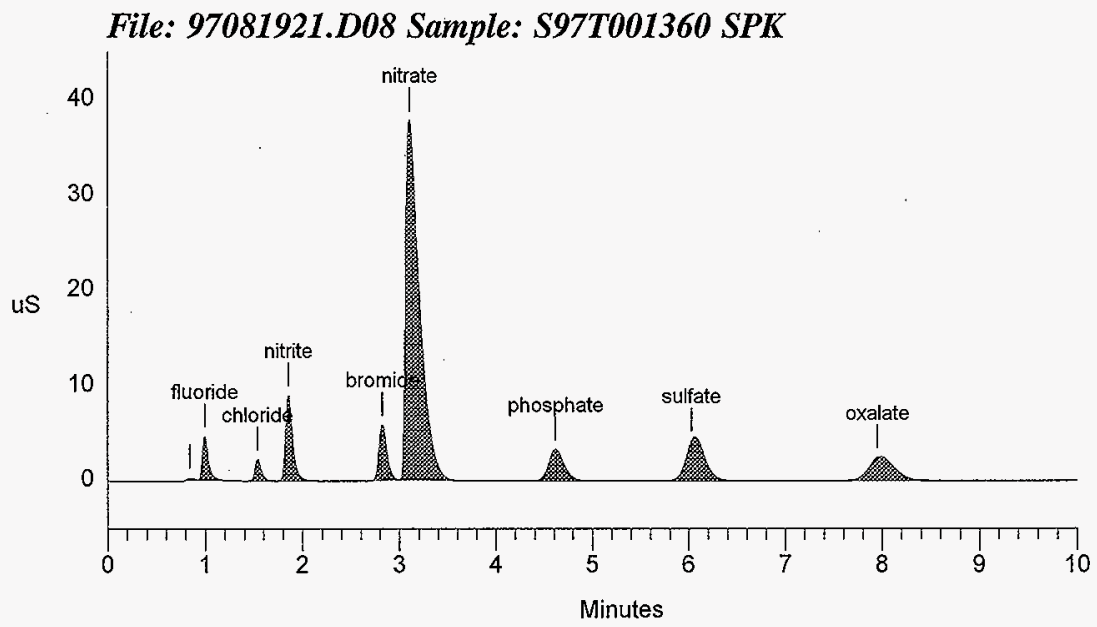


Data Reprocessed On 08/22/1997 10:20:46

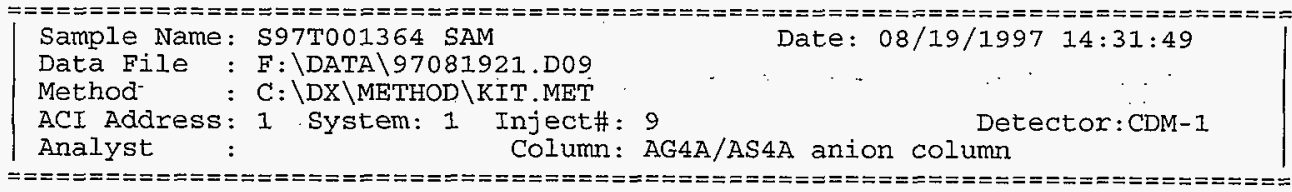

Calibration Volume Dilution points Rate start stop Area Reject

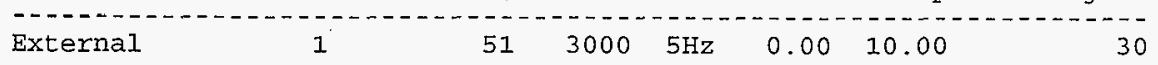

$* * * * * * * * * * * * * * * * * * * * * * * * * * *$ Peak Report: All Peaks $* * * * * * * * * * * * * * * * * * * * * * * * * * * *$

Pk. Ret Component $\quad$ Concentration
Num Time Name Height $/ \mathrm{ml}$

Num Time Name $\mathrm{ug} / \mathrm{ml}$ Code

$\begin{array}{lll}1 & 0.84 & \\ 2 & 0.99 & \text { fluoride } \\ 3 & 1.53 & \text { chloride } \\ 4 & 1.85 & \text { nitrite } \\ 5 & 2.85 & \text { bromide } \\ 6 & 3.11 & \text { nitrate } \\ 7 & 4.61 & \text { phosphate } \\ 8 & 6.08 \text { sulfate } \\ 9 & 8.00 \text { oxalate }\end{array}$

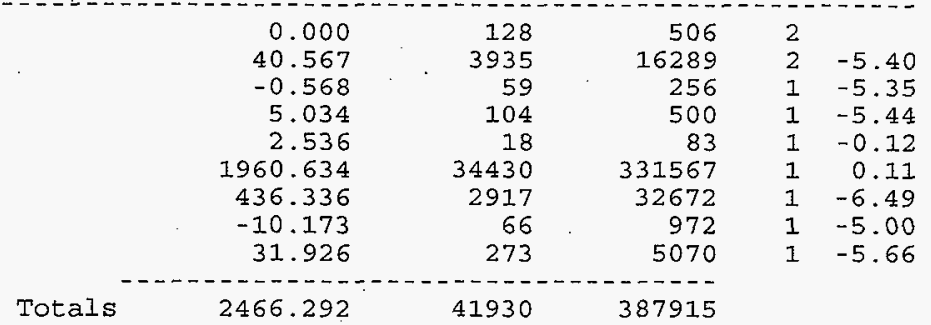

File: 97081921.D09 Sample: S97T001364 SAM

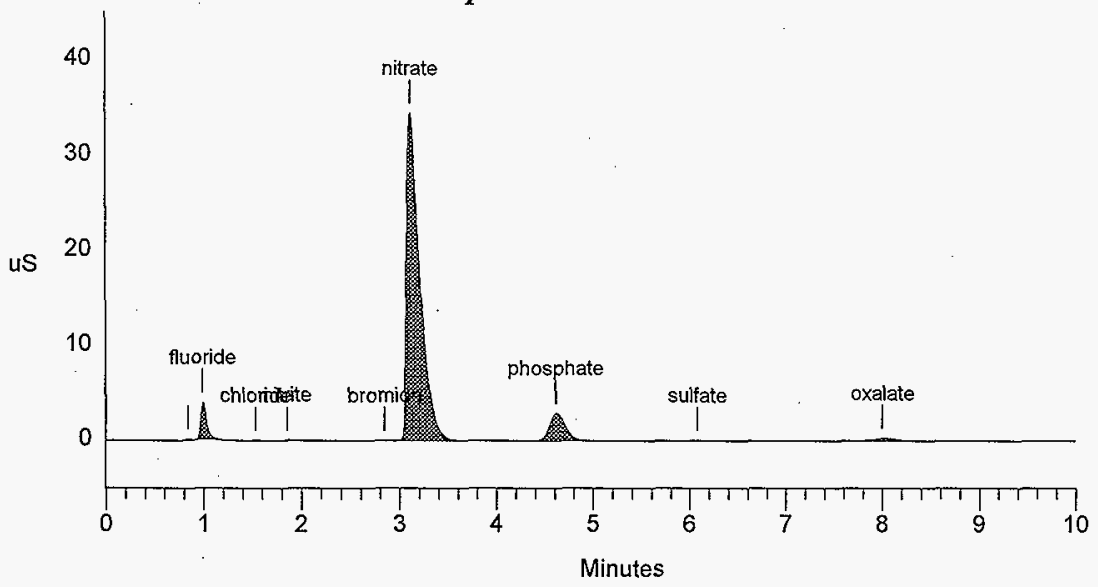


Data Reprocessed on 08/22/1997 10:22:21

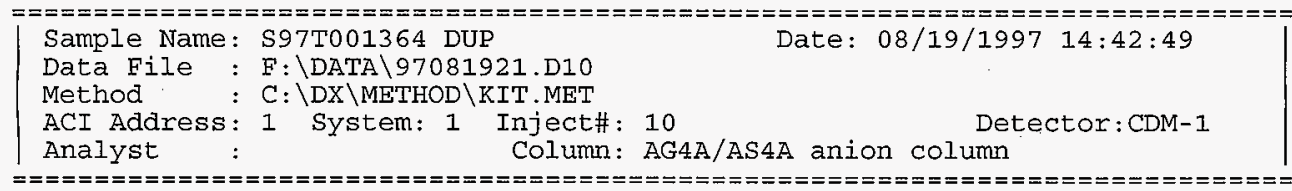

Calibration Volume Dilution Points Rate Start Stop Area Reject

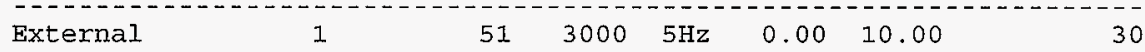

$* * * * * * * * * * * * * * * * * * * * * * * * * * *$ Peak Report: All Peaks $* * * * * * * * * * * * * * * * * * * * * * * * * * * *$

$\begin{array}{lrrr}\text { Pk. Ret Component } & \text { Concentration } & \text { Height } & \text { Area Bl. } \\ \text { Num } & \text { Time Name } & \text { Code }\end{array}$ $\mathrm{ug} / \mathrm{ml}$
0.84
0.99
fluoride
1.53 chloride
1.85 nitrite
2.83 bromide
3.11 nitrate
4.61 phosphate
6.08 sulfate
8.00
oxalate

0.000

45.486

$-0.023$

4.830

2.527

1910.476

477.828

$-11.875$

33.369

141

4374

95

99

18

33441

3248

48

290

Totals $2462.618 \quad 41755 \quad 383946$

$\begin{array}{rrr}607 & 2 & \\ 18300 & 2 & -5.40 \\ 399 & 1 & -5.35 \\ 463 & 1 & -5.44 \\ 82 & 1 & 0.12 \\ 322241 & 1 & -0.11 \\ 35921 & 1 & -6.49 \\ 644 & 1 & -5.00 \\ 5289 & 1 & -5.66\end{array}$

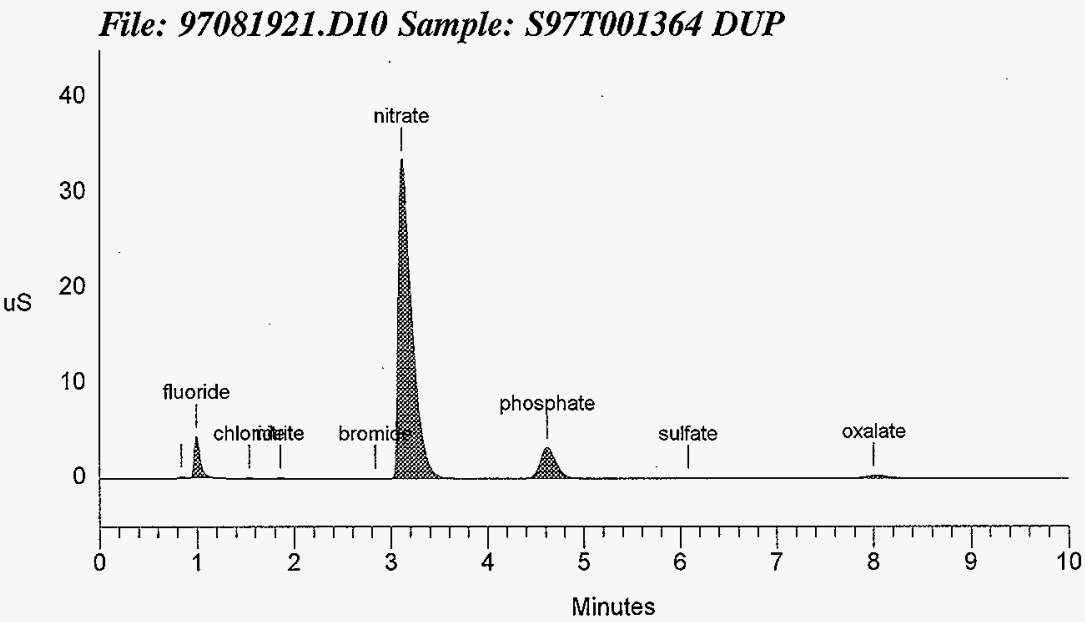


Data Reprocessed On 08/22/1997 10:23:44

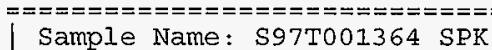

Data File : F: \DATA $\ 97081921 . D 11$

Method : C: \DX\METHOD KKIT.MET

ACI Address: 1 System: 1 Inject\#: 11 Detector:CDM-1

Analyst : Column: AG4A/AS4A anion column

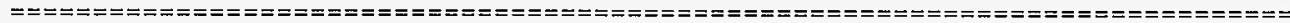

Calibration Volume Dilution Points Rate start stop Area Reject

External $\quad 513000 \quad 5 \mathrm{~Hz} 0.0010 .00 \quad 30$

$* * * * * * * * * * * * * * * * * * * * * * * * * * *$ Peak Report: All Peaks $* * * * * * * * * * * * * * * * * * * * * * * * * * * *$

$\begin{array}{lrrr}\text { Pk. Ret Component } & \text { Concentration } & \text { Height } & \text { Area Bl. } \\ \text { Num } & \text { Time Name } & \text { Code }\end{array}$

10.84

20.99

luoride

0.000

72.442

37.975

267.422

1.85 nitrite

2.82 bromide

3.09 nitrate

286.321

4.59 phosphate

2336.787

750.141

305.224

296.035

Date: $08 / 19 / 199714: 53: 52$

6.03 sulfate

7.95 oxalate

\begin{tabular}{|c|c|c|}
\hline Totals & 4352.346 & 77032 \\
\hline
\end{tabular}

File: 97081921.D11 Sample: S97T001364 SPK

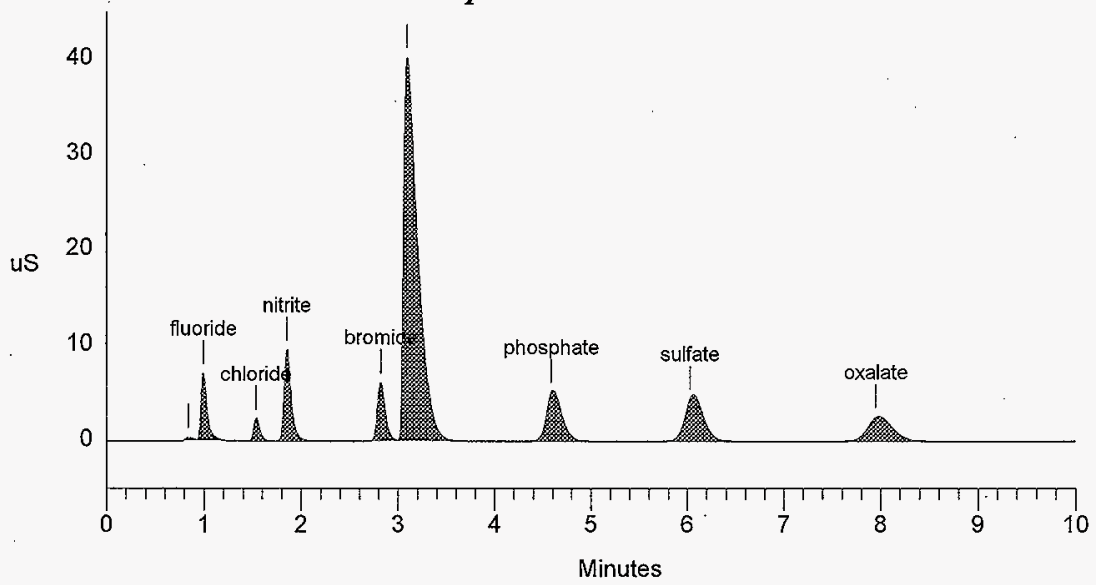




\section{LABCORE Completed Worklist Report for Worklist\# 19380}

Analyst: smf

Instrument: IC02

Book\# 83 32204

Method: LAes $33-100 \mathrm{Rev} / \mathrm{Mod} D-1$

Worklist Comment: BY-101 IC. RCJ

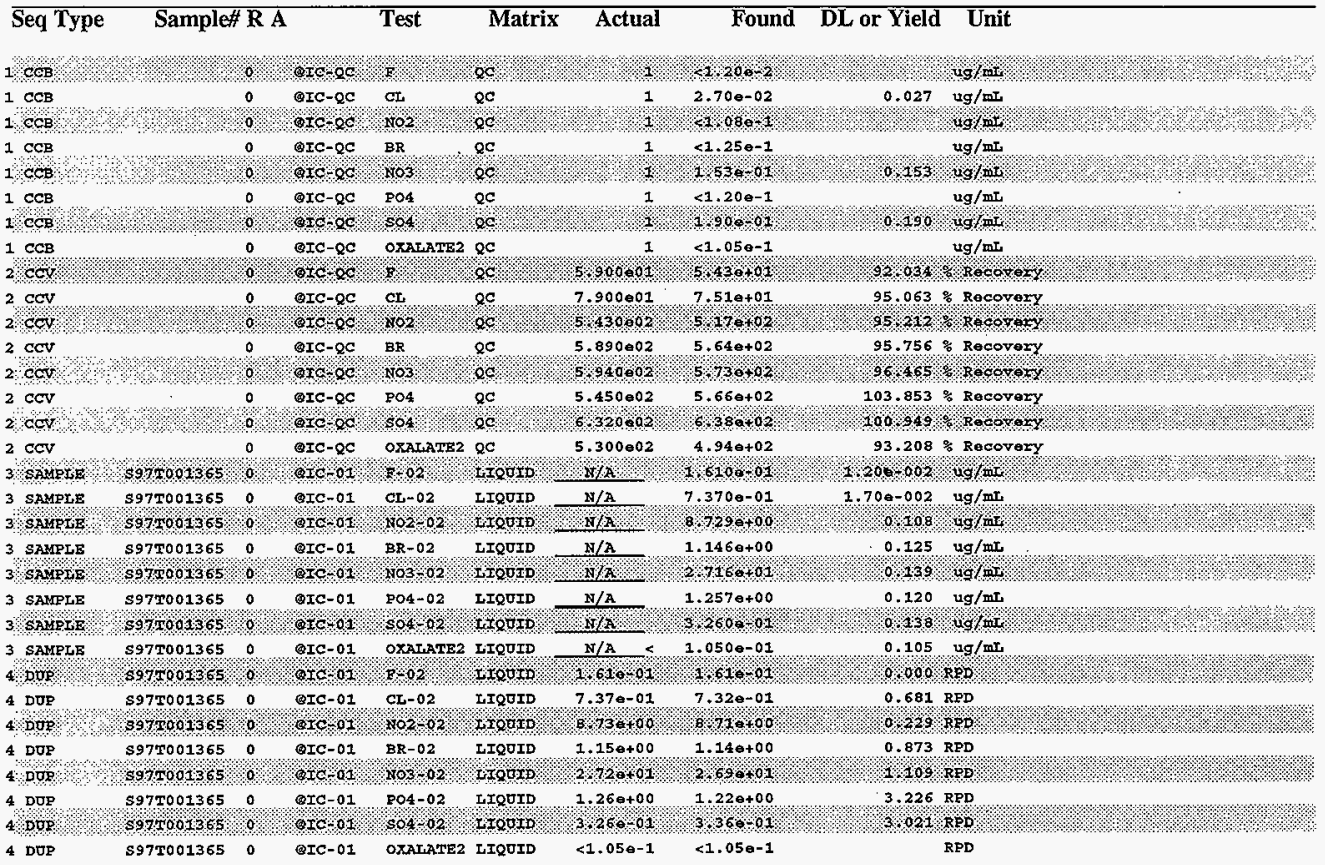

\section{Final page for worklist\# 19380}

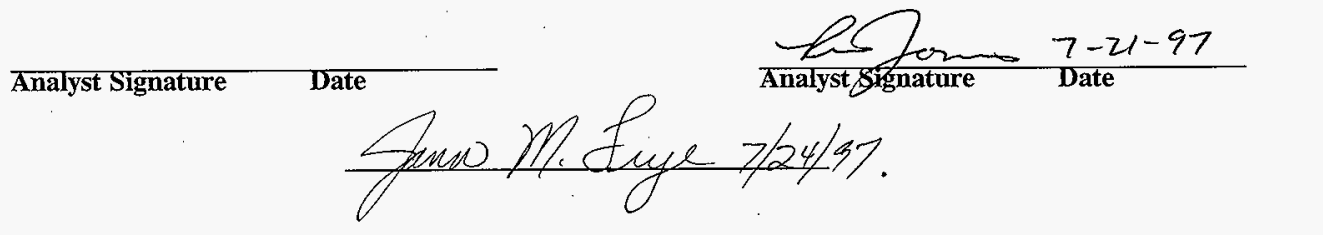

Units shown for $Q C(B L K / B K G)$ may not reflect the actual units. 


\section{LABCORE Data Entry Template for Worklist\# 19380}

Analyst: $3 M$ Finstrument: IC0 2 Book\# $83 N 20-A$

Method: LA-533-105 Rev/Mod

Worklist Comment: BY-101 IC. RCJ

\begin{tabular}{|c|c|c|c|c|c|c|c|c|}
\hline S Type & Sample\# & $\mathrm{RA}$ & Test & Matrix & Group\# & Project & & \\
\hline $1 \mathrm{CCB}$ & & & $@ I C-Q C$ & $\mathrm{QC}$ & & & & \\
\hline $2 \mathrm{CCV}$ & . & & $@ I C-Q C$ & $Q C$ & & & & . \\
\hline 3 SAMPLE & $\begin{array}{c}\text { S97T001365 } \\
\text { Analytes Reque }\end{array}$ & $\begin{array}{l}0 \\
\text { ested: }\end{array}$ & $\begin{array}{l}\text { @IC-OI } \\
\text { BR-02 } \\
\text { OXALATE2 }\end{array}$ & $\begin{array}{l}\text { LIQUID } \\
\text { CL-02 } \\
\text { 2, } \text { PO4-02 }\end{array}$ & $\begin{array}{l}\quad \begin{array}{l}9700039 \\
\text {, }\end{array}-02 \\
\text { SO4-02 }\end{array}$ & $\begin{array}{c}97 \mathrm{BY}-101 \\
\mathrm{NO} 2-02\end{array}$ & , NO3-02 & , \\
\hline 4 DUP & S97T001365 & 0 & @IC-01 & LIQUID & & & & \\
\hline
\end{tabular}

\section{Final page for worklist \# 19380}

Final page
Analyst Signature Sueton $7-20-97$

Analyst Signature Date

Data Entry Comments:

19380124.54

$19380 J_{2} \psi, \operatorname{csv}$

$S=$ Worklist Slot Number, $R=$ Replicate Number, $A=$ Aliquot Code. 
HNF-SD-WM-DP-258, REV. 0

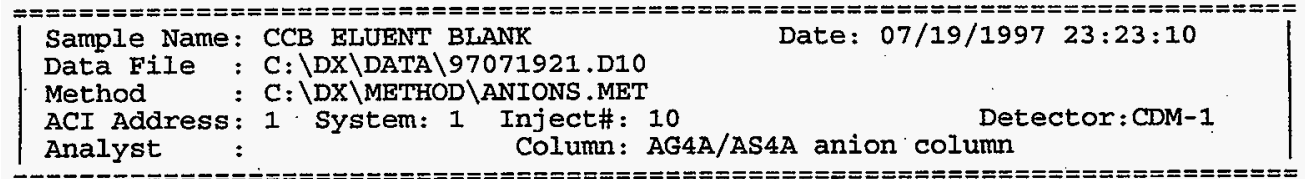

Calibration Volume Dilution Points Rate start stop Area Reject

External $1130005 \mathrm{~Hz} 0.0010 .00 \quad 50$

$\star * * * * * * * * * * * * * * * * * * * * * * * * * *$ Peak Report; All Peaks $* * * * * * * * * * * * * * * * * * * * * * * * * * * *$

Pk. Ret Component $\quad \begin{array}{r}\text { Concentration } \\ \text { ug/ml }\end{array}$
Num. Time Name

\begin{tabular}{rrrrrr} 
& 0.000 & 18 & 106 & 1 & \\
0.000 & 33 & 135 & 1 & \\
0.000 & 123 & 738 & 1 & \\
0.027 & 69 & 229 & 1 & 1.30 \\
& 0.153 & 57 & 404 & 1 & 0.95 \\
& 0.190 & 114 & 1916 & 1 & 2.74 \\
\hline & 0.370 & 413 & 3529 & &
\end{tabular}

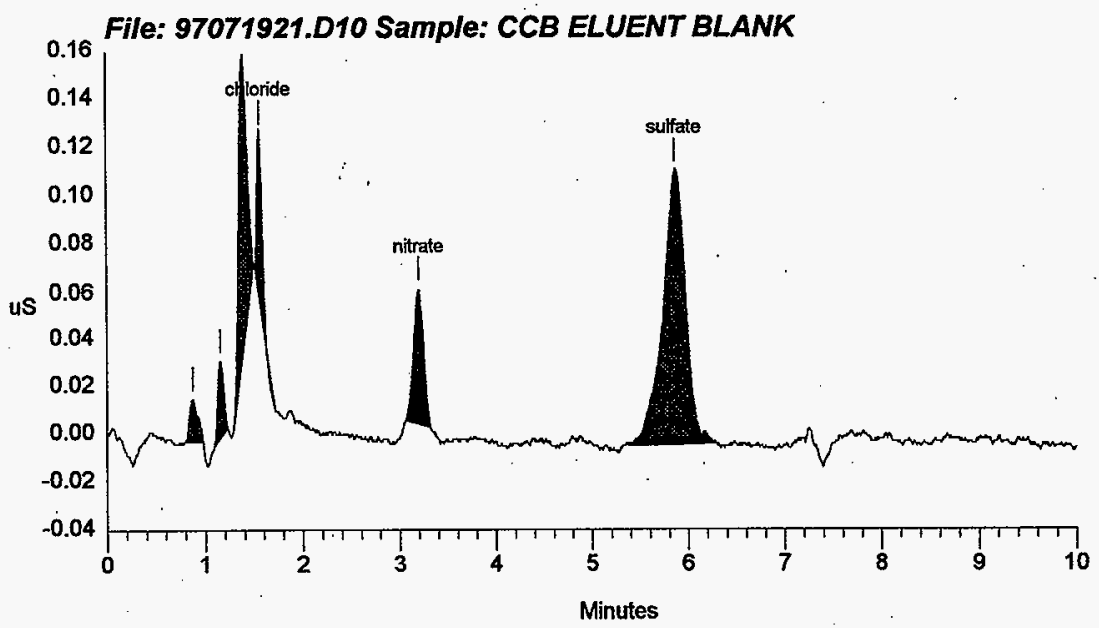


Sample Name: S97T001365

Data File : C:\DX\DATA\97071921.D13

Method : C: $\backslash D X \backslash M E T H O D \backslash A N I O N S$.MET

ACI Address : 1 System: 1 Inject\# : 13

Analyst

Column: AG4A/AS4A anion column

Date: $07 / 20 / 199701: 57: 24$

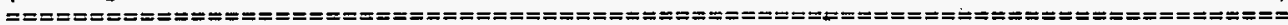

Calibration Volume Dilution Points Rate start stop Area Reject

Calibration

External

$13000 \quad 5 \mathrm{~Hz} \quad 0.00 \quad 10.00$

50

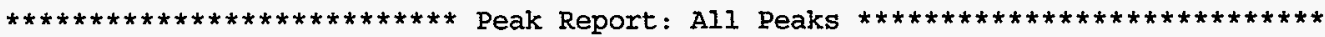

Pk. . Ret Component Concentration Height Area Bl. $\%$ Delta Num Time Name Code

$\begin{array}{lll}1 & 0.10 & \\ 2 & 1.06 & \text { fluoride } \\ 3 & 1.24 & \\ 4 & 1.56 & \text { chloride } \\ 5 & 1.87 & \text { nitrite } \\ 6 & 2.80 & \text { bromide } \\ 7 & 3.09 & \text { nitrate } \\ 8 & 4.51 & \text { phosphate } \\ 9 & 5.87 & \text { sulfate } \\ 10 & 7.68 \text { oxalate }\end{array}$

$\begin{array}{rr}0.000 & 56 \\ 0.161 & 603 \\ 0.000 & 126 \\ 0.737 & 2219 \\ 8.729 & 15715 \\ 1.146 & 1059 \\ 27.163 & 28756 \\ 1.257 & 322 \\ 0.326 & 197 \\ 0.058 & 74\end{array}$

Totals

49127

127

2758

893

10540

86599

6516

244929

4682

3405

1378

$\begin{array}{rr}1 & \\ 2 & 4.95 \\ 2 & \\ 1 & 1.30 \\ 1 & 0.36 \\ 1 & 0.36 \\ 1 & -2.63 \\ 1 & 1.20 \\ 1 & 2.74 \\ 1 & -3.40\end{array}$

361828

File: 97071921.D13 Sample: S97T001365

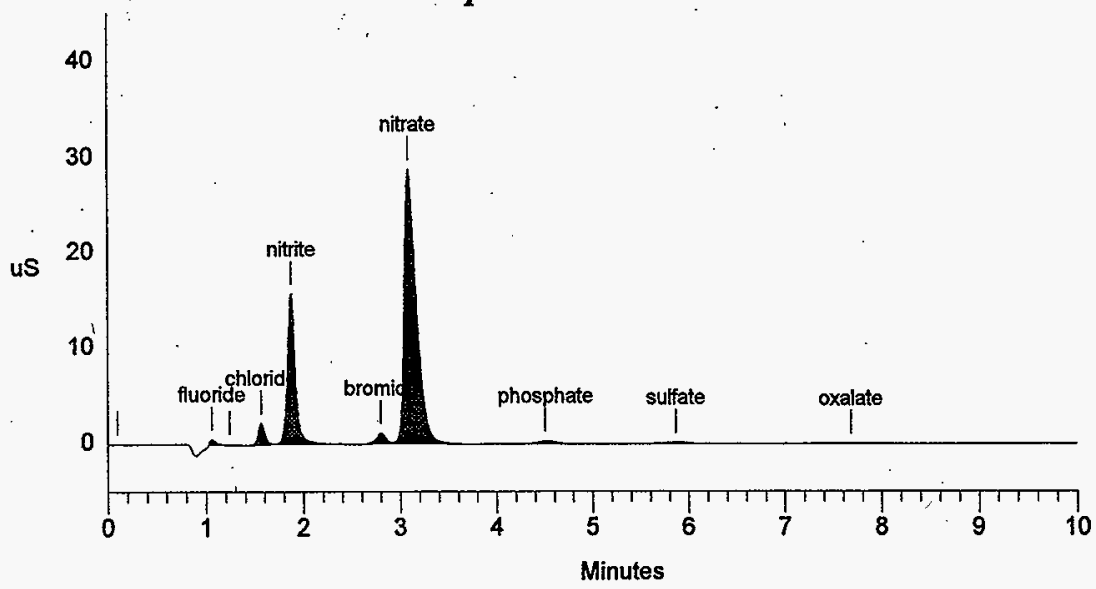


Sample Name: S97T001365 DUP

Data File : C:\DX\DATA $97071921 . D 14$

Method : C: \DX\METHOD \ANIONS.MET

ACI Address: 1 System: 1 Inject\#: 14 Detector:CDM-1

Analyst : $\quad$ Column: AG4A/AS4A anion column

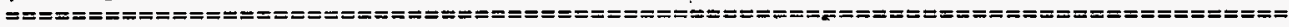

Calibration Volume . Dilution Points Rate start Stop Area Reject

External $1130005 \mathrm{~Hz} 0.0010 .00 \quad 50$

External

Peak Report: All Peaks

Pk. Ret Component

Concentration

Height

Area BI. \&Delta

Num Time Name. $\mathrm{ug} / \mathrm{ml}$ Code

$\begin{array}{rrl}1 & 0.11 & \\ 2 & 1.05 & \text { fluoride } \\ 3 & 1.24 & \\ 4 & 1.56 & \text { chloride } \\ 5 & 1.87 & \text { nitrite } \\ 6 & 2.79 & \text { bromide } \\ 7 & 3.07 & \text { nitrate } \\ 8 & 4.51 & \text { phosphate } \\ 9 & 5.87 & \text { sulfate } \\ 10 & 7.68 \text { oxalate }\end{array}$

0.000

0.161

0.000

0.732

8.705

1.143

26.852

1.215

0.336

0.058

39.202

Totals

49227

1075

28735

325

206

72

$\begin{array}{rr}203 & 1 \\ 2769 & 2 \\ 928 & 2 \\ 10471 & 1 \\ 86352 & 1 \\ 6500 & 1 \\ 41822 & 1 \\ 4516 & 1 \\ 3514 & 1 \\ 1375 & 1\end{array}$

4.29

1.30

0.00

0.12

1375

$-3.05$

1.20

2.74

$1-3.40$

358450

File: 97071921.D14 Sample: S97T001365 DUP

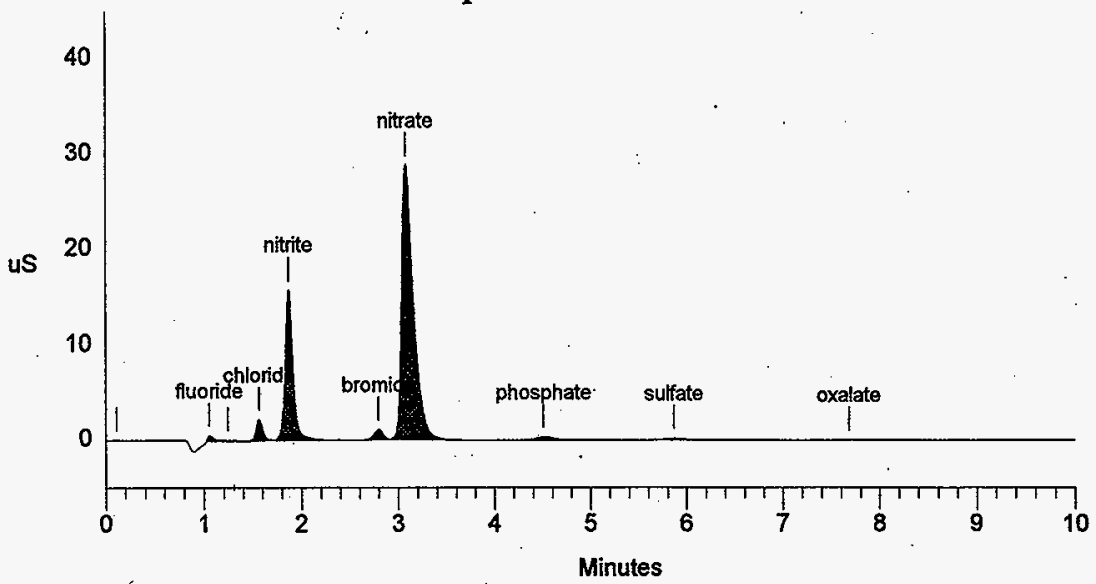

98 


\section{LABCORE Data Entry Template for Worklist\# 18729}

Analyst: B. Gorclet Instrument: ICPOI

Method: LA-505-15T/161 Rev/Mod $G$,

If 6 क्म?

Book\# 626687

Worklist Comment: ICP BY-101 (DIRECT)

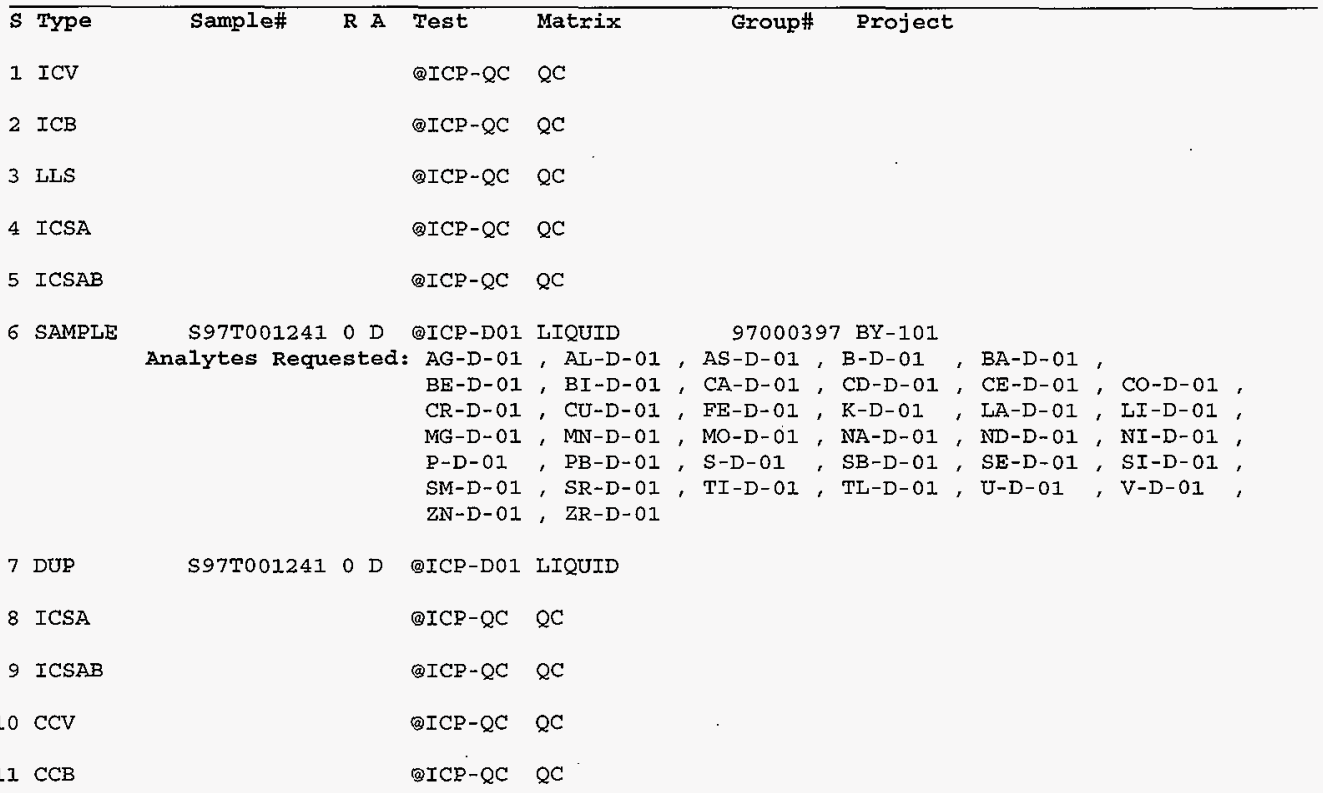

Final page for worklist \# 18729
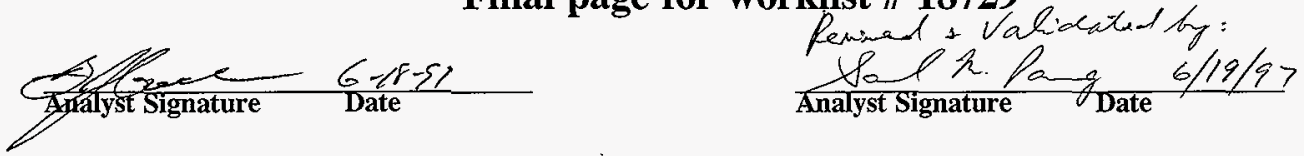

Data Entry Comments:

$\bar{S}=$ Worklist Slot Number, $R=$ Replicate Number, $A=$ Aliquot Code. 
O509 $3722929 \quad$ WESTINGHOUSE $\quad \rightarrow \rightarrow \rightarrow M O-924200 \mathrm{~W}$

001

FCV

FCB

$\angle C S$

ICSA

ICSRT3

S97T001241

$1241-1)$

597001368

1368 - is

CCV

$C C B$

$$
\begin{aligned}
& 597 \pi 001370-6 \quad 4 m 1-6 \ldots 1-2 m 1.8-1 \quad 12.5 \\
& 1320 \quad 401-6 n 1 \quad 2.5 \\
& \text { G1300.D 4ne-6n' } 2.5 \\
& 1370.54 \text { 4nc-6ur } \\
& 1370-x \cdot 4 \pi-6 m / \cdot-1 m+9 n \\
& 1320.9 x
\end{aligned}
$$

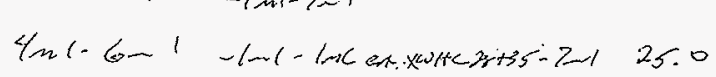

ICSA

ICSAB

$C C V$

$\angle C B$
$6-15-57$

$5=106 \quad 592500330$

By-109 592700136

by- 101 S97toor24. 
Analysis Report

Summary
HNF-SD-WM-DP-258, REV. 0

Hed 06-18-97 01:13:51 PM page 1

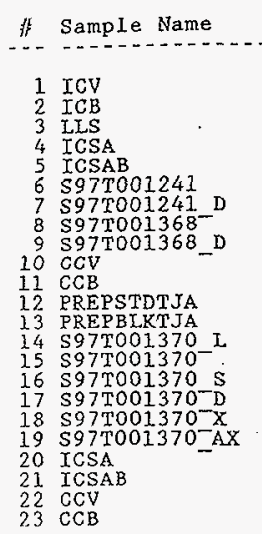

\begin{tabular}{|c|c|c|c|c|c|c|}
\hline File & Method & Date & Time & OpID & Type & Mode \\
\hline $\begin{array}{l}970618 \mathrm{~B} \\
970618 \mathrm{~B} \\
970618 \mathrm{~B} \\
970618 \mathrm{~B} \\
970618 \mathrm{~B} \\
970618 \mathrm{~B} \\
970618 \mathrm{~B} \\
970618 \mathrm{~B} \\
970618 \mathrm{~B} \\
970618 \mathrm{~B} \\
970618 \mathrm{~B} \\
970618 \mathrm{~B} \\
970618 \mathrm{~B} \\
970618 \mathrm{~B} \\
970618 \mathrm{~B} \\
970618 \mathrm{~B} \\
970618 \mathrm{~B} \\
970618 \mathrm{~B} \\
970618 \mathrm{~B} \\
970618 \mathrm{~B} \\
970618 \mathrm{~B} \\
970618 \mathrm{~B}\end{array}$ & $\begin{array}{l}\text { ICP2 } \\
\text { ICP2 } \\
\text { ICP2 } \\
\text { ICP2 } \\
\text { ICP2 } \\
\text { ICP2 } \\
\text { ICP2 } \\
\text { ICP2 } \\
\text { ICP2 } \\
\text { ICP2 } \\
\text { ICP2 } \\
\text { ICP2 } \\
\text { ICP2 } \\
\text { ICP2 } \\
\text { ICP2 } \\
\text { ICP2 } \\
\text { ICP2 } \\
\text { ICP2 } \\
\text { ICP2 } \\
\text { ICP2 } \\
\text { ICP2 } \\
\text { ICP2 } \\
\text { ICP2 }\end{array}$ & $\begin{array}{l}06 / 18 / 97 \\
06 / 18 / 97 \\
06 / 18 / 97 \\
06 / 18 / 97 \\
06 / 18 / 97 \\
06 / 18 / 97 \\
06 / 18 / 97 \\
06 / 18 / 97 \\
06 / 18 / 97 \\
06 / 18 / 97 \\
06 / 18 / 97 \\
06 / 18 / 97 \\
06 / 18 / 97 \\
06 / 18 / 97 \\
06 / 18 / 97 \\
06 / 18 / 97 \\
06 / 18 / 97 \\
06 / 18 / 97 \\
06 / 18 / 97 \\
06 / 18 / 97 \\
06 / 18 / 97 \\
06 / 18 / 97 \\
06 / 18 / 97\end{array}$ & $\begin{array}{l}10: 16 \\
10: 19 \\
10: 22 \\
10: 25 \\
10: 29 \\
10: 33 \\
10: 36 \\
10: 39 \\
10: 43 \\
10: 47 \\
10: 50 \\
10: 55 \\
10: 59 \\
11: 06 \\
11: 09 \\
11: 12 \\
11: 15 \\
11: 19 \\
11: 22 \\
11: 25 \\
11: 28 \\
11: 31 \\
11: 36\end{array}$ & $\begin{array}{l}\text { BJG } \\
\text { BJGG } \\
\text { BJGG } \\
\text { BJG } \\
\text { BJG } \\
\text { BJG } \\
\text { BJG } \\
\text { BJG } \\
\text { BJG } \\
\text { BJG } \\
\text { BJG } \\
\text { BJG } \\
\text { BJG } \\
\text { BJG } \\
\text { BJG } \\
\text { BJG } \\
\text { BJG } \\
\text { BJG } \\
\text { BJG } \\
\text { BJG } \\
\text { BJG } \\
\text { BJG } \\
\text { BJG }\end{array}$ & $\begin{array}{l}Q \\
Q \\
Q \\
0 \\
0 \\
Q \\
S \\
S \\
S \\
S \\
Q \\
Q \\
Q \\
Q \\
S \\
S \\
S \\
S \\
S \\
S \\
Q \\
Q \\
Q \\
Q\end{array}$ & $\begin{array}{l}\text { GONC } \\
\text { CONC } \\
\text { CONC } \\
\text { CONC } \\
\text { CONC. } \\
\text { CONC } \\
\text { GONG } \\
\text { CONC } \\
\text { CONC } \\
\text { CONC } \\
\text { CONC } \\
\text { CONG } \\
\text { CONC } \\
\text { CONC } \\
\text { CONC } \\
\text { CONC } \\
\text { CONC } \\
\text { CONC } \\
\text { CONG } \\
\text { CONC } \\
\text { CONC } \\
\text { CONC } \\
\text { CONG }\end{array}$ \\
\hline
\end{tabular}

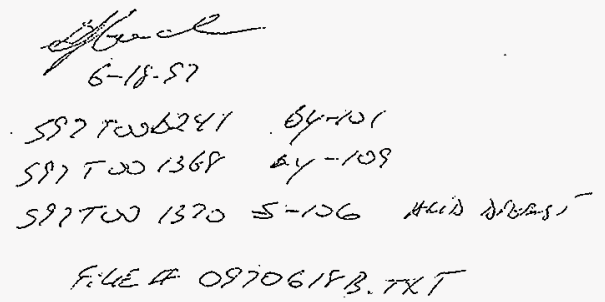

SIGNATURE ABOVE REPRESENTS CHEMICAL TECHNOLOGIST/CHEMIST THAT COMPLETED VERIFIED THE CALIBRATIONANALYSIS ON PAGES $10 /$ TO 105 . 


\begin{tabular}{|c|c|c|c|c|c|c|c|}
\hline 湆 & Sample Name & $\mathrm{Ag}$ & Al. & As & $\begin{array}{l}B \\
------\end{array}$ & $\mathrm{Ba}$ & $\mathrm{Be}$ \\
\hline $\begin{array}{l}1 \\
2 \\
3 \\
4 \\
5 \\
6 \\
7 \\
8 \\
9 \\
10 \\
11 \\
12 \\
13 \\
14 \\
15 \\
16 \\
17 \\
18 \\
19 \\
20 \\
21 \\
22 \\
23\end{array}$ & $\begin{array}{l}\text { ICV } \\
\text { ICB } \\
\text { ILS } \\
\text { ICSA } \\
\text { ICSAB } \\
\text { S97T001241 } \\
\text { S97T001241_D } \\
\text { S97T001368- } \\
\text { S97T001368_D } \\
\text { CCV } \\
\text { CCB } \\
\text { PREPSTDT TA } \\
\text { PREPBLKTJA } \\
\text { S97T001370_L } \\
\text { S97T001370- } \\
\text { S97T001370-S } \\
\text { S97T001370-D } \\
\text { S97T001370-X } \\
\text { S97T001370-AX } \\
\text { ICSA } \\
\text { ICSAB } \\
\text { CCY } \\
\text { CCB }\end{array}$ & $\begin{array}{l}4.900 \\
-.0007 \\
.0207 \\
.0003 \\
.9654 \\
-.0358 \\
-.0163 \\
-.0549 \\
-.0547 \\
4.860 \\
-.0016 \\
.9740 \\
-.0007 \\
.0613 \\
.0731 \\
.0727 \\
.0724 \\
.0158 \\
191.0 \\
1067 \\
1.004 \\
4.830 \\
Q .0289\end{array}$ & $\begin{array}{l}4.892 \\
-.0026 \\
.1066 \\
245.9 \\
244.1 \\
-.0452 \\
.0847 \\
-.0953 \\
-.3220 \\
4.824 \\
-.0045 \\
4.810 \\
0.0606 \\
58.80 \\
57.90 \\
55.75 \\
56.67 \\
58.58 \\
300.0 \\
240.5 \\
240.2 \\
4.833 \\
-.0137\end{array}$ & $\begin{array}{l}5.207 \\
-.0100 \\
.2052 \\
-.0250 \\
-.0504 \\
-.1148 \\
.0163 \\
-.0772 \\
-.1846 \\
5.115 \\
.0062 \\
5.047 \\
-.0101 \\
-.0322 \\
.0327 \\
-.0214 \\
.0015 \\
-.3443 \\
250.7 \\
. .0498 \\
-.0127 \\
5.141 \\
-.0076\end{array}$ & $\begin{array}{l}5.061 \\
.0035 \\
.1047 \\
-.0071 \\
-.0080 \\
.0600 \\
-.0098 \\
-.0496 \\
.0302 \\
4.976 \\
.0005 \\
4.978 \\
0.1709 \\
.7370 \\
.6646 \\
.5096 \\
.3067 \\
.6747 \\
247.1 \\
.0046 \\
.0036 \\
4.999 \\
-.0005\end{array}$ & $\begin{array}{l}4.969 \\
.0001 \\
.1017 \\
.0001 \\
.4701 \\
.0628 \\
.0628 \\
.0580 \\
.0581 \\
4.835 \\
.0001 \\
4.730 \\
.0011 \\
.0315 \\
.0315 \\
.0288 \\
.0302 \\
.0316 \\
246.7 \\
.0003 \\
4856 \\
4.885 \\
.0001\end{array}$ & $\begin{array}{l}5.120 \\
.0001 \\
.0110 \\
.0005 \\
.4875 \\
.0043 \\
.0043 \\
.0052 \\
.0009 \\
5.050 \\
.0004 \\
5.088 \\
.0002 \\
.0017 \\
.0029 \\
.0011 \\
.0020 \\
.0110 \\
2480 \\
.0006 \\
.4776 \\
5.057 \\
. .0000\end{array}$ \\
\hline 据 & Sample Name & $\mathrm{Bi}$ & $\mathrm{Ga}$ & cd & $\mathrm{Ce}$ & Co & $\mathrm{Cr}$ \\
\hline $\begin{array}{l}1 \\
2 \\
3 \\
4 \\
5 \\
6 \\
7 \\
8 \\
9 \\
10 \\
11 \\
12 \\
13 \\
14 \\
15 \\
16 \\
17 \\
18 \\
19 \\
20 \\
21 \\
22 \\
23\end{array}$ & $\begin{array}{l}\text { ICV } \\
\text { ICB } \\
\text { LLS } \\
\text { ICSA } \\
\text { ICSAB } \\
\text { S97T001241 } \\
\text { S97T001241_D } \\
\text { S97T001368- } \\
\text { S97T001368_D } \\
\text { GCV } \\
\text { CCB } \\
\text { PREPSTDTJA } \\
\text { PREPBLKTJA } \\
\text { S97T001370_L } \\
\text { S97T001370- } \\
\text { S97TOO1370-S } \\
\text { S97T001370-D } \\
\text { S97T001370-X } \\
\text { S97T001370-AX } \\
\text { ICSA } \\
\text { ICSAB } \\
\text { CCV } \\
\text { CCB }\end{array}$ & $\begin{array}{l}5.072 \\
.0048 \\
.1943 \\
-.0167 \\
-.0261 \\
-.0725 \\
-.1889 \\
-.3630 \\
-.4296 \\
4.992 \\
.0076 \\
4.837 \\
-.0358 \\
.8627 \\
.9047 \\
.8860 \\
.8869 \\
.3545 \\
251.6 \\
-.0282 \\
-.0344 \\
4.979 \\
.0219\end{array}$ & $\begin{array}{l}4.917 \\
.0001 \\
2137 \\
249.8 \\
253.7 \\
.4011 \\
.4264 \\
.0137 \\
.0387 \\
5.003 \\
-.0050 \\
4.864 \\
.0502 \\
9022 \\
1.011 \\
1.056 \\
1.152 \\
.6903 \\
252.4 \\
249.3 \\
248.4 \\
4.930 \\
-.0007\end{array}$ & $\begin{array}{l}4.993 \\
.0000 \\
.0097 \\
.0009 \\
.9401 \\
-.0081 \\
.0028 \\
.0119 \\
.0098 \\
4.960 \\
.0015 \\
4.760 \\
.0004 \\
.0885 \\
.0899 \\
.0762 \\
.0808 \\
.0795 \\
245.6 \\
.0028 \\
.9215 \\
4.907 \\
. .0010\end{array}$ & $\begin{array}{l}4.937 \\
.0011 \\
.2063 \\
.0030 \\
.0071 \\
.0603 \\
.1017 \\
.1667 \\
.0584 \\
4.807 \\
.0077 \\
4.867 \\
.0017 \\
.0109 \\
.0277 \\
.0202 \\
.0210 \\
.05570 \\
246.3 \\
.0065 \\
.0044 \\
4.810 \\
.0008\end{array}$ & $\begin{array}{l}5.071 \\
.0000 \\
.0399 \\
.0004 \\
.4718 \\
-.0156 \\
-.0234 \\
.0163 \\
5.0019 \\
5.054 \\
.0009 \\
4.927 \\
.0005 \\
.0084 \\
.0103 \\
.0146 \\
.0103 \\
.0201 \\
245.0 \\
.0008 \\
.4599 \\
5.011 \\
-.0004\end{array}$ & $\begin{array}{l}4.960 \\
.0000 \\
.0203 \\
-.0035 \\
.4716 \\
-.0086 \\
-.0141 \\
.0189 \\
-.0110 \\
4.949 \\
-.0003 \\
4.801 \\
.0018 \\
29.66 \\
28.90 \\
27.92 \\
28.27 \\
29.51 \\
275.2 \\
-.0028 \\
.4612 \\
4.891 \\
.0004\end{array}$ \\
\hline
\end{tabular}




\section{HNF-SD-WM-DP-258, REV. 0}

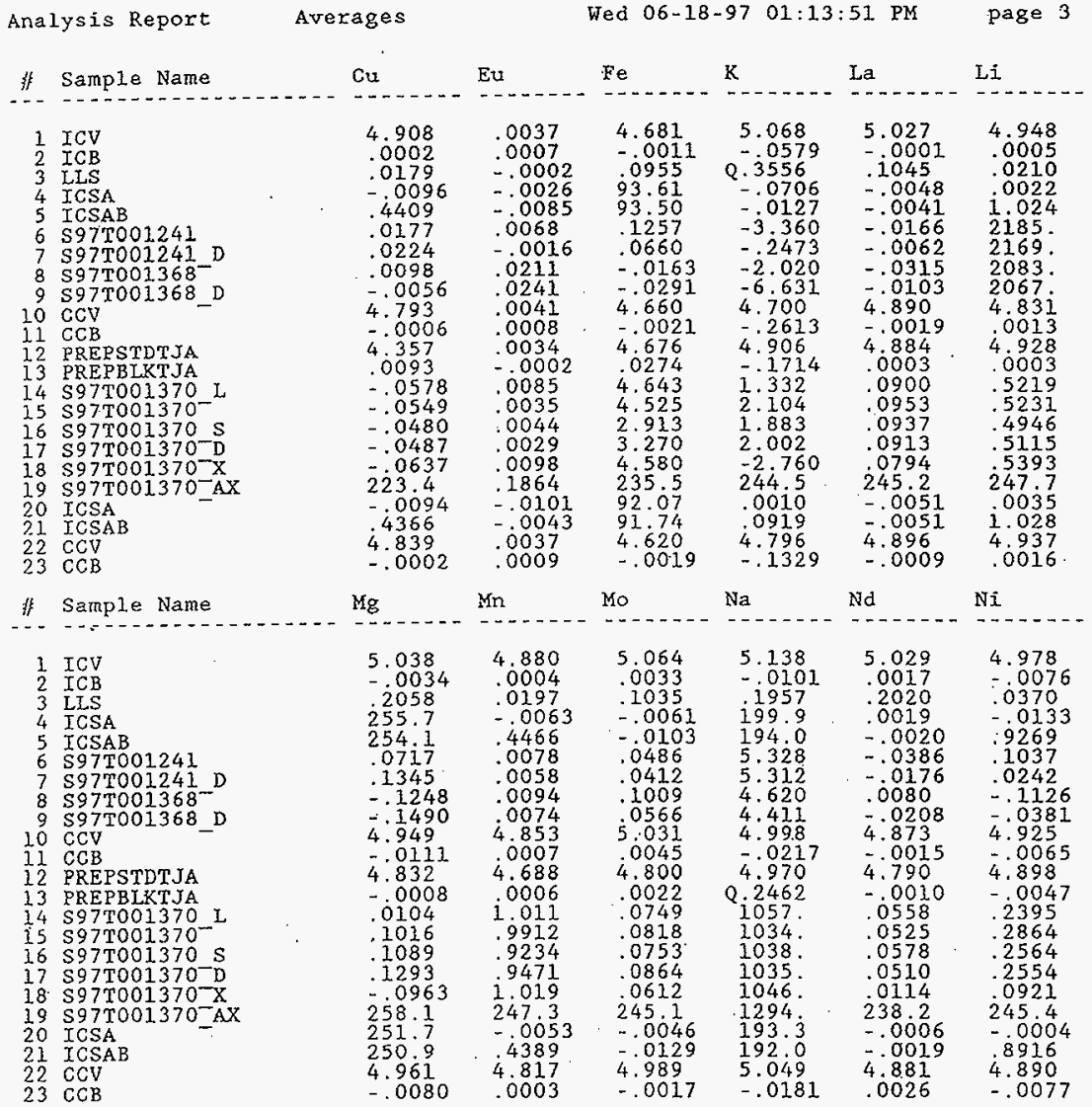




\section{HNF-SD-WPA-DP-258, REV. 0}

Analysis Report

Averages
Wed 06-18-97 01:13:51 PM page 4

\begin{tabular}{|c|c|c|c|c|c|c|c|}
\hline 非 & Sample Name & $P$ & $\mathrm{~Pb}$ & $\mathrm{~s}$ & Sb & $\mathrm{Se}$ & $S i$ \\
\hline $\begin{array}{l}1 \\
2 \\
3 \\
4 \\
5 \\
6 \\
7 \\
8 \\
9 \\
10 \\
11 \\
12 \\
13 \\
14 \\
15 \\
16 \\
17 \\
18 \\
19 \\
20 \\
21 \\
22 \\
23\end{array}$ & $\begin{array}{l}\text { ICV } \\
\text { ICB } \\
\text { LIS } \\
\text { ICSA } \\
\text { ICSAB } \\
\text { S97T001241 } \\
\text { S97T001241_D } \\
\text { S97T001368- } \\
\text { S97T001368_D } \\
\text { CCV } \\
\text { CGB } \\
\text { PREPSTDTJA } \\
\text { PREPBLKTJA } \\
\text { S97TO01370 L } \\
\text { S97TO01370- } \\
\text { S97T001370_S } \\
\text { S97TO01370-D } \\
\text { S97TO01370-X } \\
\text { S97TO01370-AX } \\
\text { ICSA } \\
\text { ICSAB } \\
\text { CCV } \\
\text { CCB }\end{array}$ & $\begin{array}{l}5: 109 \\
-.0023 \\
.4090 \\
.0125 \\
.0060 \\
.1775 \\
.1517 \\
.1560 \\
-.0507 \\
5.028 \\
.0048 \\
4.829 \\
10118 \\
110.6 \\
109.2 \\
103.1 \\
104.2 \\
109.7 \\
354.2 \\
.0337 \\
.0185 \\
4.972 \\
-.0066\end{array}$ & $\begin{array}{l}4.921 \\
.0142 \\
.2199 \\
0390 \\
1.021 \\
.3527 \\
.1893 \\
.4596 \\
.02442 \\
4.925 \\
.0058 \\
4.746 \\
.0312 \\
.1837 \\
.2368 \\
.2146 \\
.2056 \\
.6606 \\
2493 \\
.0233 \\
.9699 \\
4.873 \\
.0000\end{array}$ & $\begin{array}{l}4.989 \\
.0001 \\
.2040 \\
-.0494 \\
-.0294 \\
1.520 \\
1.617 \\
1.347 \\
1.406 \\
4.935 \\
-.0093 \\
4.914 \\
Q .1037 \\
27.24 \\
27.02 \\
27.80 \\
22.47 \\
26.30 \\
269.8 \\
-.0572 \\
-.0413 \\
4.922 \\
.0055\end{array}$ & $\begin{array}{l}4.812 \\
.0028 \\
.0944 \\
.0038 \\
-.0004 \\
-.0057 \\
-.0060 \\
.0130 \\
-.0243 \\
4.760 \\
-.0029 \\
4.792 \\
.0006 \\
.0113 \\
.0096 \\
-.0158 \\
-.0103 \\
-1035 \\
248.6 \\
.0148 \\
.0129 \\
4.763 \\
-.0128\end{array}$ & $\begin{array}{l}4.733 \\
.0069 \\
.1891 \\
.0312 \\
.0110 \\
.5747 \\
.5739 \\
1.230 \\
.9671 \\
4.673 \\
.0336 \\
4.539 \\
.0363 \\
.3034 \\
.0529 \\
.0415 \\
.0580 \\
.7612 \\
240.2 \\
.0387 \\
.0384 \\
4.696 \\
.0176\end{array}$ & $\begin{array}{l}4.813 \\
.0046 \\
.1893 \\
. .0012 \\
.0053 \\
2.108 \\
2.080 \\
1.323 \\
1.299 \\
4.783 \\
.0080 \\
6.694 \\
Q .2065 \\
.7748 \\
.7468 \\
.1722 \\
1.955 \\
.8341 \\
246.8 \\
.0175 \\
.0082 \\
4.757 \\
.0061\end{array}$ \\
\hline$\#$ & Sample Name & $\mathrm{Sm}$ & $\mathrm{Sr}$ & Th & $\mathrm{Ti}$ & $\mathrm{T} 1$ & $U$ \\
\hline $\begin{array}{r}9 \\
10 \\
11 \\
12 \\
13 \\
14 \\
15 \\
16 \\
17 \\
18 \\
19 \\
20 \\
21\end{array}$ & $\begin{array}{l}\text { ICV } \\
\text { ICB } \\
\text { LLS } \\
\text { ICSA } \\
\text { ICSAB } \\
\text { S97T00124I } . \\
\text { S97T001241_D } \\
\text { S97T001368- } \\
\text { S97T001368_D } \\
\text { CCV } \\
\text { CCB } \\
\text { PREPSTDTJA } \\
\text { PREPBLKTJA } \\
\text { S97TO01370-L } \\
\text { S97T001370- } \\
\text { S97T001370-S } \\
\text { S97T001370-D } \\
\text { S97T001370-X } \\
\text { S97T001370-AX } \\
\text { ICSA } \\
\text { ICSAB } \\
\text { CCV } \\
\text { CCB }\end{array}$ & $\begin{array}{l}4.928 \\
.0068 \\
.2014 \\
-.0088 \\
.0036 \\
-.0013 \\
.0431 \\
.2574 \\
3251 \\
4.803 \\
.0100 \\
4.722 \\
-.0008 \\
.0567 \\
.0355 \\
.0406 \\
.0286 \\
.1625 \\
2386 \\
.0016 \\
-.0039 \\
4.826 \\
.0137\end{array}$ & $\begin{array}{l}4.977 \\
.0002 \\
.0208 \\
.0021 \\
.0020 \\
.0082 \\
.0082 \\
.0109 \\
.0108 \\
4.853 \\
.0004 \\
4.739 \\
.0005 \\
.0308 \\
.0287 \\
.0278 \\
.0270 \\
.0319 \\
2444 \\
.0021 \\
.0019 \\
4.858 \\
.0003\end{array}$ & $\begin{array}{l}.1198 \\
-.0016 \\
.0037 \\
-.0125 \\
-.0148 \\
-.1285 \\
-.0068 \\
-.1330 \\
-.2293 \\
.1249 \\
-.0068 \\
.1172 \\
.0002 \\
.0238 \\
.0565 \\
.0486 \\
.0537 \\
-.0462 \\
6.263 \\
-.0063 \\
-.0078 \\
.1299 \\
-.0012\end{array}$ & $\begin{array}{l}4.958 \\
.0002 \\
.0209 \\
.0001 \\
.0009 \\
.0039 \\
.0044 \\
-.0056 \\
-.0154 \\
4.882 \\
-.0000 \\
4.436 \\
.0002 \\
.0168 \\
.0123 \\
.0129 \\
.0117 \\
.0049 \\
245.4 \\
.0004 \\
.0006 \\
4.869 \\
.0000\end{array}$ & $\begin{array}{l}4.766 \\
.0171 \\
.4495 \\
.0447 \\
.0661 \\
.2823 \\
.6403 \\
.9068 \\
.6482 \\
4.728 \\
.0233 \\
4.500 \\
.0257 \\
.1286 \\
.0768 \\
.0690 \\
.0993 \\
.5088 \\
240.3 \\
.0998 \\
.0638 \\
4.635 \\
.0170\end{array}$ & $\begin{array}{l}9.702 \\
.0158 \\
.4747 \\
.0548 \\
.0615 \\
.6290 \\
1.6436 \\
1.605 \\
1.796 \\
9.461 \\
.0307 \\
9.266 \\
9.0101 \\
1.796 \\
1.651 \\
1.631 \\
1.642 \\
1.994 \\
466.8 \\
.0790 \\
.0502 \\
9.499 \\
.0340\end{array}$ \\
\hline
\end{tabular}


Wed 06-18-97 01:13:51 PM page 5

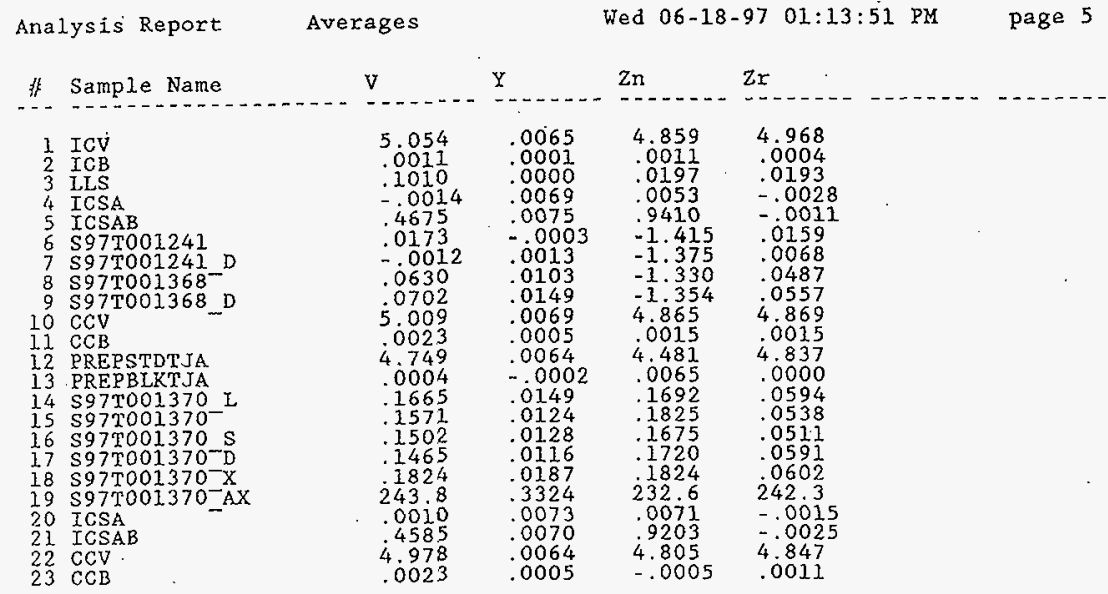




\section{LABCORE Data Entry Template for Worklist\# 19395}

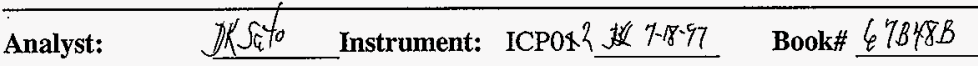

Method: LA-505-15¥/161 Rev/Mod C-1

㛙 7.18 .9

Worklist Comment: ICP BY-101 (DIRECT)

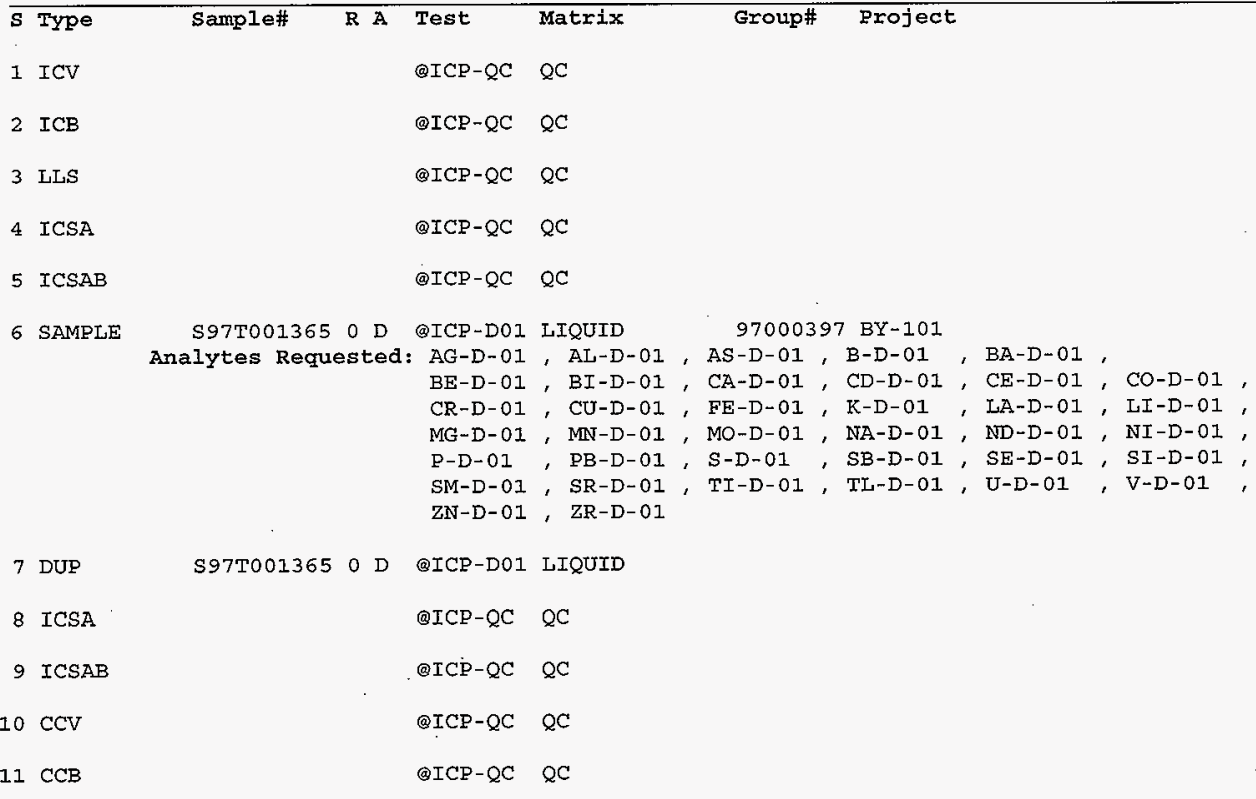

\section{Final page for worklist \# 19395}

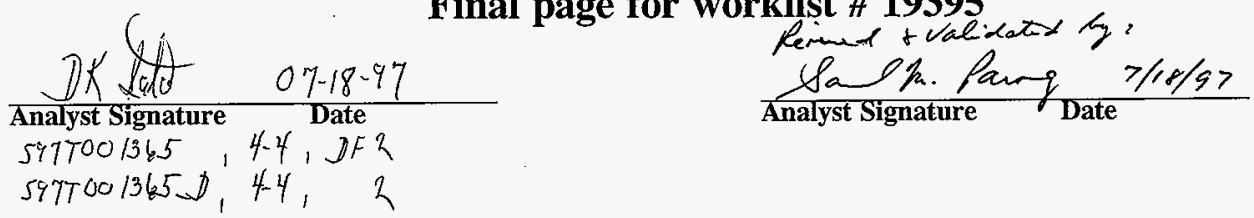

Data Entry Comments:

$\bar{S}=$ Worklist Slot Number, $R=$ Replicate Number, $A=$ Aliquot Code. 
HNF-SD-WM-DP-258, REV. 0

Fri $07-18-9712: 40: 22$ PM page 1

Analysis Report Summary Fri 07-18-97 12:40:22 PM page 1

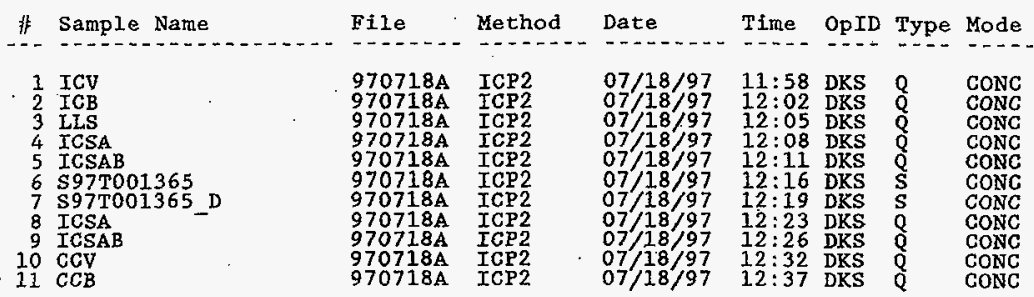

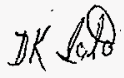

$0 \% \cdot 18 \cdot 97$

5477001365

BY. 101 SIGNATURE ABOVE REPRESENTS CHEMICAL TECHNOLOGIST/CHEMIST THAT
COMPLETEDNERIFIED THE CALIBRATION/ANALYSIS ON PAGES 107 TO 109 . 
HNF-SD-WR-DP-258, REV. 0

Analys is Report

Averages

Fri 07-18-97 12:40:22 PM page 2

非 Sample Name

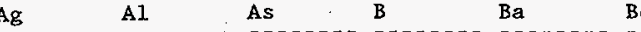

1 ICV

2 ICB

3 LLS

5 ICSAB

6 S97T001365

7. \$97T001365_D

8 ICSA

9 ICSAB

$10 \mathrm{GCV}$ Ag

$11 \mathrm{CCB}$

5.113
-.0004
.0225
.0023
.9651
.0029
.0034
.0047
.9674
5.143
-.0000

5.046

5.327

5.166

5.193

-.0001

.1032

1031

245.7

245.2

4.126

4.148

244.6

245.6

5. 107

Bi

.0061

.0100

.0360

$-.0633$

$-.0738$

$-.0814$

$-.0616$

$-.0435$

5.362

$-.0059$

.0015

.1031
-.0097

$\therefore 0074$

.0001

1. 259

1. 231

.0005

$-.0087$

$-.0037$

.0003

.4820

5.242

5.284

Cd

$\mathrm{Ge}$

Co

5.264
-.0057
.1887
-.0348
-.0153
-.0535
-.0232
-.0349
$=.0458$
5.255
.0064

5.229

5.140

5.166

5.107

$.0004 \quad .0045$

$.0101 \quad .2173$

2187

249.6

253.0

.0011

.9389

$-.0025$

1533
1467

251.0

.0007

.0027

9397

254.1

5. 165

.0142

.0228

.0258

.0250

.0296

.0020

.0180

$-.0004$

.0097

Fe

If Sample Name

$\mathrm{Cu}$

Eu

$\mathrm{K}$

.00418

.0418

$-.0018$

.0005

.0018

.0000

.4719

5.122

Be

$1 \mathrm{ICV}$

2 ICB

3 LISS

4 ICSA

5 ICSAB

6 S97T001365

7 S97T001365 D

8 ICSA

9 ICSAB

$10 \mathrm{CCV}$

11 CCB

价 Sample Name

$\begin{array}{ll}1 & \text { ICV } \\ 2 & \text { ICB } \\ 3 & \text { LLS } \\ 4 & \text { ICSA } \\ 5 & \text { ICSAB } \\ 6 & \text { S97T001365 } \\ 7 & \text { S97T001365_D } \\ 8 & \text { ICSA }\end{array}$

5. 289

$-.0004$

2154

255.5

255.3

0528

0463

254.5
4.854

$-.0007$

.0937

93.27

93.64

.0946

.0913

93.23

93.84

4.882

.0021

Mo

in

5. 044

- 0001

.0190

$-.0070$

.4461

.0168

.0165

$-.0065$
5. 132

$-.0011$

.0982

$-.0122$

$-.0066$

.0038

.0030

$-0124$
5.006

$-.0654$

4253

.1438

.1224

i. 342

1. 191

.2795

.0020

5.031

$-.0090$

$\mathrm{Na}$

5.043

.0046

.1546

204. 1

200.8

31.82

32.01
La

$-.0002$

.1065

$-.0040$

$-.0024$

0034

.0031

-.0017

$-.0026$

5. 338

.0006

Nd

5.256

.0002

.2061

.0015

.0015

.0051

.0051
5. 351

.0001

.0002

.4928

-.0002

.0002

.0002

.4934

5.397

$\mathrm{Cr}$

5.192

-.0005

.0209

-.0029

4710

.0314

- 0012

.4724

5.214

.0018

LI

5. 247

.0001

.0212

.0024

i. 023

.1160

.1153

.0025

1.025

5. 371

$-.0005$

i

5. 139

$-.0043$

.0367

$-.0084$

.9336

.0080

.0030 
HNF-SD-WM-DP-258, REV. 0

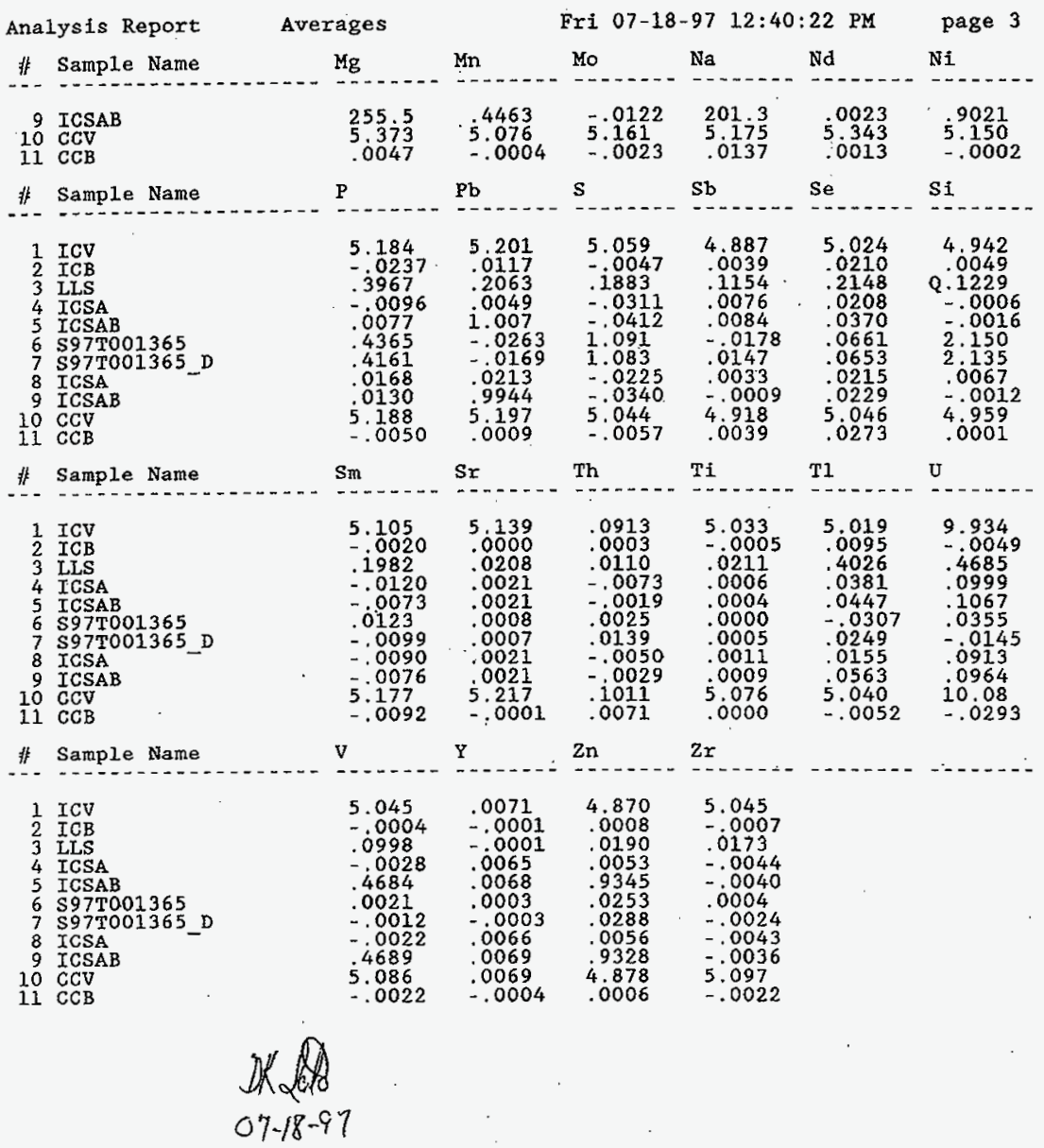




\section{LABCORE Data Entry Template for Worklist\# 19462}

Analyst: $\quad D K \mathbb{F}_{\varepsilon} \psi_{0}$ Instrument: ICP01 Book\# $\underline{47 B 48 B}$

Method: LA-505-151/16+Rev/Mod E-1

Worklist Comment: ICP BY-101 (FUSION)

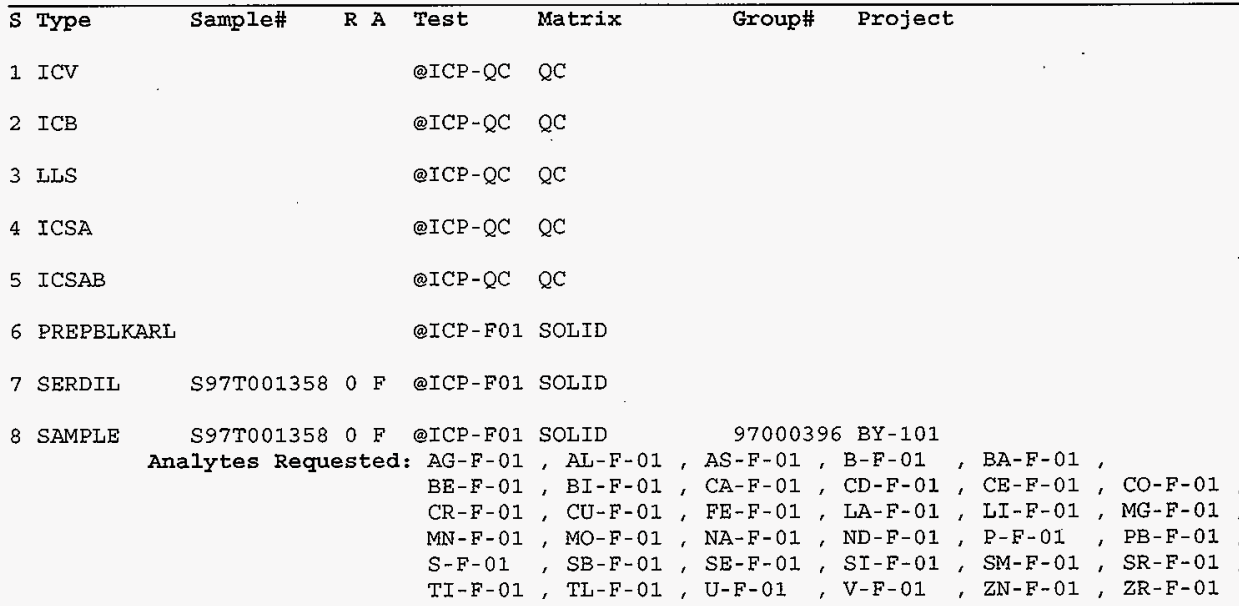

9 DUP S97T001358 O F @ICP-F01 SOLID

10 SPK S97T001358 O F @ICP-F01 SOLID

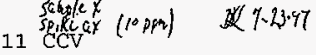

$12 \mathrm{CCB}$

$@ I C P-Q C \quad Q C$

$@ I C P-Q C \quad Q C$

13 SERDIL

S97T001363 O F @ICP-F01 SOLID

14 SAMPLE

S97T001363 OF@ICP-F01 SOLID

$97000397 B Y-101$

Analytes Requested: $A G-F-01, A L-F-01, A S-F-01, B-F-01, B A-F-01$,

BE-F-01，BI-F-01，CA-F-01，CD-F-01，CE-F-01，CO-F-01，

CR-F-01， CU-F-01，FE-F-01，LA-F-01，LI-F-01， MG-F-01，

MN-F-01，MO-F-01，NA-F-01，ND-F-01，P-F-01，PB-F-01，

Data Entry Comments:

$S=$ Worklist Slot Number, $R=$ Replicate Number, $A=$ Aliquot Code. 


\section{LABCORE Data Entry Template for Worklist\# 19462}

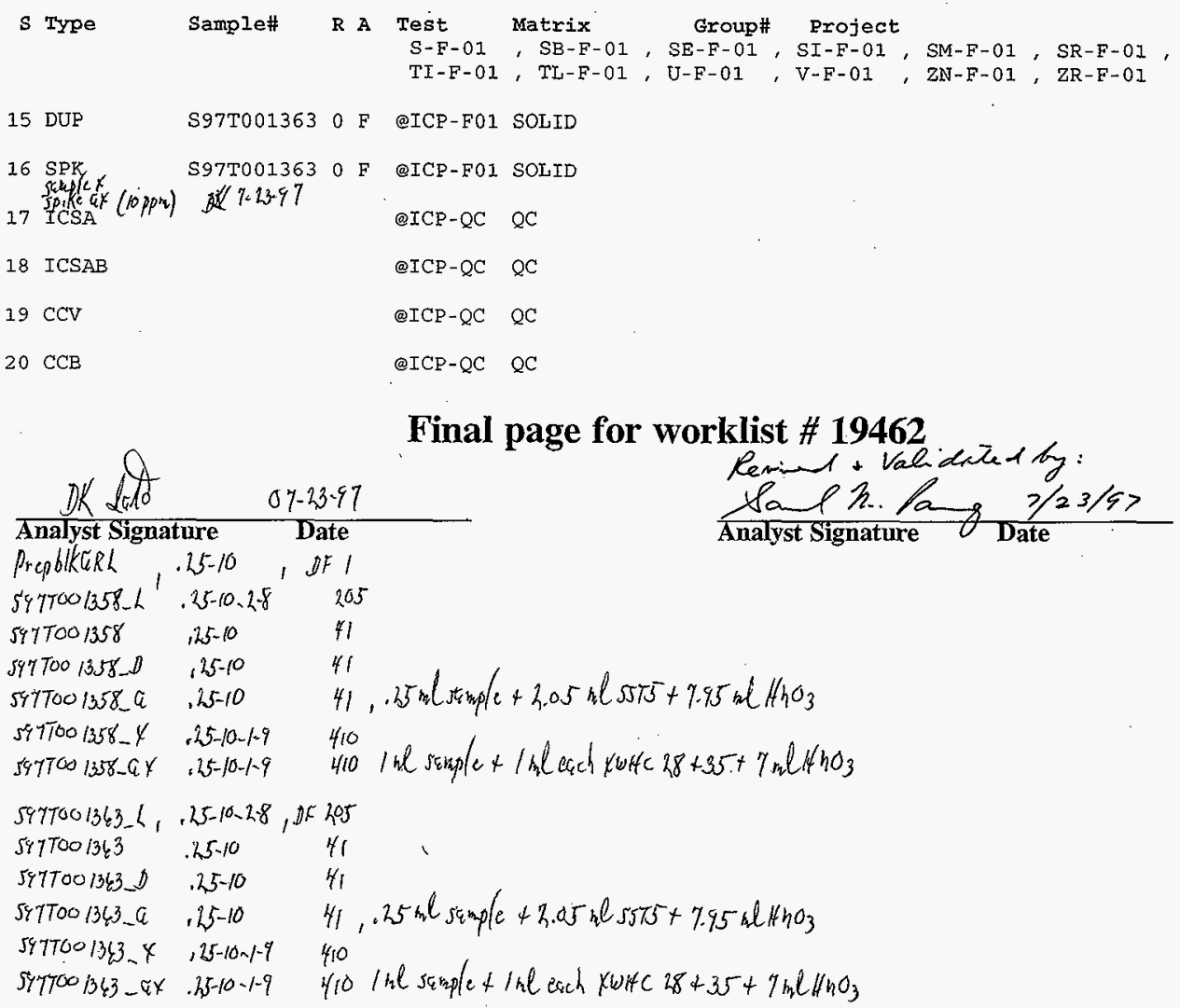

Data Entry Comments:

$S=$ Worklist Slot Number, $R=$ Replicate Number, $A=$ Aliquot Code. 
Identity $1:$ ICV Identity 2: Quality control Task name : OPTIMA Sample Weight : On-Peak Integrations : $\begin{array}{lll}1.0000 & \text { Solution Volume : } & 1.00 \\ : 3 \text { off-Peak Integrations : } 1\end{array}$

30:21 AM July 23, 1997

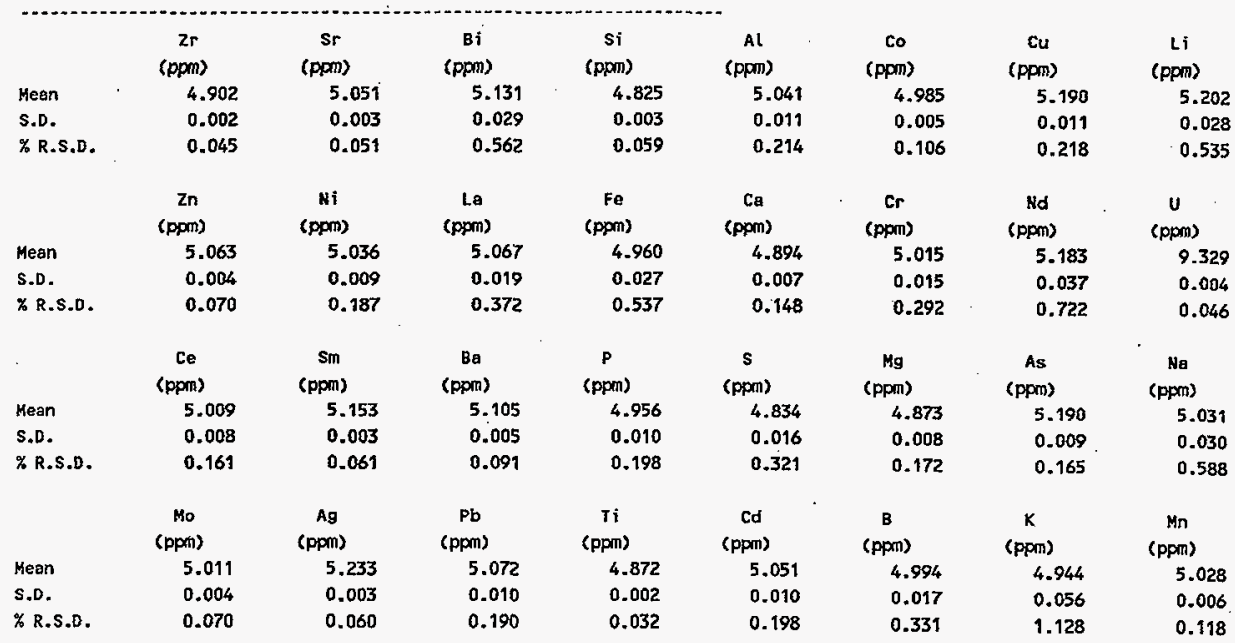

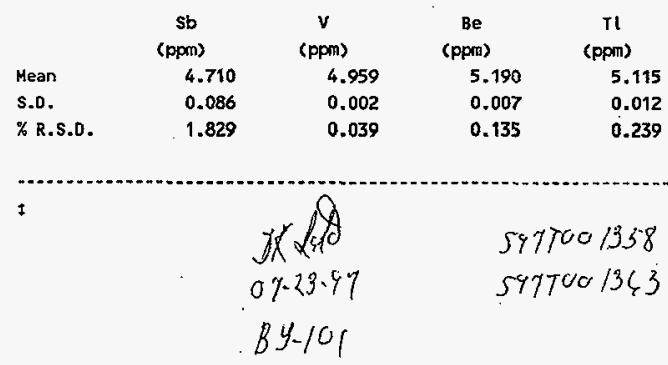

Post Lph: SIGNATURE AgOVE REPRESENTS CHEMICAL TECHNOLOGISTICHEMIST THAT COMPLETEDVERIFIED THE CALIBRATIONAANALYSIS ON PAGES 112 TO/35.

$597700 / 358$

$$
\mu_{a}=\frac{\left(\frac{4495.201}{410}\right)-\left(\frac{463.8}{410}\right)}{10} \times 100=98.4 \%
$$

5977001363

$$
M_{a}=\underbrace{\left(\frac{4330.879}{410}\right)-\left(\frac{553.534}{410}\right)}_{10} \times 100=92.1 \%
$$


Identity 1: ICB Identity 2: quality Control 10:25 AM July 23, 1997 rask name : OPIIMA Sample Weight : $\quad 1.0000$ solution Voiume : $\quad 1.00$ on-Peak integrations : 3 off-Peak Integrations : 1

\begin{tabular}{|c|c|c|c|c|c|c|c|c|}
\hline & $\begin{array}{c}2 r \\
\text { (ppm) }\end{array}$ & $\begin{array}{c}s r \\
\text { (ppm) }\end{array}$ & $\begin{array}{c}B i \\
(p p m)\end{array}$ & $\begin{array}{c}\text { sì } \\
\text { (ppm) }\end{array}$ & $\begin{array}{c}\text { Al } \\
\text { (ppm) }\end{array}$ & $\underset{\text { (ppm) }}{\text { Co }}$ & $\underset{(p p m)}{c u}$ & $\underset{\text { (ppm) }}{\text { Li }}$ \\
\hline Mean & 0.000 & -0.001 & -0.014 & 0.002 & -0.021 & 0.000 & -0.001 & -0.001 \\
\hline S.D. & 0.0 .02 & 0.000 & 0.010 & 0.005 & 0.014 & 0.002 & 0.001 & 0.000 \\
\hline \multirow[t]{2}{*}{ \& R.S.D. } & 488.003 & 27.152 & 67.265 & 332.871 & 65.173 & 21646.796 & 82.879 & 32.922 \\
\hline & $\begin{array}{c}2 n \\
(\mathrm{ppm})\end{array}$ & $\underset{\text { (ppon) }}{\mathbf{N i}}$ & $\begin{array}{c}\text { La } \\
\text { (ppm) }\end{array}$ & $\begin{array}{c}\text { Fe } \\
(p p m)\end{array}$ & $\begin{array}{c}\mathrm{Ca} \\
(\mathrm{ppm})\end{array}$ & $\underset{\text { (ppom) }}{\mathbf{C r}}$ & $\begin{array}{c}\text { Nd } \\
\text { (ppm) }\end{array}$ & $\underset{\text { (ppm) }}{u}$ \\
\hline Mean & -0.000 & 0.004 & 0.003 & -0.004 & 0.001 & -0.003 & -0.029 & 0.038 \\
\hline S.D. & 0.000 & 0.001 & 0.001 & 0.004 & 0.000 & 0.002 & 0.002 & 0.066 \\
\hline \multirow[t]{2}{*}{ \% R.S.D. } & 5.699 & 37.774 & 29.661 & 100.085 & 5.774 & 75.369 & 7.351 & 174.935 \\
\hline & $\begin{array}{c}\text { Ce } \\
\text { (ppm) }\end{array}$ & $\begin{array}{c}\text { Sm } \\
\text { (ppm) }\end{array}$ & $\begin{array}{c}\text { Ba } \\
\text { (ppm) }\end{array}$ & $\underset{(\mathrm{ppm})}{P}$ & $\stackrel{s}{(\mathrm{ppm})}$ & $\begin{array}{c}\mathrm{Mg} \\
(\mathrm{ppm})\end{array}$ & $\begin{array}{c}\text { As } \\
\text { (Ppm) }\end{array}$ & $\begin{array}{c}\mathrm{Ne} \\
\text { (ppm) }\end{array}$ \\
\hline Mean & -0.052 & 0.022 & -0.001 & 0.008 & -0.004 & 0.001 & -0.003 & -0.014 \\
\hline S.D. & 0.017 & 0.017 & 0.000 & 0.004 & 0.003 & 0.000 & 0.002 & 0.008 \\
\hline \multirow[t]{2}{*}{ \% R.S.D. } & 32.634 & 73.679 & 38.442 & 50.227 & 72.768 & 29.995 & 74.268 & 58.536 \\
\hline & $\begin{array}{c}\text { Mo } \\
\text { (ppm) }\end{array}$ & $\begin{array}{c}\mathbf{A g} \\
(\mathrm{ppm})\end{array}$ & $\begin{array}{c}\mathrm{Pb} \\
\text { (pprn) }\end{array}$ & $\begin{array}{c}\mathrm{Ti} \\
\text { (ppm) }\end{array}$ & $\begin{array}{c}\text { cd } \\
\text { (ppm) }\end{array}$ & $\begin{array}{c}\text { B } \\
\text { (pponi) }\end{array}$ & $\underset{(\mathrm{ppm})}{K}$ & $\begin{array}{c}\mathrm{Mn} \\
(p, p m)\end{array}$ \\
\hline Kean & -0.001 & -0.004 & 0.007 & -0.001 & 0.001 & -0.004 & -0.185 & 0.000 \\
\hline S.D. & 0.001 & 0.002 & 0.008 & 0.001 & 0.001 & 0.001 & 0.031 & 0.000 \\
\hline \% R.S.D. & 150.111 & 44.164 & 105.822 & 160.613 & 294.982 & 13.524 & 16.859 & 176.432 \\
\hline
\end{tabular}

\begin{tabular}{lrrrr} 
& \multicolumn{1}{c}{$\mathrm{Sb}$} & $\mathrm{V}$ & \multicolumn{1}{c}{$\mathrm{Be}$} & \multicolumn{1}{c}{$\mathrm{Tl}$} \\
& (ppm) & \multicolumn{1}{c}{ (ppm) } & \multicolumn{1}{c}{ (ppm) } & \multicolumn{1}{c}{ (ppm) } \\
Mean & 0.024 & -0.006 & -0.001 & -0.099 \\
S.D. & 0.015 & 0.001 & 0.000 & 0.042 \\
KR.S.0. & 61.447 & 18.653 & 14.755 & 42.286
\end{tabular}


HNF-SD-WM-DP-258, REV. 0

Identity 1: L.S Identity 2: Quality Control $\quad$ 10:27 AM July 23, 1997

Task name : OPTIMA

Sample Weight : $\quad 1.0000$ solution volume : 1.00

on-Pesk integrations: 3 off-Peak Integrations : 1

\begin{tabular}{|c|c|c|c|c|c|c|c|c|}
\hline & $\begin{array}{c}\mathbf{Z r} \\
\text { (Ppm) }\end{array}$ & $\begin{array}{c}\mathrm{sr} \\
(\mathrm{ppm})\end{array}$ & $\begin{array}{c}\mathbf{B i} \\
(\mathrm{ppm})\end{array}$ & $\begin{array}{c}\mathrm{si} \\
(\mathrm{ppm})\end{array}$ & $\begin{array}{c}\text { AL } \\
\text { (ppm) }\end{array}$ & $\begin{array}{c}\text { co } \\
\text { (ppm) }\end{array}$ & $\underset{(\mathrm{ppm})}{\mathrm{Cu}}$ & $\underset{\text { (ppon) }}{L i}$ \\
\hline Hesn & 0.022 & 0.021 & 0.179 & 0.115 & 0.103 & 0.041 & 0.022 & 0.022 \\
\hline S.D. & 0.003 & 0.000 & 0.007 & 0.004 & 0.008 & 0.003 & 0.001 & 0.000 \\
\hline \multirow[t]{2}{*}{ \% R.S.O. } & 11.269 & 1.792 & 3.993 & 3.304 & 7.860 & 8.561 & 3.087 & 2.182 \\
\hline & $\begin{array}{c}Z n \\
(p p m)\end{array}$ & $\begin{array}{c}\boldsymbol{\mu j} \\
\text { (ppm) }\end{array}$ & $\begin{array}{c}\text { Lo } \\
\text { (ppori) }\end{array}$ & $\begin{array}{c}\mathrm{Fe} \\
(\mathrm{ppm})\end{array}$ & $\begin{array}{c}\mathrm{Ca} \\
(\mathrm{ppm})\end{array}$ & $\underset{(p p m)}{C r}$ & $\begin{array}{c}\text { Nd } \\
\text { (pprm) }\end{array}$ & $\underset{(p p m)}{U}$ \\
\hline Mean & 0.021 & 0.043 & 0.103 & 0.097 & 0.201 & 0.018 & 0.189 & 0.451 \\
\hline s.o. & 0.000 & 0.000 & 0.002 & 0.004 & 0.001 & 0.003 & 0.011 & 0.055 \\
\hline \multirow[t]{2}{*}{ \% R.S.O. } & 2.129 & 0.482 & 2.046 & 3.695 & 0.581 & 14.601 & 5.789 & 12.294 \\
\hline & $\begin{array}{c}\text { Ce } \\
\langle p p m\rangle)\end{array}$ & $\underset{\langle p p m\rangle}{S m}$ & $\stackrel{\mathrm{Ba}}{(p p m)}$ & $\underset{(p p m)}{P}$ & $\stackrel{s}{\text { (ppoms) }}$ & $\begin{array}{c}\mathrm{Mg} \\
(\mathrm{ppm})\end{array}$ & $\begin{array}{c}\text { As } \\
\text { (ppm) }\end{array}$ & $\begin{array}{c}\mathrm{Ma} \\
\text { (ppm) }\end{array}$ \\
\hline Mean & 0.180 & 0.243 & 0.105 & 0.422 & 0.210 & 0.198 & 0.219 & 0.205 \\
\hline S.D. & 0.016 & 0.012 & 0.001 & 0.005 & 0.003 & 0.001 & 0.003 & 0.004 \\
\hline \multirow[t]{2}{*}{ \% R.S.D. } & 8.665 & 5.135 & 0.636 & 1.224 & 1.502 & 0.728 & 1.354 & 1.910 \\
\hline & $\begin{array}{c}\text { Mo } \\
\text { (ppm) }\end{array}$ & $\begin{array}{c}\text { Ag } \\
\text { (ppm) }\end{array}$ & $\begin{array}{c}\mathrm{Pb} \\
\text { (ppm) }\end{array}$ & $\begin{array}{c}T i \\
(p p m)\end{array}$ & $\underset{\text { (ppm) }}{c d}$ & $\begin{array}{c}\mathrm{B} \\
\text { (ppm) }\end{array}$ & $\underset{(\mathrm{ppm})}{K}$ & $\underset{(\mathrm{ppm})}{\mathrm{Mn}}$ \\
\hline Mean & 0.100 & 0.022 & 0.227 & 0.020 & 0.014 & 0.097 & 0.267 & 0.021 \\
\hline S.o. & 0.001 & 0.001 & 0.004 & 0.001 & 0.001 & 0.003 & 0.017 & 0.001 \\
\hline \multirow[t]{2}{*}{ \% R.S.D. } & 0.894 & 5.718 & 1.683 & 3.765 & 3.742 & 3.231 & 6.471 & 2.523 \\
\hline & $\begin{array}{c}\text { Sb } \\
\text { (ppm) }\end{array}$ & $\begin{array}{c}V \\
(p p x i)\end{array}$ & $\begin{array}{c}\mathrm{Be} \\
\text { (pprn) }\end{array}$ & $\begin{array}{c}\text { Tl } \\
\text { (ppon) }\end{array}$ & & & & \\
\hline Mean & 0.085 & 0.099 & 0.010 & 0.319 & & & & \\
\hline S.D. & 0.031 & 0.001 & 0.001 & 0.037 & & & & \\
\hline$\%$ R.S.O. & 36.126 & 1.306 & 5.926 & 11.749 & & & & \\
\hline
\end{tabular}




\section{HNF-SD-WR-DP-258, REV. 0}

Identity $3:$ JCSA Task name : OPTIMA

Sample Height :

on-Peak Integrations : 3
Identity 2: Quality contral

$10: 31$ AM July 23, 1997

\begin{tabular}{|c|c|c|c|c|c|c|c|c|}
\hline & $z r$ & $\mathrm{sr}$ & Bi & si & Al & Co & cu & Lt \\
\hline & (ppon) & (ppm) & (ppon) & (ppm) & (ppm) & 〈ppm & (ppan) & $(p p m)$ \\
\hline Mean & 0.001 & 0.002 & -0.037 & 0.012 & 201.341 & 0.005 & 0.000 & 0.001 \\
\hline S.D. & 0.001 & 0.000 & 0.007 & 0.002 & 0.625 & 0.002 & 0.000 & 0.000 \\
\hline \multirow[t]{2}{*}{ K R.S.D. } & 100.416 & 1.786 & 19.333 & 17.342 & 0.311 & 36.845 & 34.188 & 17.555 \\
\hline & $\begin{array}{c}\mathrm{Zn} \\
\text { (PPOA) }\end{array}$ & $\underset{\text { (pponi) }}{N i}$ & $\underset{\text { (ppon) }}{\mathrm{La}}$ & $\begin{array}{c}\text { Fe } \\
\text { (ppm) }\end{array}$ & $\begin{array}{c}\mathrm{Ca} \\
\text { (pam) }\end{array}$ & $\begin{array}{c}\mathrm{Cr} \\
\text { (Ppm) }\end{array}$ & $\begin{array}{c}\text { Nd } \\
\text { (ppm) }\end{array}$ & $\underset{(p p m)}{U}$ \\
\hline Mean & 0.006 & 0.002 & -0.004 & 93.863 & 102.479 & -0.002 & 0.039 & -0.033 \\
\hline S.D. & 0.000 & 0.001 & 0.003 & 0.047 & 0.233 & 0.001 & 0.008 & 0.006 \\
\hline \multirow[t]{2}{*}{ \% R.S.D. } & 4.388 & 51.897 & 85.637 & 0.051 & 0.227 & 50.468 & 21.241 & 19.772 \\
\hline & $\begin{array}{c}\text { Ce } \\
\text { (ppm) }\end{array}$ & $\begin{array}{c}\mathrm{Sn} \\
(\mathrm{ppm})\end{array}$ & $\begin{array}{c}\mathrm{Ba} \\
\text { (ppm) }\end{array}$ & $\begin{array}{c}P \\
(\mathrm{ppm})\end{array}$ & $\begin{array}{c}s \\
\text { (ppm) }\end{array}$ & $\begin{array}{c}M g \\
(p p m)\end{array}$ & $\begin{array}{c}\text { As } \\
\text { (ppm) }\end{array}$ & $\begin{array}{c}\text { Ha } \\
\text { (ppm) }\end{array}$ \\
\hline Mean & 0.024 & 0.053 & 0.002 & 0.005 & -0.111 & 100.934 & -0.049 & 194.021 \\
\hline S.D. & 0.004 & 0.006 & 0.000 & 0.004 & 0.005 & 0.178 & 0.008 & 0.683 \\
\hline \multirow[t]{3}{*}{ \% R.S.D. } & 17.232 & 11.208 & 0.990 & 65.458 & 4.676 & 0.177 & 16.519 & 0.352 \\
\hline & Mo & Ag & $P b$ & $\mathbf{T i}$ & $c d$ & $\mathbf{B}$ & $\kappa$ & $\mathrm{Hn}$ \\
\hline & (ppm) & (ppm) & (ppm) & (ppm) & (ppm) & (ppm) & (ppn) & (ppm) \\
\hline Mesn & 0.000 & 0.004 & 0.017 & -0.001 & 0.003 & 0.006 & -0.333 & 0.005 \\
\hline S.D. & 0.002 & 0.000 & 0.010 & 0.000 & 0.000 & 0.001 & 0.011 & 0.001 \\
\hline \multirow[t]{2}{*}{ \% R.S.D. } & 863.049 & 3.021 & 58.885 & 14.204 & 10.714 & 20.612 & 3.445 & 14.621 \\
\hline & $\begin{array}{c}\text { sb } \\
\text { (ppm) }\end{array}$ & $\begin{array}{c}v \\
\text { (ppm) }\end{array}$ & $\begin{array}{c}\mathrm{Be} \\
\text { (ppon) }\end{array}$ & $\begin{array}{c}T l \\
\text { (ppon) }\end{array}$ & & & & \\
\hline Mean & 0.030 & -0.002 & 0.002 & -0.042 & & & & \\
\hline S.D. & 0.010 & 0.001 & 0.000 & 0.006 & & & & \\
\hline X R.S.D. & 31.814 & 36.749 & 13.133 & 14.537 & & & & \\
\hline
\end{tabular}


HNF-SD-WM-DP-258, REV. 0

Identity 1: ICSAB Identity 2: Qual ity control Task name : OPTIMA

Sample Weight : 1.0000 solution volume : on-Peak Integrations : 3 off-Peak integrations :
10:33 AM July 23, 1997

\begin{tabular}{|c|c|c|c|c|c|c|c|c|}
\hline & $\begin{array}{c}\mathrm{Zr} \\
(\mathrm{pppm})\end{array}$ & $\begin{array}{c}\text { Sr } \\
(p p m)\end{array}$ & $\begin{array}{c}\text { Bi } \\
\text { (ppm) }\end{array}$ & $\begin{array}{c}\text { si } \\
\text { (ppm) }\end{array}$ & $\begin{array}{c}\text { At } \\
\text { (ppm) }\end{array}$ & $\begin{array}{c}\text { co } \\
\text { (ppm) }\end{array}$ & $\begin{array}{c}c u \\
\text { (ppm) }\end{array}$ & $\underset{\text { (ppra) }}{L i}$ \\
\hline Mean & -0.005 & 0.002 & -0.043 & 0.001 & 198.859 & $0.4 \pi 5$ & 0.500 & 0.968 \\
\hline s.o. & 0.001 & 0.000 & 0.013 & 0.007 & 1.073 & 0.006 & 0.002 & 0.007 \\
\hline \multirow[t]{2}{*}{ \% R.S.D. } & 31.142 & 10.604 & 30.472 & 782.841 & 0.539 & 1.324 & 0.483 & 0.680 \\
\hline & $\underset{(\mathrm{ppm})}{\mathrm{Zn}}$ & $\underset{\text { (ppm) }}{\mathrm{Ni}}$ & $\begin{array}{c}\text { La } \\
\text { (ppon) }\end{array}$ & $\begin{array}{c}\text { Fe } \\
\text { (ppm) }\end{array}$ & $\begin{array}{c}\mathrm{Ca} \\
\text { (ppri) }\end{array}$ & $\underset{(\mathrm{pPm})}{\mathrm{Cr}}$ & $\begin{array}{c}\text { Nd } \\
\text { (ppm) }\end{array}$ & $\underset{\text { (pprn) }}{U}$ \\
\hline Mean & 0.953 & 0.960 & -0.003 & 94.540 & 101.848 & 0.481 & 0.032 & -0.109 \\
\hline s.o. & 0.013 & 0.020 & 0.002 & 1.240 & 1.204 & 0.014 & 0.011 & 0.077 \\
\hline \multirow[t]{2}{*}{ X R.S.D. } & 1.317 & 2.064 & 62.526 & 1.312 & 1.182 & 2.974 & 33.497 & 70.657 \\
\hline & $\begin{array}{c}\text { Ce } \\
\text { (ppm) }\end{array}$ & $\begin{array}{c}\mathrm{Sm} \\
\text { (ppm) }\end{array}$ & $\begin{array}{c}\text { Ba } \\
\text { (ppril) }\end{array}$ & $\begin{array}{c}P \\
\text { (ppm) }\end{array}$ & $\underset{\text { (ppan) }}{S}$ & $\underset{\text { (ppm) }}{\mathrm{Mg}}$ & $\begin{array}{c}\text { As } \\
\text { (ppm) }\end{array}$ & $\begin{array}{c}\mathrm{Ne} \\
\text { (ppom) }\end{array}$ \\
\hline Mean & 0.020 & 0.017 & 0.506 & 0.006 & -0.110 & 99.940 & -0.037 & 190.365 \\
\hline S.D. & 0.015 & 0.012 & 0.004 & 0.011 & 0.007 & 1.704 & 0.007 & 0.968 \\
\hline \multirow[t]{2}{*}{ \% R.S.D. } & 76.604 & 69.815 & 0.866 & 174.241 & 6.743 & 1.706 & 17.766 & 0.509 \\
\hline & $\begin{array}{c}\text { Mo } \\
\text { (Ppm) }\end{array}$ & $\begin{array}{c}\mathrm{Ag} \\
\text { (ppm) }\end{array}$ & $\begin{array}{c}\mathrm{Pb} \\
(p \mathrm{pm})\end{array}$ & $\begin{array}{c}T i \\
\text { (ppris) }\end{array}$ & $\begin{array}{c}\text { cd } \\
\text { (ppm) }\end{array}$ & $\stackrel{\text { B }}{\text { (pprn) }}$ & $\begin{array}{c}K \\
\text { (ppm) }\end{array}$ & $\begin{array}{c}M n \\
\text { (ppm) }\end{array}$ \\
\hline Mean & -0.002 & 1.024 & 0.994 & -0.001 & 0.958 & 0.006 & -0.250 & 0.486 \\
\hline s.o. & 0.003 & 0.011 & 0.010 & 0.001 & 0.013 & 0.002 & 0.026 & 0.009 \\
\hline \multirow[t]{2}{*}{ \% R.S.D. } & 130.165 & 1.038 & 1.033 & 51.127 & 1.376 & 25.260 & 10.294 & 1.755 \\
\hline & $\begin{array}{c}\text { Sb } \\
(p p m)\end{array}$ & $\begin{array}{c}v \\
(p p m)\end{array}$ & $\begin{array}{c}\mathrm{Be} \\
\text { (ppm) }\end{array}$ & $\begin{array}{c}\mathrm{Tl} \\
\text { (ppm) }\end{array}$ & & & & \\
\hline Mean & 0.052 & 0.480 & 0.492 & -0.065 & & & & \\
\hline S.D. & $0.035^{\circ}$ & 0.008 & 0.006 & 0.033 & & & & \\
\hline \% R.S.D. & 67.241 & 1.624 & 1.244 & 50.667 & & & & \\
\hline
\end{tabular}


Task name : OPTIMA

Sample Weight : $\quad 1.0000$ solution Volume: $\quad 1.00$

on-Peak Integrations : 3 off-Peak Integrations : 9

\begin{tabular}{|c|c|c|c|c|c|c|c|c|}
\hline & $\begin{array}{c}\mathbf{Z r} \\
\text { (ppm) }\end{array}$ & $\begin{array}{c}\mathrm{sr} \\
\text { (ppm) }\end{array}$ & $\begin{array}{c}B 1 \\
\text { (pponi) }\end{array}$ & $\begin{array}{c}\text { si } \\
\text { (ppm) }\end{array}$ & $\begin{array}{c}\text { AI } \\
\text { (ppm) }\end{array}$ & $\begin{array}{c}\text { co } \\
\text { (ppm) }\end{array}$ & $\begin{array}{c}\text { Cu } \\
\text { (pprin) }\end{array}$ & $\stackrel{\text { Li }}{\text { (ppmi) }}$ \\
\hline Hean & 0.000 & -0.000 & -0.004 & 0.018 & 0.013 & -0.001 & 0.001 & -0.000 \\
\hline S.D. & 0.001 & 0.000 & 0.006 & 0.003 & 0.008 & 0.000 & 0.001 & 0.000 \\
\hline \multirow[t]{2}{*}{ \% R.S.D. } & 483.730 & 69.282 & 138.177 & 16.350 & 65.916 & 0.888 & 80.514 & 360.429 \\
\hline & $\underset{\text { (pprin) }}{\mathrm{Zn}}$ & $\begin{array}{c}\mathrm{Ni} \\
\text { (PPn) }\end{array}$ & $\begin{array}{c}\text { La } \\
\text { (pprn) }\end{array}$ & $\begin{array}{c}\text { Fe } \\
\text { (ppris) }\end{array}$ & $\begin{array}{c}\mathrm{Ca} \\
\text { (ppm) }\end{array}$ & $\begin{array}{c}\mathrm{Cr} \\
(\mathrm{ppm})\end{array}$ & $\begin{array}{c}\text { Nd } \\
\text { (ppm) }\end{array}$ & $\underset{\text { (ppm) }}{U}$ \\
\hline Mean & 0.001 & 0.156 & 0.003 & -0.001 & 0.001 & 0.002 & -0.017 & -0.022 \\
\hline S.D. & 0.000 & 0.003 & 0.001 & 0.003 & 0.000 & 0.000 & 0.006 & 0.055 \\
\hline \multirow[t]{2}{*}{ \% R.S.D. } & 11.402 & 1.789 & 29.847 & 302.223 & 19.924 & 21.110 & 35.623 & 248.651 \\
\hline & $\begin{array}{c}\mathrm{Ce} \\
\text { (PpDA) }\end{array}$ & $\begin{array}{c}\mathrm{Sm} \\
(\mathrm{ppm})\end{array}$ & $\begin{array}{c}\mathrm{Ba} \\
\text { (ppm) }\end{array}$ & $\underset{(p p m)}{p}$ & $\begin{array}{c}s \\
\text { (ppm) }\end{array}$ & $\begin{array}{c}\mathrm{Mg} \\
\text { (ppm) }\end{array}$ & $\begin{array}{c}\text { As } \\
\text { (ppm) }\end{array}$ & $\underset{(\mathrm{ppm})}{\mathrm{Na}}$ \\
\hline Mean & -0.007 & 0.057 & 0.000 & 0.021 & -0.001 & 0.002 & -0.005 & 0.087 \\
\hline s.o. & 0.013 & 0.010 & 0.000 & 0.002 & 0.001 & 0.000 & 0.002 & 0.003 \\
\hline \multirow[t]{2}{*}{ * R.S.D. } & 193.225 & 16,968 & 321.667 & 11.190 & 150.459 & 4.225 & 40.142 & 3.985 \\
\hline & $\begin{array}{c}\text { Ho } \\
\text { (Ppm) }\end{array}$ & $\begin{array}{c}\text { Ag } \\
\text { (pprin) }\end{array}$ & (ppom) & $\begin{array}{c}T i \\
\text { (ppmin) }\end{array}$ & $\begin{array}{c}\text { Cd } \\
\text { (ppm) }\end{array}$ & (ppm) & $\underset{(\mathrm{ppm})}{\mathrm{K}}$ & $\begin{array}{c}\mathrm{Mn} \\
\text { (ppm) }\end{array}$ \\
\hline Mean & 0.000 & 0.000 & 0.028 & -0.001 & 0.002 & -0.007 & 270.351 & 0.001 \\
\hline S.D. & 0.002 & 0.001 & 0.007 & 0.000 & 0.001 & 0.001 & 1.779 & 0.000 \\
\hline \% R.S.D. & 708.634 & 236.191 & 25.612 & 34.982 & 26.010 & 9.866 & 0.658 & 0.693 \\
\hline
\end{tabular}

\begin{tabular}{|c|c|c|c|c|}
\hline & $\begin{array}{c}\text { Sb } \\
\text { (ppm) }\end{array}$ & $\begin{array}{c}v \\
\text { (ppm) }\end{array}$ & $\begin{array}{c}\text { Be } \\
\text { (ppm) }\end{array}$ & $\underset{\text { (pprn) }}{\mathrm{rl}}$ \\
\hline Mean & 0.033 & -0.001 & -0.000 & -0.059 \\
\hline S.D. & 0.022 & 0.001 & 0.000 & 0.030 \\
\hline \% R.S.D. & 66.738 & 49.431 & 174.470 & 50.938 \\
\hline
\end{tabular}


identity 1: 595T001358_L Identity 2: Fusion,..25-10-2-8mt Task name : OPT IMA

Sample Weight : 1.0000 solution Volume: 205.00

on-Peak Integrations : 3 off-Peak Integrations : 1

\begin{tabular}{|c|c|c|c|c|c|c|c|c|}
\hline & $\begin{array}{c}\mathrm{Zr} \\
\text { (ppn) }\end{array}$ & $\begin{array}{c}\mathrm{sr} \\
\text { (ppons) }\end{array}$ & $\underset{(p p m)}{B i}$ & $\begin{array}{c}\text { si } \\
\text { (ppm) }\end{array}$ & $\underset{\text { (ppm) }}{\text { Al }}$ & $\begin{array}{c}\text { Co } \\
(\mathrm{ppm})\end{array}$ & $\begin{array}{c}C u \\
\text { (PPX) }\end{array}$ & $\underset{\text { (ppom) }}{L \bar{i}}$ \\
\hline Mesn & -0.249 & -0.046 & -4.122 & 15.358 & 65.923 & 0.121 & -0.046 & 0.687 \\
\hline S.D. & 0.142 & 0.014 & 0.800 & 0.495 & 0.333 & 0.411 & 0.018 & 0.009 \\
\hline \multirow[t]{2}{*}{ \% R.S.D. } & 57.164 & 30.000 & 19.410 & 3.221 & 0.505 & 338.831 & 38.609 & 1.303 \\
\hline & $\begin{array}{c}\mathrm{Zn} \\
\text { (pprt) }\end{array}$ & $\begin{array}{c}\text { Ni } \\
\text { (ppon) }\end{array}$ & $\begin{array}{c}\text { Lo } \\
\text { (ppm) }\end{array}$ & $\begin{array}{c}\mathrm{Fe} \\
\text { (ppm) }\end{array}$ & $\begin{array}{c}\mathrm{Ca} \\
\text { (ppon) }\end{array}$ & $\begin{array}{c}\mathbf{C r} \\
\text { (ppm) }\end{array}$ & $\begin{array}{c}\text { Nd } \\
\text { (pponi) }\end{array}$ & $\underset{\text { (pPRn) }}{U}$ \\
\hline Mean & 0.034 & 8.357 & 0.657 & 1.012 & 1.295 & 23.940 & -4.778 & -8.735 \\
\hline S.D. & 0.039 & 1.857 & 0.166 & 0.400 & 0.015 & 0.340 & 1.399 & 6.996 \\
\hline \multirow[t]{2}{*}{ \% R.S.D. } & 114.439 & 22.217 & 25.241 & 39.511 & 1.132 & 1.422 & 29.276 & 70.928 \\
\hline & $\begin{array}{c}\text { Ce } \\
\text { (ppm) }\end{array}$ & $\begin{array}{c}\mathrm{sm} \\
\text { (ppm) }\end{array}$ & $\begin{array}{c}\text { Ba } \\
\text { (ppm) }\end{array}$ & $\underset{\text { (ppm) }}{p}$ & $\underset{\text { (ppon) }}{s}$ & $\begin{array}{c}\mathrm{Mg} \\
\text { (ppm) }\end{array}$ & $\begin{array}{c}\text { As } \\
\text { (ppm) }\end{array}$ & $\underset{\text { (ppm) }}{\mathrm{Na}}$ \\
\hline Mean & -5.121 & 10.070 & -0.054 & 46.283 & -1.230 & 1.087 & 0.325 & 486.541 \\
\hline S.D. & 2.206 & 1.590 & 0.053 & 1.485 & 0.500 & 0.037 & 0.598 & 1.876 \\
\hline \% R.S.D. & 43.086 & 15.794 & 98.133 & 3.207 & 40.626 & 3.411 & 184.026 & 0.386 \\
\hline$\cdot$ & $\begin{array}{c}\text { Mo } \\
\text { (ppom) }\end{array}$ & $\underset{\text { (ppm) }}{\text { Ag }}$ & $\begin{array}{c}P b \\
\text { (ppm) }\end{array}$ & $\begin{array}{c}T i \\
\text { (ppra) }\end{array}$ & $\underset{\text { (ppm) }}{\text { Cd }}$ & $\begin{array}{c}\text { B } \\
\text { (ppora) }\end{array}$ & $\begin{array}{c}K \\
\text { (ppom) }\end{array}$ & $\begin{array}{c}\text { Mn } \\
\text { (ppm) }\end{array}$ \\
\hline Mean & -0.377 & 0.001 & 1.598 & 0.069 & 0.390 & -1.922 & 19141.110 & 0.071 \\
\hline S.D. & 0.212 & 0.164 & 2.354 & 0.072 & 0.062 & 0.199 & 73.119 & 0.083 \\
\hline \% R.S.D. & 56.177 & 25849.527 & 147.331 & 103.863 & 15.829 & 10.353 & 0.382 & 117.134 \\
\hline
\end{tabular}

\begin{tabular}{lrrrr} 
& \multicolumn{1}{c}{ Sb } & \multicolumn{1}{c}{$v$} & \multicolumn{1}{c}{ Be } & \multicolumn{1}{c}{ Tl } \\
& (ppm) & \multicolumn{1}{c}{ (ppm) } & \multicolumn{1}{c}{ (ppm) } & \multicolumn{1}{c}{ (ppm) } \\
Hean & 3.300 & -0.429 & -0.184 & -11.849 \\
S.D. & 6.749 & 0.085 & 0.055 & 2.683 \\
\% R.S.D. & 204.521 & 19.922 & 29.844 & 22.643
\end{tabular}

10:42 AM suly 23, 1997 
Identity 1: \$95T001358 Identity 2: Fusion, 25-10 al 10:45 AM July 23, 1997

HNF-SD-WM-DP-258, REV. 0 Task name : OPTIMA

Sample Height: $\quad 1.0000$ Solution Volume : $\quad 41.00$

on-peak Integrations : 3 off-Pegk Integrations : 1

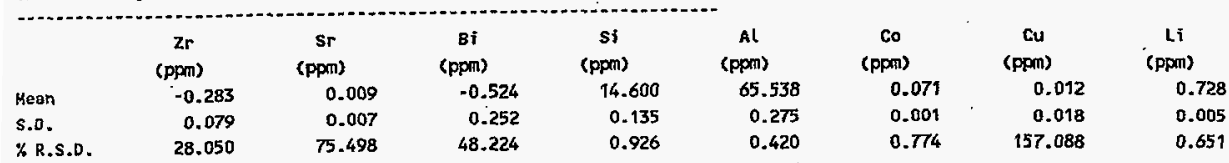

\begin{tabular}{|c|c|c|c|c|c|c|c|c|}
\hline & $\underset{\text { (ppm) }}{\mathrm{Zn}}$ & $\begin{array}{c}\mathbf{H i} \\
(\mathrm{ppm})\end{array}$ & $\begin{array}{c}\text { La } \\
\text { (pprn) }\end{array}$ & $\begin{array}{c}\mathrm{Fe} \\
\text { (PPR) }\end{array}$ & $\begin{array}{c}\text { Ca } \\
(\mathrm{ppm})\end{array}$ & $\underset{(\mathrm{ppm})}{\mathrm{Cr}}$ & $\begin{array}{c}\text { Nd } \\
\text { (ppm) }\end{array}$ & $\underset{(\mathrm{ppm})}{\mathrm{U}}$ \\
\hline Mean & 0.113 & 6.356 & -0.269 & 1.875 & 1.428 & 23.770 & -1.232 & -9.548 \\
\hline S.D. & 0.012 & 0.044 & 0.144 & 0,037 & 0.002 & 0.393 & 0.438 & 2.468 \\
\hline \multirow[t]{2}{*}{ \% R.S.O. } & 10.710 & 0.689 & 53.493 & 1.988 & 0.119 & 1.652 & 35.565 & 25.847 \\
\hline & $\begin{array}{c}\mathrm{Ce} \\
(\mathrm{ppm})\end{array}$ & $\underset{\text { (ppm) }}{5 m}$ & $\begin{array}{c}\text { Ba } \\
\text { (ppm) }\end{array}$ & $\begin{array}{c}P \\
\text { (ppm) }\end{array}$ & $\underset{\text { (pporn) }}{s}$ & $\begin{array}{c}\mathrm{Mg} \\
(\mathrm{ppm})\end{array}$ & $\begin{array}{c}\text { As } \\
\text { (ppm) }\end{array}$ & $\begin{array}{c}\mathrm{Na} \\
\text { (ppm) }\end{array}$ \\
\hline Mean & -1.324 & 0.969 & 0.015 & 44,078 & -0.469 & 0.581 & -0.108 & 479.301 \\
\hline S.D. & 0.522 & 0.472 & 0.009 & 0.512 & 0.100 & 0.000 & 0.188 & 4.147 \\
\hline \multirow[t]{2}{*}{ \% R.S.D. } & 39.432 & 48.700 & 59.766 & 1.162 & 21.407 & 0.001 & 175.000 & 0.865 \\
\hline & $\begin{array}{c}\text { Mo } \\
\text { (ppom) }\end{array}$ & $\begin{array}{c}\text { Ag } \\
\text { (ppm) }\end{array}$ & $\begin{array}{c}\mathrm{Pb} \\
(\mathrm{ppm})\end{array}$ & $\begin{array}{c}T i \\
\text { (ppm) }\end{array}$ & $\underset{\text { (ppori) }}{\text { Cd }}$ & $\begin{array}{c}\text { B } \\
\text { (ppm) }\end{array}$ & $\begin{array}{c}K \\
(p p m)\end{array}$ & $\underset{\text { (ppxn) }}{\text { Non }}$ \\
\hline Mean & -0.059 & 0.012 & 0.695 & 0.096 & 0.213 & -0.426 & 18528.243 & 0.143. \\
\hline S.D. & 0.007 & 0.046 & 0.430 & 0.031 & 0.033 & 0.077 & 112.133 & 0.016 \\
\hline *R.S.D. & 11.311 & 391.298 & 61.811 & 32.631 & 15.345 & 18.126 & 0.605 & 11.405 \\
\hline
\end{tabular}

\begin{tabular}{|c|c|c|c|c|}
\hline & $\begin{array}{c}s b \\
\text { (ppm) }\end{array}$ & $\begin{array}{c}v \\
(\mathrm{ppm})\end{array}$ & $\begin{array}{c}\mathrm{Be} \\
\text { (ppm) }\end{array}$ & $\begin{array}{c}\text { Tl } \\
\text { (ppm) }\end{array}$ \\
\hline Mesn & 0.825 & -0.022 & -0.028 & -4.942 \\
\hline s.o. & 1.107 & 0.037 & 0.000 & 1.641 \\
\hline \% R.S.D. & 134.219 & 168.542 & 1.625 & 33.206 \\
\hline
\end{tabular}


Identity 1: s95T001358_D Identity 2: Fusion, -25-10 mt Task name : OPTIMA

Sample Height :

1.0000 solution Volume :

41.00

on-Peak Integrations : 3 off-Peak Integrations : 1
10:48 AM July 23, 1997

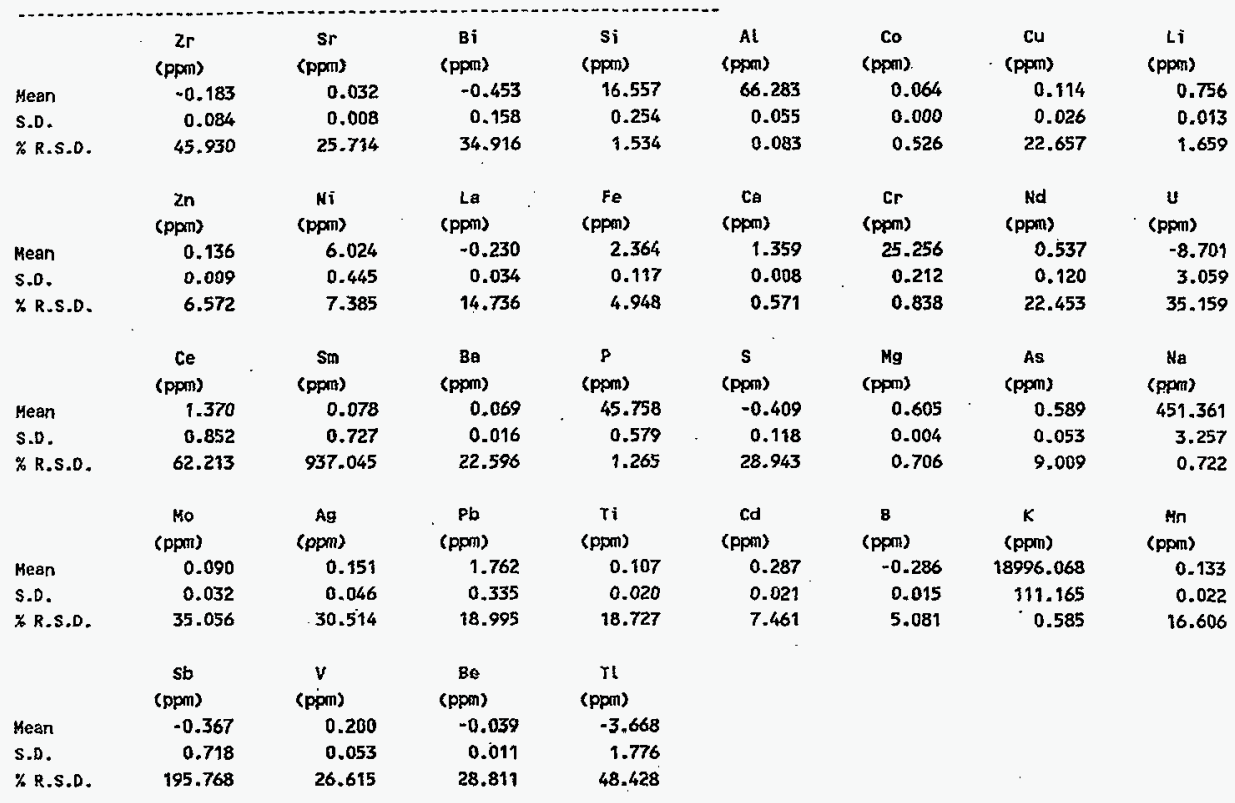


Identity 1: 595r001358_A Identity 2: Fusion, .25-10 ml cask nane : OPTIMA

Sample Height :

1.0000 solution volume : $\quad \mathbf{4 1 . 0 0}$

on-peak Integrations : 3 off-Peak Integrations : 1
10.51 AM July 23, 1997

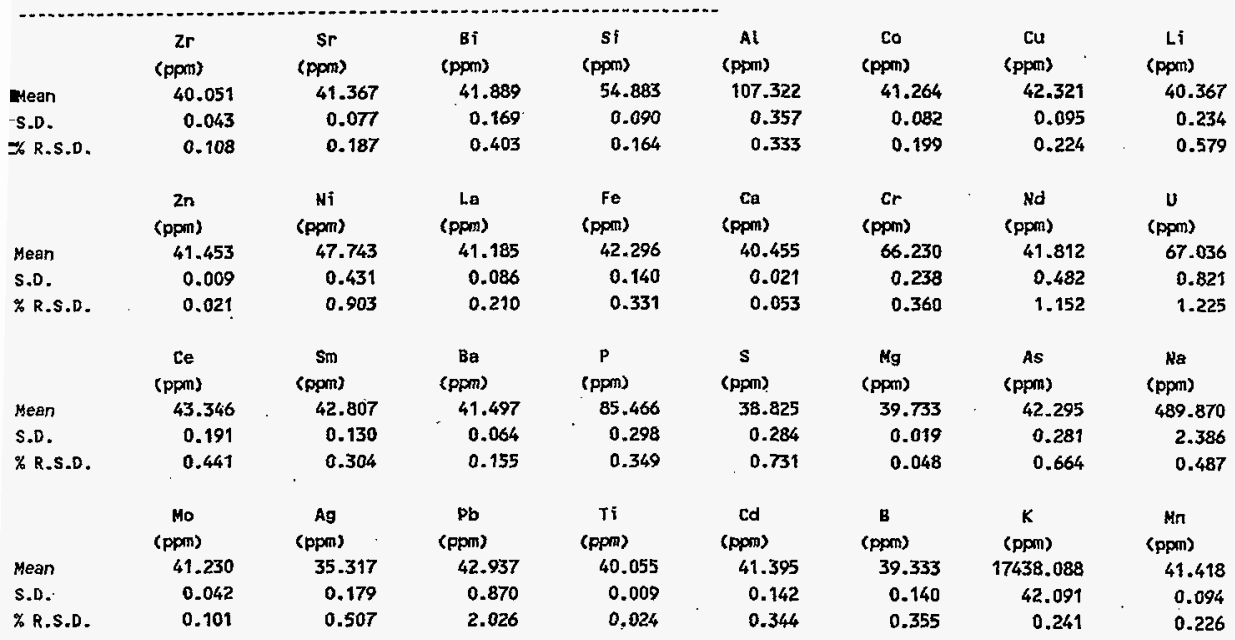

\begin{tabular}{|c|c|c|c|c|}
\hline & $\begin{array}{c}\text { sb } \\
\text { (ppm) }\end{array}$ & $\begin{array}{c}v \\
\text { (ppon) }\end{array}$ & $\begin{array}{c}\text { Be } \\
\text { (ppnn) }\end{array}$ & $\begin{array}{c}T l \\
\text { (ppm) }\end{array}$ \\
\hline ean & 39.839 & 41.103 & 40.938 & 41.031 \\
\hline 5.0. & 0.773 & 0.066 & 0.069 & 1.049 \\
\hline R.S.D. & 1.939 & 0.162 & 0.169 & 2.556 \\
\hline
\end{tabular}


Identity 1: $5957001358 \_X$ Identity 2: Fusion, .25-10-1-9 ml

10:54 AM July 23, 1997 rask name : OPTIMA

Sample Height : $\quad 1.0000$ Solution Volume : $\quad 410.00$

on-Peak Integrations : 3 off-Peak Integrations : 1

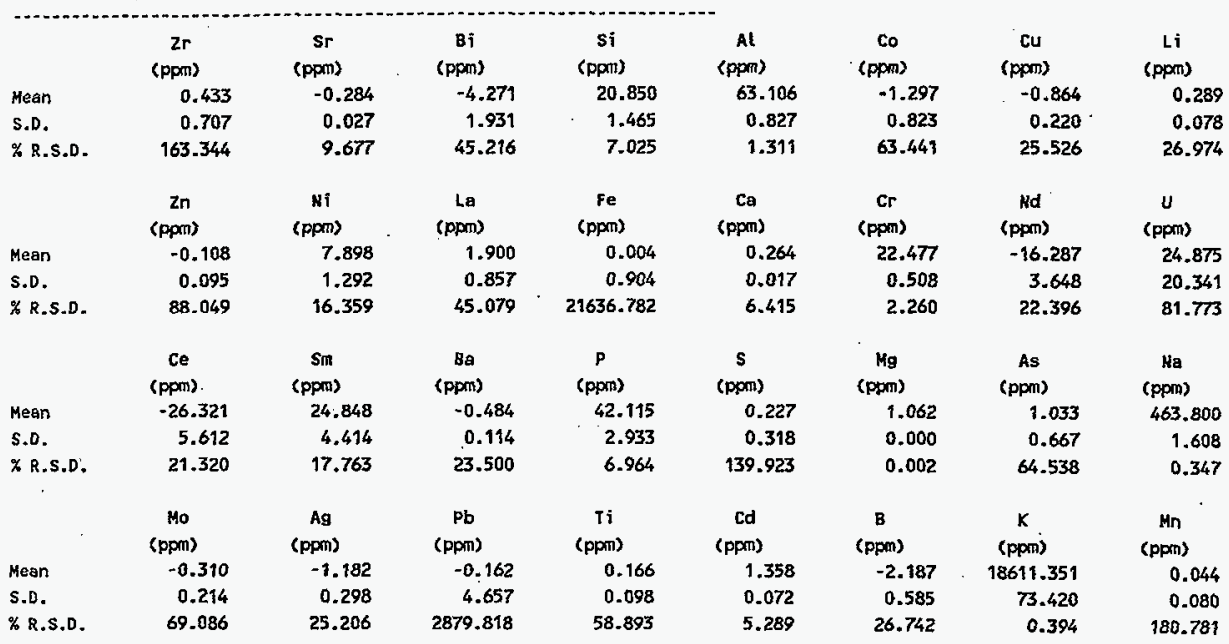

\begin{tabular}{lrrrr} 
& Sb & $V$ & \multicolumn{1}{c}{ Be } & \multicolumn{1}{c}{$T l$} \\
& (ppm) & \multicolumn{1}{c}{ (ppm) } & \multicolumn{1}{c}{ (ppm) } & \multicolumn{1}{c}{ (ppm) } \\
Mean & 1.650 & -1.885 & -0.214 & -35.882 \\
S.D. & 9.242 & 0.201 & 0.129 & 10.620 \\
\% R.S.D. & 560.093 & 10.655 & 60.491 & 29.596
\end{tabular}


Identity 1: \$95T001358_AX Identity 2: Fusion, .25-10-1-9 ml Task name : OPTIMA

Sample Neight :

1.0000 solution valume

on-Peak Integrations : 3

\begin{tabular}{|c|c|c|c|c|c|c|c|c|}
\hline & $\underset{(\mathrm{ppm})}{\mathrm{Zr}}$ & $\begin{array}{c}\mathrm{Sr} \\
(\mathrm{ppm})\end{array}$ & $\underset{\text { (ppprn) }}{\mathrm{B}_{\overline{1}}}$ & $\begin{array}{c}s i \\
\text { (ppm) }\end{array}$ & $\begin{array}{c}\text { Al } \\
\text { (ppon) }\end{array}$ & $\begin{array}{c}\text { co } \\
\text { (ppon) }\end{array}$ & $\underset{\text { (pprn) }}{C u}$ & $\underset{\text { (ppm) }}{\mathrm{Li}}$ \\
\hline Mean & 4009.527 & 4098.939 & 4117.237 & 4101.170 & 4144.569 & 3992.386 & 3947.948 & 4015.415 \\
\hline S.D. & 0.855 & 4.969 & 8.987 & 9.803 & 5.931 & 2.966 & 3.279 & 5.755 \\
\hline \% R.S.D. & 0.021 & 0.121 & 0.218 & $0.239^{\circ}$ & 0.143 & 0.074 & 0.083 & 0.143 \\
\hline
\end{tabular}

\begin{tabular}{|c|c|c|c|c|c|c|c|c|}
\hline . & $\begin{array}{c}\text { Zn } \\
\text { (ppm) }\end{array}$ & $\underset{\text { (ppm) }}{\mathrm{Ni}}$ & $\begin{array}{c}\text { La } \\
\text { (ppri) }\end{array}$ & $\begin{array}{c}F e \\
\text { (pprs) }\end{array}$ & $\begin{array}{c}\mathrm{Ca} \\
\text { (ppon) }\end{array}$ & $\begin{array}{c}\mathrm{Cr}_{\mathrm{r}} \\
(\mathrm{ppm})\end{array}$ & $\begin{array}{c}\text { Nd } \\
\text { (ppon) }\end{array}$ & $\underset{(\mathrm{ppm})}{U}$ \\
\hline Mean & 3930,638 & 4007.183 & 4053.604 & 3913.945 & 3998.499 & 3976.491 & 3994.956 & 7664.882 \\
\hline S.D. & 3.394 & 6.633 & 7.743 & 16.718 & 2.157 & 3.148 & 26.505 & 15.723 \\
\hline
\end{tabular}

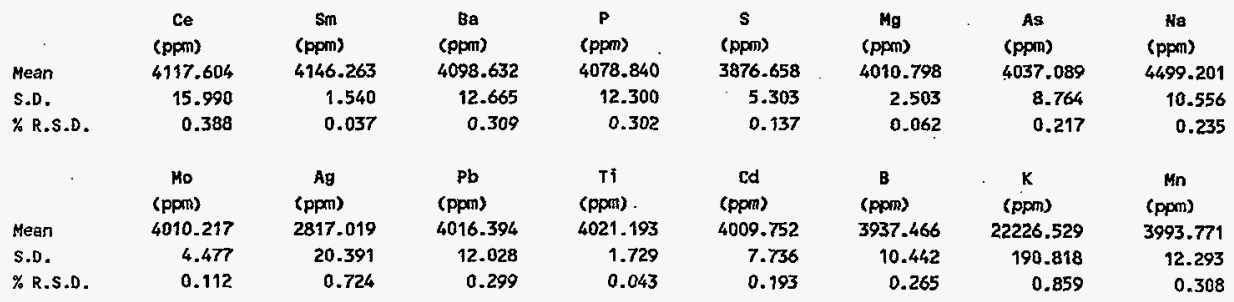

\begin{tabular}{lrrrr} 
& \multicolumn{1}{c}{ Sb } & \multicolumn{1}{c}{ Be } & \multicolumn{1}{c}{ Tl } \\
& \multicolumn{1}{c}{ (ppm) } & \multicolumn{1}{c}{ (ppm) } & \multicolumn{1}{c}{ (ppm) } & \multicolumn{1}{c}{ (ppm) } \\
Hean & 4002.281 & 4007.775 & 3995.981 & 4216.635 \\
S.D. & 23.264 & 5.603 & 6.452 & 18.226 \\
\% R.s.0. & 0.581 & 0.340 & 0.161 & 0.432
\end{tabular}




\section{HNF-SD-WM-DP-258, REV. 0}

Identity 1: CCV Task neme : OPT KMA

Sample Weight :

Identity 2: Quality Control

12:27 pK July 23, 1997

on-Peak Integrations : 3 off-Peak Integrations : 1

\begin{tabular}{|c|c|c|c|c|c|c|c|c|}
\hline & $\begin{array}{c}2 r \\
(p p m)\end{array}$ & $\begin{array}{c}\mathrm{Sr} \\
\text { (ppm) }\end{array}$ & $\begin{array}{c}B i \\
\text { (ppm) }\end{array}$ & $\begin{array}{c}\mathrm{Si} \\
\text { (ppm) }\end{array}$ & $\begin{array}{c}\text { AL } \\
\text { (PppD) }\end{array}$ & $\begin{array}{c}\text { Co } \\
\text { (ppm) }\end{array}$ & $\underset{\text { (ppm) }}{\mathrm{Cu}}$ & $\begin{array}{c}\text { Li } \\
\text { (ppm) }\end{array}$ \\
\hline Mean & 4.848 & 5.029 & 5.036 & 4.726 & 4.976 & 4.822 & 5.184 & 5.179 \\
\hline S.0. & 0.008 & 0.016 & 0.024 & 0.011 & 0.010 & 0.005 & 0.023 & 0.043 \\
\hline \multirow[t]{2}{*}{$\%$ R.S.D. } & 0.169 & 0.317 & 0.483 & 0.234 & 0.196 & 0.111 & 0.440 & 0.824 \\
\hline & $\begin{array}{c}\mathrm{Zn} \\
\text { (ppm) }\end{array}$ & $\begin{array}{c}\mathrm{Ni} \\
\text { (ppm) }\end{array}$ & $\begin{array}{c}\text { La } \\
\text { (ppm) }\end{array}$ & $\begin{array}{c}\mathrm{Fe} \\
\text { (ppon) }\end{array}$ & $\begin{array}{c}\mathrm{Ca} \\
\text { (ppm) }\end{array}$ & $\begin{array}{c}\mathrm{cr} \\
(\mathrm{ppm})\end{array}$ & $\begin{array}{c}\text { Nd } \\
\text { (ppon) }\end{array}$ & $\underset{\text { (ppM) }}{U}$ \\
\hline Mean & 4.889 & 4.915 & 5.078 & 4.882 & 4.884 & 4.863 & 5.384 & 9.462 \\
\hline S.0. & 0.012 & 0.014 & 0.012 & 0.029 & 0.016 & 0.012 & 0.027 & 0.018 \\
\hline \multirow[t]{2}{*}{$\%$ R.S.D. } & 0.254 & 0.278 & 0.232 & 0.599 & 0.322 & 0.242 & 0.502 & 0.185 \\
\hline & $\begin{array}{c}\mathrm{Ce} \\
\text { (ppm) }\end{array}$ & $\begin{array}{c}\text { Sm } \\
\text { (ppm) }\end{array}$ & $\begin{array}{c}\mathrm{Be} \\
\text { (ppm) }\end{array}$ & $\begin{array}{c}P \\
\langle p p m\rangle\end{array}$ & $\frac{\mathbf{s}}{(p p p)}$ & $\begin{array}{c}\mathrm{Mg} \\
(\mathrm{ppm})\end{array}$ & $\begin{array}{c}\text { As } \\
\text { (ppm) }\end{array}$ & $\begin{array}{c}\mathrm{Ne}_{0} \\
\text { (pppri) }\end{array}$ \\
\hline Mean & 4.991 & 5.171 & 5.100 & 4.896 & 4.696 & 4.728 & 5.079 & 4.989 \\
\hline S.D. & 0.022 & 0.016 & 0.020 & 0.040 & 0.014 & 0.007 & 0.021 & 0.029 \\
\hline \multirow[t]{2}{*}{ \% R.S.D. } & 0.448 & 0.314 & 0.389 & 0.814 & 0.296 & 0.157 & 0.419 & 0.585 \\
\hline & $\begin{array}{l}\text { Mo } \\
\text { (ppm) }\end{array}$ & $\begin{array}{c}\mathrm{Ag} \\
(\mathrm{ppm})\end{array}$ & $\begin{array}{c}\mathrm{Pb} \\
(\mathrm{ppm})\end{array}$ & $\begin{array}{c}T i \\
\text { (ppra) }\end{array}$ & $\begin{array}{c}\text { Cd } \\
\text { (ppm) }\end{array}$ & $\begin{array}{c}\text { B } \\
\text { (ppm) }\end{array}$ & $\begin{array}{c}K \\
\text { (ppm) }\end{array}$ & $\begin{array}{c}\text { Mn } \\
\text { (ppm) }\end{array}$ \\
\hline Mean & 4.861 & 5.091 & 4.951 & 4.788 & 4.949 & 4.955 & 4.837 & 4.915 \\
\hline S.D. & 0.007 & 0.007 & 0.019 & 0.011 & 0.008 & 0.021 & 0.061 & 0.006 \\
\hline * R.S.D. & 0.147 & 0.131 & 0.379 & 0.224 & 0.155 & 0.428 & 1.262 & 0.112 \\
\hline
\end{tabular}

\begin{tabular}{lrrrr} 
& Sb & \multicolumn{1}{c}{$\begin{array}{c}\text { Be } \\
\text { (ppm) }\end{array}$} & \multicolumn{1}{c}{$\begin{array}{c}\text { Tl } \\
\text { (ppm) }\end{array}$} & \multicolumn{1}{c}{$\begin{array}{c}\text { (ppm) } \\
\text { (ppm) }\end{array}$} \\
Mean & 4.702 & 4.864 & 5.144 & 5.085 \\
S.D. & 0.036 & 0.006 & 0.019 & 0.024 \\
\% R.S.D. & 0.772 & 0.127 & 0.370 & 0.470
\end{tabular}


Identity 1: CCB Identity 2: Quality. Control 12:31 PH July 23, 1997 rask name : opTIMA Sample Weight : 1.0000 Solution Volume : $\quad \mathbf{1 . 0 0}$ on-Peak Integrations : 3 off-Peak Integrations : 1

\begin{tabular}{|c|c|c|c|c|c|c|c|c|}
\hline & $\mathbf{Z r}$ & $\mathbf{s r}$ & $B i$ & $\mathbf{S} \mathfrak{i}$ & Al & Co & $\mathrm{Cu}$ & Li \\
\hline & $(\mathbf{p p}(\mathbf{n})$ & (ppal) & (ppn) & (ppra) & (ppos) & (ppm) & (ppm) & (ppm) \\
\hline Mean & -0.001 & 0.000 & -0.008 & -0.002 & 0.009 & 0.003 & 0.001 & 0.000 \\
\hline S.D. & 0.001 & 0.000 & 0.009 & 0.002 & 0.007 & 0.002 & 0.001 & 0.000 \\
\hline \multirow[t]{2}{*}{ \% R.S.D. } & 104.578 & 114.564 & 111.275 & 101.337 & 74.829 & 58.135 & 80.098 & 105.706 \\
\hline & $\underset{\text { (ppm) }}{2 n}$ & $\begin{array}{c}N i \\
\text { (ppm) }\end{array}$ & $\begin{array}{c}\text { La } \\
\text { (ppmi) }\end{array}$ & $\underset{\text { (pprn) }}{\mathrm{Fe}}$ & $\begin{array}{c}\text { Ca } \\
\text { (ppm) }\end{array}$ & $\begin{array}{c}\mathrm{Cr} \\
(\mathrm{pPn})\end{array}$ & $\begin{array}{c}\text { Nd } \\
\text { (ppm) }\end{array}$ & $\begin{array}{c}U \\
\text { (ppor) }\end{array}$ \\
\hline Mean & 0.000 & 0.003 & -0.000 & -0.002 & 0.002 & 0.000 & 0.001 & -0.069 \\
\hline S.D. & 0.000 & 0.002 & 0.001 & 0.002 & 0.000 & 0.003 & 0.006 & 0.040 \\
\hline \multirow[t]{2}{*}{ * R.S.D. } & 67.283 & 58.671 & 280.568 & 77.676 & 18.676 & 3930.080 & 421.105 & 57.940 \\
\hline & $\underset{\text { (ppm) }}{\mathrm{Ce}}$ & $\begin{array}{c}\text { sm } \\
\text { (ppm) }\end{array}$ & $\begin{array}{c}\mathrm{Ba} \\
(\mathrm{ppm})\end{array}$ & $\underset{(p p m)}{P}$ & $\begin{array}{c}\mathrm{s} \\
\text { (ppm) }\end{array}$ & $\begin{array}{c}\mathrm{Mg} \\
\text { (ppm) }\end{array}$ & $\begin{array}{c}\text { As } \\
(p p m)\end{array}$ & $\begin{array}{c}\mathrm{Na} \\
\text { (Ppm) }\end{array}$ \\
\hline Mean & 0.029 & 0.035 & 0.000 & -0.006 & -0.004 & -0.000 & 0.002 & 0.001 \\
\hline$S .0$ & 0.007 & 0.010 & 0.000 & 0.004 & 0.001 & 0.000 & 0.001 & 0.006 \\
\hline \multirow[t]{2}{*}{ \% R.S.D. } & 25.050 & 30.190 & 86.927 & 68.122 & 17.663 & 315.482 & 74.218 & 754.892 \\
\hline & $\begin{array}{c}\text { Mo } \\
\text { (ppm) }\end{array}$ & $\begin{array}{c}\text { Ag } \\
\text { (Ppm) }\end{array}$ & $\begin{array}{c}\mathrm{Pb} \\
\text { (ppm) }\end{array}$ & $\begin{array}{c}\mathrm{Ti} \\
(\mathrm{ppm})\end{array}$ & $\begin{array}{c}\text { cd } \\
\text { (ppm) }\end{array}$ & $\stackrel{\text { B }}{(p p m)}$ & $\underset{\text { (ppm) }}{K}$ & $\begin{array}{c}M n \\
\text { (ppom) }\end{array}$ \\
\hline Mean & -0.001 & 0.000 & 0.010 & -0.000 & 0.002 & -0.002 & -0.087 & 0.000 \\
\hline S.D. & 0.001 & 0.001 & 0.007 & 0.001 & 0.001 & 0.002 & 0.013 & 0.000 \\
\hline \multirow[t]{2}{*}{ \% R.S.D. } & 160.005 & 261.260 & 71.441 & 146.165 & 39.841 & 73.552 & 15.413 & 3346.546 \\
\hline & $\begin{array}{c}\text { Sb } \\
\text { (ppm) }\end{array}$ & $\begin{array}{c}v \\
\text { (ppm) }\end{array}$ & $\begin{array}{c}\mathrm{Be} \\
\text { (ppm) }\end{array}$ & $\begin{array}{c}T t \\
\text { (ppm) }\end{array}$ & & & & \\
\hline Mean & 0.050 & 0.000 & -0.001 & -0.044 & & & & \\
\hline S.D. & 0.020 & 0.000 & 0.000 & 0.027 & & & & \\
\hline X R.S.D. & 39.568 & 385.289 & 57.717 & 62.316 & & & & \\
\hline
\end{tabular}


HNF-SD-WM-DP-258, REV. 0

Identity 1: S95r001363_L Identity 2: Fusion, .25-10-2-8 ml 12:35 PH July 23, 1997 Task name : OPTIMA

Sample Weight : $\quad 1.0000$ solution volume : 205.00

on-Peak Integrations : 3 off-Peak Integrations : 1

\begin{tabular}{|c|c|c|c|c|c|c|c|c|}
\hline & $\begin{array}{c}2 r \\
\text { (ppm) }\end{array}$ & $\begin{array}{c}\mathrm{sr} \\
(\mathrm{ppm})\end{array}$ & $\begin{array}{c}B i \\
\text { (ppm) }\end{array}$ & $\begin{array}{c}\text { si } \\
(\mathrm{ppm})\end{array}$ & $\begin{array}{c}\text { AL } \\
\text { (ppom) }\end{array}$ & $\begin{array}{c}\text { Co } \\
\text { (ppom) }\end{array}$ & $\begin{array}{c}\mathrm{Cu} \\
\text { (Ppm) }\end{array}$ & $\begin{array}{c}\mathrm{Li} \\
\text { (ppom) }\end{array}$ \\
\hline Mean & 0.327 & 0.014 & $-2.47 t$ & 8.630 & 14.715 & 0.845 & 0.198 & 0.202 \\
\hline S.D. & 0.280 & 0.029 & 1.124 & 0.625 & 0.943 & 0.411 & 0.121 & 0.056 \\
\hline \multirow[t]{2}{*}{ \% R.S.D. } & 85.401 & 208.167 & 45.489 & 7.243 & 6.406 & 48.587 & 61.181 & 27.738 \\
\hline & $\begin{array}{c}\mathrm{Zn} \\
(p p+n)\end{array}$ & $\begin{array}{c}\mathrm{Ni} \\
\text { (ppm) }\end{array}$ & $\begin{array}{c}\text { Le } \\
\text { (ppm) }\end{array}$ & $\begin{array}{c}\mathrm{Fe} \\
\text { (ppm) }\end{array}$ & $\begin{array}{c}\mathrm{Ca} \\
\text { (Ppm) }\end{array}$ & $\underset{(\mathrm{ppm})}{\mathrm{Cr}}$ & $\begin{array}{c}\text { Hd } \\
\text { (ppon) }\end{array}$ & $\underset{\text { (ppm) }}{u}$ \\
\hline Hean & 0.136 & 7.276 & 0.192 & -1.324 & 0.244 & 3.828 & -3.862 & 7.178 \\
\hline S.D. & 0.076 & 0.764 & 0.288 & 0.306 & B.022 & 0.228 & 2.009 & 10.554 \\
\hline \multirow[t]{2}{*}{ * R.S.D. } & 55.486 & 10.501 & 150.068 & 23.140 & 9.165 & 5.956 & 52.009 & 147.031 \\
\hline & $\begin{array}{c}\mathrm{Ce} \\
\text { (pPD) }\end{array}$ & $\begin{array}{c}\mathrm{Sm} \\
(\mathrm{ppm})\end{array}$ & $\begin{array}{c}\text { Ba } \\
\text { (ppm) }\end{array}$ & $\begin{array}{c}P \\
\text { (ppm) }\end{array}$ & $\begin{array}{c}\mathrm{S} \\
(\mathrm{ppm})\end{array}$ & $\begin{array}{c}\mathrm{Mg} \\
\text { (ppn) }\end{array}$ & (ppm) & $\begin{array}{c}\mathrm{Ma} \\
(p p m)\end{array}$ \\
\hline Mean & 5.359 & 2.470 & 0.068 & 74.224 & -0.782 & 0.432 & 2.028 & 543.014 \\
\hline S.D. & 3.239 & 2.580 & 0.051 & 2.614 & 0.787 & 0.021 & 1.279 & 2.336 \\
\hline \multirow[t]{2}{*}{ \% R.S.D. } & 60.441 & 104.454 & 74.412 & 3.522 & 100.659 & 4.949 & 63.062 & 0.430 \\
\hline & (ppon) & $\begin{array}{c}\text { Ag } \\
\text { (ppm) }\end{array}$ & $\begin{array}{c}P b \\
\text { (pprn) }\end{array}$ & (ppm) & (ppm) & $\begin{array}{c}\text { B } \\
\text { (ppm) }\end{array}$ & $\begin{array}{c}\mathrm{K} \\
\text { (ppm) }\end{array}$ & $\begin{array}{c}\text { Mn } \\
\text { (ppm) }\end{array}$ \\
\hline Mean & 0.034 & 0.122 & 2.627 & 0.126 & 0.514 & -0.703 & 18227.396 & 0.118 \\
\hline S.D. & 0.133 & 0.225 & 1.367 & 0.069 & 0.282 & 0.168 & 61.350 & 0.071 \\
\hline \multirow[t]{2}{*}{ \% R.S.D. } & 398.161 & 184.806 & 52.033 & 54.988 & 54.896 & 23.890 & 0.337 & 59.881 \\
\hline & $\begin{array}{c}S b \\
\text { (ppm) }\end{array}$ & $\begin{array}{c}v \\
(\mathrm{ppm})\end{array}$ & $\begin{array}{c}\text { Be } \\
\text { (ppm) }\end{array}$ & $\begin{array}{c}\text { T1 } \\
\text { (ppm) }\end{array}$ & & & & \\
\hline Mean & -0.092 & -0.182 & -0.071 & 15.281 & & & & \\
\hline S.0. & 7.861 & 0.192 & 0.056 & 6.030 & & & & \\
\hline \% R.S.D. & 8574.964 & 105.300 & 78.153 & 39.462 & & & & \\
\hline
\end{tabular}




\section{HNF-SD-WM-DP-258, REV. 0}

Identity 1: \$95T001363 Identity 2: Fusion,-25-10 ml 12:38 PM July 23, 1997 rask name : OPTIMA

Sample Weight : $\quad 1.0000$ solution volume : $\quad \mathbf{4 1 . 0 0}$

on-Peak Integrations : 3 off-Peak Integrations : 1

\begin{tabular}{|c|c|c|c|c|c|c|c|c|}
\hline & $\begin{array}{c}2 r \\
\text { (ppm) }\end{array}$ & $\begin{array}{c}\mathrm{Sr} \\
\text { (ppm) }\end{array}$ & $\begin{array}{c}\mathrm{Bi} \\
\text { (ppm) }\end{array}$ & $\begin{array}{c}s i \\
(p p m)\end{array}$ & $\underset{\text { (PPR) }}{\text { Al }}$ & $\begin{array}{c}\text { Co } \\
\text { (ppm) }\end{array}$ & $\underset{\text { (ppon) }}{\mathrm{Cu}}$ & $\begin{array}{c}\mathrm{Li} \\
\text { (ppon) }\end{array}$ \\
\hline Mean & 0.112 & -0.020 & -0.078 & 4.377 & 13.990 & -0.013 & -0.045 & -0.021 \\
\hline S.D. & 0.144 & 0.013 & 0.450 & 0.358 & 0.336 & 0.082 & 0.048 & 0.029 \\
\hline \multirow[t]{2}{*}{ \% R.S.D. } & 129.019 & 62.490 & 579.215 & 8.175 & 2.405 & 758.423 & 106.214 & 142.394 \\
\hline & $\underset{\text { Sppm) }}{2 n}$ & $\begin{array}{c}\text { Hi } \\
\text { (ppm) }\end{array}$ & $\begin{array}{c}\text { La } \\
\text { (ppon) }\end{array}$ & $\begin{array}{c}\mathrm{Fe} \\
(\mathrm{ppm})\end{array}$ & $\begin{array}{c}\mathrm{Ca} \\
\text { (ppan) }\end{array}$ & $\begin{array}{c}\mathrm{Cr} \\
\text { (ppom) }\end{array}$ & $\begin{array}{c}\text { Nd } \\
(\mathrm{ppm})\end{array}$ & $\begin{array}{c}U \\
\text { (ppm) }\end{array}$ \\
\hline Mean & 0.029 & 6.386 & 0.134 & -0.023 & 0.344 & 3.609 & -0.912 & 3.541 \\
\hline S.D. & 0.012 & 0.214 & 0.134 & 0.094 & 0.008 & 0.028 & 0.500 & 6.287 \\
\hline \multirow[t]{2}{*}{ * R.S.D. } & 41.186 & 3.347 & 99.813 & 407.870 & 2.255 & 0.787 & 54.759 & 177.523 \\
\hline & $\begin{array}{c}\mathrm{Ce} \\
\text { (ppm) }\end{array}$ & $\begin{array}{c}\text { Sin } \\
\text { (ppm) }\end{array}$ & $\begin{array}{c}\text { Ba } \\
\text { (ppm) }\end{array}$ & $\begin{array}{c}P \\
\text { (ppm) }\end{array}$ & $\begin{array}{c}S \\
\text { (pprol })\end{array}$ & $\begin{array}{c}\mathrm{Hg} \\
\text { (ppom) }\end{array}$ & $\begin{array}{c}\text { As } \\
\text { (ppm) }\end{array}$ & $\begin{array}{c}\mathrm{Na} \\
(\mathrm{pp} n)\end{array}$ \\
\hline Hean & -2.463 & 1.499 & -0.038 & 73.223 & -0.324 & 0.183 & -0.014 & 537.693 \\
\hline S.D. & 1.524 & 1.133 & 0.028 & 1.201 & 0.122 & 0.004 & 0.151 & 7.361 \\
\hline \multirow[t]{2}{*}{ \% R.S.D. } & 61.869 & 75.556 & 74.474 & 1.640 & 37.579 & 2.342 & 1110.763 & 1.369 \\
\hline & $\begin{array}{c}\text { Mo } \\
\text { (ppm) }\end{array}$ & $\begin{array}{c}\text { Ag } \\
\text { (ppm) }\end{array}$ & $\begin{array}{c}\mathrm{Pb} \\
(\mathrm{ppm})\end{array}$ & $\begin{array}{c}T i \\
\text { (ppon) }\end{array}$ & $\begin{array}{c}\mathrm{Cd} \\
(\mathrm{ppm})\end{array}$ & $\begin{array}{c}\text { B } \\
\text { (ppm) }\end{array}$ & $\begin{array}{c}K \\
(\mathrm{ppm})\end{array}$ & $\begin{array}{c}\text { Mn } \\
\text { (ppm) }\end{array}$ \\
\hline Mean & -0.113 & -0.147 & 0.282 & 0.066 & 0.054 & -0.196 & 17556.667 & 0.075 \\
\hline S.D. & 0.027 & 0.107 & 0.121 & 0.048 & 0.021 & 0.014 & 237.042 & 0.016 \\
\hline X R.s.D. & 23.560 & 72.280 & 43.019 & 72.471 & 40.115 & 7.121 & 1.350 & 21.121 \\
\hline
\end{tabular}

Mean

S.D.

Sb

(ppm)

0.348

1.704

489.304
$V$
(Ppm)
-0.11

0. 111

95.856

\begin{tabular}{cc} 
Be & \multicolumn{1}{c}{ Tl } \\
(ppm) & \multicolumn{1}{c}{ (ppm) } \\
-0.021 & -3.205 \\
0.007 & 3.790 \\
30.889 & 118.224
\end{tabular}


HNF-SD-WM-DP-258, REV. 0

Identity 1: \$95T001363_D Identity 2: Fusion, .25-10 ml

12:41 PN July 23, 1997

Task name : OPTIMA

Sample Keight : $\quad 1.0000$ solution Volume : $\quad 41.00$

on-Peak Integrations : 3 off-Peak Integrations : 1

\begin{tabular}{|c|c|c|c|c|c|c|c|c|}
\hline & $\underset{(\mathrm{ppm})}{\mathrm{zr}}$ & $\begin{array}{c}S r \\
(p p m)\end{array}$ & $\begin{array}{c}\mathbf{B i} \\
\text { (ppm) }\end{array}$ & $\underset{\text { (ppm) }}{s i}$ & $\begin{array}{c}\text { Al } \\
\text { (ppm) }\end{array}$ & $\underset{(p p m)}{c o}$ & $\begin{array}{c}\mathrm{Cu} \\
\text { (ppm) }\end{array}$ & $\underset{\text { (ppm) }}{\mathrm{Li}}$ \\
\hline Hean & -0.012 & -0.008 & 0.242 & 3.624 & 14.510 & 0.090 & 0.002 & 0.014 \\
\hline S.D. & 0.066 & .0 .004 & 0.169 & 0.085 & 0.232 & 0.000 & 0.026 & 0.014 \\
\hline \multirow[t]{2}{*}{ \% R.S.D. } & 542.295 & 50.918 & 69.668 & 2.332 & 1.598 & 0.345 & 1274.852 & 99.147 \\
\hline & $\underset{\text { (ppm) }}{\text { Zn }}$ & $\begin{array}{c}\text { Ni } \\
\text { (ppm) }\end{array}$ & $\begin{array}{c}\text { La } \\
\text { (ppm) }\end{array}$ & $\begin{array}{c}F e \\
(p p m)\end{array}$ & $\begin{array}{c}\mathrm{Ca} \\
(\mathrm{ppm})\end{array}$ & $\underset{(p p m)}{c r}$ & $\begin{array}{c}\text { Nd } \\
\text { (ppm) }\end{array}$ & $\begin{array}{c}U \\
(\mathrm{ppm})\end{array}$ \\
\hline Mean & 0.033 & 4.670 & 0.018 & -0.233 & 0.285 & 3.648 & -0.728 & -1.777 \\
\hline S.D. & 0.018 & 0.304 & 0.033 & 0.167 & 0.000 & 0.120 & 0.224 & 1.497 \\
\hline \multirow[t]{2}{*}{ \% R.S.D. } & 54.124 & 6.516 & 384.847 & 71.836 & 0.000 & 3.296 & $30: 768$ & 84.279 \\
\hline & $\begin{array}{c}\mathrm{Ce} \\
\text { (ppm) }\end{array}$ & $\begin{array}{c}\text { Sm } \\
\text { (ppm) }\end{array}$ & $\underset{\text { (ppm) }}{\text { Ba }}$ & $\underset{\text { (ppm) }}{p}$ & $\begin{array}{c}s \\
\text { (ppon) }\end{array}$ & $\begin{array}{c}\mathrm{Hg} \\
\text { (ppom) }\end{array}$ & $\begin{array}{c}\text { As } \\
\text { (ppm) }\end{array}$ & $\underset{\text { (ppon) }}{\mathrm{NB}}$ \\
\hline Meen & -1.131 & 0.872 & -0.010 & 87.909 & -0.276 & 0.166 & 0.101 & 567.118 \\
\hline S.D. & 0.586 & 0.353 & 0.010 & 0.435 & 0.312 & 0.000 & 0.125 & 1.905 \\
\hline X R.S.D. & 51.834 & 40.463 & 109.389 & 0.495 & 113.109 & 0.001 & 123.624 & 0.336 \\
\hline & $\begin{array}{c}\text { Mo } \\
\text { (ppri) }\end{array}$ & $\begin{array}{c}\text { Ag } \\
\text { (ppm) }\end{array}$ & $\begin{array}{c}\mathrm{Pb} \\
(\mathrm{ppm})\end{array}$ & $\begin{array}{c}T i \\
\text { (ppm) }\end{array}$ & $\begin{array}{c}\text { cd } \\
\text { (ppm) }\end{array}$ & $\begin{array}{c}B \\
\text { (ppon) }\end{array}$ & $\underset{\text { (pprt) }}{k}$ & $\begin{array}{c}\text { Hn } \\
\text { (ppm) }\end{array}$ \\
\hline Mean & -0.060 & -0.033 & 0.074 & 0.027 & 0.086 & -0.296 & 17264.525 & 0.019 \\
\hline S.D. & 0.042 & 0.026 & 0.384 & 0.029 & 0.026 & 0.017 & 30.568 & 0.008 \\
\hline \multirow[t]{2}{*}{ \%R.S.D. } & 69.561 & 78.514 & 520.587 & 106.445 & 29.736 & 5.908 & 0.177 & 43.379 \\
\hline & $\begin{array}{c}\mathrm{Sb} \\
\text { (ppm) }\end{array}$ & $\begin{array}{c}v \\
\text { (ppm) }\end{array}$ & $\begin{array}{c}\mathrm{Be} \\
\text { (ppn) }\end{array}$ & $\underset{(p p m)}{T l}$ & & & & \\
\hline Mean & 1.027 & 0.016 & -0.044 & -4.857 & & & & \\
\hline S.D. & 0.582 & 0.008 & 0.007 & 1.049 & & & & \\
\hline \% R.S.D. & 56.695 & 52.002 & 14.889 & 21.591 & & & & \\
\hline
\end{tabular}


Identity 1: S95T001363_A Identity 2: Fusion, .25-10.mL 12:44 PN July 23, 1997 Task narre : OPTIMA

Sample Height : $\quad 1.0000$ solution Volume : $\quad 41.00$

On-Peak Integrations : 3 off-Peak Integrations : 1

\begin{tabular}{|c|c|c|c|c|c|c|c|c|}
\hline & $\mathbf{z r}$ & Sr & $\mathrm{Bi}$ & si & Al & Co & cu. & LI \\
\hline & (ppm) & (ppm) & (ppm) & (ppm) & (ppen) & (ppon) & (ppm) & $(\mathrm{ppm})$ \\
\hline Mean & 39.879 & 41.529 & 41.335 & 43.282 & 56.272 & 39.819 & 42.918 & 40.265 \\
\hline S.D. & 0.026 & 0.263 & 0.322 & 0.260 & 0.368 & 0.296 & 0.389 & 0.579 \\
\hline \multirow[t]{3}{*}{ \% R.S.D. } & 0.064 & 0.634 & 0.780 & 0.601 & 0.654 & 0.744 & 0.907 & 1.437 \\
\hline & $\mathrm{Zn}$ & $\mathrm{Mi}$ & Le & $\mathrm{Fe}$ & $\mathrm{Ca}$ & $\mathrm{Cr}$ & Md & u \\
\hline & (ppm) & (ppm) & (ppm) & (ppm) & (PPm) & (ppm) & (ppm) & (ppm) \\
\hline Hean & 39.707 & 46.411 & 41.998 & 39.499 & 39.859 & 43.587 & 43.777 & 68.902 \\
\hline s.o. & 0.159 & 0.203 & 0.314 & 0.165 & 0.273 & 0.319 & 0.665 & 3.418 \\
\hline \multirow[t]{3}{*}{ \% R.S.D. } & 0.401 & 0.438 & 0.748 & 0.418 & 0.684 & 0.732 & 1.519 & 4.961 \\
\hline & $\mathrm{Ce}$ & Sm & $\mathrm{Ba}$ & p & $\mathbf{s}$ & $\mathbf{M g}$ & As & Me \\
\hline & (ppm) & (ppm) & (ppon) & (ppn) & (ppn) & (ppm) & (ppm) & (ppm) \\
\hline Mean & 41.289 & 42.592 & 41.933 & 113.956 & 38.012 & 37.949 & 41.386 & 606.184 \\
\hline S.D. & 0.847 & 0.461 & 0.330 & 1.957 & 0.486 & 0.069 & 0.188 & 8.120 \\
\hline \multirow[t]{3}{*}{ * R.S.D. } & 2.052 & 1.083 & 0.788 & 1.718 & 1.278 & 0.181 & 0.454 & 1.340 \\
\hline & Mo & $\mathbf{A g}$ & $\mathbf{P b}$ & Ti & Cd & B & K & Mn \\
\hline & (ppm) & (ppm) & (ppon) & (ppm) & (ppm) & (pptn) & (ppom) & (ppm) \\
\hline Mean & 39.826 & 31.926 & 42.288 & 39.439 & 40.372 & 39.186 & 18286.573 & 40.465 \\
\hline S.D. & 0.166 & 0.056 & 0.818 & 0.098 & 0.084 & 0.293 & 214.062 & 0.103 \\
\hline \% R.S.D. & 0.417 & 0.176 & 1.934 & 0.248 & 0.208 & 0.748 & 1.171 & 0.256 \\
\hline
\end{tabular}

\begin{tabular}{lrrrr} 
& sb & \multicolumn{1}{c}{$v$} & \multicolumn{1}{c}{ Be } & \multicolumn{1}{c}{$T$} \\
& (ppm) & \multicolumn{1}{c}{ (ppm) } & \multicolumn{1}{c}{ (ppm) } & \multicolumn{1}{c}{ (ppm) } \\
Mean & 36.466 & .40 .293 & 40.939 & 38.973 \\
S.D. & 1.536 & 0.048 & 0.168 & 2.559 \\
\% R.S.D. & 4.213 & 0.119 & 0.411 & 6.565
\end{tabular}


HNF-SD-WM-DP-258, REV. 0

Identity 1: $\$ 957001363$ X Identity 2: Fusion, .25-10-1-9mt Task name : OPYIMA

Sample Weight :

1.0000 solution volume : $\quad \mathbf{4 1 0 . 0 0}$

on-Peak Integrations : 3 off-Peak Integrations : 1

\begin{tabular}{|c|c|c|c|c|c|c|c|c|}
\hline & $\begin{array}{c}2 r \\
(p p m)\end{array}$ & $\begin{array}{c}\text { Sr } \\
\text { (ppom) }\end{array}$ & $\begin{array}{c}B i \\
\text { (Ppm) }\end{array}$ & $\begin{array}{c}\mathrm{si} \\
(\mathrm{ppm})\end{array}$ & $\begin{array}{c}\text { Al } \\
\text { (ppm) }\end{array}$ & $\begin{array}{c}\text { Co } \\
\text { (ppm) }\end{array}$ & $\begin{array}{c}\mathrm{Cu} \\
\text { (pppn) }\end{array}$ & $\underset{\text { (ppom) }}{\text { Li }}$ \\
\hline Mean & 0.127 & -0.018 & -0.815 & 11.255 & 12.968 & 2.979 & 0.191 & 0.021 \\
\hline s.o. & 0.710 & 0.069 & 3.756 & 1.294 & 4.296 & 0.821 & 0.203 & 0.156 \\
\hline \multirow[t]{2}{*}{ * R.S.D. } & 559.023 & 377.492 & 460.823 & 11.498 & 33.128 & 27.547 & 108.285 & 755.779 \\
\hline & $\begin{array}{c}\text { Zn } \\
\text { (ppri) }\end{array}$ & $\underset{\text { (ppm) }}{\mathrm{Ni}}$ & $\begin{array}{c}\text { La } \\
\text { (ppra) }\end{array}$ & $\underset{\text { (pprn) }}{\text { Fe }}$ & $\begin{array}{c}\text { Ca } \\
\text { (ppon) }\end{array}$ & $\begin{array}{c}\mathrm{Cr} \\
\text { (ppm) }\end{array}$ & $\begin{array}{c}\text { Nd } \\
\text { (ppm) }\end{array}$ & $\begin{array}{c}\mathrm{U} \\
\text { (ppm) }\end{array}$ \\
\hline Mean & -0.061 & 6.795 & 0.752 & -2.717 & -0.958 & 3.688 & -2.409 & -6.460 \\
\hline s.o. & 0.026 & 0.464 & 0.332 & 0.824 & 0.000 & 1.081 & 6.345 & 10.363 \\
\hline \multirow[t]{2}{*}{ \% R.S.D. } & 42.248 & 6.827 & 44.136 & 30.334 & 0.000 & 29.308 & 263.427 & 160.414 \\
\hline & $\begin{array}{c}\text { Ce } \\
\text { (ppon) }\end{array}$ & $\begin{array}{c}\mathrm{Sm} \\
(\mathrm{ppm})\end{array}$ & $\begin{array}{c}\mathrm{Ba} \\
\text { (Ppm) }\end{array}$ & $\begin{array}{c}P \\
\text { (ppm) }\end{array}$ & $\begin{array}{c}s \\
\text { (ppm) }\end{array}$ & $\begin{array}{c}\text { Mg } \\
\text { (pporin) }\end{array}$ & $\begin{array}{c}\text { As } \\
\text { (ppm) }\end{array}$ & $\begin{array}{c}\mathrm{Na} \\
\text { (ppm) }\end{array}$ \\
\hline Mean & 2.298 & 20.755 & 0.008 & 70.966 & -2.629 & 0.593 & 4.679 & 553.534 \\
\hline S.D. & 5.851 & 3.880 & 0.080 & 3.133 & 1.147 & 0.043 & 0.668 & 2.336 \\
\hline \multirow[t]{2}{*}{ \% R.S.D. } & 254.678 & 18.692 & 964.365 & 4.415 & 43.612 & 7.218 & 14.278 & 0.422 \\
\hline & $\begin{array}{c}\text { Ho } \\
\text { (ppm) }\end{array}$ & $\begin{array}{c}\mathrm{Ag} \\
(\mathrm{pPm})\end{array}$ & $\begin{array}{c}\mathrm{Pb} \\
\text { (ppm) }\end{array}$ & $\begin{array}{c}T i \\
\text { (ppm) }\end{array}$ & $\begin{array}{c}\text { cd } \\
\text { (ppm) }\end{array}$ & $\begin{array}{c}\text { B } \\
\text { (ppm) }\end{array}$ & $\begin{array}{c}K \\
(\mathrm{ppm})\end{array}$ & $\begin{array}{c}\text { Hn } \\
\text { (ppm) }\end{array}$ \\
\hline Mean & 0.200 & 0.138 & 7.237 & 0.020 & 1.275 & -1.381 & 18694.328 & 0.240 \\
\hline S.D. & 0.657 & 0.249 & 6.835 & 0.070 & 0.247 & 0.180 & 161.477 & 0.142 \\
\hline \% R.S.D. & 328.050 & 181.052 & 94.441 & 357.269 & 19.332 & 73.044 & 0.864 & 59.038 \\
\hline
\end{tabular}

\begin{tabular}{|c|c|c|c|c|}
\hline & $\begin{array}{c}\text { sb } \\
\text { (ppm) }\end{array}$ & $\begin{array}{c}v \\
(\mathrm{ppm})\end{array}$ & $\begin{array}{c}\text { Be } \\
\text { (ppm) }\end{array}$ & $\begin{array}{c}\text { Tl } \\
\text { (ppm) }\end{array}$ \\
\hline Mean & 14.117 & -0.530 & -0.219 & 3.971 \\
\hline S.D. & 6.343 & 0.473 & 0.128 & 13.696 \\
\hline \% R.S.D. & 44.932 & 89.128 & 58.256 & 344.896 \\
\hline
\end{tabular}


Identity 1: S95T001363_AX Identity 2: Fusion, .25-10-1-9mL Task name : OPTIKA Sample Height : $\quad 1.0000$ solution volume : 410.00 on-Peak Integrations : 3 off-Peak Integrations : 1

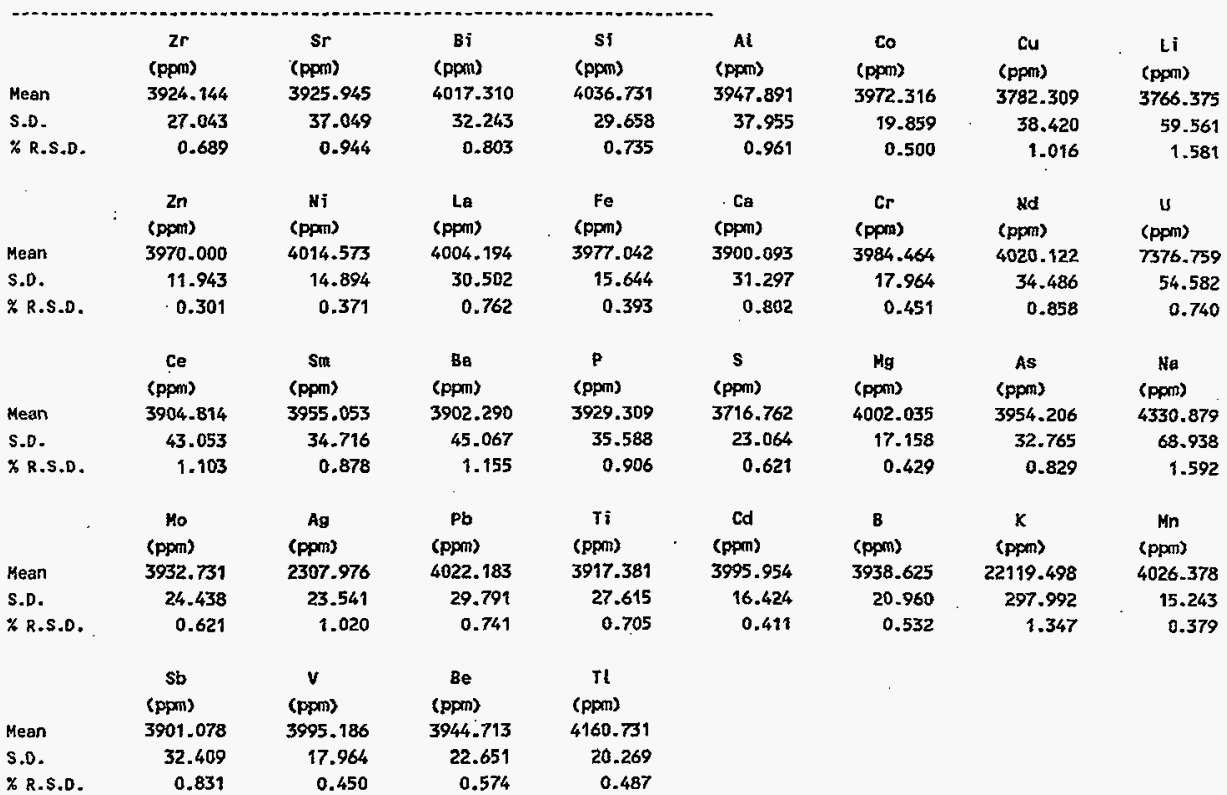


Identity 1: ICSA Identity 2: Quality Control Task name : OPIIMA Sample Weight : 1.0000 solution volume:

On-Peak Integrations : 3 off-Peak Integrations : 1

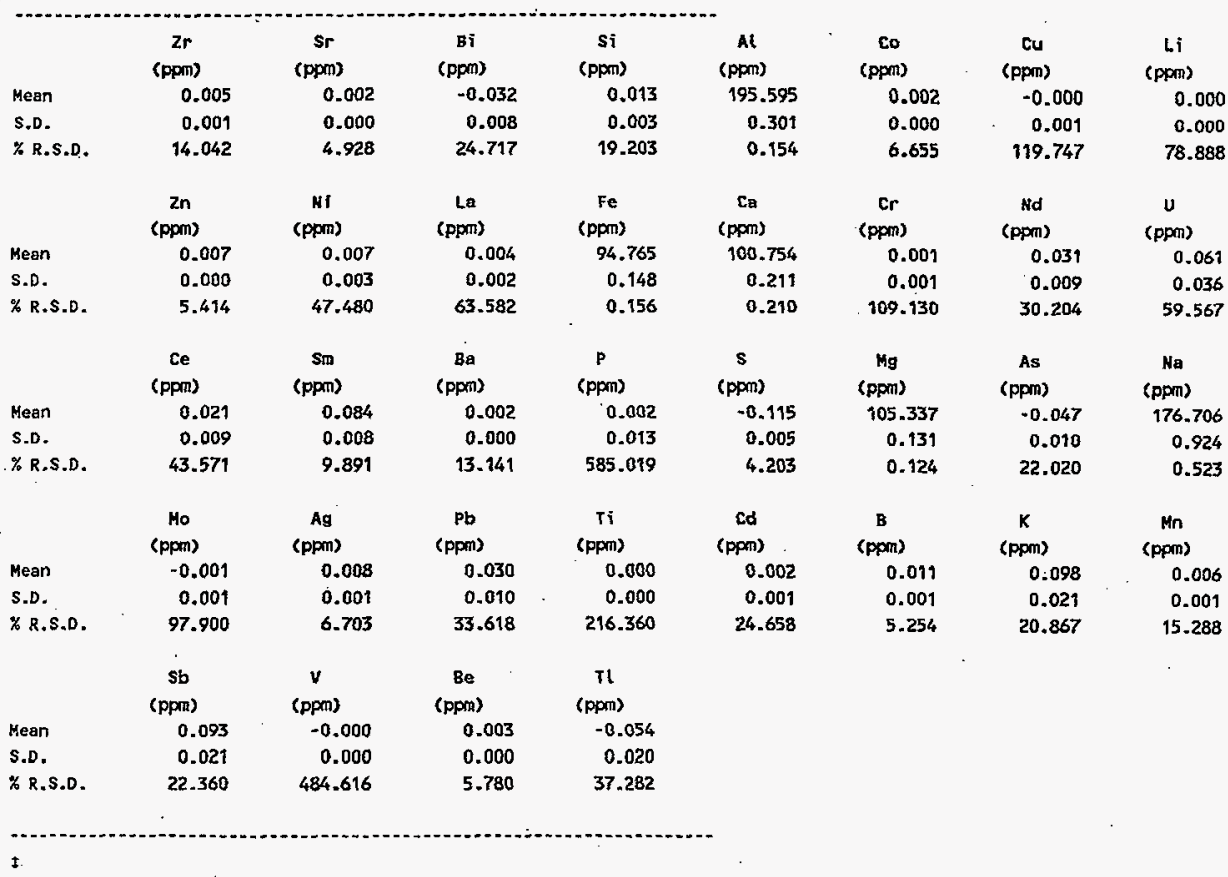


Identity 1: JCSAB Identity 2: Quality Control

1:23 PH July 23, 1997

Task name : OPJIMA

Sample Weight :

3.0000 Solution volume:

1.00

on-Peak Integrations : 3 off-Peak Integrations : 1

\begin{tabular}{|c|c|c|c|c|c|c|c|c|}
\hline . & $\begin{array}{c}\mathrm{Zr} \\
\text { (ppras) }\end{array}$ & $\begin{array}{c}\mathbf{s r} \\
\text { (Ppm) }\end{array}$ & $\begin{array}{c}\text { Bi } \\
\text { (ppm) }\end{array}$ & $\begin{array}{c}\text { Si } \\
\text { (ppmin) }\end{array}$ & $\begin{array}{c}\text { Al } \\
\text { (ppm) }\end{array}$ & $\begin{array}{c}\text { Co } \\
\text { (ppm) }\end{array}$ & $\begin{array}{c}\mathrm{Cu} \\
\text { (ppm) }\end{array}$ & $\underset{\text { (ppm) }}{\mathrm{Li}}$ \\
\hline Mean & -0.001 & 0.002 & -0.020 & 0.009 & 201.434 & 0.494 & 0.489 & 0.932 \\
\hline S.D. & 0.003 & 0.000 & 0.009 & 0.008 & 1.525 & 0.002 & 0.006 & 0.018 \\
\hline \multirow[t]{2}{*}{ \% R.S.D. } & 235.355 & 22.311 & 45.915 & 82.007 & 0.757 & 0.453 & 1.291 & 1.946 \\
\hline & $\begin{array}{c}\mathrm{Zn} \\
\text { (ppm) }\end{array}$ & $\begin{array}{c}\mathrm{Ni} \\
\text { (ppm) }\end{array}$ & $\begin{array}{c}\text { La } \\
\text { (ppm) }\end{array}$ & $\begin{array}{c}F e \\
\text { (ppm) }\end{array}$ & $\begin{array}{c}\mathrm{Ca} \\
\text { (ppm) }\end{array}$ & $\begin{array}{c}\mathrm{Cr} \\
\text { (ppm) }\end{array}$ & $\begin{array}{c}\text { Nd } \\
\text { (ppm) }\end{array}$ & $\begin{array}{c}u \\
\text { (Ppm) }\end{array}$ \\
\hline Mean & 0.976 & 0.977 & -0.001 & 94.087 & 101.723 & 0.488 & 0.010 & 0.027 \\
\hline S.D. & 0.003 & 0.003 & 0.004 & 0.353 & 0.569 & 0.008 & 0.011 & 0.146 \\
\hline \multirow[t]{2}{*}{ \% R.S.D. } & 0.322 & 0.318 & 308.005 & 0.375 & 0.560 & 1.564 & 115.334 & 545.042 \\
\hline & $\begin{array}{c}\text { Ce } \\
\text { (ppm) }\end{array}$ & $\begin{array}{c}\text { Sin } \\
\text { (ppm) }\end{array}$ & $\begin{array}{c}\mathrm{Ba} \\
(\mathrm{ppm})\end{array}$ & $\begin{array}{c}P \\
\text { (ppm) }\end{array}$ & $\stackrel{s}{(p p m)}$ & $\underset{\text { (ppm) }}{\mathrm{Mg}}$ & $\begin{array}{c}\text { As } \\
\text { (ppm) }\end{array}$ & $\begin{array}{c}\text { Na } \\
\text { (ppm) }\end{array}$ \\
\hline Mean & -0.028 & 0.073 & 0.505 & 0.010 & -0.130 & 104.589 & -0.049 & 185.955 \\
\hline S.D. & 0.035 & 0.030 & 0.006 & 0.008 & 0.008 & 0.428 & 0.007 & 3.308 \\
\hline \multirow[t]{2}{*}{ \% R.S.D. } & 125.798 & 41.892 & 1.121 & 78.414 & 6.060 & 0.410 & 13.351 & 1.779 \\
\hline & $\begin{array}{c}\text { Mo } \\
\text { (ppm) }\end{array}$ & $\begin{array}{c}\mathrm{Ag} \\
\text { (ppm) }\end{array}$ & $\begin{array}{c}\mathrm{Pb} \\
\text { (ppm) }\end{array}$ & $\begin{array}{c}\text { Ti } \\
(\mathrm{ppm})\end{array}$ & $\begin{array}{c}\text { Cd } \\
\text { (ppqm) }\end{array}$ & $\begin{array}{c}\text { B } \\
\text { (ppm) }\end{array}$ & $\underset{(p p m)}{K}$ & $\begin{array}{c}\text { Mn } \\
\text { (ppm) }\end{array}$ \\
\hline Mean & -0.005 & 1.031 & 1.002 & -0.000 & 0.980 & 0.008 & -0.063 & 0.494 \\
\hline S.0. & 0.002 & 0.082 & 0.023 & 0.001 & 0.006 & 0.001 & 0.035 & 0.002 \\
\hline \multirow[t]{2}{*}{ \% R.S.D. } & 39.059 & 0.234 & 2.321 & 414.877 & 0.616 & 15.040 & 56.368 & 0.333 \\
\hline & $\begin{array}{c}\text { sb } \\
\text { (ppm) }\end{array}$ & $\underset{\text { (ppin) }}{v}$ & $\begin{array}{c}\mathrm{Be} \\
(\mathrm{ppm})\end{array}$ & $\begin{array}{c}\text { Tl } \\
\text { (ppm) }\end{array}$ & & . & & \\
\hline Mean & 0.019 & 0.481 & 0.489 & -0.151 & & & & \\
\hline S.D. & 0.029 & 0.002 & 0.004 & 0.081 & & & & \\
\hline \% R.S.D. & 152.585 & 0.491 & 0.796 & 53.481 & & & & \\
\hline
\end{tabular}


Identity 1: cCV . Identity 2 : auality control

1:28 PM suly 23, 1997

Task name : OPTiMA

Sample Veight : $\quad 1.0000$ Solution Volume: $\quad 1.00$

on-Peak Integrations : 3 off-Peak Integrations : 1

\begin{tabular}{|c|c|c|c|c|c|c|c|c|}
\hline & $\begin{array}{c}\mathrm{Zr} \\
(\mathrm{gporn})\end{array}$ & $\begin{array}{c}\mathrm{sr} \\
\text { (ppm) }\end{array}$ & $\underset{(p p m)}{B i}$ & $\begin{array}{c}\mathrm{si} \\
(\mathrm{ppm})\end{array}$ & $\begin{array}{c}\mathrm{Al} \\
\text { (ppm) }\end{array}$ & $\begin{array}{c}\text { Co } \\
\text { (Ppm) }\end{array}$ & $\begin{array}{c}\mathrm{Cu} \\
\text { (ppon) }\end{array}$ & $\underset{(p p m)}{L i}$ \\
\hline Mean & 4.902 & 5.123 & 5.104 & 4.761 & 5.055 & 4.842 & 5.282 & 5.286 \\
\hline S.D. & 0.010 & 0.017 & 0.007 & 0.005 & 0.009 & 0.019 & 0.017 & 0.035 \\
\hline \multirow[t]{2}{*}{ X R.S.D. } & 0.197 & 0.326 & 0.130 & 0.103 & 0.175 & 0.398 & 0.328 & 0.663 \\
\hline & $\begin{array}{c}\mathrm{Zn} \\
(\mathrm{ppm})\end{array}$ & $\underset{\text { (ppm) }}{\mathrm{Ni}}$ & $\begin{array}{c}\text { La } \\
\text { (ppm) }\end{array}$ & $\begin{array}{c}\mathrm{Fe} \\
(p p \times(1)\end{array}$ & $\begin{array}{c}\mathrm{Ca} \\
(\mathrm{ppm})\end{array}$ & $\underset{\text { (ppm) }}{\mathrm{Cr}}$ & $\begin{array}{c}\text { Nd } \\
\text { (ppm) }\end{array}$ & $\underset{\text { (ppon) }}{U}$ \\
\hline Mean & 4.894 & 4.925 & 5.119 & 4.892 & 4.940 & 4.870 & 5.390 & 9.472 \\
\hline S.D. & 0.005 & 0.007 & 0.008 & 0.008 & 0.012 & 0.008 & 0.019 & 0.025 \\
\hline \multirow[t]{2}{*}{ \% R.s.D. } & 0.103 & 0.134 & 0.165 & 0.173 & 0.240 & 0.165 & 0.346 & 0.260 \\
\hline & $\begin{array}{c}\text { Ce } \\
\text { (ppm) }\end{array}$ & $\begin{array}{c}\mathrm{Sm} \\
\text { (pprin) }\end{array}$ & $\begin{array}{c}\text { Ba } \\
\text { (ppm) }\end{array}$ & $\begin{array}{c}P \\
\text { (ppm) }\end{array}$ & $\begin{array}{c}\text { S } \\
\text { (ppm) }\end{array}$ & $\underset{\text { (ppra) }}{\mathrm{Mg}}$ & $\begin{array}{c}\text { As } \\
\text { (ppm) }\end{array}$ & $\underset{\text { (ppom) }}{\mathrm{Na}}$ \\
\hline Mean & 5.056 & 5.278 & 5.203 & 4.973 & 4.750 & 4.745 & 5.139 & 5.081 \\
\hline S.D. & 0.017 & 0.016 & 0.019 & 0.021 & 0.012 & 0.005 & 0.016 & 0.032 \\
\hline \% R.S.D. & 0.332 & 0.303 & 0.358 & 0.430 & 0.257 & 0.106 & 0.315 & 0.632 \\
\hline . & $\begin{array}{c}\text { Mo } \\
\text { (ppm) }\end{array}$ & $\begin{array}{c}\mathrm{Ag} \\
(\mathrm{ppm})\end{array}$ & $\begin{array}{c}\mathrm{Pb} \\
\text { (ppon) }\end{array}$ & $\begin{array}{c}\text { Ti } \\
\text { (ppm) }\end{array}$ & $\begin{array}{c}\text { Cd } \\
\text { (ppm) }\end{array}$ & $\begin{array}{c}B \\
\text { (ppm) }\end{array}$ & $\underset{\text { (ppm) }}{K}$ & $\begin{array}{c}\text { kn } \\
\text { (ppon) }\end{array}$ \\
\hline Mean & 4.903 & 5.101 & 4.919 & 4.846 & 4.969 & 4.975 & 4.853 & 4.912 \\
\hline S.D. & 0.012 & 0.005 & 0.028 & 0.013 & 0.005 & 0.001 & 0.029 & 0.005 \\
\hline \% R.S.D. & 0.237 & 0.104 & $0.5 \pi$ & 0.275 & 0.110 & 0.017 & 0.591 & 0.101 \\
\hline
\end{tabular}

\begin{tabular}{lrrrr} 
& \multicolumn{1}{c}{ sb } & \multicolumn{1}{c}{$v$} & \multicolumn{1}{c}{ Be } & \multicolumn{1}{c}{ Tl } \\
& (ppon) & (ppm) & \multicolumn{1}{c}{ (ppm) } & \multicolumn{1}{c}{ (ppm) } \\
Mean & 4.710 & 4.883 & 5.186 & 5.091 \\
S.D. & 0.037 & 0.011 & 0.011 & 0.026 \\
\% R.S.D. & 0.783 & 0.227 & 0.203 & 0.509
\end{tabular}


Identity 1: CCB Identity 2: Quality Control: $1: 32$ PM July 23, 1997 Task name : OPrIMA Sample Height : 1.0000 solution volume : 1.00 On-Peak Integrations : 3 off-Peak integrations : 1

\begin{tabular}{|c|c|c|c|c|c|c|c|c|}
\hline & $\begin{array}{c}\mathbf{Z r} \\
\text { (ppofi) }\end{array}$ & $\begin{array}{c}\text { sr } \\
\text { (ppon) }\end{array}$ & $\begin{array}{c}B i \\
\text { (pprn) }\end{array}$ & $\begin{array}{c}\mathbf{s i} \\
\text { (ppm) }\end{array}$ & $\underset{\text { (ppm) }}{A !}$ & $\begin{array}{c}\text { Co } \\
\text { (ppm) }\end{array}$ & $\begin{array}{c}\text { Cu } \\
\text { (ppin) }\end{array}$ & $\begin{array}{c}\text { Li } \\
\text { (ppom) }\end{array}$ \\
\hline Heen & -0.002 & 0.000 & -0.007 & -0.002 & 0.000 & 0.010 & 0.001 & 0.000 \\
\hline S.D. & 0.001 & 0.000 & 0.011 & 0.003 & 0.003 & 0.002 & 0.001 & 0.000 \\
\hline \multirow[t]{2}{*}{ \& R.S.D. } & 54.331 & 152.753 & 154.082 & 166.130 & 2024.681 & 19.224 & 57.151 & 44.607 \\
\hline & $\frac{\mathrm{Zn}}{\text { (ppm) }}$ & $\underset{\text { (ppm) }}{N i}$ & $\begin{array}{c}\text { La } \\
\text { (ppm) }\end{array}$ & $\begin{array}{c}\mathrm{Fe} \\
\text { (ppm) }\end{array}$ & $\begin{array}{c}\mathrm{Ca} \\
(\mathrm{ppm})\end{array}$ & $\underset{\text { (ppm) }}{\mathrm{Cr}}$ & $\begin{array}{c}N d \\
\text { (ppons) }\end{array}$ & $\underset{\text { (ppm) }}{U}$ \\
\hline Mean & 0.000 & 0.001 & -0.005 & 0.002 & 0.002 & -0.001 & -0.007 & -0.061 \\
\hline S.D. & 0.000 & 0.001 & 0.001 & 0.001 & 0.000 & 0.002 & 0.009 & 0.046 \\
\hline \multirow[t]{2}{*}{ \% R.S.D. } & 191.626 & 134.128 & 15.245 & 48.917 & 2.749 & 317.479 & 135.189 & 75.136 \\
\hline & $\begin{array}{c}\text { ce } \\
\text { (ppm) }\end{array}$ & $\begin{array}{c}\mathrm{Sm} \\
(\mathrm{ppm})\end{array}$ & $\begin{array}{c}\text { BB } \\
\text { (ppm) }\end{array}$ & $\begin{array}{c}\mathrm{P} \\
\text { (ppm) }\end{array}$ & $\stackrel{s}{\text { (ppm) }}$ & $\begin{array}{c}\text { Mg } \\
\text { (ppm) }\end{array}$ & $\begin{array}{c}\text { As } \\
\text { (Ppm) }\end{array}$ & $\begin{array}{c}\mathrm{Na} \\
\text { (ppm) }\end{array}$ \\
\hline Mean & 0.013 & 0.032 & 0.000 & 0.014 & -0.006 & 0.000 & 0.011 & 0.006 \\
\hline S.D. & 0.012 & 0.010 & 0.000 & 0.010 & 0.003 & 0.000 & 0.002 & 0.004 \\
\hline \multirow[t]{2}{*}{ \% R.S.D. } & 89.107 & 30.585 & 174.356 & 70.598 & 50.704 & 86.604 & 16.334 & 64.956 \\
\hline & $\begin{array}{c}\text { Mo } \\
\text { (ppm) }\end{array}$ & $\begin{array}{c}\mathrm{Ag} \\
\text { (ppm) }\end{array}$ & $\begin{array}{c}\mathrm{Pb} \\
(\mathrm{ppm})\end{array}$ & $\begin{array}{c}\mathrm{Ti} \\
\text { (ppm) }\end{array}$ & $\begin{array}{c}\text { Cd } \\
\text { (ppm) }\end{array}$ & $\begin{array}{c}\text { B } \\
\text { (PPm) }\end{array}$ & $\stackrel{k}{K}$ & $\underset{\text { (ppm) }}{\mathrm{Mn}}$ \\
\hline Mean & -0.002 & 0.005 & 0.032 & -0.001 & 0.003 & -0.004 & -0.033 & 0.000 \\
\hline S.D. & 0.001 & 0.001 & 0.009 & 0.000 & $\quad 0.000$ & 0.001 & 0.031 & 0.000 \\
\hline \% R.S.D. & 70.500 & 17.271 & 28.485 & 72.559 & 17.601 & 16.857 & 94.021 & 163.272 \\
\hline
\end{tabular}

\begin{tabular}{lrrrr} 
& Sb & $v$ & \multicolumn{1}{c}{ Be } & \multicolumn{1}{c}{$T$ Tl } \\
& (ppm) & \multicolumn{1}{c}{ (ppm) } & \multicolumn{1}{c}{ (ppm) } & \multicolumn{1}{c}{ (ppm) } \\
Mean & -0.005 & 0.001 & 0.000 & -0.040 \\
S.D. & 0.022 & 0.001 & 0.000 & 0.033 \\
K R.S.D. & 407.226 & 247.681 & 535.256 & 83.554
\end{tabular}

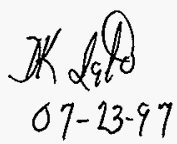


HNF-SD-WM-DP-258, REV. 0

RADIOCHEMICAL ANALYSIS

135.1 
HNF-SD-WM-DP-258, REV. 0

THIS PAGE INTENTIONALLY LEFT BLANK

$135 \cdot 2$ 


\section{LABCORE Completed RadChem Report for Worklist\#: 19397}

Analyst: scl

Instrument: $\mathrm{AB} 14$

Book\#

Method:

Rev/Mod

Worklist Comment: BY-101 ALPHA, DETERMINE SS USING LUDLUM. RCJ

\begin{tabular}{|c|c|c|c|c|c|c|c|c|}
\hline Seq Type & \multicolumn{2}{|c|}{ Sample\# R A } & \multicolumn{2}{|r|}{ Test } & Matrix & Actual & \multicolumn{2}{|c|}{ Found DL or Yield Unit } \\
\hline 1 SIt & & o & 6 AT 1401 & 040100 & Toun & $2008-04$ & $103 \mathrm{~s}$ & xhrovoxy \\
\hline $1 \mathrm{STD}$ & & 0 & OAPEAOI & ALPEAO1B & IIOUID & 1.00 & $3.45 z+00$ & \& Ct. Erro \\
\hline $2 \mathrm{sin} / \mathrm{x}$ & & o & 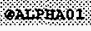 & 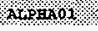 & wovto & 11 & $87 \times 1127$ & $101 / m$ \\
\hline 2 BLNR & & 0 & OAIPEAOI & ALPEAO1B & IIQUID & 1.00 & $5.00 \mathrm{E}+02$ & 500,000 \\
\hline 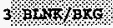 & & 0 & $04=2901$ & $442+201$ & 4010 & $1000+00$ & $1722 \mathrm{~s} \% \mathrm{I}$ & $84 / 8 K S$ \\
\hline 4 SAMPLB & 5979001365 & 0 & GALPEAO1 & ALPEAO1 & LIQUID & X/A $<$ & $7.81 E-07$ & $187.0 e-008$ \\
\hline 4 sppt & 8974001365 & 0 & QA+PHAO1 & $A=p=018$ & LIQUID & $\mathrm{N} / \mathrm{A}, \mathrm{W}$ & $5000+02$ & $140 / x+\alpha=$ \\
\hline 5 DUP & $597 T 001365$ & 0 & OALPHAOI & ALPEAOI & IIOUID & $<7.81 \mathrm{E}-7$ & $<7.81 \mathrm{E}-7$ & RPD \\
\hline 5 pup & 8971001365 & 0 & 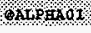 & $\alpha+8=0,8$ & 100010 & 100 & $5002+02$ & 500000 \\
\hline
\end{tabular}

\section{Final page for worklist\# 19397}

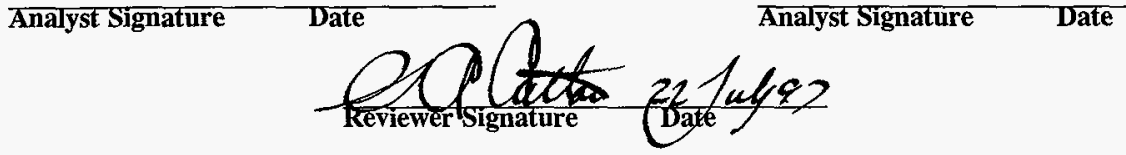

Units shown for $Q C \overline{(B L K / B K G)}$ may not reflect the actual units. 


\section{LABCORE Data Entry Template for Worklist\# 19397}

Analyst: $\quad 3.2$ Instrument: $\mathrm{AB} 00 \_14$ Book\# 79856

Method: LA-508-101 Rev/Mod G-0

Worklist Comment: BY-101 ALPHA, DETERMINE SS USING LUDLUM, RCJ

\begin{tabular}{|c|c|c|c|c|c|c|}
\hline$\overline{\text { s Type }}$ & Sample\# & $\overline{R A}$ & Test & Matxix & Group\# & Project \\
\hline I STD & & & QALPHAOI & IIQUID & & \\
\hline 2 BLNK & & & @ALPHAO1 & LIQUID & & \\
\hline 3 BLNK/BKG & & & @ALPHAOI & LIQUID & & \\
\hline 4 SAMPLE & $\begin{array}{l}\text { S97T001365 } \\
\text { Analytes Reque }\end{array}$ & $\begin{array}{l}0 \\
\text { ested: }\end{array}$ & $\begin{array}{r}\text { QALPHAO 1 } \\
: \text { ALPHAO1 }\end{array}$ & $\begin{array}{l}\text { LIQUID } \\
\text { ALPHA01E }\end{array}$ & 97000397 & $B Y-101$ \\
\hline 5 DUP & S97T001365 & 0 & QALPHAO 1 & LIQUID & & \\
\hline
\end{tabular}

$\frac{L_{\text {Sud }}}{7-21-97}$

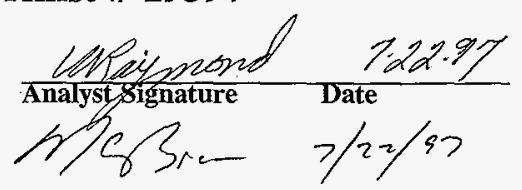

Data Entry Comments:

$\bar{S}=$ Worklist Slot Number, $R=$ Replicate Number, $A=$ Aliquot Code. 
WORKBOOK PAGE: STD1

AT : LA-508-101 (E-1)

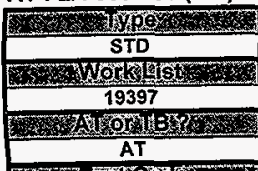

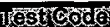

(QALPHA01

$\frac{\text { MFGX }}{\text { LQQUD }}$

A-508-113 (B-0) STANDARD

DETECTOR NUMBER

DISH SIZE $\quad(1,2$, or 5$)$

GROSS COUNTS

COUNT TIME in MINUTES

BACKGROUND in cpm

SAMPLE SIZE in $\mathrm{mL}$

DILUTION FACTOR

STANDARD BOOK NUMBER

EFFICIENCY FACTOR

Lc, Rmax, or Rs,(SAMPLE RATE) as APPROPRIATE

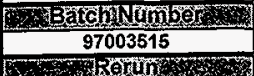

Standard Value in $\mu \mathrm{Ci} / \mathrm{mL}$

Concentration in $\mu \mathrm{C} / \mathrm{L}=1.85 \mathrm{E}-01$

Rerun 2 Replicate Concentration in $\mu \mathrm{Ci} / \mathrm{L}$

AVERAGE CONCENTRATION in $\mu \mathrm{CI} / \mathrm{L}=1.8270 \mathrm{E}-01$

\section{7\%}

N/A

Rs (Sample Count Rate) $=($ TC / CT $)$ - BKG

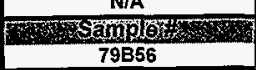

ALPHA TOTAL $\mu \mathrm{CI} / \mathrm{L}=\mathrm{Rs}$ * $1000 \mathrm{~mL} / \mathrm{L}^{*} \mathrm{DF} /(\mathrm{EFF} * \mathrm{SS} * 2220000 \mathrm{dpm} / \mathrm{\mu Ci})$

ALPHA TOTAL $\mu \mathrm{CI} / \mathrm{mL}=$ ALPHA TOTAL $\mu \mathrm{CI} / \mathrm{L} / 1000 \mathrm{mLL}$

nstwing

WB27807

Relative Counting Error = [I(The Square Root of TC + BKG *CT) / (TC $-B K G * C T) \mid] * 1.96$ * 100

Detection Levels and Less Than Values are determined from Procedure LA-508-002.

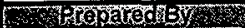

VAR

W.

\begin{tabular}{|c|c|}
\hline \multirow{2}{*}{\multicolumn{2}{|c|}{$\begin{array}{c}\text { SLF } \\
\text { Trom }\end{array}$}} \\
\hline & \\
\hline SCL & \\
\hline 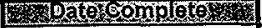 & \\
\hline $07 / 22 / 97$ & \\
\hline 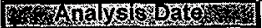 & \\
\hline $07 / 21 / 97$ & \\
\hline HAnalysis & \\
\hline $10: 20 \mathrm{AM}$ & \\
\hline 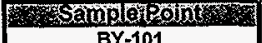 & \\
\hline BY-101 & \\
\hline
\end{tabular}

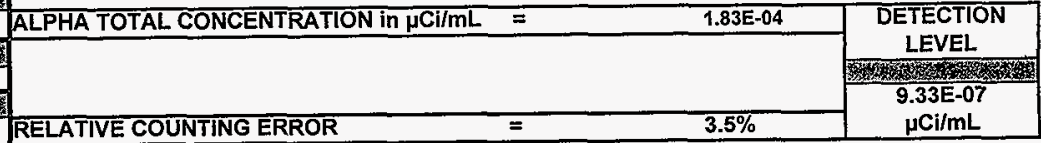

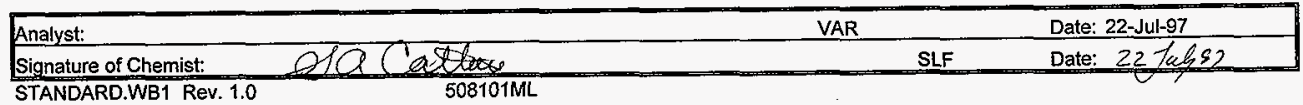

\section{8}


HNF-SD-WM-DP-258, REV. 0

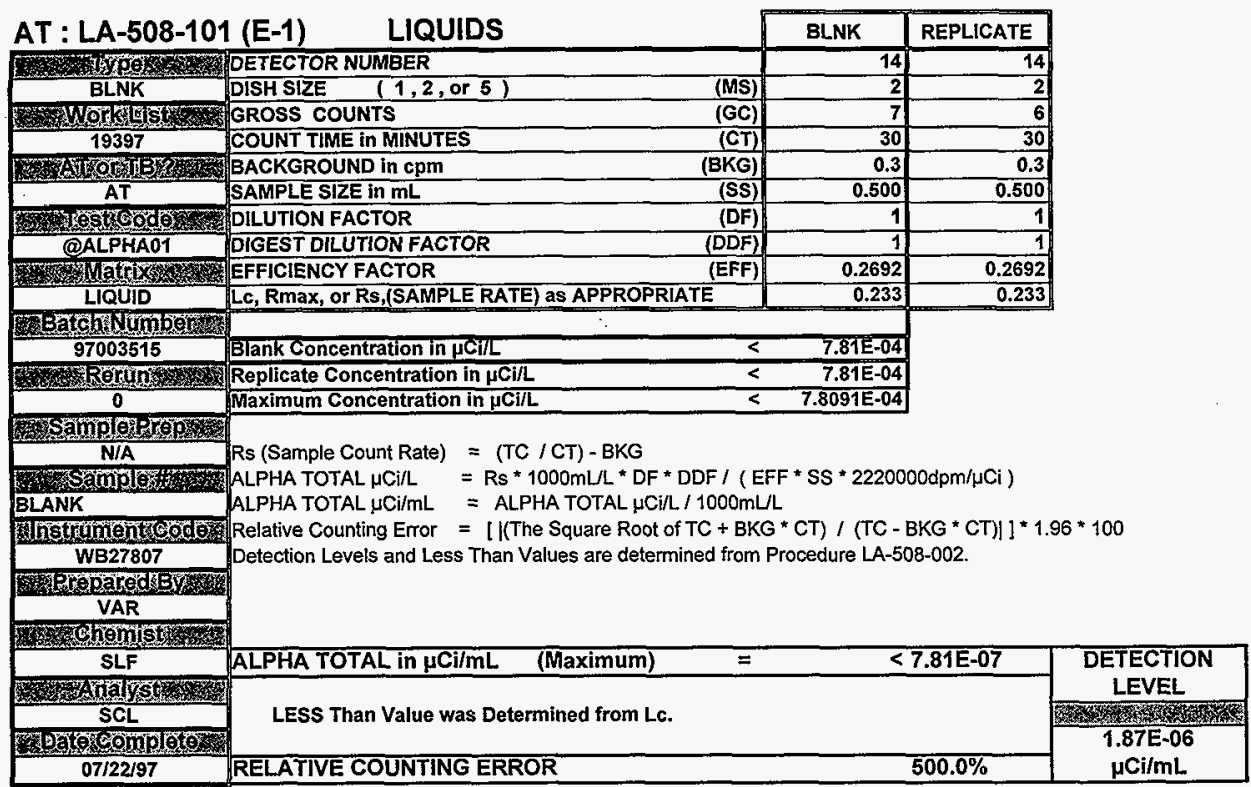

Yronalvs $07 / 21 / 97$

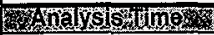
10:20 AM

r samplom Solnt BY-101

\begin{tabular}{|c|c|c|}
\hline Analyst: & SCL. & Date: 22-Jul-97 \\
\hline Signature of Chemist: & SLF & Date: $22-144 ? 2$ \\
\hline
\end{tabular}


WORKBOOK PAGE: SAM4

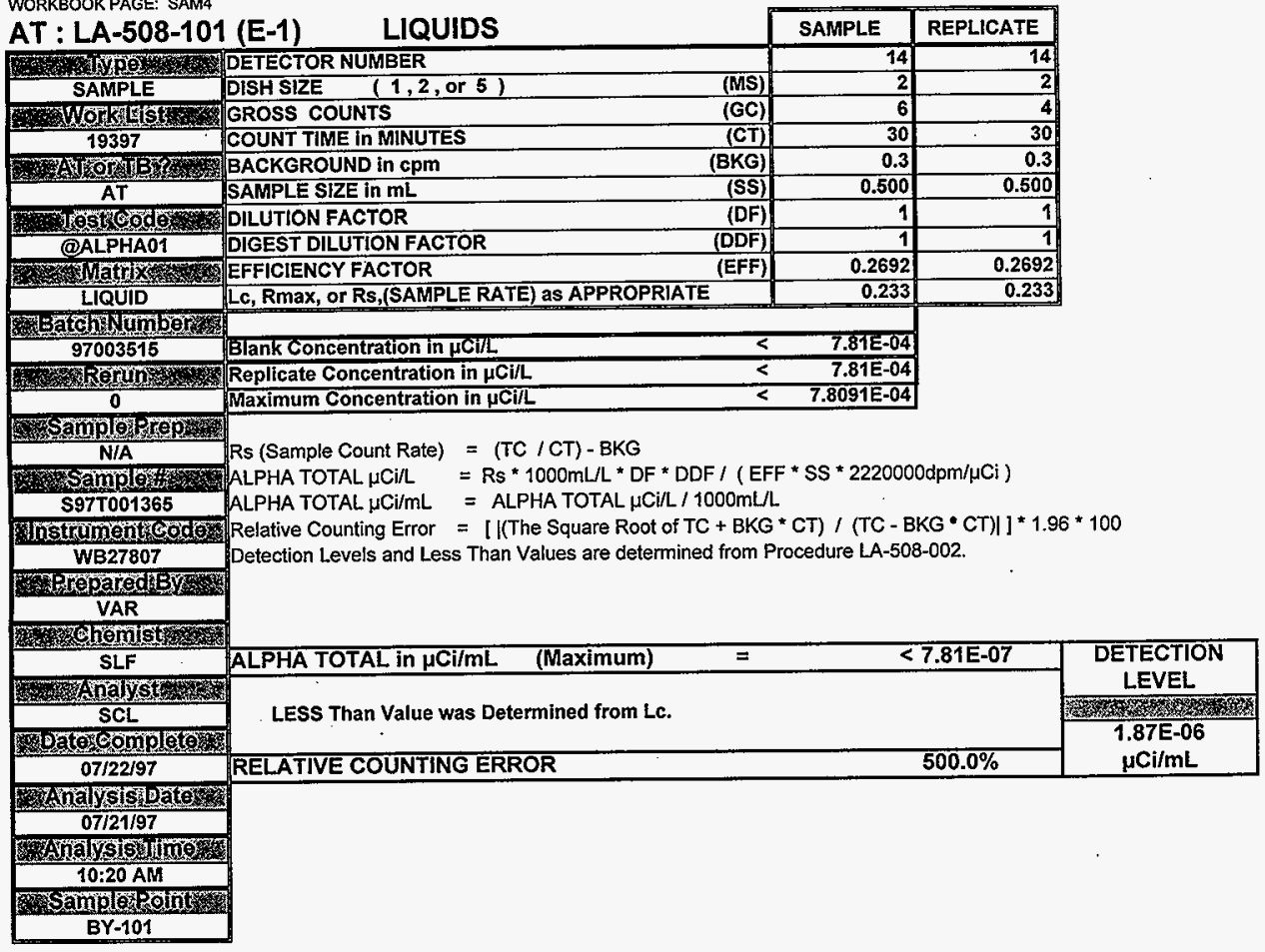

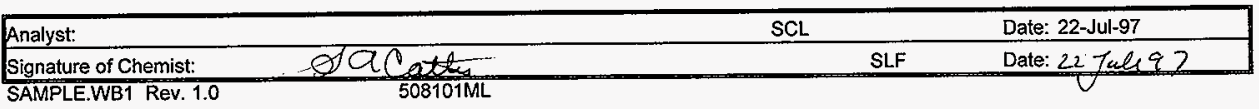


WORKBOOK PAGE: DUPS

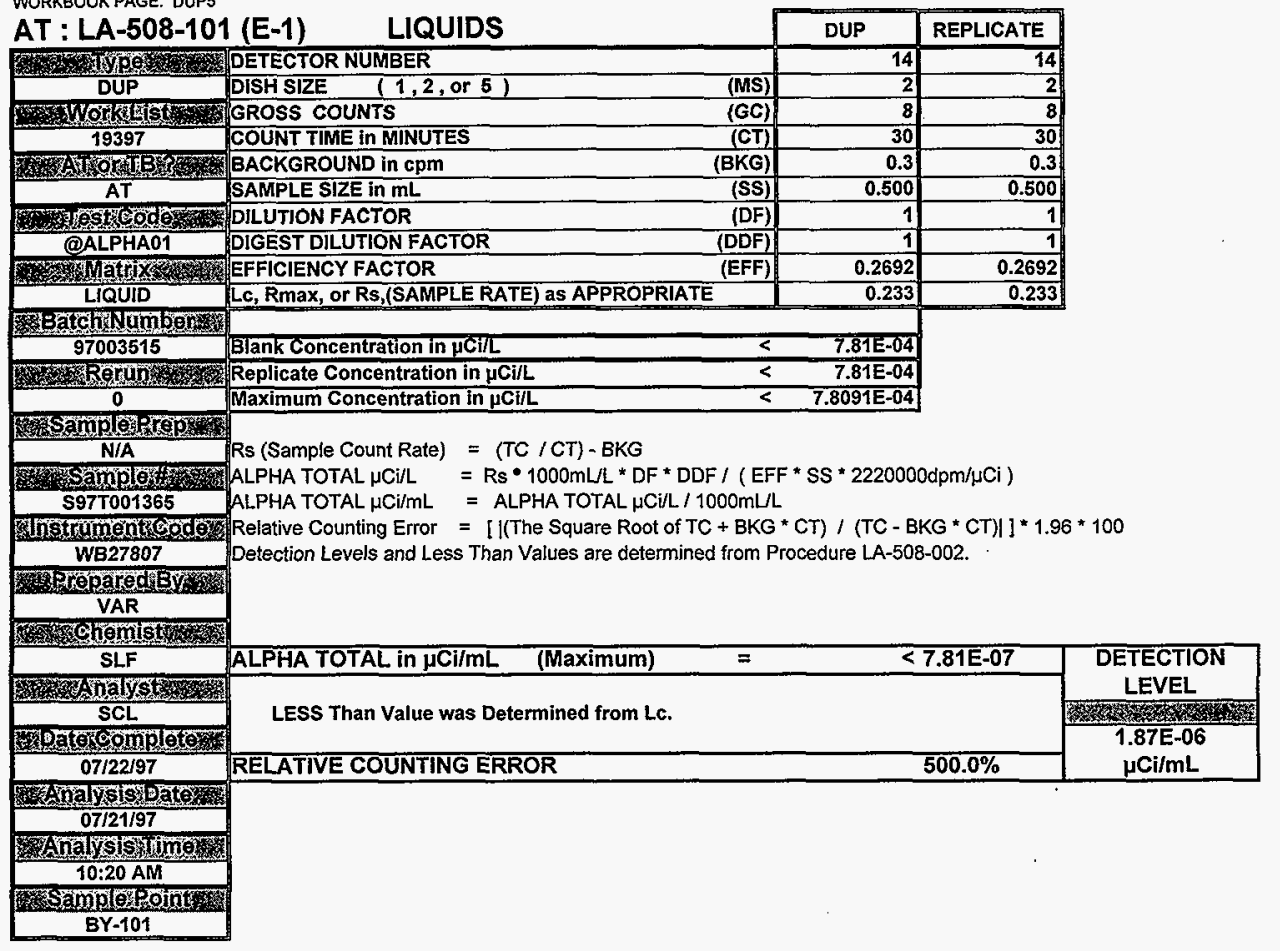

\begin{tabular}{|c|c|c|}
\hline Analyst: & $S C L$ & Date: 22-Jul-97 \\
\hline Signature of Chemist: & SLF & Date: $22,4,592$ \\
\hline
\end{tabular}

\section{1}




\section{LABCORE Completed RadChem Report for Worklist\#: 19405}

Analyst: smf

Instrument: $A B 15$

Book\#

Method:

Rev/Mod

Worklist Comment: BY101, @ALPHA01, S.S: By Ludlum. STD: 1.0mL, SPK: 0.1mL skm

\begin{tabular}{llllll}
\hline Seq Type & Sample\# R A & Test & Matrix Actual & Found DL or Yield Unit
\end{tabular}

\begin{tabular}{|c|c|c|c|c|c|c|c|c|c|}
\hline 1 sto & & 0 & 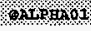 & XIp & solno & $2000 \mathrm{~B}-01$ & $1911 \%$ & 35500 & $360-0-10 \mathrm{~V}$ \\
\hline 1 STD & & 0 & GALPEA01 & ALPHA01E & SOLID & 1.00 & $3.36 B+00$ & 3.360 & \&t. Bxro \\
\hline $2 / \mathrm{BHT} / \mathrm{pRS}$ & & $\%$ & $\angle A-12401$ & $x_{1} x_{212} 01$ & soldo & 12 & $41,69 \mathrm{~B}$ & & 20 \\
\hline 2 BLNK-PRBP & & o & EALPHA01 & ALPHAOIE & SOLID & 1.00 & $5.008+02$ & 500.000 & $\mathrm{uCl} / \mathrm{g}$ \\
\hline 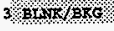 & & o. & Q & 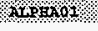 & solin & $1002+00$ & $12432+00$ & $1 / 40$ & 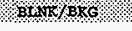 \\
\hline 4 SAMSLE & S97T001358 & $0 F$ & OAIPEAO1 & ALPHA01 & SOLID & $W / A<$ & $3.09 \mathrm{E}-03$ & $268.00-005$ & $\mathrm{uCl} / \mathrm{g}$ \\
\hline 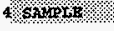 & 5971001358 & $0 \%$ & 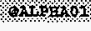 & 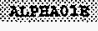 & 8041 & 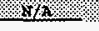 & 4500002 & 0000000 & $6 \mathrm{ct}-\mathrm{s}$ \\
\hline 5 DOP & S97T001358 & OF & OALPHA01 & ALPEA01 & SOLID & $<3.09 \mathrm{E}-3$ & $<1.69 \mathrm{~g}-3$ & & RPD \\
\hline 5000 & 5570001358 & 0 & $6418 A_{01}$ & 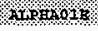 & solnt & $1 \% 00$ & $2=558+02$ & 255000 & 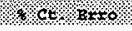 \\
\hline $6 \mathrm{SPR}$ & s97T001358 & 08 & GALPHA01 & ALpHA01 & SOLID & $2.63 E-02$ & $1.85 \mathrm{E}-02$ & 70.342 & \& Recovery \\
\hline $7 \%$ supto & $597+00060$ & $0 \%$ & $64=1901$ & 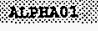 & $5 \circ 1+0$ & 878 & 224503 & $262=0-1005$ & $\alpha c t s$ \\
\hline 7 sAMPLE & S97T001363 & $0 F$ & OALPBA01 & ALPHA018 & SOLID & N/A & $1.06 \mathrm{~B}+02$ & $0.0 e+000$ & \& Ct. Brror \\
\hline 8 out & 501700130 & 0.1 & $0 \times 1 \times 1+\alpha 01$ & 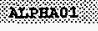 & 50110 & $2 / 48-3$ & 1,248 & 4464 & 80. \\
\hline 8 DUP & S97T001363 & $0 F$ & OALPBA01 & ALPHAOLB & SOLID & 1.00 & $1.21 E+02$ & 121.000 & * ct. Brro \\
\hline $9.5 \mathrm{p}$ & 5971001360 & 01 & 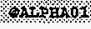 & X+101201 & $501+1$ & 2.65002 & 102100 & $65 \times 79$ & 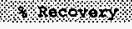 \\
\hline
\end{tabular}

\section{Final page for worklist\# 19405}

$\overline{\text { Analyst Signature Date }}$ Analyst Signature Date

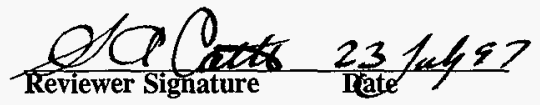

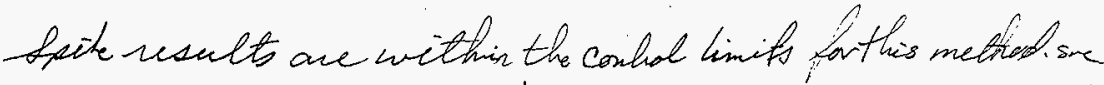
High RPD caused by eounting uncertainties greater than $100 \%$. Results will be accepted. sne 


\section{LABCORE Data Entry Template for Worklist\# 19405}

\section{Analyst: $\quad$ BMF Instrument: $\mathrm{AB} 00$ is Book\# 79856}

Method: LA-508-101 Rev/Mod 6-O

Worklist Comment: BY101, @ALPHA01, S.S. By Ludlum. STD: 1.0mL, SPK: 0.1mL skm

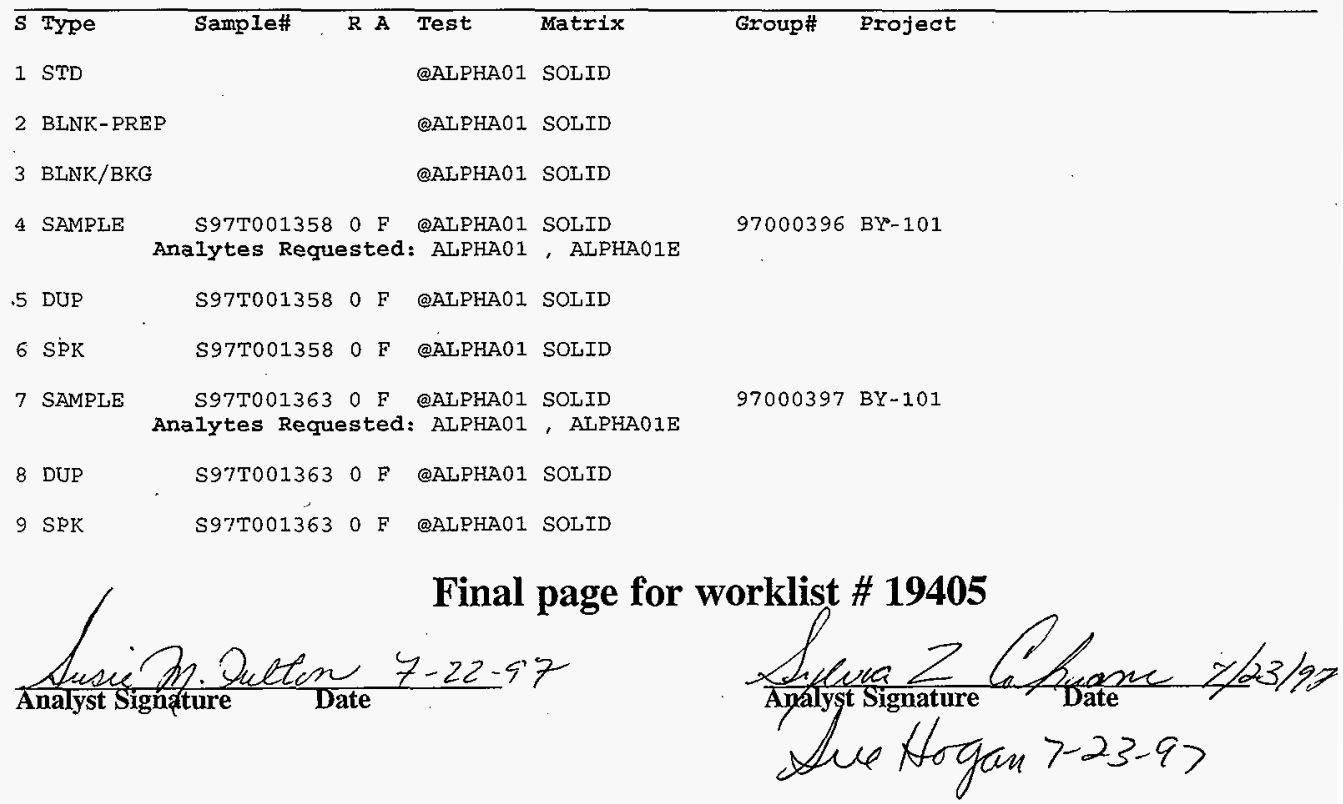

Data Entry Comments:

$\bar{S}=$ Worklist Slot Number, $R=$ Replicate Number, $A=$ Aliquot Code. 
WORKBOOK PAGE: STD1

AT : LA-508-101 (E-1)

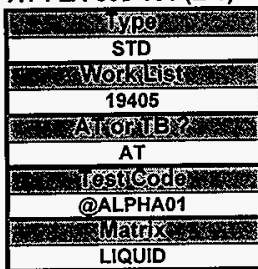

LA-508-113 (B-0) STANDARD

DETECTOR NUMBER

DISH SIZE

GROSS COUNTS

COUNT TIME in MINUTES

BACKGROUND in cpm

SAMPLE SIZE in $\mathrm{mL}$

DILUTION FACTOR

STANDARD BOOK NUMBER

EFFICIENCY FACTOR

Lc, Rmax, or Rs,(SAMPLE RATE) as APPROPRIATE

Bachinutwhes 97003525

Standard Value in $\mu \mathrm{Ci} / \mathrm{mL}$

Concentration in $\mu \mathrm{CF} / \mathrm{L}$.

or 5

1

Replicate Concentration in $\mu \mathrm{Ci} / \mathrm{L}$

AVERAGE CONCENTRATION in $\mu \mathrm{CIL}=1.9134 \mathrm{~L}-01$

\begin{tabular}{r|r|r|}
\cline { 2 - 3 } \multicolumn{1}{c|}{} & STANDARD & REPLICATE \\
\hline (MS) & 15 & 15 \\
\hline (GC) & 2 & 2 \\
\hline (CT) & 3487 & 3401 \\
\hline BKG) & 30 & 30 \\
\hline
\end{tabular}

(DS)

(Std BN)

79B56

000

.000

\begin{tabular}{|c|}
\hline Dorun \\
\hline 0 \\
\hline N/A
\end{tabular}
STup WL19405

Rs (Sample Count Rate) $=(T C / C T)-B K G$

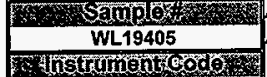

ALPHA TOTAL $\mu \mathrm{Ci} / \mathrm{L}=$ Rs $* 1000 \mathrm{~mL} / \mathrm{L} * \mathrm{DF} /(\mathrm{EFF} * \mathrm{SS}$
ALPHA TOTAL $\mu \mathrm{CI} / \mathrm{mL}=$ ALPHA TOTAL $\mu \mathrm{Ci} / \mathrm{L} / 1000 \mathrm{mLL}$

$=R S * 1000 \mathrm{~mL} / \mathrm{L} * \mathrm{DF} /(\mathrm{EFF} * \mathrm{SS} * 2220000 \mathrm{dpm} / \mu \mathrm{Ci})$ WB26872

Relative Counting Error $=\left[\left(\mid\left(T h e \text { Square Root of TC }+B K B^{*} \mathrm{CT}\right) /(T \mathrm{C}-\mathrm{BKG} * \mathrm{CT})\right]\right]^{*} 1.96 * 100$ Detection Levels and Less Than Values are determined from Procedure LA-508-002.

\%

$$
\text { SZC }
$$

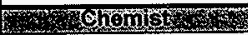
SLF ALPHA TOTAL CONCENTRATION in $\mu \mathrm{Ci} / \mathrm{mL}=$

$=1.91 \mathrm{E}-04$

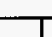

$\frac{\text { T.Malyg }}{\text { SMF }}$

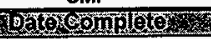
07/23/97

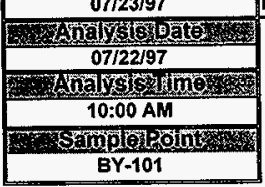

Analyst:

Signature of Chemist:

STANDARD.WB1 Rev. 1.0
SZC

SAt Sathor

$508101 \mathrm{ML}$
Date: 23-Jul-97 SLF

Date: $232 x 42$ 
WORKBOOK PAGE: BLANK2

\begin{tabular}{|c|c|c|c|c|}
\hline & & \\
\hline & & BLNK-PREP & REPLICATE & \\
\hline 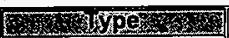 & DETECTOR NUMBER & 15 & 15 & \\
\hline BLNK-PREP & DISH SIZE $\quad(1,2$, or 5$)$ & 2 & 2 & \\
\hline WWNOLISISI & GROSS COUNTS & 2 & 4. & \\
\hline 19405 & COUNT TIME In MINUTES & 30 & 30 & \\
\hline 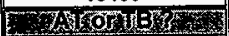 & BACKGROUND in cpm & 0.07 & 0.07 & \\
\hline AT & SAMPLE SIZE in $\mathrm{mL}$ & 0.100 & 0.100 & \\
\hline 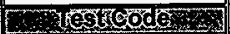 & DILUTION FACTOR & 1 & 1 & \\
\hline (QALPHA01 & DIGEST GRAMS of SOLIDS / L & 1.964 & 1.964 & . \\
\hline Nathis & EFFICIENCY FACTOR & 0.2701 & 0.2701 & \\
\hline SOLID & Lc, Rmax, or Rs,(SAMPLE RATE) as APPROPRIATE & 0.113 & 0.199 & \\
\hline Batow THOST & & & & \\
\hline 97003525 & Bank Concentration in $\mu \mathrm{CI} / \mathrm{g}$ & 9.57E-04 & & \\
\hline 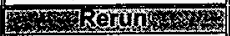 & Replicate Concentration in $\mu \mathrm{Ci} / \mathrm{g}$ & $1.69 \mathrm{E}-03$ & & \\
\hline 0 & Maximum Concentration in $\mu \mathrm{Ci} / \mathrm{g}$ & $1.6913 E-03$ & & \\
\hline Hotmogerep & & & & \\
\hline N/A & Rs (Sample Count Rate) $=(\mathrm{TC} / \mathrm{CT})-\mathrm{BKG}$ & & & \\
\hline \% samplex & ALPHA TOTAL $\mu \mathrm{Ci} / \mathrm{g}=\mathrm{Rs} * 1000 \mathrm{~mL} / \mathrm{L} * \mathrm{DF} /(\mathrm{EFF} * \mathrm{SS}$ & $S^{*} \mathrm{Dg} / \mathrm{L} * 22200$ & $00 \mathrm{dpm} / \mu \mathrm{Ci})$ & \\
\hline WL 19405 & & & & \\
\hline Whstromose & Relative Counting Error $=[1($ The Square Root of $\mathrm{TC}+\mathrm{BKC}$ & $* \mathrm{CT}) /(\mathrm{TC}-\mathrm{B}$ & $3 K G$ * $C T) \mid]$ * 1.96 & $5 * 100$ \\
\hline WB26872 & Detection Levels and Less Than Values are determined from & Procedure LA-50 & $8-002$ & \\
\hline 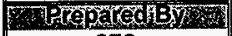 & & & & \\
\hline SZC & & & & \\
\hline chom & & & & \\
\hline SLF & ALPHA TOTAL in $\mu \mathrm{Ci} / g$ & $\leq$ & 1.69E-03 & DETECTION \\
\hline 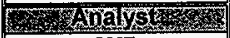 & & & & LEVEL. \\
\hline SMF & LESS Than Value was Determined from Lc. & & & 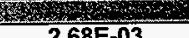 \\
\hline 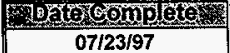 & RELATIVE COUNTING ERROR & & $500.0 \%$ & $\begin{array}{c}\text { 2.68E-U3 } \\
\mu \mathrm{Ci} / \mathrm{g}\end{array}$ \\
\hline
\end{tabular}

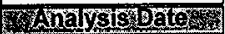
$07 / 22 / 97$

7hatsis stowe 10:00 AM

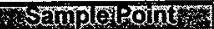

BY-101

\begin{tabular}{|c|c|c|}
\hline Analyst: & SMF & Date: 23-Jul-97 \\
\hline Signature of Chemist: & & Date: 23 tal \&2 \\
\hline
\end{tabular}


WORKBOOKPAGE: SAM4

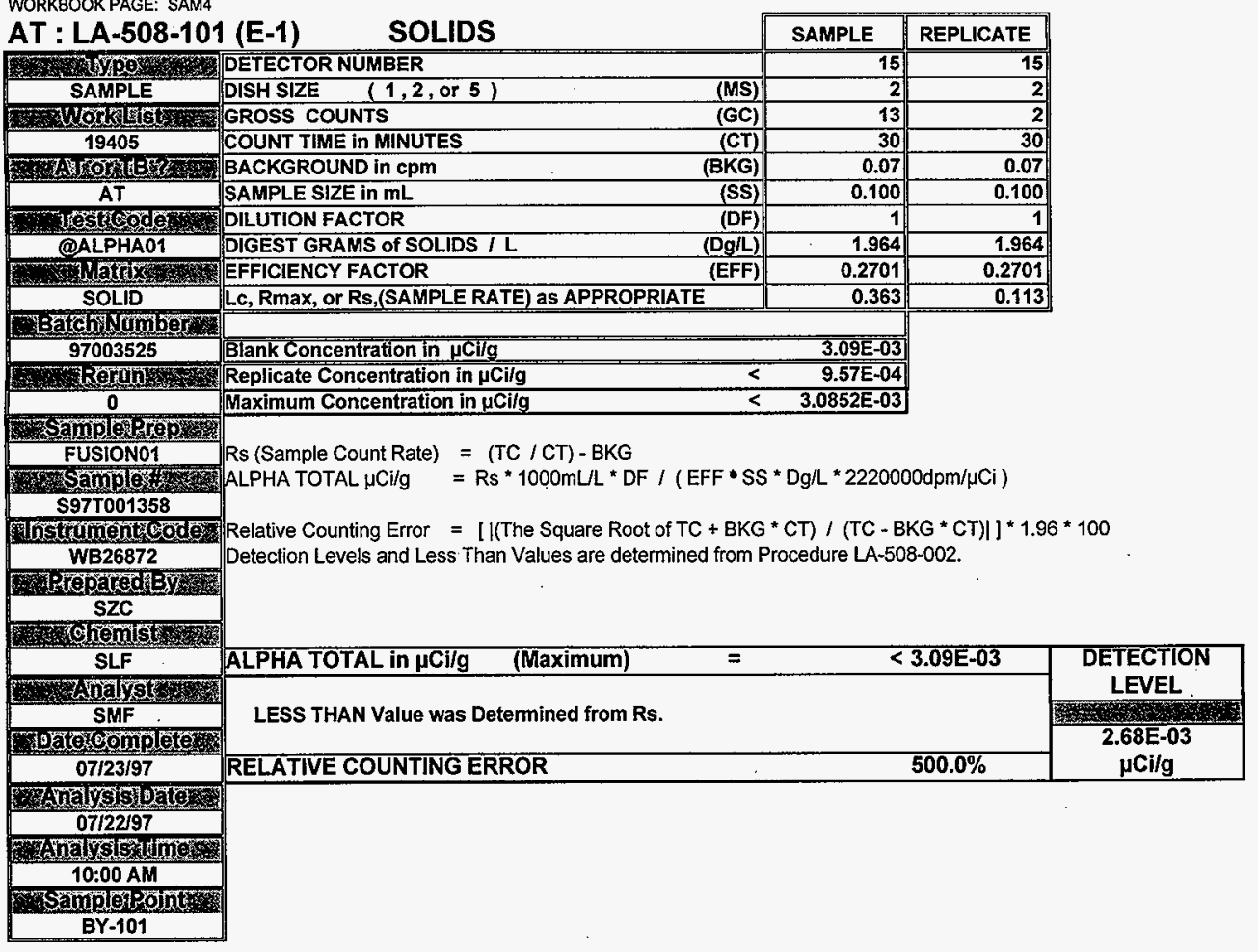

\begin{tabular}{|c|c|c|c|}
\hline Analyst: & & SMF & Date: $23-J u l-97$ \\
\hline Signature of Chemist: & $\theta$ COation & SLF & Date: 2312492 \\
\hline
\end{tabular}


HNF-SD-WM-DP-258, REV. 0

WORKBOOK PAGE: DUP5

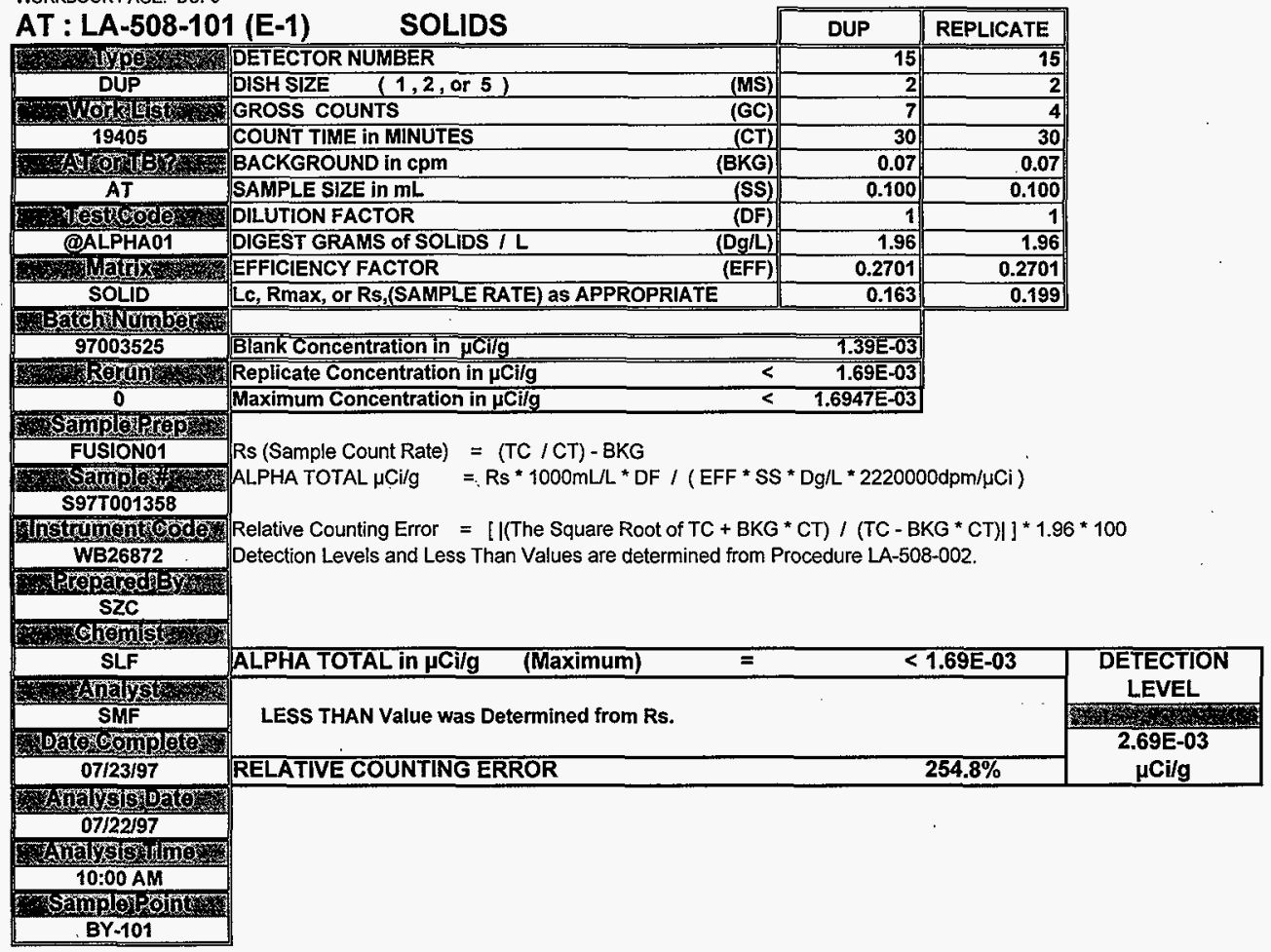

\begin{tabular}{|c|c|c|c|}
\hline Analyst: & & SMF & Date: $23-J u l-97$ \\
\hline Signature of Chemist: & 870 atk & SLF & Date: 2314492 \\
\hline
\end{tabular}


WORKBOOK PAGE: SPK6

\begin{tabular}{|c|c|c|c|}
\hline \multirow{2}{*}{\multicolumn{2}{|c|}{ AT : LA-508-101 (E-1) LA-508-113(B-0) SPIKED SAMPLE }} & \multirow{2}{*}{\multicolumn{2}{|c|}{ SDIKE }} \\
\hline & & & \\
\hline 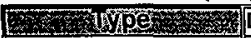 & DETECTOR NUMBER & 15 & 15 \\
\hline SPK & DISH SIZE $\quad 1,2$, or 5 & 2 & 2 \\
\hline 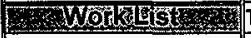 & TOTAL COUNTS & 32986 & 33681 \\
\hline 19405 & COUNT TIME in MINUTES & 30 & 30 \\
\hline 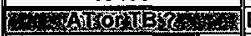 & BACKGROUND in cpm & 0.07 & 0.07 \\
\hline AT & SAMPLE VOLUME in $\mathrm{mL}$ & 0.100 & 0.100 \\
\hline LesEOOde & SAMPLE DILUTION FACTOR (Spiked Vial) & 1 & 1 \\
\hline @ALPHAO1 & DIGEST GRAMS of SOLIDS / $L$ & 1.964 & 1.964 \\
\hline Wath $\mathrm{X}$, & SPIKE VOLUME in $\mathrm{mL}$ & 0.100 & 0.100 \\
\hline SOLID & SPIKE DILUTION FACTOR & 1 & 1 \\
\hline Batch Nombe & SPIKE BOOK NUMBER & 131B43 & 131B43 \\
\hline 97003525 & SPIKE VALUE in $\mu \mathrm{Ci} / \mathrm{mL}$ & $2.6286 \mathrm{E}-02$ & $2.6286 \mathrm{E}-02$ \\
\hline X Rerun & INSTRUMENT EFFICIENCY FACTOR & 0.2701 & 0.2701 \\
\hline $\mathbf{0}$ & SAMPLE + SPIKE $\mu$ Cilg & $9.34 \mathrm{E}+00$ & $9.53 E+00$ \\
\hline 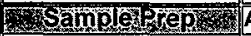 & AVERAGE or MAXIMUM $\mu \mathrm{Ci} / \mathrm{g}$ in SAMPLE & 3.0852E-03 & \\
\hline FUSIONO1 & & & \\
\hline 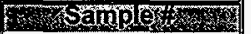 & & & \\
\hline S97T001358 & Rs (Sample Count Rate) $=(\mathrm{TC} / \mathrm{CT})-\mathrm{BKG}$ & & \\
\hline 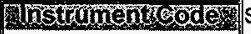 & $=R s * 1000 \mathrm{~mL} / \mathrm{R}^{*} \mathrm{DF} /(\mathrm{EFF} * \mathrm{SS}$ & ${ }^{*} \mathrm{Dg} / \mathrm{L}$ *2220000 & $\mathrm{dpm} / \mu \mathrm{C} i$ ) \\
\hline WB26872 & QC ACTUAL = SVal & & \\
\hline Lereareogsy & QC FOUND $=\left(\left((\mathrm{S}+\mathrm{S} \mu \mathrm{Ci} / \mathrm{g}-\mathrm{SAMPLE} \mu \mathrm{Ci} / \mathrm{g}) *\left(\left(\mathrm{SDF} /(\mathrm{SVO})^{*} 1 \mathrm{C}\right.\right.\right.\right.$ & Doo))/(DF/SS/Dg/ & (L)))) \\
\hline SzC & PERCENT SPIKE RECOVERY = (QC FOUND / QC ACTUAL ) * & & \\
\hline Whomis & & & \\
\hline SLF & & & \\
\hline 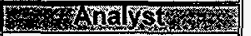 & & & \\
\hline SMF & & & \\
\hline 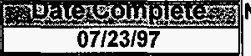 & $\begin{array}{l}\text { Original Sample result was a LESS THAN value. } \\
\text { spiked value for } Q C \text { found calculation. }\end{array}$ & Zero (0) was sut & btracted from the \\
\hline 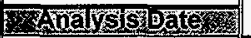 & & & \\
\hline $07 / 22 / 97$ & QC ACTUAL & 2.63E-02 & \\
\hline 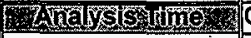 & QC FOUND & 1.85E-02 & \\
\hline 10:00 AM & AVG. PERCENT SPIKE RECOVERY & $70.5 \%$ & \\
\hline 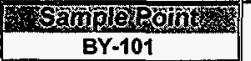 & & & \\
\hline Analyst: & & SZC & Date: 23-JuJ-97 \\
\hline Signature of Chemist: & Siscato & SLF & Date: 232,22 \\
\hline
\end{tabular}




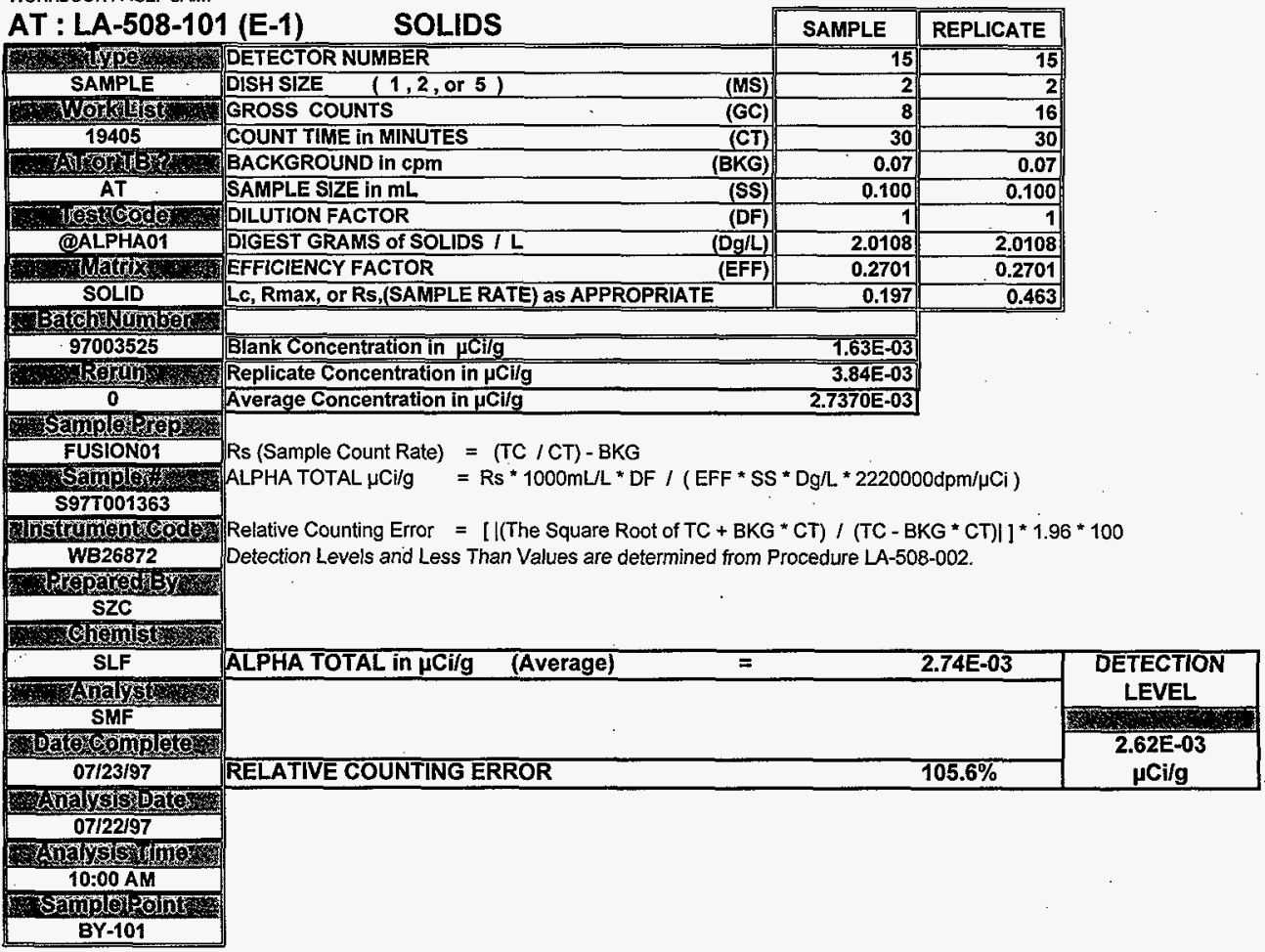

\begin{tabular}{|c|c|c|c|}
\hline Analyst: & & SMF & Date: $23-J u l-97$ \\
\hline Signature of Chemist: & Sidcats & SLF & Date: 2312482 \\
\hline
\end{tabular}


HNF-SD-WM-DP-258, REV. 0

WORKBOOK PAGE: DUPB

\begin{tabular}{|c|c|c|c|c|}
\hline AT : LA-508-101 & SOLIDS & DUP & REPLICATE & \\
\hline 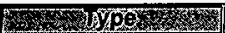 & DETECTOR NUMBER & 15 & 15 & \\
\hline DUP & DISH SIZE $\quad(1,2$, or 5$)$ & 2 & 2 & \\
\hline Workigers & GROSS COUNTS & 7 & 10 & \\
\hline 19405 & COUNT TIME in MINUTES & 30 & 30 & \\
\hline 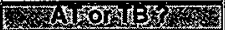 & BACKGROUND in cpm & 0.07 & 0.07 & \\
\hline AT & SAMPLE SIZE in $\mathrm{mL}$ & 0.100 & 0.100 & \\
\hline Wuestecos & DILUTION FACTOR & 1 & 1 & \\
\hline @ALPHA01 & DIGEST GRAMS of SOLIDS / L & 2.0424 & 2.0424 & \\
\hline 2\% watris, & EFFICIENCY FACTOR & 0.2701 & 0.2701 & \\
\hline SOLID & Lc, Rmax, or Rs,(SAMPLE RATE) as APPROPRIATE & 0.163 & 0.263 & \\
\hline Batch Number & & & & \\
\hline 97003525 & Blank Concentration in $\mu \mathrm{Ci} / \mathrm{g}$ & $1.33 \mathrm{E}-03$ & & \\
\hline H x & Replicate Concentration in $\mu \mathrm{Ci} / \mathrm{g}$ & 2.15E-03 & & \\
\hline 0 & Average Concentration in $\mu \mathrm{Cl} / \mathrm{g}$ & 1.7420E-03 & & \\
\hline 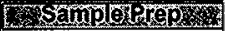 & & & & \\
\hline FUSION01 & Rs (Sample Count Rate) $=(\mathrm{TC} / \mathrm{CT})-\mathrm{BKG}$ & & & \\
\hline 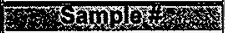 & $=\mathrm{Rs}^{*} 1000 \mathrm{~mL} /{ }^{*} \mathrm{DF} /(\mathrm{EFF} * \mathrm{SS}$ & $\mathrm{Dg} / \mathrm{L}$ *222000 & $\mathrm{DOdpm} / \mu \mathrm{Ci})$ & \\
\hline S97T001363 & & & & \\
\hline 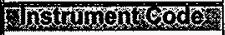 & Relative Counting Error $=[\mid(T$ The Square Root of $\mathrm{TC}+\mathrm{BKG}$ & $\mathrm{CT}) /(\mathrm{TC}-\mathrm{BH}$ & $\left.\left.\mathrm{KG}^{*} \mathrm{CT}\right) \mid\right]^{* 1.96}$ & * 100 \\
\hline WB26872 & Detection Levels and Less Than Values are determined from P & ocedure LA-508 & 8-002. & \\
\hline Wregaradiy & & & & \\
\hline SZC & & & & \\
\hline 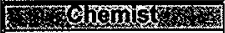 & & & & \\
\hline SLF & ALPHA TOTAL in $\mu \mathrm{Ci} / \mathrm{g}$ & & $1.74 \mathrm{E}-03$ & DETECTION \\
\hline Whalys & & & & LEVEL \\
\hline SMF & & & & 4 \\
\hline Bargeompotor & & & & $2.58 E-03$ \\
\hline $07 / 23 / 97$ & RELATIVE COUNTING ERROR & & $120.7 \%$ & $\mu \mathrm{Ci} / \mathrm{g}$ \\
\hline Wuarsis & & & & \\
\hline $07 / 22 / 97$ & & & & \\
\hline 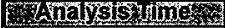 & & & & \\
\hline 10:00 AM & & & & \\
\hline 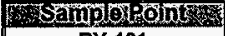 & & & & \\
\hline BY-101 & & & & \\
\hline
\end{tabular}

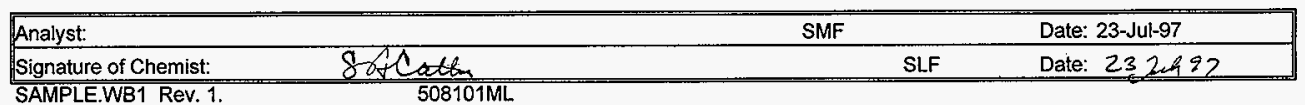


WORKBOOK PAGE: SPK9

\begin{tabular}{|c|c|c|c|}
\hline \multicolumn{2}{|l|}{ AT : LA-508-101 (E-1) } & SPIKE & REPLICATE \\
\hline Wunpe & DETECTOR NUMBER & 15 & 15 \\
\hline SPK. & DISH SIZE & 2 & 2 \\
\hline WOMgla & TOTAL COUNTS & 30952 & 31230 \\
\hline 19405 & COUNT TIME IN MINUTES & 30 & 30 \\
\hline 3) & BACKGROUND in cpm & 0.07 & 0.07 \\
\hline AT & SAMPLE VOLUME in $\mathrm{mL}$ & 0.100 & 0.100 \\
\hline Hestogoge & SAMPLE DILUTION FACTOR (Spiked Vial) & 1. & 1 \\
\hline @ALPHA01 & DIGEST GRAMS of SOLIDS / L & 2.0108 & 2.0108 \\
\hline Nuth & SPIKE YOLUME in $\mathrm{mL}$ & 0.100 & 0.100 \\
\hline SOLID & SPIKE DILUTION FACTOR & 1 & 1 \\
\hline 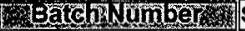 & SPIKE BOOK NUMBER & $131 \mathrm{B43}$ & $131 \mathrm{~B} 43$ \\
\hline 97003525 & SPIKE VALUE in $\mu \mathrm{Ci} / \mathrm{mL}$ & $2.6286 \mathrm{E}-02$ & $2.6286 \mathrm{E}-02$ \\
\hline REOH S & INSTRUMENT EFFICIENCY FACTOR & 0.2701 & 0.2701 \\
\hline 0 & SAMPLE + SPIKE $\mu$ Ci/g & $8.56 \mathrm{E}+00$ & $8.63 E+00$ \\
\hline WSanolepere & AVERAGE or MAXIMUM $\mu$ CI/g in SAMPLE & $2.7370 \mathrm{E}-03$ & \\
\hline FUSIONO1 & & & \\
\hline 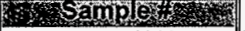 & & & \\
\hline S97T001363 & Rs (Sample Count Rate) $=(\mathrm{TC} / \mathrm{CT})-\mathrm{BKG}$ & & \\
\hline Lstrumentoger & $=R s * 1000 \mathrm{~mL} / \mathrm{L} * \mathrm{DF} /\left(\mathrm{EFF}{ }^{*} \mathrm{SS}\right.$ & $\mathrm{Dg} / \mathrm{L}{ }^{\star} 2220000$ & $\mathrm{dpm} / \mu \mathrm{Ci})$ \\
\hline WB26872 & QC ACTUAL = SVal & & \\
\hline 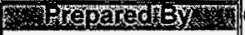 & QC FOUND $=\left(\left((S+S \mu C i / g-S A M P L E \mu C i / g) *\left(\left(S D F /(S V O)^{*} 10\right.\right.\right.\right.$ & D)) $/(\mathrm{DF} / \mathrm{SS} / \mathrm{Dg} /$ & Lا))) \\
\hline $\mathbf{S Z C}$ & PERCENT SPIKE RECOVERY = (QC FOUND / QC ACTUAL) $*$ & & \\
\hline 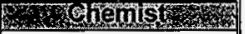 & & & \\
\hline SLF & & & \\
\hline 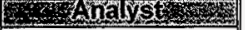 & & & \\
\hline SMF & & & $\cdot$ \\
\hline 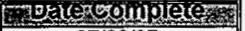 & & & \\
\hline $07 / 23 / 97$ & & & \\
\hline WHaLs & & & \\
\hline $07 / 22 / 97$ & QC ACTUAL & $2.63 E-02$ & \\
\hline Ptodrsis dofe & QCFOUND & 1.73E-02 & \\
\hline 10:00 AM & AVG. PERCENT SPIKE RECOVERY & $65.7 \%$ & \\
\hline $\begin{array}{c}\text { Sample } \\
\text { BY-101 }\end{array}$ & & ' & \\
\hline
\end{tabular}

\begin{tabular}{|c|c|c|}
\hline Analyst: & $S Z C$ & Date: $23-J u l-97$ \\
\hline Signature of Chemist: & SLF & Date: 2370492 \\
\hline
\end{tabular}


HNF-SD-WM-DP-258, REV. 0

APPENDIX A : OPPORTUNISTIC ANALYTE RESULTS 
HNF-SD-WM-DP-258, REV. 0

THIS PAGE INTENTIONALLY LEFT BLANK 
CORE NUMBER: 189

SEGMENT \#: 2

SEGMENT PORTION: L LOWer Half of Segment

\begin{tabular}{|c|c|c|c|c|c|c|c|c|c|c|c|c|}
\hline ample\# & A\# & Analyte & Unit & Standard \% & Blank & Result & Duplicate & Average & RPD \% & Spk Rec \% & Det Limit & Count Err\% \\
\hline S971001358 & $\mathrm{F}$ & silver-ICP-Fusion & ug $/ 9$ & 104.6 & $<1.00 \mathrm{e}-02$ & $<2.09 \mathrm{e}+02$ & $<2.09 e 2$ & $\mathrm{n} / \mathrm{a}$ & $\mathrm{n} / \mathrm{a}$ & 86.10 & 209.0 & $n / a$ \\
\hline S97T001358 & $F$ & Aluminium - [CP-Fusion & $\mathrm{ug} / \mathrm{g}$ & 100.8 & $<5.00 \mathrm{e}-02$ & $3.34 \mathrm{e}+04$ & $3.38 \mathrm{e}+04$ & $3.36 \mathrm{e}+04$ & 1.19 & 102.0 & $1.05 e+03$ & $\mathrm{n} / \mathrm{a}$ \\
\hline \$977001358 & $F$ & Arsenic-ICP-Fusion & $4 \mathrm{~g} / \mathrm{g}$ & 103.8 & $<1.00 e-01$ & $<2.09 \mathrm{e}+03$ & $<2.09 \mathrm{e} 3$ & $\mathrm{n} / \mathrm{a}$ & $n / a$ & 103.0 & $2.09 e+03$ & $n / a$ \\
\hline S97T001358 & $\mathbf{F}$ & Boron-ICP-Fusion & $u g / g$ & 99.80 & $<5.00 \mathrm{e}-02$ & $<1.04 \mathrm{e}+03$ & $<1.05 \mathrm{e}^{3}$ & $\mathrm{n} / \mathrm{a}$ & $\mathrm{n} / \mathrm{a}$ & 95.90 & $1.05 \mathrm{e}+03$ & $\mathrm{n} / \mathrm{a}$ \\
\hline S97T001358 & $F$ & Barium-ICP-Fusion & $\mathrm{ug} / \mathrm{g}$ & 102.2 & $<5.00 \mathrm{e}-02$ & $<1.04 \mathrm{e}+03$ & $<1.05 \mathrm{e} 3$ & $n / a$ & $\mathrm{n} / \mathrm{a}$ & 101.0 & $1.05 e+03$ & $\mathrm{n} / \mathrm{a}$ \\
\hline 5971001358 & $\mathrm{~F}$ & Beryllium-ICP-Fusion & $\operatorname{ug} / 9$ & 103.8 & $<5.00 e-03$ & $<1.04 \mathrm{e}+02$ & $<1.05 \mathrm{e} 2$ & $\mathrm{n} / \mathrm{a}$ & $\mathrm{n} / \mathrm{a}$ & 99.80 & 105.0 & $\mathrm{n} / \mathrm{a}$ \\
\hline S97T001358 & $F$ & Bismuth - ICP-fusion & $\mathrm{ug} / \mathrm{g}$ & 102.6 & $<1.00 e-01$ & $<2.09 \mathrm{e}+03$ & $<2.09 \mathrm{e}^{3}$ & $n / a$ & $\mathrm{n} / \mathrm{a}$ & 102.0 & $2.09 \mathrm{e}+03$ & $\mathrm{n} / \mathrm{a}$ \\
\hline S97T001358 & $F$ & Calcium-ICP-Fusion & ug/g & 97.80 & $<1.00 \mathrm{e}-01$ & $<2,09 \mathrm{e}+03$ & $<2.09 \mathrm{e} 3$ & $n / a$ & $n / a$ & 98.70 & $2.09 e+03$ & $\mathrm{n} / \mathrm{a}$ \\
\hline \$97T 001358 & $\mathbf{F}$ & Cadmium-ICP-Fusion & ug/g & 101.0 & $<5.00 e-03$ & $1.08 \mathrm{e}+02$ & 146.0 & 127.0 & 29.9 & 100.0 & 105.0 & $\mathrm{n} / \mathrm{a}$ \\
\hline \$977001358 & $\mathbf{F}$ & Cerium-ICP-Fusion & $u g / g$ & 100.2 & $<1.00 e^{-01}$ & $<2,09 \mathrm{e}+03$ & $<2.09 \mathrm{e} 3$ & $\mathrm{n} / \mathrm{a}$ & $n / a$ & 106.0 & $2.09 e+03$ & $n / a$ \\
\hline s97r001358 & $\vec{F}$ & Cobalt - ICP-fusion & $\mathrm{ug} / \mathrm{g}$ & 99.80 & $<2.00 e-02$ & $<4.18 \mathrm{e}+02$ & $<4.18 \mathrm{e} 2$ & $n / a$ & $n / a$ & 101.0 & 418.0 & $\mathrm{n} / \mathrm{a}$ \\
\hline 5971001358 & $F$ & Chromium - ICP-Fusion & ug/g & 100.4 & $<1.00 e-02$ & $1.21 \mathrm{e}+04$ & $1.29 \mathrm{e}+04$ & $1.25 e+04$ & 6.40 & 104.0 & 209.0 & $\mathrm{n} / \mathrm{a}$ \\
\hline \$971001358 & $F$ & Copper-ICP-Fusion & ug/g & 103.8 & $<1.00 \mathrm{e}-02$ & $<2.09 e+02$ & $<2.09 \mathrm{e} 2$ & $n / a$ & $\mathrm{n} / \mathrm{a}$ & 103.0 & 209.0 & $\mathrm{n} / \mathrm{a}$ \\
\hline S97T001358 & $F$ & Iron-ICP-Fusion & ug/g & 99.20 & $<5.00 e-02$ & $<1.04 \mathrm{e}+03$ & $1.21 \mathrm{e}+03$ & $n / a$ & $n / a$ & 103.0 & $1.05 e+03$ & $\mathrm{n} / \mathrm{a}$ \\
\hline S97T 001358 & $\bar{F}$ & Lanthanum-ICP-Fusion & $\operatorname{ug} / 9$ & 101.4 & $<5.00 e-02$ & $<1.04 \mathrm{e}+03$ & $<1.05 \mathrm{e} 3$ & $n / a$ & $n / a$ & 100.0 & $1.05 e+03$ & $n / a$ \\
\hline S97T001358 & $F$ & Magnesium -ICP-Fusion & $u g / g$ & 97.40 & $<1.00 \mathrm{e}-01$ & $<2.09 e+03$ & $<2.09 \mathrm{e}$ & $n / a$ & $n / a$ & 96.90 & $2.09 \mathrm{e}+03$ & $\mathrm{n} / \mathrm{a}$ \\
\hline \$97T 001358 & $F$ & Manganese -ICP-Fusion & $u g / g$ & 100.6 & $<1.00 \mathrm{e}-02$ & $<2.09 \mathrm{e}+02$ & $<2.09 \mathrm{e} 2$ & $\mathrm{n} / \mathrm{a}$ & $\mathrm{n} / \mathrm{a}$ & 101.0 & 209.0 & $n / a$ \\
\hline 5979001358 & $F$ & Molybdenum-ICP-Fusion & ug/g & 100.2 & $<5.00 e-02$ & $<1.04 \mathrm{e}+03$ & $<1.05 \mathrm{e} 3$ & $n / a$ & $\mathrm{n} / \mathrm{a}$ & 101.0 & $1.05 \mathrm{e}+03$ & $n / a$ \\
\hline S97T001358 & $\mathrm{F}$ & Sodium-ICP-Fusion & $\mathrm{ug} / \mathrm{g}$ & 100.6 & $<1.00 \mathrm{e}-01$ & $2.44 \mathrm{e}+05$ & $2.30 \mathrm{e}+05$ & $2.37 \mathrm{e}+05$ & 5.91 & 25.80 & $2.09 e+03$ & $\mathrm{n} / \mathrm{a}$ \\
\hline 5977001358 & $F$ & Neodymium - ICP-Fusion & $\mathrm{ug} / \mathrm{g}$ & 103.6 & $<1.00 \mathrm{e}-01$ & $<2.09 \mathrm{e}+03$ & $<2.09 \mathrm{e} 3$ & $n / a$ & $\mathrm{n} / \mathrm{a}$ & 102.0 & $2.09 \mathrm{e}+03$ & $n / a$ \\
\hline S971001358 & $F$ & Phosphorus -ICP-Fusion & $\mathrm{ug} / \mathrm{g}$ & 99.20 & $<2.00 \mathrm{e}-01$ & $2.24 \mathrm{e}+04$ & $2.33 \mathrm{e}+04$ & $2.28 \mathrm{e}+04$ & 3.94 & 101.0 & $4.18 \mathrm{e}+03$ & $n / a$ \\
\hline S97T001358 & $F$ & Lead-ICP-fusion & $u g / 9$ & 101.4 & $<1.00 \mathrm{e}-01$ & $<2.09 e+03$ & $<2.09 \mathrm{e} 3$ & $n / a$ & $n / a$ & 105.0 & $2.09 \mathrm{e}+03$ & $n / a$ \\
\hline S97T001358 & $\dot{\mathbf{F}}$ & Sulfur - ICP-Fusion & ug $/ g$ & 96.60 & $<1.00 e-01$ & $<2.09 \mathrm{e}+03$ & $<2.09 e^{3}$ & $n / a$ & $\mathrm{n} / \mathrm{a}$ & 94.70 & $2.09 \mathrm{e}+03$ & $\mathrm{n} / \mathrm{a}$ \\
\hline 5977001358 & $F$ & Ant imony -ICP-Fusion & $\mathrm{ug} / \mathrm{g}$ & 94.20 & $<6.00 \mathrm{e}-02$ & $<1.25 \mathrm{e}+03$ & $<1.26 \mathrm{e} 3$ & $\mathrm{n} / \mathrm{a}$ & $\mathrm{n} / \mathrm{a}$ & 97.20 & $1.25 e+03$ & $\mathrm{n} / \mathrm{a}$ \\
\hline $597 T 001358$ & $F$ & Silicon-ICP-Fusion & $\mathrm{ug} / \mathrm{g}$ & 96.60 & $<5.00 e^{-02}$ & $7.43 e+03$ & $8.45 e+03$ & $7.94 \mathrm{e}+03$ & 12.8 & 98.20 & $1.05 \mathrm{e}+03$ & $n / a$ \\
\hline 5971001358 & $F$ & Samarium-ICP-Fusion & $\mathrm{ug} / \mathrm{g}$ & 103.0 & $<1.00 e-01$ & $<2.09 \mathrm{e}+03$ & $<2.09 \mathrm{e} 3$ & $n / a$ & $\mathrm{n} / \mathrm{a}$ & 104.0 & $2.09 \mathrm{e}+03$ & $n / a$ \\
\hline$\overline{S 971001358}$ & $F$ & strontium $-[\mathrm{ICP}-$ Fusion & $\mathrm{ug} / \mathrm{g}$ & 101.0 & $<1.00 e-02$ & $<2.09 \mathrm{e}+02$ & $<2.09 \mathrm{e} 2$ & $n / a$ & $n / a$ & 101.0 & 209.0 & $n / a$ \\
\hline S97T001358 & $\mathbf{F}$ & İtanium-ICP-Fusion & ug/g & 97.40 & $<1.00 e-02$ & $<2.09 e+02$ & $<2.09 \mathrm{e} 2$ & $n / a$ & $n / a$ & 97.70 & 209.0 & $n / a$ \\
\hline 5979001358 & $F$ & Thallium-ICP-Fusion & ug/g & 102.4 & $<2.00 e-01$ & $<4.18 \mathrm{e}+03$ & $<4.18 \mathrm{e} 3$ & $\mathrm{n} / \mathrm{a}$ & $\mathrm{n} / \mathrm{a}$ & 100.0 & $4.18 \mathrm{e}+03$ & $n / a$ \\
\hline $597 T 001358$ & $F$ & Uranium -ICP-Fusion & $\mathrm{ug} / \mathrm{g}$ & 93.30 & $<5.00 e-01$ & $<1.04 \mathrm{e}+04$ & $<1.05 \mathrm{e} 4$ & $n / a$ & $n / a$ & 82.00 & $1.05 e+04$ & $n / a$ \\
\hline 5974001358 & $F$ & Vanadium-ICP-fusion & ug/g & 99.20 & $<5.00 \mathrm{e}-02$ & $<1.04 \mathrm{e}+03$ & $<1.05 \mathrm{e} 3$ & $n / a$ & $n / a$ & 100.0 & $9.05 e+0 \overline{3}$ & $n / a$ \\
\hline 5971001358 & $F$ & Zinc-ICP-Fusion & $\mathrm{ug} / \mathrm{g}$ & 101.2 & $<1.00 e-02$ & $<2.09 \mathrm{e}+02$ & $<2.09 \mathrm{e} 2$ & $\mathrm{n} / \mathrm{a}$ & $n / a$ & 101.0 & 209.0 & $n / a$ \\
\hline S97T001358 & $F$ & Zirconium - ICP-Fusion & $u g / g$ & 98.00 & $<1.00 e-02$ & $<2.09 \mathrm{e}+02$ & $<2.09 \mathrm{e} 2$ & $n / a$ & $n / a$ & 97.70 & 209.0 & $\mathrm{n} / \mathrm{a}$ \\
\hline$\$ 971001360$ & w & Fluoride-IC-Dionex $4000 / 4500$ & $\operatorname{ug} / 9$ & 112.4 & $<1.20 e-02$ & $4.13 e+03$ & $6.13 e+03$ & $5.13 \mathrm{e}+03$ & 39.0 & 88.14 & 120.9 & $\mathrm{n} / \mathrm{a}$ \\
\hline 5971001360 & $W$ & Chloride-IC-Dionex $4000 / 4500$ & ug/g & 93.04 & $<1.70 \mathrm{e}-02$ & $<1.71 \mathrm{e}+02$ & $<1.73 \mathrm{e} 2$ & $\mathrm{n} / \mathrm{a}$ & $\mathrm{n} / \mathrm{a}$ & 90.51 & 171.3 & $n / a$ \\
\hline $597 T 001360$ & $\mathrm{~W}$ & Nitrite-IC - Dionex $4000 / 4500$ & $\mathrm{ug} / \mathrm{g}$ & 94.30 & $2.26 \mathrm{e}-01$ & $<1.09 \mathrm{e}+03$ & $<1.10 \mathrm{e}^{3}$ & $n / a$ & $\mathrm{n} / \mathrm{a}$ & 92.83 & $1.09 e+03$ & $\mathrm{n} / \mathrm{a}$ \\
\hline 5977001360 & W & Nitrate by $[C=D$ ionex $4000 / 4500$ & ug $/ g$ & 93.27 & $<1.39 \mathrm{e}-01$ & $4.11 \mathrm{e}+05$ & $3.27 \mathrm{e}+05$ & $3.69 \mathrm{e}+05$ & 22.8 & 38.38 & $1.40 \mathrm{e}+\overline{03}$ & $\mathrm{n} / \mathrm{a}$ \\
\hline $597 \% 001360$ & $\bar{W}$ & Phosphate-IC-D ionex $4000 / 4500$ & $\mathrm{ug} / \mathrm{g}$ & 98.90 & $<1.20 e^{-01}$ & $4.48 e+04$ & $6.45 e+04$ & $5.47 e^{+04}$ & 36.0 & 92.66 & $1.21 \mathrm{e}+03$ & $n / a$ \\
\hline S97T001360 & W. & Sulfate by IC-D ionex $4000 / 4500$ & ug/g & 93.04 & $<1.38 \mathrm{e}-01$ & $<1.39 \mathrm{e}+03$ & $<1.41 \mathrm{e} 3$ & $\mathrm{n} / \mathrm{a}$ & $n / a$ & 91.93 & $1.39 \mathrm{e}+03$ & $n / a$ \\
\hline S9710001360 & $\mathrm{W}$ & Oxalate-IC-Dionex $4000 / 4500$ & $\log / g$ & 94.93 & $<1.05 e-01$ & $7.85 e+03$ & $7.94 \mathrm{e}+03$ & $7.89 \mathrm{e}+03$ & 1.14 & 93.06 & $1.06 \mathrm{e}+03$ & $n / a$ \\
\hline
\end{tabular}


Appendix A. Opportunistic Analyses

BY-101

CORE NUMBER: 199

SEGMENT \#: Field Blank

SEGMENT PORTION: Field Blank

\begin{tabular}{|c|c|c|c|c|c|c|c|c|c|c|c|c|}
\hline Sample\# & A\# & Analyte & Unit & Standard \% & Blank & Result & Duplicate & Average & RPD \% & Spk Rec \% & Det Limit & Count Err\% \\
\hline 5971001365 & $D$ & Silver-ICP-Acid Dil. & $\mathrm{ug} / \mathrm{mL}$ & 102.2 & $<1.00 \mathrm{e}-02$ & $<2.00 \mathrm{e}-02$ & $<2.00 \mathrm{e}-2$ & $n / a$ & $\mathrm{n} / \mathrm{a}$ & $n / a$ & $2.00 \mathrm{e}-02$ & $n / a$ \\
\hline 5977001365 & $D$ & Aluminium-ICP-Acid Dil. & $\mathrm{ug} / \mathrm{mL}$ & 101.0 & $<5.00 \mathrm{e}-02$ & 4.130 & 4.150 & 4.140 & 0.48 & $\mathrm{n} / \mathrm{a}$ & $1.00 \mathrm{e}-01$ & $\mathrm{n} / \mathbf{a}$ \\
\hline 5977001365 & $D$ & Arsenic-ICP-Acid Dil. & ug/mL & 106.6 & $<1.00 \mathrm{e}-01$ & $<2.00 \mathrm{e}-01$ & $<2.00 \mathrm{e}-1$ & $\mathrm{n} / \mathrm{a}$ & $\mathrm{n} / \mathrm{a}$ & $n / a$ & $2.00 e-01$ & $\mathrm{n} / \mathrm{a}$ \\
\hline$\$ 971001365$ & D & Boron-ICP-ACid Dil. & $\mathrm{ug} / \mathrm{mL}$ & 103.4 & $<5.00 \mathrm{e}-02$ & 1.260 & 1.230 & 1.245 & 2.41 & $n / a$ & $1.00 \mathrm{e}-01$ & $\mathrm{n} / \mathrm{a}$ \\
\hline$\$ 977001365$ & D & Barium-ICP-Acid Dil. & $\mathrm{ug} / \mathrm{mL}$ & 103.8 & $<5.00 e-02$ & $<1.00 \mathrm{e}-01$ & $<1.00 e^{-1}$ & $n / a$ & $n / a$ & $n / a$ & $1.00 e-01$ & $\mathrm{n} / \mathrm{a}$ \\
\hline 5971001365 & D & Beryllium-ICP-Acid Dil. & $\mathrm{ug} / \mathrm{mL}$ & 107.0 & $<5.00 \mathrm{e}-03$ & $<1.00 \mathrm{e}-02$ & $<1.00 \mathrm{e}-2$ & $\mathrm{n} / \mathrm{a}$ & $n / a$ & $n / a$ & $1.00 \mathrm{e}-02$ & $\mathrm{n} / \mathrm{a}$ \\
\hline$\$ 977001365$ & $D$ & Bismuth-ICP-Acid Dil. & $\mathrm{ug} / \mathrm{mL}$ & 105.2 & $<1.00 \mathrm{e}-01$ & $2.00 \mathrm{e}-01$ & $<2.00 e^{-1}$ & $\mathrm{n} / \mathrm{a}$ & $n / a$ & $n / a$ & $2.00 \mathrm{e}-01$ & $\mathrm{n} / \mathrm{a}$ \\
\hline$\$ 977001365$ & $D$ & Calcium-ICP-Acid Dil. & $\mathrm{ug} / \mathrm{mL}$ & 104.6 & $<1.00 \mathrm{e}-01$ & $<2.00 \mathrm{e}-01$ & $<2.00 \mathrm{e}-1$ & $\mathrm{n} / \mathrm{a}$ & $n / a$ & $n / a$ & $2.00 \mathrm{e}-01$ & $\mathrm{n} / \mathrm{a}$ \\
\hline 5971001365 & D & Cadmi um-ICP-Acid Dil. & $\mathrm{ug} / \mathrm{mL}$ & 102.8 & $<5.00 \mathrm{e}-03$ & $<1.00 \mathrm{e}-02$ & $<1.00 e-2$ & $\mathrm{n} / \mathrm{a}$ & $\mathrm{n} / \mathrm{a}$ & $\mathrm{n} / \mathrm{a}$ & $1.00 \mathrm{e}-02$ & $\mathrm{n} / \mathrm{a}$ \\
\hline$\$ 971001365$ & $D$ & Cerium-ICP-Acid Dil. & $\underline{\mathrm{ug} / \mathrm{mL}}$ & 103.4 & $<1.00 \mathrm{e}-01$ & $<2.00 e-01$ & $<2.00 e^{-1}$ & $\mathrm{n} / \mathrm{a}$ & $n / a$ & $n / a$ & $2.00 \mathrm{e}-01$ & $n / a$ \\
\hline 5971001365 & D & Cobalt-ICP-Acid Dil. & $\mathrm{ug} / \mathrm{mL}$ & 102.2 & $<2.00 e-02$ & $<4.00 \mathrm{e}-02$ & $<4.00 \mathrm{e}-2$ & $\mathrm{n} / \mathrm{a}$ & $n / a$ & $n / a$ & $4.00 \mathrm{e}-02$ & $\mathrm{n} / \mathrm{a}$ \\
\hline 5971001365 & $D$ & Chromium-ICP-Acid Dil. & $\mathrm{ug} / \mathrm{mL}$ & 103.8 & $<1.00 \mathrm{e}-02$ & $3.14 \mathrm{e}-02$ & $3.27 e-02$ & $3.20 \mathrm{e}-02$ & 4.06 & $\mathrm{n} / \mathrm{a}$ & $2.00 e-02$ & $\mathrm{n} / \mathrm{a}$ \\
\hline 5977001365 & D & Copper-ICP-Acid Dil. & $\mathrm{ug} / \mathrm{mL}$ & 100.2 & $<1.00 \mathrm{e}-02$ & $<2.00 e^{-02}$ & $<2.00 e+2$ & $\mathrm{n} / \mathrm{a}$ & $n / a$ & $n / a$ & $2.00 \mathrm{e}-02$ & $\mathrm{n} / \mathrm{a}$ \\
\hline 597 T001365 & $D$ & Iron-ICP-ACId Dil. & $\underline{\mathrm{ug} / \mathrm{ML}}$ & 97.00 & $<5.00 \mathrm{e}-02$ & $<1.00 \mathrm{e}-01$ & $<1.00 \mathrm{e}-1$ & $n / a$ & $n / a$ & $\mathrm{n} / \mathrm{a}$ & $1.00 \mathrm{e}-01$ & $\mathrm{n} / \mathrm{a}$ \\
\hline S97T001365 & $D$ & Potass ium-ICP-Acid Dil. & $\mathrm{ug} / \mathrm{mL}$ & 100.2 & $<5.00 e^{-01}$ & 1.340 & 1.190 & 1.265 & 11.9 & $\mathrm{n} / \mathrm{a}$ & 1.000 & $\mathrm{n} / \mathrm{a}$ \\
\hline 5971001365 & $D$ & Lanthanum-ICP-Acid Dil. & ug/mL & 105.0 & $<5.00 \mathrm{e}-02$ & $<1.00 \mathrm{e}-01$ & $<1.00 \mathrm{e}-1$ & $\mathrm{n} / \mathrm{a}$ & $\mathrm{n} / \mathrm{a}$ & $n / a$ & $1.00 \mathrm{e}-01$ & $\mathrm{n} / \mathrm{a}$ \\
\hline 5971001365 & D & Magnes i um-ICP-Acid Dil. & $\mathrm{ug} / \mathrm{mL}$ & 105.8 & $<1.00 e^{-01}$ & $<2.00 e^{-01}$ & $<2.00 e^{-1}$ & $\mathrm{n} / \mathrm{a}$ & $\mathrm{n} / \mathrm{a}$ & $n / a$ & $2.00 e-01$ & $n / a$ \\
\hline 5971001365 & $D$ & Manganese-ICP-Acid Dil. & $\mathrm{ug} / \mathrm{mL}$ & 100.8 & $<1.00 \mathrm{e}-02$ & $<2.00 \mathrm{e}-02$ & $<2.00 \mathrm{e}-2$ & $\mathrm{n} / \mathrm{a}$ & $n / a$ & $n / a$ & $2.00 \mathrm{e}-02$ & $\mathrm{n} / \mathrm{a}$ \\
\hline $597 T 001365$ & $D$ & Molybdenum-ICP-Acid Dil. & $u g / m L$ & 102.6 & $<5.00 \mathrm{e}-02$ & $<1.00 e^{-01}$ & $<1.00 \mathrm{e}-1$ & $\mathrm{n} / \mathrm{a}$ & $\mathrm{n} / \mathrm{a}$ & $\mathrm{n} / \mathrm{a}$ & $1.00 \mathrm{e}-01$ & $n / a$ \\
\hline 5971001365 & D & Sodium-ICP-Acid Dil. & ug/int & 100.8 & $<1.00 \mathrm{e}-01$ & 31.80 & 32.00 & 31.90 & 0.63 & $n / a$ & $2.00 e-01$ & $\mathrm{n} / \mathrm{a}$ \\
\hline 5971001365 & $D$ & Neodymi um-ICP-Acid Dil. & $\mathrm{ug} / \mathrm{mL}$ & 105.2 & $<1.00 \mathrm{e}-01$ & $<2.00 \mathrm{e}-01$ & $<2,00 e^{-1}$ & $\mathrm{n} / \mathrm{a}$ & $n / a$ & $\mathrm{n} / \mathrm{a}$ & $2.00 e-01$ & $n / a$ \\
\hline 5971001365 & D & Nickel-ICP-Acid Dil. & ug/mL & 102.8 & $<2.00 \mathrm{e}-02$ & $<4.00 \mathrm{e}-02$ & $<4.00 \mathrm{e}-2$ & $\mathrm{n} / \mathrm{a}$ & $n / a$ & $\mathrm{n} / \mathrm{a}$ & $4.00 \mathrm{e}-02$ & $n / a$ \\
\hline 5971001365 & D & Phosphorus-ICP-Acid Dil. & $\mathrm{ug} / \mathrm{mL}$ & 103.6 & $<2.00 e-01$ & $4.36 \mathrm{e}-01$ & $4.16 \mathrm{e}-01$ & $4.26 \mathrm{e}-01$ & 4.69 & $n / a$ & $4.00 e-01$ & $n / a$ \\
\hline 5971001365 & D & Lead-ICP-Acid Dil. & $\mathrm{ug} / \mathrm{mL}$ & 104.0 & $<1.00 e-01$ & $<2.00 e^{-01}$ & $<2.00 e^{-1}$ & $\mathrm{n} / \mathrm{a}$ & $\mathrm{n} / \mathrm{a}$ & $n / a$ & $2.00 \mathrm{e}-01$ & $n / a$ \\
\hline 5971001365 & D & Sulfur-ICP-Acid Dil. & $u g / m L$ & 101.2 & $<1.00 \mathrm{e}-01$ & 1.090 & 1.080 & 1.085 & 0.92 & $\mathrm{n} / \mathrm{a}$ & $2.00 e+01$ & $\mathrm{n} / \mathrm{a}$ \\
\hline 5977001365 & $\bar{D}$ & Antimony-ICP-Acid Dil. & $\mathrm{ug} / \mathrm{mL}$ & 97.80 & $<6.00 e-02$ & $<1.20 \mathrm{e}-01$ & $<1.20 \mathrm{e}-1$ & $\mathrm{n} / \mathrm{a}$ & $n / a$ & $n / a$ & $1.20 \mathrm{e}-01$ & $n / a$ \\
\hline 5971001365 & D & Selenium-ICP-Acid Dil. & $\mathrm{ug} / \mathrm{mL}$ & 100.4 & $<1.00 \mathrm{e}-01$ & $<2.00 \mathrm{e}-01$ & $<2.00 e^{-1}$ & $n / a$ & $n / a$ & $n / a$ & $2.00 e^{-01}$ & $\mathrm{n} / \mathrm{a}$ \\
\hline $597 T 001365$ & $D$ & Silicon-ICP-ACid Dil. & $\mathrm{ug} / \mathrm{ml}$ & 98.80 & $<5.00 \mathrm{e}-02$ & 2.150 & 2.140 & 2.145 & 0.47 & $n / a$ & $1.00 \mathrm{e}-01$ & $\mathrm{n} / \mathrm{a}$ \\
\hline 5971001365 & D & Samarium-ICP-Acid Dil. & $\mathrm{ug} / \mathrm{ml}$ & 102.0 & $<1.00 \mathrm{e}-01$ & $<2.00 \mathrm{e}-01$ & $\leq 2.00 \mathrm{e}-1$ & $\mathrm{n} / \mathrm{a}$ & $\mathrm{n} / \mathrm{a}$ & $\mathrm{n} / \mathrm{a}$ & $2.00 \mathrm{e}-01$ & n/a \\
\hline 5971001365 & D & Strontium-ICP-Acid Dil. & $\mathrm{ug} / \mathrm{mL}$ & 102.8 & $\leq 1.00 \mathrm{e}-02$ & $<2.00 \mathrm{e}-02$ & $<2.00 \mathrm{e}-2$ & $n / a$ & $\mathrm{n} / \mathrm{a}$ & $\mathrm{n} / \mathrm{a}$ & $2.00 e-02$ & $n / a$ \\
\hline 5971001365 & D & Iitanium-ICP-Acid Dil. & $\mathrm{ug} / \mathrm{ml}$ & 100.6 & $<1.00 \mathrm{e}-02$ & $<2.00 e-02$ & $<2.00 e^{-2}$ & $n / a$ & $\mathrm{n} / \mathrm{a}$ & $\mathrm{n} / \mathrm{a}$ & $2.00 \mathrm{e}-02$ & $n / a$ \\
\hline $597 T 001365$ & D & Thallium-ICP-Acid Dil. & $\mathrm{ug} / \mathrm{ml}$ & 100.4 & $<2.00 e^{-0} 01$ & $<4.00 \mathrm{e}-01$ & $<4.00 \mathrm{e}-1$ & n/a & $n / a$ & $\mathrm{n} / \mathrm{a}$ & $4.00 e-01$ & $\mathrm{n} / \mathrm{a}$ \\
\hline S97T001365 & D & Uranium-ICP-Acid Dil, & ug/ml & 99.30 & $<5.00 \mathrm{e}-01$ & 1.000 & $<1.00 \mathrm{e} 0$ & $\mathrm{n} / \mathrm{a}$ & $n / a$ & $\mathrm{n} / \mathrm{a}$ & 1.000 & $n / a$ \\
\hline$\$ 971$ & D & Vanadium-ICP-Acid Dil. & $\mathrm{ug} / \mathrm{ml}$ & 101.0 & $<5.00 \mathrm{e}-02$ & $<1.00 \mathrm{e}-01$ & $<1.00 e-1$ & $\mathrm{n} / \mathrm{a}$ & $n / a$ & $n / a$ & $1.00 e-01$ & $n / a$ \\
\hline 5971001365 & D & Zinc-ICP-Acid Dil. & $\mathrm{ug} / \mathrm{mL}$ & 97.40 & $<1.00 e^{-02}$ & $2.53 \mathrm{e}-02$ & $2.88 \mathrm{e}-02$ & $2.70 \mathrm{e}-02$ & 12.9 & $\mathrm{n} / \mathrm{a}$ & $2.00 e^{-02}$ & $\mathrm{n} / \mathrm{a}$ \\
\hline S971001365 & D & Zirconium-ICP-Acid Dil. & ug/mL & 100.8 & $<1.00 \mathrm{e}-02$ & $<2.00 e-02$ & $<2.00 \mathrm{e}-2$ & $\mathrm{n} / \mathrm{a}$ & $\mathrm{n} / \mathrm{a}$ & $\mathrm{n} / \mathrm{a}$ & $2.00 e-02$ & $\mathrm{n} / \mathrm{a}$ \\
\hline$\$ 971001365$ & & Fluoride-IC-D ionex $4000 / 4500$ & $\mathrm{ug} / \mathrm{ml}$ & 92.03 & $<1.20 \mathrm{e}-02$ & $1.61 e^{-01}$ & $1.61 e^{-01}$ & $1.61 \mathrm{e}^{-01}$ & 0.00 & $n / a$ & $1.20 \mathrm{e}-02$ & $\mathrm{n} / \mathrm{a}$ \\
\hline$\$ 9710$ & & Chloride-IC-Dionex $4000 / 4500$ & ug/mL & 95.06 & $2.70 \mathrm{e}-02$ & $7.37 \mathrm{e}-01$ & $7.32 e^{-0} 01$ & $7.34 \mathrm{e}-01$ & 0.68 & $\mathrm{n} / \mathrm{a}$ & $1.70 e-02$ & $\mathrm{n} / \mathrm{a}$ \\
\hline 5971001365 & & Nitrite-IC - Dionex $4000 / 4500$ & $\mathrm{ug} / \mathrm{mL}$ & 95.21 & $<1.08 \mathrm{e}-01$ & 8.729 & 8.710 & 8.720 & 0.23 & $n / a$ & $1.08 \mathrm{e}-01$ & $\mathrm{n} / \mathrm{a}$ \\
\hline 5977001365 & & Nitrate by IC-Dionex $4000 / 4500$ & $\mathrm{ug} / \mathrm{mL}$ & 96.47 & $1.53 e-01$ & 27.16 & 26.90 & 27.03 & 1.11 & $n / a$ & $1.39 e-01$ & $\mathrm{n} / \mathrm{a}$ \\
\hline S97T001365 & & Phosphate-IC-Dionex $4000 / 4500$ & $\mathrm{ug} / \mathrm{mL}$ & 103.9 & $<1.20 \mathrm{e}-01$ & 1.257 & 1.220 & 1.238 & 3.23 & $\mathrm{n} / \mathrm{a}$ & $1.20 \mathrm{e}-01$ & $\mathrm{n} / \mathrm{a}$ \\
\hline $597 \mathrm{~T}$ & & Sulfate by IC-Dionex $4000 / 4500$ & $\mathrm{ug} / \mathrm{mL}$ & 100.9 & $1.90 \mathrm{e}-01$ & $3.26 e^{-01}$ & $3.36 e-01$ & $3.31 \mathrm{e}-01$ & 3.02 & $n / a$ & $1.38 \mathrm{e}-01$ & $\mathrm{n} / \mathrm{a}$ \\
\hline$\$ 9971001365$ & & Dxalate-IC-Dionex $4000 / 450$ & $\mathrm{ug} / \mathrm{mL}$ & 93.21 & $<1.05 \mathrm{e}-01$ & $<1.05 \mathrm{e}-01$ & $<1.05 e^{-1}$ & $n / a$ & $\mathrm{n} / \mathrm{a}$ & $\mathrm{n} / \mathrm{a}$ & $1.05 e-01$ & $\mathrm{n} / \mathrm{a}$ \\
\hline
\end{tabular}


Appendix A. Opportunistic Analyses BY-101

CORE NUMBER: 199

SEGMENT \#: $n$ /a

SEGMENT PORTION: Hydrostatic Head Fluid

\begin{tabular}{|c|c|c|c|c|c|c|c|c|c|c|c|c|}
\hline Sample\# & \begin{tabular}{l|l}
$\mathrm{R}$ & $\mathrm{A \#}$ \\
\end{tabular} & Analyte & Unit & Standard \% & Blank & Result & Duplicate & Average & RPD \% & Spk Rec \% & Det Limit & Count Err\% \\
\hline 5971001241 & $D$ & Silver-ICP-Acid Dil. & $u g / \mathrm{mL}$ & 98.00 & $<1.00 \mathrm{e}-02$ & $<2.00 \mathrm{e}-01$ & $<2.00 e^{-1}$ & $n / a$ & $n / a$ & $n / a$ & $2.00 \mathrm{e}-01$ & $n / a$ \\
\hline S97T001241 & $D$ & Aluminium-ICP-Acid Dil. & $4 \mathrm{~g} / \mathrm{mL}$ & 97.80 & $<5.00 \mathrm{e}-02$ & 1.000 & $<1.00 \mathrm{e} 0$ & $\mathrm{n} / \mathrm{a}$ & $n / a$ & $n / a$ & 1.000 & $n / a$ \\
\hline 5977001241 & D & Arsenic-1CP-ACid Dil. & $\mathrm{ug} / \mathrm{mL}$ & 104.2 & $<1.00 e^{-01}$ & 2.000 & $<2.00 \mathrm{e} 0$ & $\mathrm{n} / \mathrm{a}$ & $\mathrm{n} / \mathrm{a}$ & $\mathrm{n} / \mathrm{a}$ & 2.000 & $n / a$ \\
\hline S97T001241 & $D$ & Boron-ICP-ACID Dil. & $\mathrm{ug} / \mathrm{mL}$ & 101.2 & $<5.00 \mathrm{e}-02$ & 1.000 & $<1.00 \mathrm{e} 0$ & $n / a$ & $\mathrm{n} / \mathrm{a}$ & $n / a$ & 1.000 & $n / a$ \\
\hline \$97T001241 & $D$ & Barium-ICP-ACId Dit. & $\mathrm{ug} / \mathrm{mL}$ & 99.40 & $<5.00 \mathrm{e}-02$ & 1.000 & $<1.00 \mathrm{e} 0$ & $n / a$ & $\mathrm{n} / \mathrm{a}$ & $\mathrm{n} / \mathrm{a}$ & 1.000 & $n / a$ \\
\hline S97T001241 & $D$ & Beryllium-ICP-Acid Dil. & $\mathrm{ug} / \mathrm{mL}$ & 102.4 & $<5.00 \mathrm{e}-03$ & $<1.00 \mathrm{e}-01$ & $<1.00 \mathrm{e}-1$ & $n / a$ & $\mathrm{n} / \mathrm{a}$ & $\mathrm{n} / \mathrm{a}$ & $1.00 e-01$ & $n / a$ \\
\hline 5971001241 & $D$ & Bismuth-ICP-Acid Dil. & $\mathrm{ug} / \mathrm{mL}$ & 101.4 & $<1.00 \mathrm{e}-01$ & $<\quad 2.000$ & $<2.00 \mathrm{e} 0$ & $n / a$ & $\mathrm{n} / \mathrm{a}$ & $n / a$ & 2.000 & $n / a$ \\
\hline S97T001241 & D & Calcium-ICP-Acid Dil. & $\mathrm{ug} / \mathrm{ml}$ & 98.40 & $<1.00 \mathrm{e}-01$ & 2.000 & $<2.00 \mathrm{e} 0$ & $n / a$ & $\mathrm{n} / \mathrm{a}$ & $n / a$ & 2.000 & $\mathrm{n} / \mathrm{a}$ \\
\hline S97T001241 & D & Cadmium-ICP-Acid Dil. & $\mathrm{ug} / \mathrm{mL}$ & 99.80 & $<5.00 \mathrm{e}-03$ & $<1.00 \mathrm{e}-01$ & $<1.00 \mathrm{e}-1$ & $n / a$ & $\mathrm{n} / \mathrm{a}$ & $\mathrm{n} / \mathrm{a}$ & $1.00 e-01$ & $n / a$ \\
\hline$\$ 971001241$ & D & Cerium-ICP-Acid Dil. & $\mathrm{ug} / \mathrm{mL}$ & 98.80 & $<1,00 e^{-01}$ & $<2.000$ & $<2.00 e 0$ & $\mathrm{n} / \mathrm{a}$ & $n / a$ & $n / a$ & 2.000 & $n / a$ \\
\hline S97T001241 & D & Cobalt-ICP-Acid Dil. & ug/mL & 101.4 & $<2.00 \mathrm{e}^{-02}$ & $<4.00 e-01$ & $<4.00 \mathrm{e}-1$ & $n / a$ & $n / a$ & $n / a$ & $4.00 \mathrm{e}-01$ & $\mathrm{n} / \mathrm{a}$ \\
\hline S97T001241 & $\bar{D}$ & Chromium-1CP-Acid Dil. & $\mathrm{ug} / \mathrm{mL}$ & 99.20 & $<1.00 e^{-02}$ & $<2,00 \mathrm{e}-01$ & $<2.00 \mathrm{e}-1$ & $n / a$ & $\mathrm{n} / \mathrm{a}$ & $n / a$ & $2.00 e-01$ & $\mathrm{n} / \mathrm{a}$ \\
\hline S97T001241 & $D$ & Copper-ICP-Acid Dil. & ug $/ \mathrm{mL}$ & 98.20 & $<1.00 \mathrm{e}-02$ & $<2.00 e^{-01}$ & $<2.00 e-1$ & $n / a$ & $\mathrm{n} / \mathrm{a}$ & $n / a$ & $2.00 e-01$ & $\mathrm{n} / \mathrm{a}$ \\
\hline$\$ 971001241$ & D & Iron-ICP-ACId Dil. & $\mathrm{ug} / \mathrm{mL}$ & 93.60 & $<5.00 e-02$ & 1.000 & $<1.00 \mathrm{e} 0$ & $n / a$ & $\mathrm{n} / \mathrm{a}$ & $n / a$ & 1.000 & $n / a$ \\
\hline S97T001241 & $D$ & Potassium-ICP-Acid Dil. & $\mathrm{ug} / \mathrm{mL}$ & 101.4 & $<5.00 \mathrm{e}-01$ & 10.00 & $<1,00 \mathrm{e}$ & $\mathrm{n} / \mathrm{a}$ & $n / a$ & $n / a$ & 10.00 & $\mathrm{n} / \mathrm{a}$ \\
\hline \$977001241 & D & Lanthanum-ICP-ACid Dil. & $\mathrm{ug} / \mathrm{mL}$ & 100.6 & $<5.00 \mathrm{e}-02$ & 1.000 & $<1.00 \mathrm{e} 0$ & $n / a$ & $\mathrm{n} / \mathrm{a}$ & $\mathrm{n} / \mathrm{a}$ & 1.000 & $n / a$ \\
\hline S97T001241 & D & Magnes i um-ICP-Acid Dil. & $\mathrm{Ug} / \mathrm{mL}$ & 100.8 & $<1.00 \mathrm{e}-01$ & 2.000 & $<2.00 \mathrm{e} 0$ & $\mathrm{n} / \mathrm{a}$ & $\mathrm{n} / \mathrm{a}$ & $\mathrm{n} / \mathrm{a}$ & 2.000 & $n / a$ \\
\hline S97T001241 & D & Manganese-ICP-Acid Dil. & $\mathrm{Ug} / \mathrm{mL}$ & 97.60 & $<1.00 \mathrm{e}-02$ & $<2.00 \mathrm{e}-01$ & $<2.00 e^{-1}$ & $n / a$ & $\mathrm{n} / \mathrm{a}$ & $n / a$ & $2.00 e-01$ & $\mathrm{n} / \mathrm{a}$ \\
\hline S971001241 & $D$ & Molybdenull-ICP-Acid Dil. & $u \mathrm{~g} / \mathrm{mL}$ & 101.2 & $<5.00 \mathrm{e}-02$ & 1.000 & $<1.00 \mathrm{e} 0$ & $n / a$ & $\mathrm{n} / \mathrm{a}$ & $n / a$ & 1.000 & $\mathrm{n} / \mathrm{a}$ \\
\hline S97T001241 & D & Sodium-ICP-Acid Dil. & $\mathrm{Ug} / \mathrm{mL}$ & 102.8 & $<1.00 e^{-01}$ & 5.330 & 5.310 & 5.320 & 0.38 & $n / a$ & 2.000 & $\mathrm{n} / \mathrm{a}$ \\
\hline S97T001241 & $D$ & Neodymi um-ICP-Acid Dil. & $\mathrm{ug} / \mathrm{mL}$ & 100.6 & $<1.00 \mathrm{e}-01$ & 2.000 & $<2.00 \mathrm{e} 0$ & n/a & $\mathrm{n} / \mathrm{a}$ & $n / a$ & 2.000 & $\mathrm{n} / \mathrm{a}$ \\
\hline S97T001241 & $D$ & Nickel-ICP-Acid Dil. & $\mathrm{ug} / \mathrm{mL}$ & 99.60 & $<2.00 e-02$ & $<4.00 e-01$ & $<4.00 e^{-1}$ & $n / a$ & $\mathrm{n} / \mathrm{a}$ & $n / a$ & $4.00 e-01$ & $n / a$ \\
\hline S97T001241 & D & PhosphorUs-ICP-Acid Dil. & $\mathrm{ug} / \mathrm{mL}$ & 102.2 & $<2.00 e^{-0} 01$ & 4.000 & $<4.00 \mathrm{e} 0$ & $n / a$ & $\mathrm{n} / \mathrm{a}$ & $n / a$ & 4.000 & $n / a$ \\
\hline S971001241 & D & Lead-ICP-Acid Dil. & $\mathrm{ug} / \mathrm{mL}$ & 98.40 & $<1.00 \mathrm{e}-01$ & 2.000 & $<2.00 \mathrm{e} 0$ & $\mathrm{n} / \mathrm{a}$ & $\mathrm{n} / \mathrm{a}$ & $n / a$ & 2.000 & $n / a$ \\
\hline S97T001241 & D & Sulfur-ICP-Acid Dil. & $\mathrm{ug} / \mathrm{mL}$ & 99.80 & $<1.00 \mathrm{e}-01$ & 2.000 & $<2.00 \mathrm{e} 0$ & $\mathrm{n} / \mathrm{a}$ & $n / a$ & $n / a$ & 2.000 & $\mathrm{n} / \mathrm{a}$ \\
\hline S97T001241 & D & Antimony-ICP-Acid Dil. & $\mathrm{ug} / \mathrm{mL}$ & 96.20 & $<6.00 e^{-02}$ & 1.200 & $<1.20 \mathrm{e} 0$ & $\mathrm{n} / \mathrm{a}$ & $\mathrm{n} / \mathrm{a}$ & $n / a$ & 1.200 & $n / a$ \\
\hline \$97T001241 & D & Seleniun-ICP-Acid Dil. & $\mathrm{ug} / \mathrm{mL}$ & 94.60 & $<1.00 \mathrm{e}-01$ & 2.000 & $<2.00 \mathrm{e} 0$ & $n / a$ & $n / a$ & $n / a$ & 2.000 & $\mathrm{n} / \mathrm{a}$ \\
\hline 597T001241 & D & Silicon-ICP-Acid Dil. & $\mathrm{ug} / \mathrm{mL}$ & 96.20 & $<5.00 \mathrm{e}-02$ & 2.110 & 2.080 & 2.095 & 1.43 & $n / a$ & 1.000 & $\mathrm{n} / \mathrm{a}$ \\
\hline S97T001241 & D & Samarium-ICP-ACid Dill. & $\mathrm{ug} / \mathrm{mL}$ & 98.60 & $<1.00 \mathrm{e}-01$ & 2.000 & $<2.00 \mathrm{e} 0$ & $\mathrm{n} / \mathrm{a}$ & $\mathrm{n} / \mathrm{a}$ & $\mathrm{n} / \mathrm{a}$ & 2.000 & $n / a$ \\
\hline S97T001241 & D & Stront ium-ICP-Acid Dil. & $\mathrm{ug} / \mathrm{mL}$ & 99.60 & $<1.00 e^{-02}$ & $<2.00 \mathrm{e}-01$ & $<2.00 \mathrm{e}-1$ & $n / a$ & $\mathrm{n} / \mathrm{a}$ & $\mathrm{n} / \mathrm{a}$ & $2.00 e-01$ & $n / a$ \\
\hline S97T001241 & $D$ & Titanium-ICP-Acid oil. & $\mathrm{ug} / \mathrm{mL}$ & 99.20 & $<1.00 e-02$ & $<2.00 \mathrm{e}-01$ & $<2.00 e-1$ & $n / a$ & $\mathrm{n} / \mathrm{a}$ & $n / a$ & $2.00 \mathrm{e}-01$ & $n / a$ \\
\hline$\$ 977001241$ & $\bar{D}$ & Thallium-ICP-Acid Dil. & $\mathrm{ug} / \mathrm{mL}$ & 95.40 & $<2.00 \mathrm{e}-01$ & 4.000 & $<4.00 \mathrm{e} 0$ & $n / a$ & $\mathrm{n} / \mathrm{a}$ & $\mathrm{n} / \mathrm{a}$ & 4.000 & $n / a$ \\
\hline \$97T001241 & 0 & Uranium-ICP-ACId Dil. & $\mathrm{ug} / \mathrm{mL}$ & 97.00 & $<5.00 e-01$ & 10.00 & $<1.00 \mathrm{e} 1$ & $\mathrm{n} / \mathrm{a}$ & $n / a$ & $n / a$ & 10.00 & $n / a$ \\
\hline S97T001241 & D & Vanadium-ICP-Acid Dil. & $\mathrm{ug} / \mathrm{mL}$ & 101.0 & $<5.00 \mathrm{e}-02$ & 1.000 & $<1,00 \mathrm{e}^{0}$ & $\mathrm{n} / \mathrm{a}$ & $n / a$ & $\mathrm{n} / \mathrm{a}$ & 1.000 & $n / a$ \\
\hline S97T001241 & D & Zinc-ICP-ACid Dil. & $\mathrm{ug} / \mathrm{mL}$ & 97.20 & $<1.00 \mathrm{e}-02$ & $<2.00 e-01$ & $<2.00 \mathrm{e}-1$ & $n / a$ & $\mathrm{n} / \mathrm{a}$ & $n / a$ & $2.00 \mathrm{e}-01$ & $n / a$ \\
\hline S971001241 & $D$ & Zirconi Um-ICP-ACid Dil & $\mathrm{ug} / \mathrm{mL}$ & 99.40 & $<1.00 \mathrm{e}-02$ & $<2.00 \mathrm{e}-01$ & $<2.00 e-1$ & $n / a$ & $\mathrm{n} / \mathrm{a}$ & $\mathrm{n} / \mathrm{a}$ & $2.00 \mathrm{e}-01$ & $\mathrm{n} / \mathrm{a}$ \\
\hline S97T001241 & & Fluoride-IC-Dionex $4000 / 4500$ & $\mathrm{ug} / \mathrm{mL}$ & 104.6 & $<1.20 \mathrm{e}-02$ & 13.33 & $<1.33 \mathrm{e}$ & $n / a$ & $n / a$ & $n / a$ & 13.33 & $\mathrm{n} / \mathrm{a}$ \\
\hline \$97T001241 & & Chloride-IC-Dionex $4000 / 4500$ & $\mathrm{ug} / \mathrm{mL}$ & 100.5 & $<1.70 \mathrm{e}-02$ & 34.78 & 37.30 & 36.04 & 6.93 & $n / a$ & 18.89 & $\mathrm{n} / \mathrm{a}$ \\
\hline \$97T001241 & & Nitrite-IC - Dionex $4000 / 4500$ & $\mathrm{ug} / \mathrm{mL}$ & 100.2 & $<1.08 \mathrm{e}-01$ & $<1.20 \mathrm{e}+02$ & $<1.20 \mathrm{e} 2$ & $\mathrm{n} / \mathrm{a}$ & $n / a$ & $n / a$ & 120.0 & $\mathrm{n} / \mathrm{a}$ \\
\hline S97T001241 & & Nitrate by IC-D ionex $4000 / 4500$ & $\mathrm{ug} / \mathrm{mL}$ & 104.2 & $1.85 e-01$ & $<1.54 \mathrm{e}+02$ & 208.0 & $\mathrm{n} / \mathrm{a}$ & $\mathrm{n} / \mathrm{a}$ & $n / a$ & 154.4 & $\mathrm{n} / \mathrm{a}$ \\
\hline S97T001241 & & Phosphate-IC-Dionex $4000 / 4500$ & $\mathrm{ug} / \mathrm{mL}$ & 105.7 & $<1.20 \mathrm{e}-01$ & $<1.3 \overline{3 e+02}$ & $<1.33 \mathrm{e} 2$ & $n / a$ & $\mathrm{n} / \mathrm{a}$ & $\mathrm{n} / \mathrm{a}$ & 133.3 & $n / a$ \\
\hline S97T001241 & & Sulfate by IC-Dionex $4000 / 4500$ & $\mathrm{ug} / \mathrm{mL}$ & 106.8 & $<1.38 \mathrm{e}-01$ & $2.37 \mathrm{e}+02$ & 287.0 & 261.9 & 19.1 & $\mathrm{n} / \mathrm{a}$ & 153.3 & $n / a$ \\
\hline \$97T001241 & & Oxalate-IC-Dionex $4000 / 450$ & $\mathrm{ug} / \mathrm{mL}$ & 103.4 & $<1.05 e-01$ & $<1.17 \mathrm{e}+02$ & $<1.17 \mathrm{e} 2$ & $\mathrm{n} / \mathrm{a}$ & $\mathrm{n} / \mathrm{a}$ & $n / a$ & 116.7 & $\mathrm{n} / \mathrm{a}$ \\
\hline
\end{tabular}




\begin{tabular}{|c|c|c|c|c|c|c|c|c|c|c|c|c|}
\hline$E / U$ & $\varepsilon 0+280^{\circ} 1$ & $90^{\circ} 66$ & $5 L^{\circ} Z$ & $80+2259$ & $\varepsilon 0+265^{\circ} 9$ & $80+257^{\circ} 9$ & $10-240.1\rangle$ & $\varepsilon 6^{\circ} 76$ & $6 / 6 n$ & $0057 / 0007$ xaúl $01-21-278 j e \times 0$ & M & $79 \varepsilon 1001 \angle 6 \mathrm{~S}$ \\
\hline$e / \mathrm{u}$ & $E 0+\theta z \eta^{*}$ & 25.96 & $\mathrm{e} / \mathrm{u}$ & $e / 4$ & $\varepsilon^{2} \sigma \Sigma^{\circ} \mid>$ & $50+2 \overline{27} \cdot 1\rangle$ & $10-28 \varepsilon_{1} 1>$ & $70^{\circ} \varepsilon 6$ & $6 / 6 n$ & 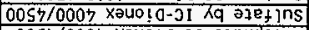 & M. & $79 \varepsilon 1001 \angle O S$ \\
\hline $\mathrm{E} / \mathrm{U}$ & $20+a 7 \bar{C}^{\circ} \mathrm{l}$ & $\mathrm{C}^{*} s \mathrm{ll}$ & $62 \% 9$ & $70+2 \varepsilon l \cdot 6$ & $70+2776$ & $70+228.8$ & $10-20 \overline{1} 1>$ & 06.86 & $6 / 6 n$ & 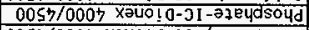 & $M$ & 7921001265 \\
\hline$e / 4$ & $\varepsilon 0+\partial \varepsilon^{\circ} 1$ & 9.921 & 26.7 & $50+0 \angle 8^{\circ} \varepsilon$ & $50+2 \angle L^{\circ} \varepsilon$ & $50+296^{\circ} \mathrm{E}$ & $10-\partial 6 \varepsilon \cdot 1>$ & $\angle Z^{\prime} \varepsilon 6$ & $6 / 6 n$ & 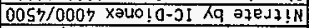 & $M$ & $49 \varepsilon 100126 \mathrm{~S}$ \\
\hline E/U & $\varepsilon 0+211 \cdot 1$ & $5 \xi^{\circ} 86$ & $\mathrm{e} / \mathrm{u}$ & $\mathrm{e} / \mathrm{u}$ & $\varepsilon^{0} 60^{\circ} \mid>$ & $\varepsilon 0+2 h l^{\prime} \downarrow>$ & $10-925, z$ & $0 E \cdot 76$ & $6 / 6 n$ & 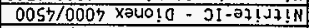 & $M$ & $79 \varepsilon 100126 \mathrm{~S}$ \\
\hline $\mathrm{E} / \mathrm{U}$ & $2 \cdot 52 l$ & $02 \div 96$ & e/u & E/U & $2 \ni l L l>$ & $20+\partial S L \cdot 1>$ & $20-20\langle 1\rangle$ & $70^{\circ} \varepsilon \sigma$ & $676 n$ & 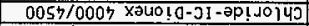 & M & $79 \varepsilon 1001265$ \\
\hline $\mathrm{E} / \mathrm{U}$ & $\angle E Z L$ & 0.801 & $80^{\circ} 6$ & $50+265^{\circ} 8$ & $\varepsilon 0+286^{\circ} 8$ & $\varepsilon 0+20 z^{\circ} 8$ & $50-905 \cdot 1>$ & 7.211 & 6/6n & $0057 / 0007$ xauolo-21-әp!jon 1 & Mi & $7981001 \angle 6 \mathrm{~S}$ \\
\hline 0 & 0.702 & $0 E^{\circ} \angle 6$ & $e / 4$ & $\mathrm{E} / \mathrm{u}$ & 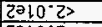 & $20+270^{\circ} Z>$ & $20-200^{\circ} 1>$ & 00.86 & $6 / 6 n$ & uo!sny-dJI- un! & 1 & $\sum 9 \varepsilon 1001 \angle 6 \mathrm{~S}$ \\
\hline$>$ & 0.702 & 08.96 & $\mathrm{e} / \mathrm{u}$ & $8 / 4$ & $2310^{\circ} 5>$ & $20+270^{\circ} \mathrm{Zz}$ & $20-200.13$ & $2 \cdot 101$ & $6 / 6 n$ & Uo!snt-dJI- Ju!z & I & $\varepsilon 9 \varepsilon 1001 \angle 6 \mathrm{~S}$ \\
\hline 4 & $\mathrm{EO} 0+250^{\circ} \mathrm{l}$ & 0586 & e/u & $e / 0$ & $\varepsilon^{2} 00^{\circ} \mid>$ & $50+220^{\circ} 1>$ & $20-200^{\circ} \mathrm{S}$ & 0266 & $6 / 6 n$ & Uo!sny-dJ - un!peueA & iो & $\varepsilon 9 \varepsilon 1001 \angle 6 \mathrm{~S}$ \\
\hline 뚜 & $70+220 \%$ & $00^{\circ} 78$ & E/u & $e / 4$ & $7000^{\circ} \mid>$ & $70+a 20^{\circ} l>$ & $10-700^{\circ} \zeta>$ & $0 \Sigma \varepsilon \sigma$ & $6 / 6 n$ & uo!sny-dJi - แn!uejก & ग) & EgELOOLLSS \\
\hline$\infty$ & $60+280^{\circ} 7$ & $01^{\circ} 56$ & $\mathrm{e} / \mathrm{u}$ & $8 / 4$ & $\left.\varepsilon^{2} 10^{\circ}\right\rangle$ & $\left.50+280^{\circ}\right)$ & $\left.10-200^{\circ} \mathrm{C}\right\rangle$ & 7.201 & 6/रิก & Uo!snł-dJ! - - & ji & $\varepsilon 9 \varepsilon \mathrm{L} 100126 \mathrm{~S}$ \\
\hline us & $0^{\circ}+02$ & 02.96 & $e / u$ & $e / 4$ & रण $10^{\circ} 5$ & $50+270,53$ & $50-500.1>$ & 0726 & $6 / 6 n$ & wo!sn $y-d \partial 1$ - & 1 & $\varepsilon 9 \varepsilon L 00126 \mathrm{~S}$ \\
\hline तु & 0.702 & $0 . \mathrm{LOL}$ & $0 / u$ & E/u & $2310^{\circ} 28$ & $50+2+0.5>$ & $20.000^{\circ} 13$ & 0.101 & $6 / 6 n$ & 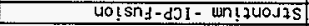 & d) & $\sum 9 \varepsilon 1001 \angle 6 \mathrm{~S}$ \\
\hline a & $\varepsilon 0+270^{\circ} 2$ & 0.701 & e/u & $e / u$ & $\varepsilon 10^{\circ} \mathrm{C}$ & $50+270^{\circ} \mathrm{C}>$ & $10-200^{\circ} 1>$ & $0.80 \mathrm{~L}$ & $6 / 6 n$ & wo!sn $y-d 31-$ win! Jeures & 1 & $\varepsilon 9 \varepsilon 1001 \angle 6 \mathrm{~S}$ \\
\hline $\bar{\Omega}$ & $\varepsilon 0+220^{\circ} l$ & 06.76 & 8.02 & $\varepsilon 0+286^{\circ} l$ & $\varepsilon 0+0 L L L$ & $50+28 l^{-2}$ & $20-200^{*} 5>$ & $09^{\circ} 96$ & $6 / 6 n$ & Uo!sny-d3I- 400!]!s & 1 & E9ELOOLLS \\
\hline$\frac{1}{3}$ & $20+\partial 22 \cdot l$ & 06.88 & e/u & $e / 4$ & $\varepsilon^{2} 02^{\circ} l>$ & $\varepsilon 0+2 Z z \mid>$ & $20-200^{\circ} 9>$ & $0 e^{-76}$ & $6 / 6 n$ & 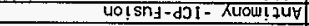 & I & घ9E1001L6S \\
\hline$\sum$ & $50+270^{\circ} z$ & 02.26 & e/u & $\mathrm{E} / \mathrm{U}$ & $\varepsilon^{2} 10^{\circ} Z>$ & $\left.\varepsilon 0+270_{0} 5\right\rangle$ & $10-500^{*} 1>$ & $09^{-9} 96$ & $6 / 6 n$ & Uo!sn=-dJI- In+ins & d & E9L1001L6S \\
\hline 5 & $20+070^{\circ} \mathrm{C}$ & $0 . \varepsilon 0 !$ & $E / 4$ & $\mathrm{e} / \mathrm{u}$ & $\varepsilon^{2} L 0^{\circ} Z^{\prime}$ & $\left.\varepsilon 0+270_{0} 5\right\rangle$ & $10-200^{\circ} 1>$ & $7.10 \mathrm{l}$ & $5 / 6 n$ & Uo! $s n_{y}-d \partial I-$ peat & if & E9ELOOLOS \\
\hline 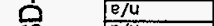 & $20+280^{\circ} 7$ & $05: 66$ & 9.91 & $70+2 \angle 6^{\circ} \varepsilon$ & $70+20 \varepsilon^{\circ} 7$ & $70+279^{\circ} \varepsilon$ & $10-000^{\circ} 5>$ & $02^{\circ} 66$ & $6 / 6 n$ & uo!snz-dJi - snjoydsoyd & 寸 & E9ELOOOLOS \\
\hline $\bar{\infty}$ & $\varepsilon 0+270^{\circ} \mathrm{C}$ & 0.201 & $8 / 4$ & $\mathrm{e} / \mathrm{u}$ & $\sum \geqslant 10^{\circ} 2>$ & $20+270^{\circ} 5>$ & $10-900.1>$ & $g^{\circ} \mathrm{gol}$ & $6 / 6 n$ & 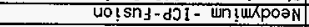 & 1) & E9ELOOLLOS \\
\hline$i$ & $50+240^{\circ} 2$ & $0 . \angle 91$ & $70-7$ & $50+22 L^{\circ} \mathrm{Z}$ & $50+282 \cdot 2$ & $50+2 \angle 9^{\circ} 2$ & $10-200^{\circ} \mid>$ & 9.001 & $6 / 6 n$ & Uo!sny-dJI- un!pos & ] & $\varepsilon 9 \varepsilon 1001 \angle 6 \mathrm{~S}$ \\
\hline$z$ & $\varepsilon 0+220 \cdot l$ & $01 \cdot 26$ & $E / U$ & $\mathrm{e} / \mathrm{u}$ & $\varepsilon 000^{\circ} 1>$ & $50+220^{\circ} 1>$ & $20-200^{\circ} 52$ & $200 l$ & $6 / 6 n$ & 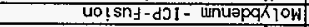 & I & 59ELOOLLOS \\
\hline$I$ & 0.702 & 0286 & $\mathrm{E} / \mathrm{u}$ & e/u & $2010^{\circ} 2>$ & $20+970^{\circ} 57$ & $50-900.1>$ & $900 \mathrm{~L}$ & $6 / 6 n$ & 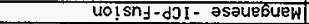 & 与 & S9ELO001L6S \\
\hline e/u & $\varepsilon 0+270^{\circ} z$ & $09^{\prime 26}$ & $e / \mathrm{U}$ & $\mathrm{e} / \mathrm{u}$ & Ealo $2>$ & $20+270_{0} 5>$ & $10-200-1\rangle$ & $07^{\circ} \angle 6$ & $6 / 6 n$ & uoisny-dJI- un! sausew & 5 & E9ELODLLOS \\
\hline $\mathrm{e} / \mathrm{u}$ & $\varepsilon 0+220^{\circ} l$ & 0.201 & $e / \mathrm{u}$ & e/u & $\Sigma 200^{\circ} \mid>$ & $50+220^{\circ} \mid>$ & $20-200^{2} \mathrm{~S}$ & $\rightarrow 101$ & $6 / 6 n$ & 4o!̣snd-dJI - wnueyzue? & 1 & S9ELOOLL6S \\
\hline $\mathrm{e} / \mathrm{u}$ & $50+220^{*} l$ & $0 \varepsilon^{\circ} 96$ & E/4 & $\mathrm{e} / \mathrm{u}$ & $\sum \ni 00^{\circ} \mid>$ & $20+20^{\circ} \mid>$ & $50-900.23$ & $02^{\circ} 66$ & $6 / 6 n$ & 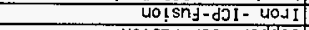 & 1 & E9ELOOOLOS \\
\hline $\mathrm{e} / \mathrm{u}$ & 0.702 & 0.501 & $e / 4$ & $e / u$ & $2010^{\circ} 27$ & $50+970_{0} \overline{5}$ & $20-200.1>$ & $8^{\circ} \mathrm{EOL}$ & $6 / 6 n$ & Uo!sny-dJI - JəddoJ & 与 & E9EL1001L6S \\
\hline $\mathrm{e/U}$ & 0.702 & $05^{\circ} \angle 6$ & $00^{\circ} 0$ & $50+0621$ & $20+26 L^{\prime} l$ & $80+2621$ & $20-200^{\circ} 12$ & $7.00 \mathrm{l}$ & $6 / 6 n$ & 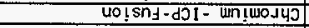 & d. & S9E1001L6S \\
\hline$e / u$ & 0.807 & $01 \div 26$ & $\mathrm{e} / \mathrm{u}$ & $\mathrm{e} / \mathrm{u}$ & $2^{2} 10^{\circ} \not>$ & $20+280^{\circ}+7>$ & $50-900,8>$ & 08.66 & $6 / 6 n$ & Uo!sny-dJI - I1eqoj & I & E9clooLL6s \\
\hline$e / 0$ & $80+270.2$ & $0^{\circ} 101$ & $\mathrm{E} / \mathrm{u}$ & $8 / \mathrm{U}$ & $\varepsilon^{2} 10^{\circ} z$ & $80+270.52$ & $10-200^{\circ} 1>$ & $2.00 \mathrm{l}$ & $6 / 6 n$ & Lo!snd-dJi- un! Jaj & ] & ع9El001L6S \\
\hline e/u & 0.201 & $05^{\circ} 86$ & $\mathrm{e} / \mathrm{u}$ & e/u & $2^{2} 00^{\circ} 1>$ & $20+950^{\circ} \mid>$ & $20-200^{-} \mathrm{s}$ & 0.101 & $6 / 6 n$ & uo!sny-dJI - unn!upej & ]) & E9ELOOLLOS \\
\hline $\mathrm{e} / \mathrm{u}$ & $\varepsilon 0+2+0^{\circ} z$ & $02 \div 26$ & $e / \mathrm{u}$ & E/u & $5010^{\circ} 2>$ & $\varepsilon 0+2700 \%$ & $10-200 \cdot 13$ & $08^{\circ} \angle 6$ & $6 / 6 n$ & uo!sng-dji - unis|ej & I & E951001L6S \\
\hline e/u & $\varepsilon 0^{2}+2 y 0^{-} Z$ & 0.101 & $\mathrm{e} / \mathrm{u}$ & $e / u$ & $\varepsilon^{\circ} 10^{\circ} 2>$ & $\varepsilon 0+270-2>$ & $10-200^{\circ} \mid>$ & $9 \cdot 201$ & $6 / 6 n$ & $40 ! s n y-d \partial I-47 n$ Is!g & d & E9ElOOOLOS \\
\hline E/u & 0.201 & $08^{\circ} 66$ & $e / \mathrm{u}$ & e/u & $2000^{\circ} 1>$ & $50+250.13$ & $20-200.2>$ & $8^{\circ} \mathrm{gOl}$ & $5 / 6 n$ & uo!snf-dəI - แn!l) Rdəg & If & E9ELO001/6S \\
\hline e/u & $50+220^{\circ} \mathrm{I}$ & $0^{\circ} \mathrm{ZOl}$ & $\mathrm{e} / \mathrm{u}$ & $\mathrm{e} / \mathrm{u}$ & $\sum 200^{\circ} \mid 1$ & $80+320^{\circ} 12$ & $20-200.5$ & $2 \div 201$ & $6 / 6 n$ & wo!sng-d3I - wn! Jeg & J & 5951001265 \\
\hline eju & $50+220^{\circ} i$ & $09^{\circ} 56$ & $\mathrm{e} / \mathrm{u}$ & $\mathrm{E} / \mathrm{u}$ & $\varepsilon^{2} 00^{\circ} 1 \mathrm{~s}$ & $\varepsilon 0+a_{20} l>$ & $20-200.5$ & 08.66 & $6 / 6 n$ & Uo!sny-dJI - UoJog: & J & c9c100!L6s \\
\hline efu & $\varepsilon 0+270^{\circ} \mathrm{Z}$ & 0.101 & $\mathrm{e} / \mathrm{u}$ & $\bar{e} / \mathrm{u}$ & $\varepsilon 210^{\circ} \mathrm{z}$ & $\varepsilon 0+270^{\circ} Z>$ & $10-200 \cdot 1>$ & $8^{\prime} \mathrm{gOL}$ & $6 / 6 n$ & Uo!sng-dJ!- ग!uəs.ly & J & $\sum 9 \sum L 00 \perp \angle 6 S$ \\
\hline e/U & $50+220^{\circ} \mathrm{l}$ & $0^{\circ} \mathrm{2Ol}$ & $66^{\circ} \mathrm{l}$ & $\Sigma 0+\partial \xi 0^{\circ} L$ & $\mathrm{CO}+20 \mathrm{~L}^{\circ} \mathrm{L}$ & $\varepsilon 0+296^{\circ} 9$ & $20-200^{\circ} \varsigma>$ & 8.001 & $6 / 5 n$ & ưo!snj-d & I) & E9ElO0OLLS \\
\hline $8 / 4$ & 0.702 & $06^{\circ}: 21$ & $\mathrm{e} / \mathrm{u}$ & e/u & $2010^{\circ} 27$ & $20+240^{\circ} 22$ & $20-200^{\circ} 1>$ & $9^{\circ} 701$. & $6 / 6 n$ & 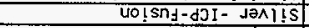 & \pm & E9EL001L6S \\
\hline \%dJ 7unos & $7 ! 6 ! 7720$ & $\%$ गेy Xds & $\%$ Odd & Fूejan & 2zeo!)ano & 7 insoy & त्रueig & $\%$ p.sepueis & 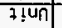 & क्रा & $\# \forall$ & \#ंjowes \\
\hline
\end{tabular}

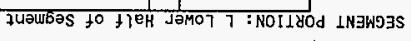

$\iota:$ : 1NقW

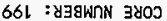

$10 l-18$

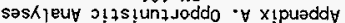




\begin{tabular}{|c|c|c|c|c|}
\hline \multirow{2}{*}{$\begin{array}{l}\text { To } \\
\text { Distribution }\end{array}$} & \multirow{2}{*}{\multicolumn{2}{|c|}{ From }} & \multicolumn{2}{|c|}{ Page 1 of 1} \\
\hline & & & \multicolumn{2}{|c|}{ Date: $09 / 23 / 97$} \\
\hline \multirow{2}{*}{\multicolumn{3}{|c|}{$\begin{array}{l}\text { Project Title/wark Order } \\
\text { HNF-SD-WM-DP-258, Rev. } 0 \text { "Tank 241-BY-101, Cores } 189 \text { and } 199 \\
\text { Analytical Results for the Final Report" }\end{array}$}} & \multicolumn{2}{|c|}{ EDT N0.: EDT-620423 } \\
\hline & & & \multicolumn{2}{|c|}{ ECN N0.: N/A } \\
\hline & MSIN & $\begin{array}{l}\text { Text With } \\
\text { a1] Attach }\end{array}$ & $\begin{array}{l}\text { EDT/ECN } \\
\text { ONLY }\end{array}$ & \\
\hline
\end{tabular}

DE\&S Hanford, Inc.

J. E. Meacham

S7-14 X

U.S. Department of Energy, RL

C. A. Babel

S7-54 X

Lockheed Mart in Hanford Corp.

J. N. AppeI

G3-21

K. G. Carothers

R2-11

J. Jo

T. J. Kelley

R2-12

S7-21

M. J. Kupfer

H5-49

J. B. Schaffer

R2-12

L. A. Stauffer

$\mathrm{R} 2-11$

J. A. Voogd

H5-03

A. E. Young

$\mathrm{R} 1-10$

$X$
$X$
$X$
$X$
$X$
$X$
$X$

Lockheed Martin Services, Inc.

Central Files

A3-88 $\quad 1$

Los Alamos Technical Associates

M. T. Ellsworth

T6-06

$X^{*}$

Pacific Northwest National Laboratory

S. J. Harris

$\mathrm{K} 7-22$

K9-08

$x$

K. L. Silvers

$56-31$

T6-06

T6-12

T6-14

T6-03

$x$

Waste Management of Hanford. Inc.

D. C. Hetzer

J. L. Nuzum

K. L. Powe 11

C. M. Seidel

$\mathrm{H} 5-27$

$x$

BGN Eurisys

Defense Nuclear Federal Safety Board

Rich Tontodonato

625 Indiana Ave. N. W.

$\mathrm{X}$

Washington D.C. 20004

* Needs only releasing paperwork, not a copy of the released document. 